\title{
THE GENUS GERANIUM L. (GERANIACEAE) IN NORTH AMERICA. II. PERENNIAL SPECIES
}

\author{
by \\ CARLOS AEDO \\ Real Jardín Botánico, CSIC. Plaza de Murillo, 2. E-28014 Madrid \\ (aedo@ma-rjb.csic.es)
}

\begin{abstract}
Resumen
AEDo, C. (2001). El género Geranium L. (Geraniaceae) en Norteamérica. II. Especies perennes. Anales Jard. Bot. Madrid 59(1): 3-65 (en ingless).

Se revisan las especies perennes de Geranium de Norteamérica al N de México. Se aceptan 19 especies de las cuales 17 pertenecen al subg. Geranium y dos al subg. Robertium. Geranium erianthum, G. maculatum, G. oreganum, G. sylvaticum, G. caespitosum, G. californicum, G. lentum, G. wislizenii, G. richardsonii, $G$. viscosissimum son las especies autóctonas, y G. pratense, G. sanguineum, G. potentilloides, G. sibiricum, G. thunbergii, G. retrorsum, G. solanderi, G. pyrenaicum y G. palmatum, las introducidas tanto desde Europa como desde Asia u Oceanía. Geranium eremophilum, G. fremontii, G. parryi, G. marginale y G. cowenii, a veces aceptados en la literatura reciente, son considerados como sinónimos de G. caespitosum. Del mismo modo, se sinonimiza $G$. strigosum y $G$. attenuilobum a $G$. viscosissimum y G. concinnum a $G$. californicum. Se revisa la nomenclatura de todas las especies y se designan diecisiete lectótipos. Se incluyen descripciones completas, una clave, mapas de distribución e ilustraciones.
\end{abstract}

Palabras clave: Geranium, Geraniaceae, taxonomía, tipificaciones, Norteamérica.

\begin{abstract}
AEDO, C. (2001). The genus Geranium L. (Geraniaceae) in North America. II. Perennial species. Anales Jard. Bot. Madrid 59(1): 3-65.

Perennial species of Geranium from North America north of Mexico are revised. Nineteen species are accepted, of which seventeen belong in subg. Geranium and two belong in subg. Robertium. Geranium erianthum, G. maculatum, G. oreganum, G. sylvaticum, G. caespitosum, $G$. californicum, $G$. lentum, $G$. wislizenii, $G$. richardsonii, $G$. viscosissimum are native, whereas G. pratense, G. sanguineum, G. potentilloides, G. sibiricum, G. thunbergii, G. retrorsum, G. solanderi, G. pyrenaicum and G. palmatum were introduced from Europe, Asia, and Oceania. Geranium eremophilum, G. fremontii, G. parryi, G. marginale and G. cowenii, sometimes accepted in current literature, are considered to be synonyms of $G$. caespitosum. Similarly, $G$. strigosum and $G$. attenuilobum are synonymized to $G$. viscosissimum, and G. concinnum to $G$. californicum. Nomenclature for all species is reviewed, and seventeen lectotypes are designated. Descriptions, key, distribution maps, and illustrations are included.
\end{abstract}

Key words: Geranium, Geraniaceae, taxonomy, typification, North America. 


\section{INTRODUCTION}

The study of Geranium from North America has been divided in two parts for operative reasons. The first part, including annual (and biennial) species was published in AEDo (2000). The second part revising perennial species is presented here.

General information about North American Geranium was given in the introduction of AEDo (2000), and is not repeated. Additionally, the study by JONES \& JONES (1943) should be remarked. These authors revised perennial species from the United States and Canada. They considered eighteen species from which only one was introduced (G. pratense). Their treatment is more analytic, since they split $G$. caespitosum in six species, G. viscosissimum in three species, and $G$. californicum in two species.

Geranium comprises in the area of North America flora 30 species (both annuals and perennials), of which 23 belong in subg. Geranium and 7 belong in subg. Robertium. All native species (13) belong in subg. Geranium. Among the 17 introduced species, 10 belong in subg. Geranium and 7 belong in subg. Robertium. From these introduced species 13 have arisen from the Old World (Europe and neighbouring areas), 3 from Oceania, and 1 from East Asia.

Some remarks should be also made about the distribution of native perennial species. Two of them also occur in another continent: $G$. erianthum in Asia, $G$. sylvaticum in Europe. Among the remaining, two are widespread in North America, one is restricted to the Eastern half of the continent, and eight are restricted to the opposite half. Thus, the Rocky Mountains and western areas seem to be the richest in Geranium species. The number of endemic species is eight. Geranium caespitosum, G. carolinianum, and $G$. wislizenii have their Southern limit in Mexico. Finally, six species do not occur in Canada: G. caespitosum, G. californicum, G. lentum, G. oreganum, G. texanum, and $G$. wislizenii.

\section{MATERIALS AND METHODS}

This revision is based on more than 2000 herbarium specimens from the following herbaria: ARIZ, BM, CAN, CM, COLO, DAO, F, GH, ILL, JEPS, K, LE, MA, MEXU, MICH, MIN, MO, MSC, NEB, NMC, NY, OKL, P, PH, SD, TEX, UC, UNM, US, V, VT, W, and WTU. Furthermore, microfiches, photographs, and other material have been examined from $B M$ and LINN. Curators from ALTA, ACAD. BRY, MOAR, UAT, and UBC, kindly answered our petition, but they did not find any of the requested specimens in their herbaria.

The ten native species have been rather thoroughly studied in some aspects such as morphology, synonymy, and distribution. In those native species, a search for micromorphological characters has been carried out using scanning electron microscopy (SEM). Samples were glued to aluminium stubs, coated with 40-50 nm gold, and examined with a JEOL-TSM T330A scanning electron microscope at $15 \mathrm{kV}$.

Short glandular hairs ( $<40 \mu \mathrm{m}$ long), usually constituted by two cells (although they sometimes have a bicellular foot, Fig. 1f), are present in all species here studied but they are not considered in descriptions since they are only evident at high magnification.

For introduced species only synonyms described from North America are indicated, while a comprehensive synonymy is provided for natives ones.

The area covered in this paper includes North America north of Mexico plus Greenland, since this study has been carried out for the Flora of North America project, which includes the above mentioned area. For eight native Geranium, maps cover the total range of the each species. However, for $G$. erianthum and G. sylvaticum maps only include American localities. For the introduced species maps are restricted to its North American area. 

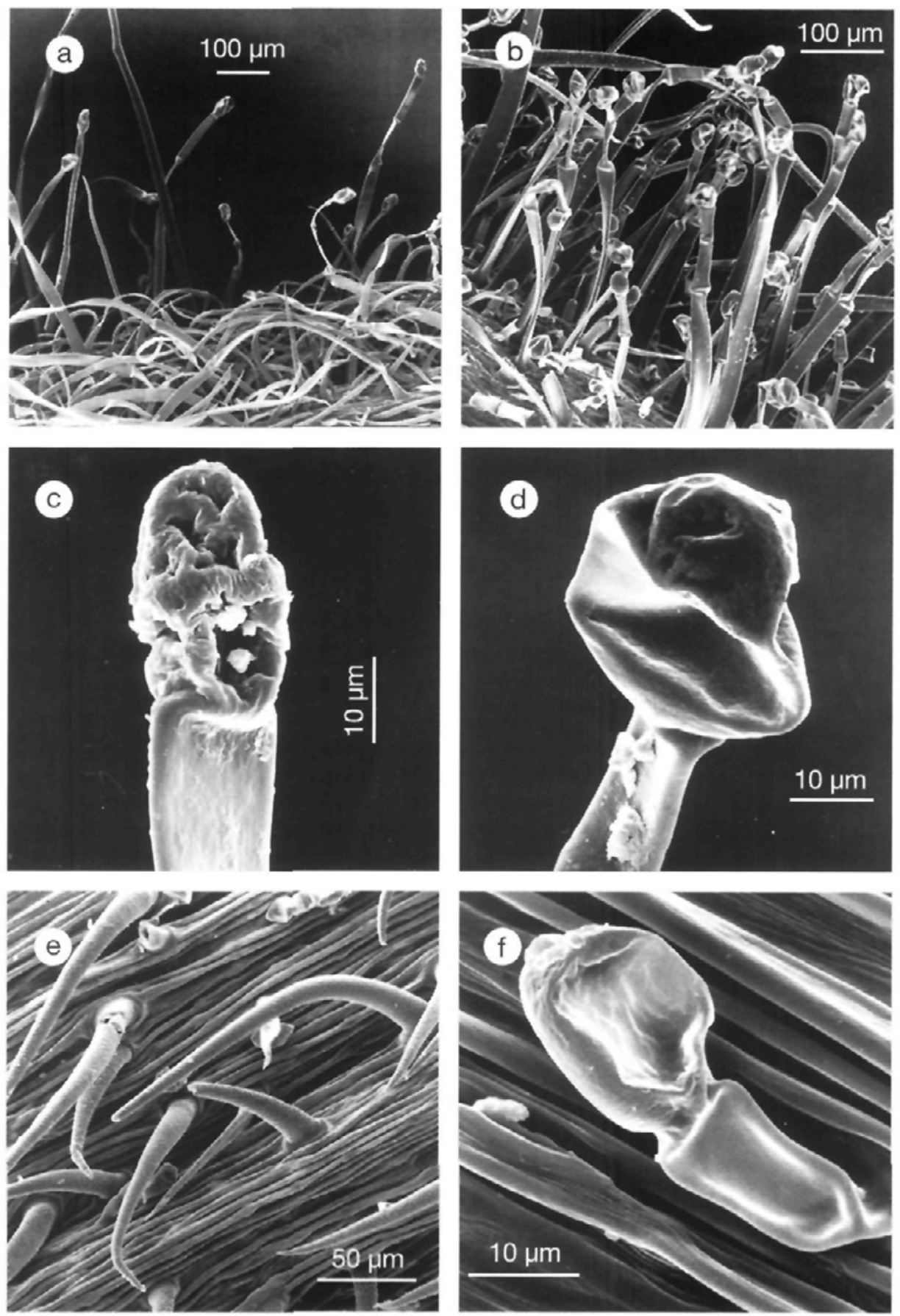

Fig. 1.-SEM photomicrographs showing indumentum of some perennial Geranium native from North America: a, pedicel of G. erianthum [Calder 5411 (DAO)]; b, pedicel of G. viscosissimum [Peck 18320 (ORE)]; c, apex of a glandular hair on the sepal of G. maculatum [Ahles 82611 (VT)]; d, apex of a glandular hair on the pedicel of $G$. viscosissimum [Peck 18320 (ORE)]; e, pedicel of G. maculatum [Ahles 82611 (VT)]; f, small glandular hair on the pedicel of $G$. caespitosum [Spellenberg $6324(\mathrm{NMC})]$. 


\section{KEY TO THE SPECIES}

1. Cymules 1-flowered 2

1. Cymules 2-flowered 4

2. Petals more than $15 \mathrm{~mm}$ long 6. G. sanguineum

2. Petals $5-10 \mathrm{~mm}$ long 3

3. Leaves with middle segment obtriangular, 3 (-5)-lobed at the apex ...... 7. G. potentilloides

3. Leaves with middle segment rhombic, 7-9lobed in the distal half. 8. G. sibiricum

4. Mericarps without basal callus 5

4. Mericarps with basal callus

5. Petals 7-11 mm long 18. G. pyrenaicum

5. Petals $19-30 \mathrm{~mm}$ long

6. Mericarps reticulate 19. G. palmatum

6. Mericarps smooth G. $\times$ magnificum

7. Petals glabrous on the adaxial surface, ciliate at the base .8

7. Petals hairy on 1/3-3/4 of their adaxial surface 15

8. Petals $5-12 \mathrm{~mm}$ long; fruit $13-23 \mathrm{~mm}$ long $\ldots 9$ 8. Petals $12-21 \mathrm{~mm}$ long; fruit $27-52 \mathrm{~mm}$ long 11

9. Calyx with glandular hairs ... 9. G. thunbergii

9. Calyx without glandular hairs 10

10. Pedicels with retrorse, appressed, eglandular hairs; sepal mucro 0.7-1 mm long, without bristhes 10. G. retrorsum

10. Pedicels with patent or reflexed (never appressed), eglandular hairs; sepal mucro 0.4-0.5 $\mathrm{mm}$ long ending in $1-3$ bristles $0.3-0.4 \mathrm{~mm}$ long

11. G. solanderi

11. Staminal filaments with eglandular hairs 1-3 $\mathrm{mm}$ long 1. G. erianthum

11. Staminal filaments with eglandular hairs up to $0.7 \mathrm{~mm}$ long 12

12. Pedicels with eglandular hairs, without glandular hairs 2. G. maculatum

12. Pedicels densely glandular-hairy 13

13. Nectarious glabrous 4. G. sylvaticum

13. Nectarious with a tuft of hairs at the top ..... 14

14. Fruit $34-36 \mathrm{~mm}$ long, downwardly inclined or reflexed when immature; staminal filaments with an expanded base 5. G. pratense

14. Fruit $35-42 \mathrm{~mm}$ long, erect when immature; staminal filaments lanceolate ..... 3. G. oreganum

15. Petals 5-9(-10.5) $\mathrm{mm}$ long; sepals $4.5-8 \mathrm{~mm}$ long 16

15. Petals $11-20 \mathrm{~mm}$ long; sepals $7-11 \mathrm{~mm}$ long 17

16. Nectaries glabrous; stigmatic remains $4-5 \mathrm{~mm}$ long

14. G. lentum

16. Nectaries dorsally hairy; stigmatic remains 1.5 $3 \mathrm{~mm}$ long 15. G. wislizenii
17. Nectarious dorsally lanate; petals usually white 16. G. richardsonii

17. Nectarious with a tuft of hairs at the top, dorsally glabrous; petals white or pink ............. 18

18. Ratio fruit length $/$ stigmatic remains length $=$ 6-8.2; basal leaves $7.1-16 \mathrm{~cm}$ wide

17. G. viscosissimum

18. Ratio fruit length $/$ stigmatic remains length $=$ 3.8-5.5; basal leaves $3-7.5 \mathrm{~cm}$ wide 19

19. Fruit $22-31(-33) \mathrm{mm}$ long; rostrum with narrowed apex 2-4 mm long; petals usually purplish; pedicels short $(1.8-12 \mathrm{~cm}) \ldots 12$. G. caespitosum

19. Fruit $32-42 \mathrm{~mm}$ long; rostrum with narrowed apex 5-6 mm long; petals usually white; pedicels long $(2.2-24 \mathrm{~cm})$ 13. G. californicum

\section{TAXONOMY}

\section{Geranium subg. Geranium}

Fruit of "seed-ejection-type," with seeds actively discharged by the explosive recoiling of the awn, which remains together with the mericarp attached to the columella; mericarps with basal callus or prong.

1. Geranium erianthum DC., Prodr. 1: 641 (1824) G. pratense var. erianthum (DC.) B. Boivin in Naturaliste Canad. 93: 1060 (1967) Ind. loc.: "in Kamtschatkâ et Amer. bor. et occidentali. Nelson. (v. s.)"

Typus: USA. Alaska, Newenham, Nelson s.n. (lectotype, here designated, G-DC micro!) [58 $\left.39^{\prime} \mathrm{N}, 162^{\circ} 10^{\prime} \mathrm{W}\right]$

= G. erianthum var. elatum Maxim., Prim. Fl. Amur.: 71 (1859)

G. elatum (Maxim.) R. Knuth in Engl., Pflanzenr. IV.129 (Heft 53): 113 (1912)

Ind. loc.: "Hab. In der Nadelwaldregion des untern Amur: bei Ssutschu, 19 Juni, und bei Kitsi, 20 Juni 1855 (flor.), in Laubgehölzen häufig"

Typus: Russia. Amur, Ssutschu, 19 Jun. 1855, Maximowicz s.n. (lectotype, designated by CHARKEVICZ, 1988: 142, LE!)

$=G$. eriostemon var. orientale Maxim. in Bull. Acad. Imp. Sci. Saint-Pétersbourg 26: 464 (1880)

G. gorbizense Aedo \& Muñoz Garm. in Kew Bull. 52(3): 725 (1997)

G. orientale (Maxim.) Freyn in Oesterr. Bot. Z. 52: 18 (1902) [syn. subst.], nom. illeg., non Mill. (1768) 
Ind. loc.: "Mandshuria: ad Schilkam inferiorem, 70 stadia infra Gorbiza, in laricetis siccioribus frequens, medio Junio fl., ad Amur superiorem, silvis frondosis lapidosis montanis ad Tolbusino et Tschernäjewa frequens, ad Amur inferiorem, lucis pratisque humidis prope Kitsi, frequens, ad Usuri superiorem prope Dsiabigo in montibus lapidosis, fine Maji fl. incip., similibus locis secus fl. LiFudin, et circa portum S-tae Olgae, med. Junio fl. c. fr. immat.; Japonia: Nippon, ad rupes secus rivulos in alpe Niko (Tschonoski), prov. Senano (Tanaka, fide Franchet)"

Typus: Russia. St Olga, 21 Jun. 1860, Maximowicz s.n. (lectotype, designated by Novoselova, in sched. 1998, LE!) [lectotype according to CHARKEVICZ (1988: 142) should be rejected, since it was collected in 1899]

$=$ G. erianthum f. leucanthum Takeda in Bot. Mag. (Tokyo) 24: 258 (1910)

Ind. loc.: "Hab. Yezo: in promontorio Tumoshiri prope Nemuro (H. Takeda! 12. VII. 1909); in herbosis Shunapaushi, prope oppid. Nemuro (H. Takeda! 5 et 10 VII. 1909)" Typus: Japan. in herbosis Shunapaushi, prope Nemuro, 5-10 Jul. 1909, Takeda s.n. (lectotype, here designated, BM!)

= G. erianthum f. albiflora Kom., F1. Kamtschatka 2: 295 (1929)

Ind. loc.: "Paratunka near Nikolaevskaya Bolsheretzka villages, 21 VII 1908, V. Komarov" [in Russian]

Typus: Russia. Paratunka near Nikolaevskaya Bolsheretzka villages, $21 \mathrm{Jul}$. 1908, Komarov s.n. (no original specimen located) [52 $\left.57^{\prime} \mathrm{N}, 158^{\circ} 14^{\prime} \mathrm{E}\right]$

$=$ G. erianthum f. alpestris Kom., $\mathrm{Fl}$. Kamtschatka 2: 295 (1929)

Ind. loc.: "1908, 1. 17 VIII Shapochka;2.23 VIII Ranges Krasny; 3. 13 IX, Chemushka.1909, 1. 1 IX Hills Krasheninnikova; VII 1909 Cape Lopatka; 31 VII 1908 Kluchevskaya, V. Komarov" [in Russian] Sintypus: Russia: Shapochka, 17 Aug. 1908, Komarov s.n.; Ranges Krasny, 23 Aug. 1908, Komarov s.n.; Chemushka, 13 Sep. 1908, Komarov s.n.; Krasheninnikova, 1 Nov. 1909, Komarov s.n. [50'17'00'N, $155^{\circ} 20^{\prime} 25^{\prime \prime} \mathrm{E}$; Cape Lopatka, Jul. 1909, Komarov s.n. [5052'00’'N 156 $\left.40^{\circ} 00^{\prime \prime} \mathrm{E}\right]$;
Kluchevskaya, 31 Jul. 1908, Komarov s.n. [56 $04^{\circ} 00^{\prime \prime} \mathrm{N} 160^{\circ} 38^{\prime} 00^{\prime \prime} \mathrm{E}$ ] (no original specimen located)

= G. erianthum f. communis Kom., F1. Kamtschatka 2: 294 (1929), nom. inval. (Code, art. 24.3)

$=$ G. erianthum var. angustifolium Miyabe \& Tatew. in Trans. Sapporo Nat. Hist. Soc. 14: 263 (1936)

Ind. loc.: "Hab. S. Saghalien. Distr. Shikka: The upper Ennai (M. Kawashima, VII. $28,1935)$ "

Typus: Russia. Sakhalin, Shikka, Ennai, 28 Jul. 1935, Kawashima s.n. (no original specimen located)

Herbs perennial, $10-50 \mathrm{~cm}$ tall. Rootstock 5-8 mm diam., horizontal, not tuberculate, not turnip-shaped. Stem erect, solitary, with retrorse, appressed, eglandular hairs 0.2-0.5 $\mathrm{mm}$ long. Basal leaves in a persistent rosette; lamina $3-10 \times 4.5-15 \mathrm{~cm}$, polygonal in outline, cordate, palmatifid (divided for 0.69 0.82 of its length), pilose, with appressed, eglandular hairs; segments 7, rhombic, $9-11 \mathrm{~mm}$ at the base, 20-34-lobed in distal half (ratio main-sinus length of the middle segment / middle-segment length $=0.18-0.32$ ); cauline leaves alternate (usually 1-2); petioles to 24 $\mathrm{cm}$ long, with retrorse, appressed, eglandular hairs 0.2-0.5 mm long; stipules 9-14 × 2-4.5 $\mathrm{mm}$, lanceolate, with eglandular hairs on abaxial surface and on the margin, glabrous adaxially. Inflorescence a dichasial cyme; cymules 2-flowered, solitary or in aggregates at the top of each branch; peduncles $0-3.7 \mathrm{~cm}$

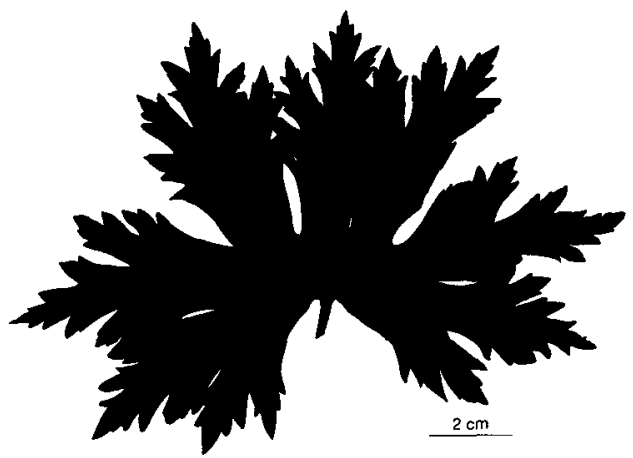

Fig. 2.-Silhouette of lamina of Geranium erianthum [Soule 533 (MO)] 
long, pilose, with retrorse, eglandular hairs 0.2-0.6 mm long, rarely with glandular hairs 1.1-1.9 mm long; bracteoles 2-3 $\times 1 \mathrm{~mm}$, linear-lanceolate, with eglandular and glandular hairs on abaxial surface and on the margin, glabrous adaxially; pedicels $0.4-1.8 \mathrm{~cm}$ long, with eglandular, patent to retrorse hairs 0.2 $0.6 \mathrm{~mm}$ long, and usually with glandular hairs 1.1-1.9 $\mathrm{mm}$ long; pedicel and peduncle together not overtopping the subtending leaf. Sepals 7-10 × 3-4 mm (ratio pedicel length / sepal length $=0.6-1.8$ ), smooth, not accrescent, 3-5-nerved, with mucro 1-2.5 mm long, with scarious margins $0.1-0.2 \mathrm{~mm}$ wide, with eglandular, antrorse, appressed hairs 0.2-0.5 $\mathrm{mm}$ long and glandular, patent hairs $0.9-2 \mathrm{~mm}$ long on the abaxial side, glabrous adaxially. Petals 15-20 $\times$ 9-12 mm (ratio petal length / petal width $=1.1-1.6$ ), erect-patent, entire, without claw, glabrous on the adaxial side, hairy on the base of abaxial side, ciliate on the basal margin, purplish. Stamens 10 , both whorls bearing anthers; filaments $9-11 \mathrm{~mm}$ long, not exserted, lanceolate with an abruptly narrowed apex, pilose on the abaxial side, ciliate on the proximal half, with hairs 1-3 $\mathrm{mm}$ long; anthers $2.5-3 \times 1 \mathrm{~mm}$. Nectaries glabrous. Gynoecium 9-10 mm long. Fruit 30-37 mm long, erect when immature; mericarps $3.5-4 \times 2-2.5 \mathrm{~mm}$, without a strand of fibres, smooth, without longitudinal rib, without basal beak, with a basal callus, with eglandular, \pm patent hairs 0.1-0.6 mm long, and glandular hairs 1-1.4 mm long, brownish; rostrum 25-29 mm long, with a narrowed apex 5$6 \mathrm{~mm}$ long, with \pm patent, eglandular hairs 0.1-0.2 mm long, and glandular 1-1.2 mm long; stigmatic remains $2 \mathrm{~mm}$ long (ratio fruit length / stigmatic remains length $=14-16$ ), with 5 glabrous lobes. Seeds $2-2.1 \times 1.7 \mathrm{~mm}$, smooth, brownish; hilum $1 / 4$ as long as the perimeter. Cotyledons entire. $2 n=28$.

Flowering June-August. Sandy grassy beach, meadows, bogs, and alpine slopes; 0 $1600 \mathrm{~m}$. China, Siberia, Subartic America \& Western Canada. Fig. 3.

Illustrations. Fig. 1a, 2, 5a, 8a, 30a. YEO (1992a: 90 fig. 26, pl. 13, 14).
As YEO (1992a: 88) pointed out, G. erianthum is quite similar to $G$. platyanthum Duthie [G. eriostemum Fisch. ex DC., nom. illeg., non Poir. (1812)], both presenting stamen filaments covered with long hairs. It, however, differs in the lower stems with eglandular not spreading hairs, and with more deeply divided and more acutely and profusely lobed and toothed leaves. Geranium platyanthum is endemic to East Asia where G. erianthum also occurs.

The specimens supporting the record of G. erianthum from Alberta (SCOGGAN 1978: 1044; PACKER 1983: 393) have not been studied.

\section{Representative specimens examined}

Canada. British Columbia: $3 \mathrm{mi}$ S of Ritchie Creek on Stewart-Watson Lake road, $56^{\circ} 27^{\prime} \mathrm{N}, 129^{\circ} 24^{\prime} \mathrm{W}, 2$ VII-1973, Beanish \& al. s.n. (DAO); 9-Mile Mountain, Hazelton, $54^{\circ} 35^{\prime} \mathrm{N}, 128^{\circ} 0^{\prime} \mathrm{W}, 5$-VIII-1940, McCabe 8160 (WTU); Chilkat Pass, 59 $41^{\prime}$ 'N, $136^{\circ} 33^{\prime} \mathrm{W}, 17$-VII1973, Douglas 6017 (DAO); East Ravine of Birch Mountain, Teresa Island, Atlin Lake, $59^{\circ} 25^{\prime} \mathrm{N}, 133^{\circ} 45^{\prime} \mathrm{W}, 16-$ VIII-1975, Buttrick 759 (DAO); Evelyn, near Smithers, $54^{\circ} 53^{\prime} \mathrm{N}, 127^{\circ} 16^{\circ} \mathrm{W}, 14-\mathrm{VIII}-1939$, Groh 585 (DAO); Fraser, mile 27 on Yukon-White Pass Railway, 59 $37^{\prime} \mathrm{N}$, 135 59'W, 11-VII-1949. Gillet \& Mitchell 3667 (DAO); Haines road, mi $83,59^{\circ} 50^{\prime} \mathrm{N}, 137^{\circ} 0^{\prime} \mathrm{W}, 6-\mathrm{VII}-1956$, Taylor \& al. 926 (DAO); Hazelton, $55^{\circ} 15^{\prime} \mathrm{N}, 127^{\circ} 40^{\prime} \mathrm{W}$, 8-VI-1949. Emmerson 33 (DAO); Hudson Bay, above Sylvan Mine, $9 \mathrm{ml}$ W Smithers, $59^{\circ} 50^{\prime} \mathrm{N}, 137^{\circ} 0^{\prime} \mathrm{W}, 13-$ VII-1954, Calder \& al. 12946 (DAO); mi 38 along the Nilkitwa Forest, $55^{\circ} 14^{\prime}$ N, $126^{\circ} 38^{\prime}$ W, 22-VII-1974, Krajina \& al. 1974 (DAO); mile 63.5 Haines Road, 59 39'N, 136³0'W, 9-VII-1975, Douglass 8608 (DAO); Moosehorn Lake. $58^{\circ} 10^{\prime} \mathrm{N}, 132^{\circ} 7^{\prime} \mathrm{W}, 28$-VII-1960, Pilfrey 61 (DAO); Pete Lake, 57 $56^{\prime} \mathrm{N}, 133^{\circ} 56^{\prime} \mathrm{W}, 20$-VIII- 1960. Pilfrey 110 (DAO); Red Rose Tungsten mine about $9 \mathrm{mi}$ E of Skeena Crossing, $55^{\circ} 5^{\prime} \mathrm{N}, 127^{\circ} 48^{\prime} \mathrm{W}, 22-\mathrm{VII}-1954$,

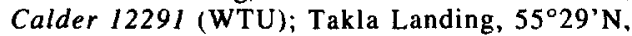
125'58'W, 26-VII-1940, Mc Cabe 8049 (WTU). YukoN: Alsek River, $2.5 \mathrm{~km} \mathrm{~N}$ of B.C. border, $60^{\circ} 1$ ' $\mathrm{N}$, 137 51'W, 27-VII-1975, Douglas 8951 (DAO); Bulion Creek, $60^{\circ} 58^{\prime} \mathrm{N}, 138^{\circ} 36^{\prime} \mathrm{W}, 24-\mathrm{VI}-1970$, Pearson \& Porsild 5996 (DAO); Cassiar Mountains, near the Smart River Creek, 605'N, $131^{\circ} 30^{\prime} \mathrm{W}, 27-$ VII-1952, Poole s.n. (DAO); Haines road km 86, Klehini River valley, $59^{\circ} 33^{\prime} \mathrm{N}, 136^{\circ} 31^{\prime} \mathrm{W}, 25-\mathrm{VI}-1979$, Douglas 11016 (WTU); Profile mtn., Kluane National Park, 60 $37^{\prime} \mathrm{N}$, 137 55'W, 24-VII-1973, Douglas 6412 (WTU): west side of Alsek river, $\mathrm{N}$ of Lowell Glacier, $60^{\circ} 17^{\prime} \mathrm{N}$. $138^{\circ} 0^{\prime}$ W, 28-VII-1970, Pearson \& Porsild 5994 (DAO).

USA. ALASKA: Afognak Quad, Afognak I., vicinity of Dig Afognak 1999 archeology site, $58^{\circ} 0^{\prime} \mathrm{N}, 152^{\circ} 46^{\prime} \mathrm{W}$, 14-VII-1999, Jones s.n. (MA); Anchorage, Chugash Mountains, $61^{\circ} 25^{\prime} \mathrm{N}, 149^{\circ} 23^{\prime} \mathrm{W}, 14-\mathrm{VII}-1947$, Dutilly \& al. 21209 (DAO); Chignik Bay, 56 $23^{\prime} \mathrm{N}, 158^{\circ} 13^{\prime} \mathrm{W}, 27$ VII-1931, Norberg 2 (WTU); Disenchatment Bay, in the 
vicinity of Yakutat Bay, $59^{\circ} 43^{\prime} \mathrm{N}, 139^{\circ} 50^{\prime} \mathrm{W}, 10$-VIII1892, Funston 100 (MO); East of Maclaren river, mi 61 Denali Highway, $62^{\circ} 50^{\prime} \mathrm{N}, 147^{\circ} 7^{\prime} \mathrm{W}, 27-\mathrm{VII}-1960$, Dempster 1335 (UC); Juneau, $58^{\circ} 18^{\prime} \mathrm{N}, 134^{\circ} 25^{\prime} \mathrm{W},-\mathrm{VI}$,

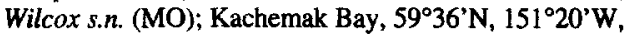
18-VI-1902, Gorman 1559 (WTU); kagway-HoonahAngoon Co., Windham Bay, $57^{\circ} 34^{\prime} \mathrm{N}, 133^{\circ} 27^{\prime} \mathrm{W}, 28-$ VII-1905, Culberton \& al. 4945 (MO); Karluk, 57³4'N, $154^{\circ} 27^{\prime}$ W, -VI-, Rutter 52 (MO); King Salmon, $58^{\circ} 41^{\prime}$ N, $156^{\circ} 39^{\prime} \mathrm{W}, 9-\mathrm{VII}-1952$, Schofield 2108 (WTU); Kodiak group, Raspberry island, Port Nita, $58^{\circ} 4^{\prime} \mathrm{N}, 153^{\circ} 10^{\prime} \mathrm{W}, 1-\mathrm{VII}-1945$, Eyerdam 3769 (MO); Kodiak Island, $57^{\circ} 42^{\prime} \mathrm{N}, 152^{\circ} 24^{\prime} \mathrm{W}, 20-\mathrm{VII}-1899$, Trelease \& Saunders 4370 (MO); Kodiak Island, Cheif Cove Meadow, S of Cove, $57^{\circ} 0^{\prime} \mathrm{N}, 154^{\circ} 0^{\prime} \mathrm{W}, 10-\mathrm{VIII}-1990$. Myers 67 (MO); Kodiak Island, Piller Mtn., above town of Kodiak, $57^{\circ} 47^{\prime}$ N, $152^{\circ} 26^{\prime}$ W, 20-VI-1992, Parker 3344 (MO); Kodiak Island, Valleys, Alitak, $56^{\circ} 53^{\prime} \mathrm{N}$, $154^{\circ} 7^{\prime} \mathrm{W}$, -VI-, Looff 127 (MO); Kuiu Isl., 56 $32^{\circ} \mathrm{N}$, $134^{\circ} 3^{\prime} \mathrm{W}, 3-\mathrm{VII}-1915$, Walker 769 (MO); Lake Iliamna region, $59^{\circ} 45^{\prime} \mathrm{N}, 154^{\circ} 54^{\prime} \mathrm{W}, 1902$, Gorman 64 (MO); Log Cabin, $63^{\circ} 1$ 'N, $143^{\circ} 20^{\prime} \mathrm{W}, 30-\mathrm{VII}-1907$, Cowles 971 (MO); Matanuska-Susitna Co., Curry, 62 $36^{\prime} \mathrm{N}$, $150^{\circ} 0^{\circ} \mathrm{W}, 9-\mathrm{VII}-1938$, Purer 7559 (MO); McKinley Na-

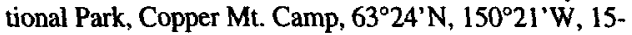
VII-1928, Mexia 2097 (MO); McKinley National Park, near Camp Eilson, mile 66, $62^{\circ} 29^{\prime} \mathrm{N}, 150^{\circ} 45^{\prime} \mathrm{W}, 11-$ VIII-1939, Nelson \& Nelson 4109 (MO); Middleton Is., $59^{\circ} 26^{\prime} \mathrm{N}, 146^{\circ} 19^{\prime} \mathrm{W}, 2$-VIII-1956, Thomas 6320 (WTU); mile 197 Richardson Highway, $63^{\circ} 10^{\prime} \mathrm{N}, 145^{\circ} 35^{\prime} \mathrm{W}, 3-$ VIII-1951, Webster 170 (DAO); near Palmer, 61 ${ }^{\circ} 35^{\prime} \mathrm{N}$, $149^{\circ} 6^{\prime} \mathrm{W}, 5$-VII-1943, York Pa 206 (MO); Palmer Creek, valley SE of Hope, Kenai Peninsula, $60^{\circ} 49^{\prime} \mathrm{N}$, 149 33'W, 26-VII-1951, Calder 6289 (DAO); Palmer Highway, $61^{\circ} 35^{\prime} \mathrm{N}, 149^{\circ} 6 \mathrm{~W}, 8$-VII-1946, Heller s.n. (WTU); Pribilof Is., St. Paul, $57^{\circ} 19^{\prime} \mathrm{N}, 170^{\circ} 2^{\prime} \mathrm{W}, 29$-VII1897, TKuneais s.n. (WTU); Prince of Wales-Outer Ketchikan Co., Fish Creek, beyond Hyder, 55 $55^{\prime} \mathrm{N}$, $130^{\circ} 1$ 'W, 29-VI-1924, Whited 1294 (MO); Valdez-Cordova Co., Evans Island, Port San Juan, $59^{\circ} 48^{\prime} \mathrm{N}$, 147 54 'W, 25-VII-1948, Eyerdam 7319 (MO); ValdezCordova Co., Harriman Fiord, $61^{\circ} 2^{\prime} \mathrm{N}, 148^{\circ} 19^{\prime} \mathrm{W}, 20-$ VIII-1935, Cooper 172 (F); Valdez-Cordova Co., Hinchinbrook, $60^{\circ} 14^{\prime} \mathrm{N}, 146^{\circ} 39^{\prime} \mathrm{W}, 30$-VI-1937, Eyerdam s.n. (MO); Valdez-Cordova Co., Virgin Bay, 60 $53^{\prime} \mathrm{N}$, 146 ${ }^{\circ}$ ' 'W, 25-VI-1899, Trelease \& Saunders 4367 (MO); Wrangell-Petersburg Co., Mt. Dewey, $56^{\circ} 28^{\prime} \mathrm{N}$, $132^{\circ} 23^{\prime}$ W, 2-VIII-1907, Cowles 1278 (MO). ALEUTIAN Is: Adak Island, hillside behind enlisted Navy personnel Barracks, 51 ${ }^{\circ} 52^{\prime}$ N, $176^{\circ} 39^{\prime}$ W, 7-VII-1974, Hein 106 (MO); Aleutians East Co., Nagai Island, Misty Harbor, $54^{\circ} 57^{\prime} \mathrm{N}, 160^{\circ} 9^{\prime} \mathrm{W}, 22-\mathrm{VI}-1893$, Townsend s.n. (F); Aleutians East Co., Shumagin Islands, Sand Point, $55^{\circ} 19^{\prime} \mathrm{N}, 160^{\circ} 31^{\prime} \mathrm{W}, 7-\mathrm{VII}-1899$, Trelease \& Saunders 4371 (MO); Attu Is., Sarana Bay, 52 $2^{\circ} 52^{\prime} \mathrm{N}, 173^{\circ} 17^{\prime} \mathrm{W}$, 22-VI-1945, Hardy 129 (WTU); Attu Island, Jarmin Pass, $52^{\circ} 56^{\prime} \mathrm{N}, 173^{\circ} 14^{\prime} \mathrm{W}$, 9-IX-1945, Soule 533 (MO); Chichagof Is., Khaz Penin., Point Slocum, 57 $27^{\circ} \mathrm{N}$, 135'54'W, 19-VI-1979, Muller 2914 (WTU); Cold Bay, $55^{\circ} 11^{\prime} \mathrm{N}, 162^{\circ} 34^{\prime} \mathrm{W}, 25$-VII-1952, Schofield 2383 (DAO); Lime Hills Quad, Cairn mt., $61^{\circ} 8^{\prime} \mathrm{N}, 155^{\circ} 16^{\prime} \mathrm{W}$, 28-VI-1999. Parker \& al. 8726 (MA); Naknek, 58 $43^{\prime}$ N,

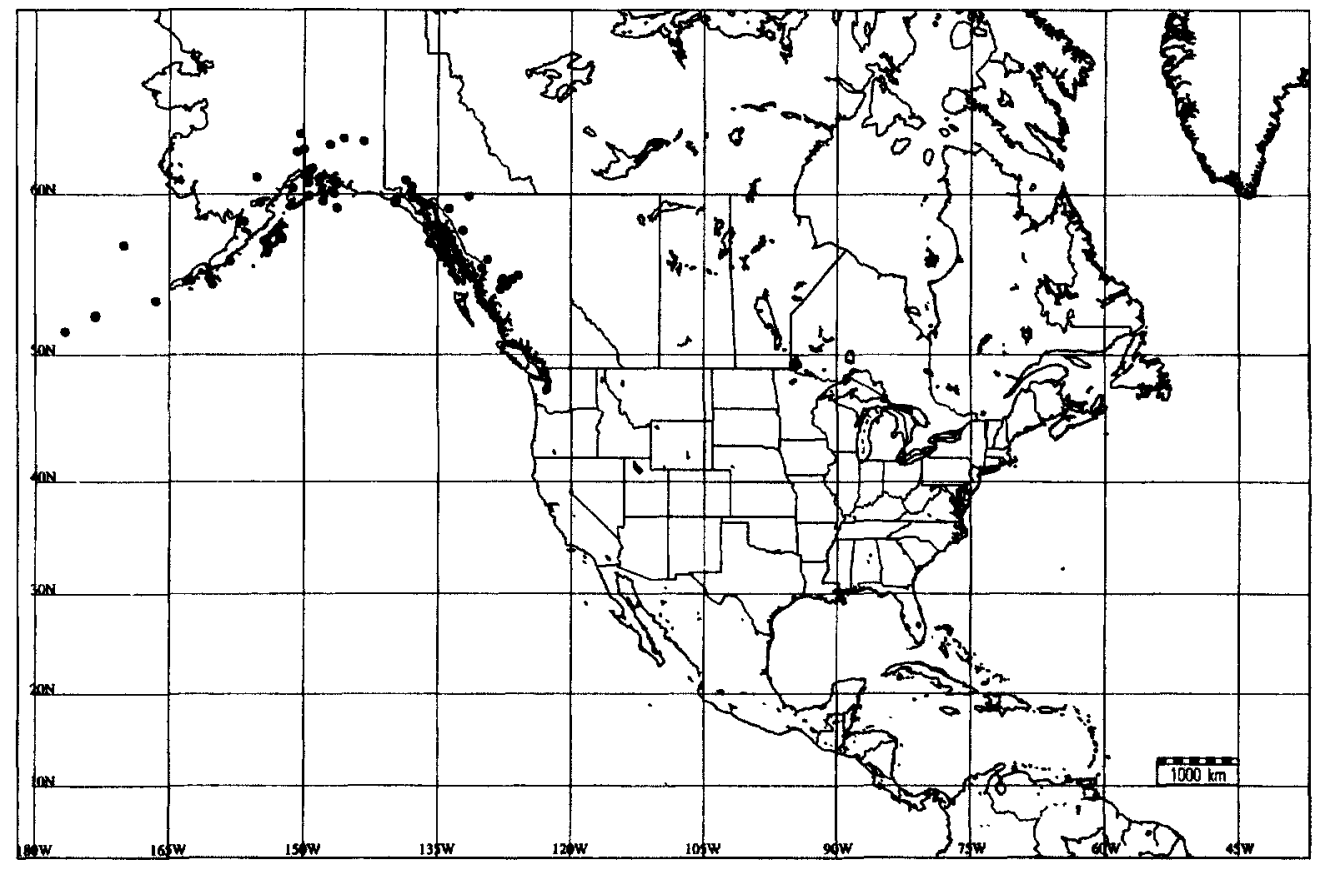

Fig. 3.-Distribution of Geranium erianthum in North America. 
$157^{\circ} 0^{\prime} \mathrm{W}, 3-\mathrm{VII}-1952$, Schofield 1938 (DAO); Unalaska, $53^{\circ} 52^{\prime} \mathrm{N}, 166^{\circ} 32^{\prime} \mathrm{W}, 25-\mathrm{VII}-1891$, Macoun 35 (MO).

2. Geranium maculatum L., Sp. Pl.: 681 (1753)

Ind. loc.: "Habitat in Carolina, Virginia, Sibiria"

Typus: USA. Georgia, Union Co., Cooper Creek, Utrech \& al. 82-030 (epitype, here designated, BM!; lectotype, here designated, Dillenius, Hort. Eltham. 1, tab. 132 fig. 159. 1732)

= G. maculatum var. albiflorum Raf., Med. Fl. 1:217 (1828), nom. nud.

G. maculatum f. albiflorum Raf. ex House in Bull. New York State Mus. Nat. Hist. 243-244: 48 (1923)

Ind. loc.: "Syracuse, Mrs M. O. Rust (State herbarium) and reported in 35th Rep't N. Y. State Museum 145. 1884"

Typus: USA. New York, Syracuse, Rust s.n. (no authentic material located)

$=$ G. maculatum var. diphyllum Raf., Med. Fl. 1: 217 (1828), nom. nud.

$=$ G. maculatum var. humile Raf., Med. Fl. 1: 217 (1828), nom. nud.

= G. maculatum var. macrophyllum Raf., Med. Fl. 1: 217 (1828), nom. nud.

= G. maculatum var. viride Raf., Med. Fl. 1: 217 (1828), nom. nud.

= G. maculatum var. album H.M. Myers in Amer. Naturalist 4: 438 (1870)

Ind. loc.: "Binghamton, N.Y."

Typus: no original material found

$=$ G. maculatum var. parviflora N. Coleman in Kent Sci. Inst. Misc. Publ. 2: 10 (1874) Ind. loc.: "Michigan"

Typus: USA. Michigan, $N$. Coleman s.n. [according to $\mathrm{C}$. Anderson, in litt., no material related to the protologue at $\mathrm{MICH}]$

$=$ G. maculatum var. plenum Lauman in L.H. Bailey, Cycl. Amer. Hort.: 640 (1900)

Ind. loc.: [not indicated]

Typus: no original material found

Herbs perennial, $18-70 \mathrm{~cm}$ tall. Rootstock 8$12 \mathrm{~mm}$ diam., horizontal, not tuberculate, not turnip-shaped. Stem erect, solitary, with patent or retrorse, eglandular hairs $0.3-0.6 \mathrm{~mm}$ long, and sometimes, patent, glandular hairs 1.5-1.7 $\mathrm{mm}$ long. Basal leaves in a persistent rosette; lamina 5-11.5 $\times 7-14.5 \mathrm{~cm}$, polygonal in outline, cordate, palmatifid (divided for $0.74-0.83$ of its length), pilose, with appressed, eglandular hairs; segments 5 , rhombic, $5-12 \mathrm{~mm}$ at the base, (8-)10-20-lobed in distal half (ratio mainsinus length of the middle segment / middlesegment length $=0.1-0.21$ ); cauline leaves opposite; petioles to $33 \mathrm{~cm}$ long, with patent to retrorse, eglandular hairs $0.5-0.6 \mathrm{~mm}$ long, and sometimes patent, glandular hairs $1.1-1.5 \mathrm{~mm}$ long; stipules 8-13 $\times 1-2 \mathrm{~mm}$, linear-lanceolate, with glandular and eglandular hairs on abaxial surface and on the margin, glabrous adaxially. Inflorescence dichasial cyme; cymules 2-flowered, solitary or in aggregates at the top of each branch; peduncles (0-)2-9 cm long, with patent to retrorse, eglandular hairs $0.2-0.9 \mathrm{~mm}$ long, rarely with glandular hairs ca. $1.5 \mathrm{~mm}$ long; bracteoles 4-5 $\times 1 \mathrm{~mm}$, linear-lanceolate, with eglandular and glandular hairs on abaxial surface and on the margin, glabrous adaxially; pedicels $1-3.1 \mathrm{~cm}$ long, with patent to retrorse, eglandular hairs 0.2-0.9 mm long; pedicel and peduncle together often overtopping the subtending leaf. Sepals 6-9 × 3-4 mm (ratio pedicel length / sepal length $=1.4-4.6$ ), smooth, not accrescent, 3-5-nerved, with mucro $1.5-3 \mathrm{~mm}$ long, with scarious margins $0.1-0.2 \mathrm{~mm}$ wide, with antrorse, appressed eglandular hairs 0.2$0.6 \mathrm{~mm}$ long and patent, glandular hairs 0.9-1.2 $\mathrm{mm}$ long on the abaxial side, glabrous adaxially. Petals $12-16 \times 8.5-12 \mathrm{~mm}$ (ratio petal length / petal width $=1.3-1.4$ ), erect-patent, entire, without claw, glabrous on both sides, ciliate on the basal margin, purplish, sometimes white. Stamens 10 , both whorls bearing anthers; filaments 8-9 mm long, not exserted, lanceolate, pilose on the abaxial side, ciliate on the proximal half, with hairs $0.6 \mathrm{~mm}$ long; anthers $2 \times 1$ $\mathrm{mm}$. Nectaries with a tuft of hairs at the top, dorsally glabrous. Gynoecium 11-12 mm long. Fruit 27-33 mm long, erect when immature; mericarps 3.5-4 $\times 2-2.5 \mathrm{~mm}$, without a strand of fibres, smooth, without longitudinal rib, without basal beak, with a basal callus, with \pm patent, eglandular hairs $0.2-0.6 \mathrm{~mm}$ long, and glandular 1.1-1.7 mm long, brownish; rostrum $17-31 \mathrm{~mm}$ long, with a narrowed apex 5-7 mm long, with erect-patent, eglandular hairs ca. 0.1 
mm long; stigmatic remains 2-2.5 mm long (ratio fruit length / stigmatic remains length $=13-$ 16), with 5 glabrous lobes. Seeds $2.5 \times 1.8 \mathrm{~mm}$, reticulate, brownish; hilum $1 / 4$ as long as the perimeter. Cotyledons entire. $n=26$.

Flowering March-July (exceptionally September). Woods, sometimes meadows or roadsides; 0-950 m. Eastern Canada, and North Central, Northeastern and Southeastern U.S.A. Fig. 6.

Illustrations. Fig. 1c, 1e, 4, 5b. SweET (1826-28, tab. 9); YEO (1992a: 122 fig. 9.58), Cavanilles (1787, tab. 86 fig. 2).

Geranium maculatum is readily distinguished from all other species treated here by the combination of the following three characters: long petals glabrous on the adaxial surface, pedicels only with eglandular hairs, and nectaries with a tuft of hairs at the top. This species shows long glandular hairs ending in a usually reddish narrow cell. These hairs are always present on sepals and mericarp apex, usually on stipules and bracteoles, and exceptionally on petioles and peduncles.

Geranium maculatum is similar to $G$. erianthum, $G$. sylvaticum, and $G$. oreganum by virtue of its glabrous petals on the adaxial surface, but differs most obviously from G. erianthum and $G$. sylvaticum by its nectaries with a tuft of hairs at the top, and from $G$. oreganum by its eglandular pedicels.

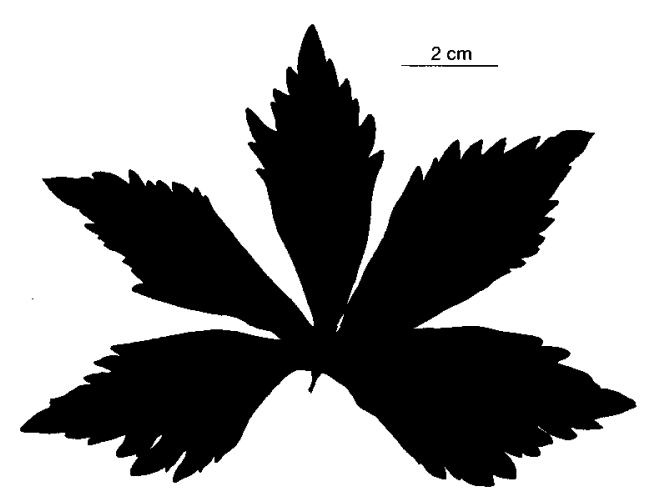

Fig. 4.-Silhouette of lamina of Geranium maculatum [Wharton $3700(\mathrm{MO})]$.
According to JoNES \& JONES (1943: 13) $G$. maculatum is the most common perennial species of eastern North America. It seems to be geographically isolated from $G$. oreganum, and $G$. erianthum, both growing in western North America.

The specimens supporting the record of $G$. maculatum from South Dakota (BRUGGEN 1985: 301) have not been studied.

\section{Representative specimens examined}

CANADA. ONTARIO: at road cut-off of Hwy 401 with Salmon River, $44^{\circ} 12^{\prime} \mathrm{N}, 77^{\circ} 13^{\prime} \mathrm{W}, 30-\mathrm{V}-1967$, Baum 67 05-30-0 (DAO); Bathurst Ravine, between Spadina and Bathurst, Toronto, $43^{\circ} 42^{\prime} \mathrm{N}, 79^{\circ} 25^{\prime} \mathrm{W}, 3-\mathrm{VI}-1956$, Fleischmann 201 (DAO); Bothwell, 42 $38^{\circ} \mathrm{N}, 81^{\circ} 52^{\prime} \mathrm{W}, 27-\mathrm{V}-$ 1933, Stirrett s.n. (DAO); Brant Co., 3 mi SE of Glen Morris, $43^{\circ} 16^{\prime} \mathrm{N}, 80^{\circ} 2 \mathrm{I}^{\prime} \mathrm{W}, 27-\mathrm{V}-1950$, Soper \& Shields 4434 (MO); Cartwright Point, $44^{\circ} 14^{\prime} \mathrm{N}, 76^{\circ} 27^{\prime} \mathrm{W},-1886$, Fowler s.n. (DAO); Essex Co., LaSalle, $42^{\circ} 13^{\prime} \mathrm{N}, 8^{\circ} 3^{\prime} \mathrm{W}, 1-\mathrm{V}$ 1955, Rogers 10061 (DAO); Grand Bend Pinery, on highway $21,43^{\circ} 19^{\prime} \mathrm{N}, 81^{\circ} 45^{\prime} \mathrm{W}, 28-\mathrm{V}-1956$, Fleischmann 33 (DAO); Grenville Co., Prescott, $44^{\circ} 43^{\prime} \mathrm{N}, 75^{\circ} 31^{\prime} \mathrm{W}, 28-$ VIII-1960, Dore 18134 (DAO); Haldiman Co., Dunnville, $42^{\circ} 54^{\prime} \mathrm{N}, 79^{\circ} 36^{\prime} \mathrm{W}, 18-\mathrm{V}-1951$, Miller 57 (DAO); Halton Co., Bronte Creek, $3 \mathrm{mi}$ W of Palermo, $43^{\circ} 25^{\prime} \mathrm{N}, 79^{\circ} 46^{\prime} \mathrm{W}$, 17-V-1974, Catling \& al. 3924 (DAO); Kilworth, 42 $58^{\circ}$ N, 812' W, 21-V-1934, Wood s.n. (DAO); Leeds Co., $10 \mathrm{mi}$ SW Brockville, $44^{\circ} 35^{\prime} \mathrm{N}, 75^{\circ} 41^{\prime} \mathrm{W}, 7-\mathrm{VI}-1955$, Dore \& Gillette 15798 (DAO); Lincoln Co., De Cew Falls, 43ํ'N, $79^{\circ} 16^{\prime} \mathrm{W}, 9-\mathrm{V}-1952$, Miller 42 (DAO); Middlesex Co., Komoka, 42 $57^{\circ}$ N, $81^{\circ} 26^{\prime} \mathrm{W}, 22-\mathrm{V}-1960$, Minshall 4386 (DAO); Norfolk Co., Port Dover, $42^{\circ} 47^{\prime} \mathrm{N}, 80^{\circ} 12^{\prime} \mathrm{W}, 21$ XII-1949, Welch 3 (DAO); Northumberland Co., Hamilton, $44^{\circ} 5^{\prime} \mathrm{N}, 78^{\circ} 19^{\prime} \mathrm{W}, 29-\mathrm{V}-1975$, Riley \& Hoy 3924 (DAO); Perth Co., Stratford, $43^{\circ} 22^{\prime} \mathrm{N}, 80^{\circ} 57^{\prime} \mathrm{W}, 26$-VI1911, Beamer s.n. (DAO); Presquile, S of Brighton,

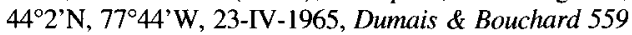
(DAO); Prince Eduard Co., Glenora, $44^{\circ} 2^{\prime} \mathrm{N}, 77^{\circ} 3^{\prime} \mathrm{W}, 21-$ VIII-1957, Dore 16922 (DAO); Queenston, 4310'N, 79³'W, 22-V-1952, Miller 154 (DAO); Scugog, Pine Point Woods, $44^{\circ} 8^{\prime} \mathrm{N}, 78^{\circ} 55^{\prime} \mathrm{W}, 17-\mathrm{VI}-1961$, Tozer 9 (DAO); Simcoe Co., Bradford, $44^{\circ} 7^{\prime} \mathrm{N}, 79^{\circ} 34^{\prime} \mathrm{W}, 2$-VI1974, Bobbette 4132 (DAO); Welland Co., Pelham Twp, $43^{\circ} 3^{\prime} \mathrm{N}, 7^{\circ} 21^{\prime} \mathrm{W}, 28-\mathrm{V}-1948$, Soper \& Dale 3759 (MO). QuÉBEC: Vaudreuil Co., Ile Perrot, $45^{\circ} 22^{\prime} \mathrm{N}, 7^{\circ} 57^{\prime} \mathrm{W}, 13-$ VI-1924, Loius-Marie s.n. (DAO).

USA. AlaBAma: Dekalb Co., N of Fort Payne, $36^{\circ} 26^{\prime} \mathrm{N}$, 85'43'W, 27-IV-1936, Svenson 7702 (MO); Franklin Co., Russellville, $34^{\circ} 30^{\prime} \mathrm{N}, 87^{\circ} 43^{\prime} \mathrm{W}$, -IV-, James 12 (MO); Jackson Co., by Ala 65, 5.1-7.1 mi S Huntland, 34'52'N, 86¹1'W, 18-IV-1971, Kral 42099(MO); Marengo Co., by US 80, $1.2 \mathrm{mi}$ E Demopolis, $32^{\circ} 31^{\prime} \mathrm{N}, 87^{\circ} 50^{\prime} \mathrm{W}, 31-$ III1972, Kral 45407 (MO); Morgan Co., Cave Springs, E of Dekatur off River Road, 34 32'N, 86 $51^{\circ}$ 'W, 28-III-1973, Kral 49423 (MO); Perry Co., by Ala. 219, 14.5 mi S Bibb Co. line, $32^{\circ} 45^{\prime}$ N, $87^{\circ} 17^{\prime} \mathrm{W}$, 1-IV-1972, Kral 45458 (MO); Talladega Co., by US $280,1 \mathrm{mi}$ SE Sylacauga, $33^{\circ} 10^{\prime} \mathrm{N}$, 86 $15^{\circ}$ 'W, 30-IV-1972, Kral 46044 (MO). ARKANSAS: Gar- 
land Co., Crystal Springs, 34³1'N, 93'20'W, 26-IV-1941, Demaree 21977 (MO); Greene Co., Crowleys Ridge, $36^{\circ} 2^{\prime} \mathrm{N}, 90^{\circ} 39^{\prime} \mathrm{W}, 23-\mathrm{IV}-1950$, Demaree 28770 (OKL); Hot Springs Co., Magnet Cove, $34^{\circ} 27^{\prime} \mathrm{N}, 92^{\circ} 50^{\prime} \mathrm{W}, 4-\mathrm{IX}-$ 1938, Demaree 16940 (MO); Logan Co., Magazine mountain, Ozark National Forest, 3548’N, 9333' W, 5-IX-1942, Demaree 22837 (MO); Marion Co., near Buffalo River, 16 mi SSE of Yellville, $35^{\circ} 49^{\prime} \mathrm{N}, 93^{\circ} 27^{\prime} \mathrm{W}, 2-\mathrm{V}-1940$, Hubricht B1759 (MO); Pope Co., Nogo, 35³8'N, 92 52 'W, 5-IV-1933, Merrill 129 (MO). ConNecticut:

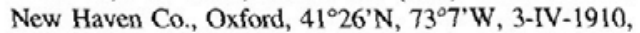
Overholts s.n. (MO). Delaware: New Castle Co., Greenbank, 394 'N, 75'38'W, 22-V-1884, Commons s.n. (MO). DisTRICT OF COLUMBIA: Washington, $38^{\circ} 53^{\prime} \mathrm{N}, 77^{\circ} 2^{\prime} \mathrm{W}, 20$ V-1878, Chickering s.n. (MO). GEORGIA: Bartow Co., small stream E of Allatoona Creek, W of Acworth, 33 ${ }^{\circ} 57^{\prime} \mathrm{N}$, 84'43'W, 17-IV-1948, Duncan 7987 (MO); Bibb Co., 7 mi
SE of Macon, $32^{\circ} 49^{\prime} \mathrm{N}, 83^{\circ} 37^{\prime} \mathrm{W}, 12-\mathrm{IV}-1940$, Duncan 1912 (MO); Clarke Co., Athens, $33^{\circ} 57^{\prime}$ N, $83^{\circ} 22^{\prime} \mathrm{W}, 12$-IV1917, Barfield 8 (OKL); Floyd Co., Rome, $34^{\circ} 15^{\prime} \mathrm{N}$, $85^{\circ} 9^{\prime} \mathrm{W}$ (MO); Monroe Co., High Falls State Park, $33^{\circ} 10^{\prime} \mathrm{N}$, 84 ${ }^{\circ} 1$ 'W , 8-IV-1988, Howel 1159 (MO); Walker Co., near Maddox Gap, between Villanow and La Fayette, $34^{\circ} 40^{\prime} \mathrm{N}$, 856'W, 8-IV-1988, Cronquist 1159 (MO); Washington Co., NW of Tennille, $32^{\circ} 55^{\prime} \mathrm{N}, 83^{\circ} 50$ 'W, 11-IV-1904, Harper 2112 (MO). ILLINOIS: Calhoun Co., Batchtown water tower, $39^{\circ} 1^{\prime} \mathrm{N}, 90^{\circ} 39^{\prime} \mathrm{W}, 17-\mathrm{IV}-1981$, Ugent \& al. 81-12 (MO); Champaign Co., Mahomet, $40^{\circ} 11^{\prime} \mathrm{N}, 88^{\circ} 24^{\prime} \mathrm{W}, 28$ IV-1946, Rapp s.n. (MO); Cook Co., Maywood, 41 52 'N, 8750'W, 26-V-1897, Chase s.n. (MO); Henderson Co., 2 mi E of Oquaka, Jinks Hollow, 40 56 ' N, 90 56 'W, 16-V1971, Solomon 39 (MO); Jackson Co., Natural Bridge, $37^{\circ} 38^{\prime} \mathrm{N}, 89^{\circ} 20^{\prime} \mathrm{W}, 26-\mathrm{V}-1923$, McFarland 71 (MO); Knox Co., $5.5 \mathrm{mi} \mathrm{S}$ and $2 \mathrm{mi}$ E of Victoria, $41^{\circ} 2^{\prime} \mathrm{N}, 90^{\circ} 5^{\prime} \mathrm{W}$,
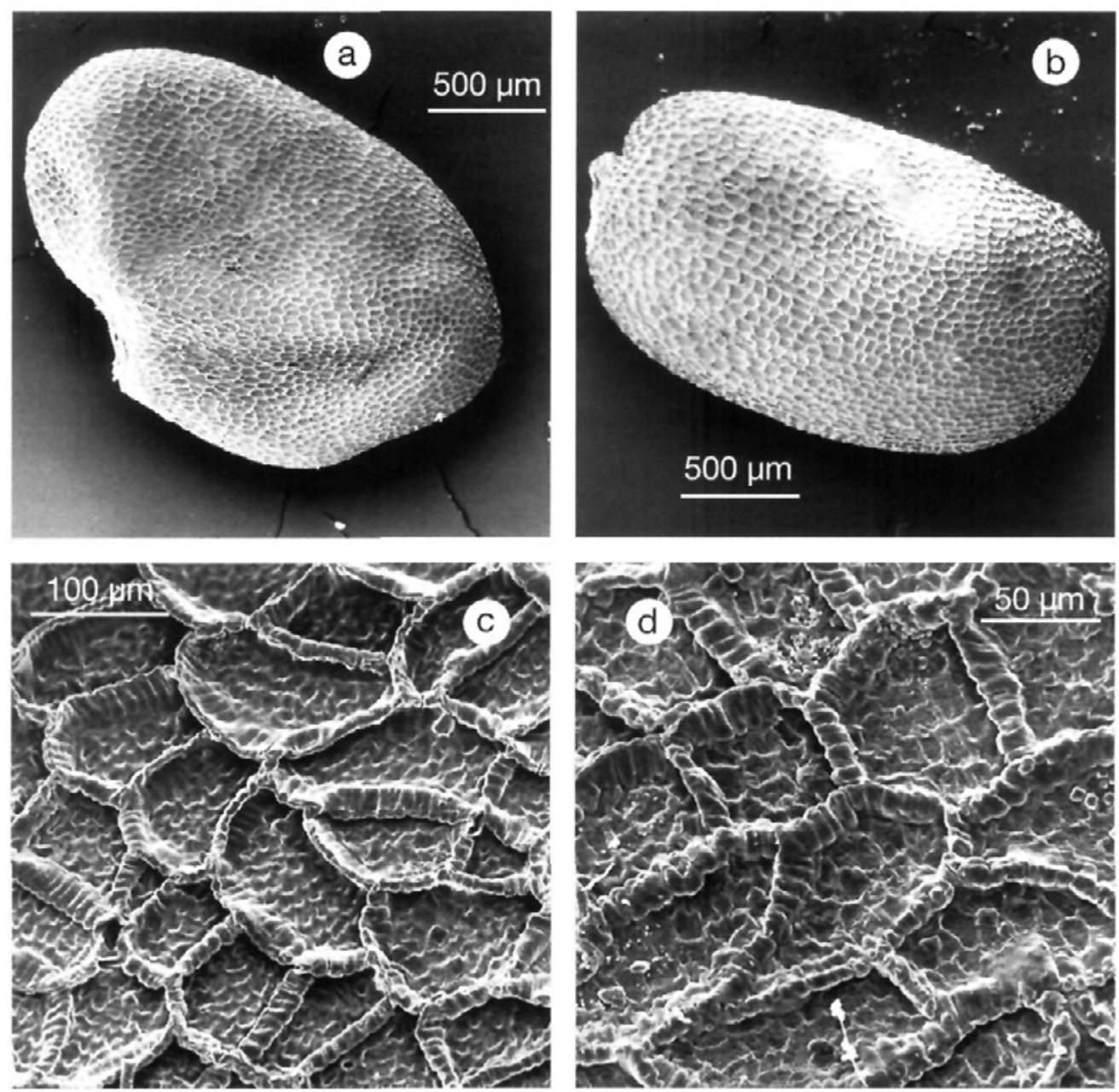

Fig. 5.-Seeds SEM photomicrographs of: a, Geranium erianthum [Calder 14736 (DAO)]; b, G. maculatum [Fleischmann 88 (DAO)]; c, G. caespitosum [Brunt I6 (NMC)]; d, G. wislizenii [Shreve 6324 (NMC)]. 


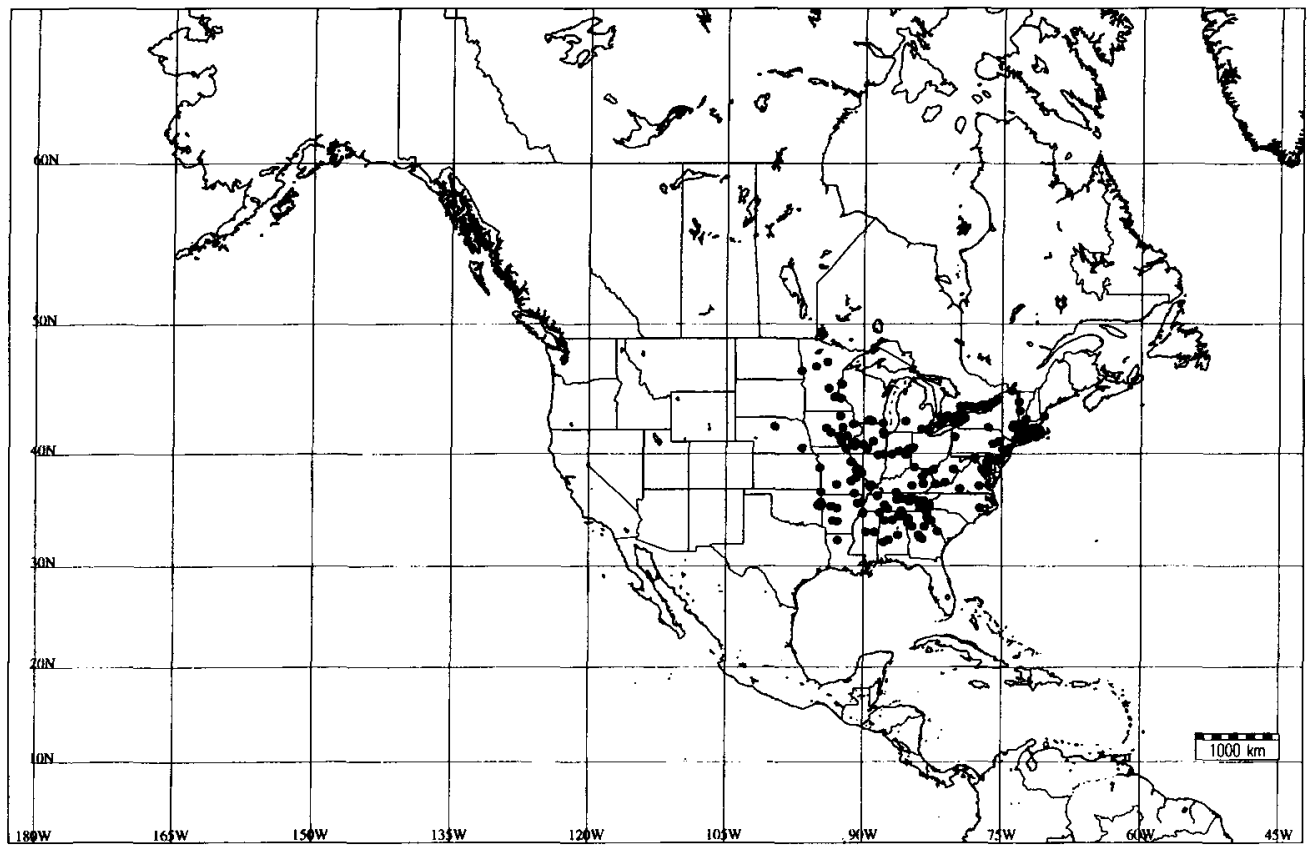

Fig. 6.-Distribution of Geranium maculatum.

5-V-1971, Solomon 26 (MO); La Salle Co., Starved Rock, $41^{\circ} 19^{\prime} \mathrm{N}, 88^{\circ} 59^{\prime} \mathrm{W}, 1-\mathrm{VI}-1909$, Greenman \& al. 30 (MO); Mercer Co., along Edwards River, $\mathrm{N}$ of Joy, $41^{\circ} 11^{\prime} \mathrm{N}$, 90 $52^{\prime}$ W, 9-V-1968, Evers 94363 (MO); Peoria Co., Peoria Heights, $40^{\circ} 41^{\prime} \mathrm{N}, 89^{\circ} 35^{\prime} \mathrm{W}, 25-\mathrm{V}-1919$, Chase 3087 (MO); Union Co., Anna, 37'29'N, 89 $16^{\circ} \mathrm{W}, 18$-IV-1919, Palmer 14860 (MO); Vermilion Co., along Vermilion River between Oakwood and Collison, $40^{\circ} 13^{\prime} \mathrm{N}, 87^{\circ} 48^{\prime} \mathrm{W}$, 18 $\mathrm{V}$-1940, Jones 11272 (MO); Willianson Co., near New Dam Site on Grass Creek at Devils Kitchen, $37^{\circ} 38^{\prime} \mathrm{N}, 89^{\circ} 6^{\prime} \mathrm{W}$, 21-IV-1941, McCree 692 (MO). Indiana: Delaware Co., $40^{\circ} 11^{\prime} \mathrm{N}, 85^{\circ} 23^{\prime} \mathrm{W}, 26-\mathrm{IV}-1954$, Humbles 65 (MO); Howard Co., 4 mi NW of Kokomo, $40^{\circ} 29^{\prime} \mathrm{N}, 86^{\circ} 8^{\prime} \mathrm{W}, 5-\mathrm{V}-$ 1942, Ek s.n. (MO); Tippecanoe Co., 5 mi E of Romney, $40^{\circ} 14^{\prime} \mathrm{N}, 86^{\circ} 54^{\prime} \mathrm{W}, 22-\mathrm{V}-1960$, Venrick 124 (MO); Wells Co., Harrison, $40^{\circ} 41^{\prime} \mathrm{N}, 85^{\circ} 8^{\prime} \mathrm{W}, 14-\mathrm{V}-1905$, Deam s.n. (MO). Iowa: Black Hawk Co., 42 $28^{\prime} \mathrm{N}, 92^{\circ} 19^{\prime} \mathrm{W}, 9-\mathrm{V}-$ 1929, Burk 242 (MO); Clayton Co., Osterdock, 42 $44^{\prime} \mathrm{N}$, 91 ${ }^{\circ}$ 'W, 15-V-1920, Shimek s.n. (MO); Iowa Co., N of Homestead, $41^{\circ} 45^{\prime}$ N, $91^{\circ} 52^{\prime} \mathrm{W}, 24-\mathrm{V}-1913$, Shimek s.n. (MO); Louisa Co., along highway embankment, 41 $14^{\prime} \mathrm{N}$, 91 ${ }^{\circ} 16^{\prime} \mathrm{W}, 22-\mathrm{V}-1937$, Goodman 278I (MO); Mahaska Co., Moorhead, $41^{\circ} 19^{\prime} \mathrm{N}, 92^{\circ} 27^{\prime} \mathrm{W}, 7-\mathrm{V}-1938$, Augustine $57 \mathrm{a}$

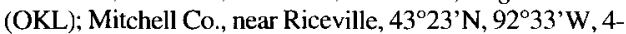
VII-1947, Johstead 9870 (MO); Poweshiek Co., Grinnell, $41^{\circ} 44^{\prime} \mathrm{N}, 92^{\circ} 43^{\prime} \mathrm{W}, 10-\mathrm{V}-1906$, W.E.J. s.n. (OKL); Story Co., Ames, 42 $2^{\prime} \mathrm{N}, 93^{\circ} 37^{\prime} \mathrm{W}, 29-\mathrm{V}-1908$, Jeffs s.n. (OKL); Van Buren Co., Bentonsport, $40^{\circ} 43^{\prime} \mathrm{N}, 91^{\circ} 51^{\prime} \mathrm{W},-\mathrm{V}-$, Graves 1780 (MO); Webster Co., Woodmans Hollow, $42^{\circ} 25^{\prime} \mathrm{N}, 94^{\circ} 6^{\prime} \mathrm{W}, 30-\mathrm{V}-1904$, Churchill 2239 (NEB).

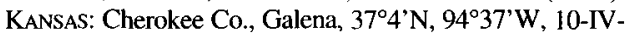

1938, Horr \& McGregor E187 (OKL); Wyandotte Co., 39 $7^{\circ}$ N, 94 ${ }^{\circ} 43^{\prime} \mathrm{W}, 1897$, Hitchcock 1138 (MO). KENTUCKY: Bowling Green, $36^{\circ} 59^{\prime} \mathrm{N}, 86^{\circ} 26^{\prime} \mathrm{W},-\mathrm{V}-$, Price s.n. (MO); Calloway Co., Backusburg, $36^{\circ} 42^{\prime} \mathrm{N}, 88^{\circ} 28^{\prime} \mathrm{W}, 27-\mathrm{IV}$ 1973, Athey 2242 (MO); Casey Co., near Willow Grove, $37^{\circ} 35^{\prime} \mathrm{N}, 84^{\circ} 43^{\prime} \mathrm{W}, 1-\mathrm{V}-1939$, Wharton 3700 (MO); Fleming Co., $3.5 \mathrm{mi}$ SE of Goddard, $38^{\circ} 21^{\prime} \mathrm{N}, 83^{\circ} 36^{\prime} \mathrm{W}, 12-\mathrm{V}$ 1939, Wharton 3833 (MO); Wolfe Co., between markers 54 and 55 on Mountain Parkway on S side of road, $37^{\circ} 44$ 'N, 832' W, 26-IV-1964, Bevins 77 (MO). LOUISLANA: Lincoln Co., E of Sugar Creek, $32^{\circ} 42^{\prime} \mathrm{N}, 92^{\circ} 48^{\prime} \mathrm{W}, 7-\mathrm{V}-1970$, Thomas 18428 (MO). MaINE: York Co., Kennebunkport, $43^{\circ} 21^{\prime}$ N, $70^{\circ} 28^{\prime}$ W. 7-VIl-1915, Pier 86 (MO). MARYLAND: Baltimore Co., at tributary of Big Gunpowder River, $\mathrm{W}$ of Big Falls Road near Monkton, 39 34'N, 76 $36^{\prime} \mathrm{W}, 11-\mathrm{V}$ 1981, Hill 10005 (MO); Prince Georges Co., College Park, $38^{\circ} 58^{\prime} \mathrm{N}, 76^{\circ} 56^{\prime} \mathrm{W}, 8-\mathrm{V}-1900$, Steward s.n. (MO). MASSA-

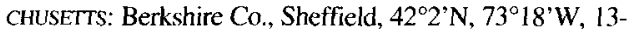
VI-1919, Churchill s.n. (MO). MiCHIGAN: Ingham Co., Alaiedon Twp, Wt side of Phillips Road, $0.5 \mathrm{mi} \mathrm{N}$ of Willoughby Road, 16-V-1953, Gilly \& al. 7428 (MO); Kent Co., between the Thornapple River and 100th Street, $42^{\circ} 57^{\prime} \mathrm{N}, 85^{\circ} 28^{\prime} \mathrm{W}, 28-\mathrm{V}-1973$, Davidse 3310 (MO); Washtenaw Co., Ann Arbor, $42^{\circ} 17^{\prime} \mathrm{N}, 83^{\circ} 44^{\prime} \mathrm{W}, 24-\mathrm{V}-$ 1937, Hermann 8589 (MO). Minnesota: Clay Co., Moorhead, $46^{\circ} 52^{\prime} \mathrm{N}, 96^{\circ} 46^{\prime} \mathrm{W}, 12-\mathrm{VII}-1912$, Stevens s.n. (OKL); Clearwater Co., Itasca Park, $47^{\circ} 11^{\prime} \mathrm{N}, 95^{\circ} 12^{\prime} \mathrm{W}, 14-\mathrm{VII}-$ 1933, Moyle 804 (MO); Hennepin Co., Fort Snelling,

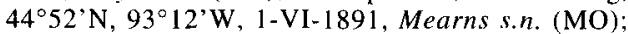
Koochiching Co., Wildwood, White Bass Lake, $47^{\circ} 32^{\prime} \mathrm{N}$, 93⒌'W, 21-VI-1905, Cleburne s.n. (NEB); Ramsey Co., 
Saint Paul, $44^{\circ} 56^{\prime} \mathrm{N}, 93^{\circ} 5^{\prime} \mathrm{W}$, Dewart 88 (NEB); Sherburne Co., Santiago, $45^{\circ} 32^{\prime} \mathrm{N}, 93^{\circ} 49^{\prime} \mathrm{W}, 12-\mathrm{VI}-1971$, McCollum $l$ (NEB). MississIPPI: Montgomery Co., Winona, $33^{\circ} 28^{\prime} \mathrm{N}$, 89.43'W, -VI-, Freiberg s.n. (MO); Oktibbeha Co., Starkville, $33^{\circ} 27^{\prime} \mathrm{N}, 88^{\circ} 49^{\prime} \mathrm{W}, 5$-IV-1897, Tracy s.n. (MO). MissourI: Carter Co., Big Spring, 36 $57^{\circ} \mathrm{N}, 9^{\circ} 59^{\prime} \mathrm{W}, 19-$ IV-1970, Sutter 55 (MO); Crawford Co., along state route 8, $14 \mathrm{mi}$ E of Steelville at Cortois Creek, $37^{\circ} 58^{\prime} \mathrm{N}, 91^{\circ} 21^{\prime} \mathrm{W}, 1$ V-1990, Miller \& al. 4919 (MO); Dallas Co., Windyville, $37^{\circ} 42^{\prime} \mathrm{N}, 92^{\circ} 55^{\circ} \mathrm{W}, 5-\mathrm{V}-1967$, Henderson $67-375$ (VT); Franklin Co., Meramec, $38^{\circ} 15^{\prime} \mathrm{N}, 9^{\circ} 48^{\prime} \mathrm{W}, 11-\mathrm{IV}-1973$, Sharp 136 (MO); Ralls Co., pr. Lick Creek, 39 $37^{\prime}$ N, 91'19'W, 3-VI-1974, Hudson 180 (MO); St. Charles Co., near Old Monroe, $38^{\circ} 55^{\prime} \mathrm{N}, 90^{\circ} 44^{\prime} \mathrm{W}, 12-\mathrm{V}-1917$, Davis 7239 (MO). NeBrasKa: Brown Co., Long Pine, $42^{\circ} 32^{\prime} \mathrm{N}$, 99.42'W, 23-V-1900, Bates s.n. (NEB); Lancaster Co., $40^{\circ} 47^{\prime} \mathrm{N}, 96^{\circ} 4 \mathrm{I}^{\prime} \mathrm{W}, 18-\mathrm{VI}-1909$, Martin s.n. (NEB). NEW HAMPSHIRE: Cheshire Co., Walpole, $43^{\circ} 8^{\prime} \mathrm{N}, 72^{\circ} 26^{\prime} \mathrm{W}, 23-$ V-1977, Boufford 5496 (MO). NEw JERSEY: Hunterdon Co., near High Bridge, $40^{\circ} 40^{\prime}$ N. $74^{\circ} 53^{\prime}$ W, 13-VI-1914, Fisher s.n. (MO). New York: Albany Co., Loudonville, $42^{\circ} 42^{\prime} \mathrm{N}$, 734ㄴ, W, 5-VI-1939. House s.m. (MO); Albany Co., S side of Hannacrois Creek, $42^{\circ} 28^{\circ} \mathrm{N}, 73^{\circ} 54^{\prime}$ 'W, 28-V-1990, Crins 8061 (MO); Tompkins Co., Ithaca, $42^{\circ} 26^{\prime} \mathrm{N}, 76^{\circ} 29^{\prime} \mathrm{W}, 4-$ VI-1891, Schrenk s.n. (MO). NORTH CAROLINA: Jackson Co., SE of hydroelectric plant. S of Tuckasegee, $35^{\circ} 16^{\prime} \mathrm{N}$, $83^{\circ} 7^{\prime} \mathrm{W}, 2$-V-1991, Hill 22202 (MO); Madison Co.. $3 \mathrm{mi}$ S of juntion of NC $209+\mathrm{NC} 63$ on NC $209,35^{\circ} 50^{\prime} \mathrm{N}$, 82 $2^{\circ} 49^{\prime}$ W, 9-V-1976, Solomon 5452 (MO); Pitt Co., Biltmore, $35^{\circ} 36^{\prime} \mathrm{N}, 77^{\circ} 23^{\prime} \mathrm{W}, 11$-VI-1897 (MO). NORTH Dakota: Cass Co., $1.5 \mathrm{mi} \mathrm{N}$ of Fargo, 46 ${ }^{\circ} 52^{\prime} \mathrm{N}, 96^{\circ} 47^{\prime} \mathrm{W}$, 12-VI-1970, Kukla 17 (MO). OHo: Hamilton Co., Cincin-

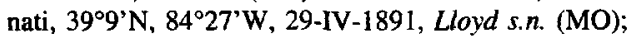
Scioto Co.. Shawnee State Forest, Camp Gordon, $38^{\circ} 43^{\prime} \mathrm{N}$, $83^{\circ} 12^{\prime} \mathrm{W}, 15-\mathrm{V}-1934$, Demaree 10618 (MO); Van Wert Co., Ohio, $40^{\circ} 46^{\prime} \mathrm{N}, 84^{\circ} 36^{\prime} \mathrm{W}, 1837$, Frank s.n. (MO); Vinton Co., Clinton Twp., lake Alma State Park, W of Little Raccoon Creek, 38 $57^{\circ} \mathrm{N}, 8^{\circ} 21^{\prime} \mathrm{W}, 18-\mathrm{IV}-1965, O^{\prime}$ Dell 113 (MO). OKLahoma: Adair Co., Stilwell, $35^{\circ} 48^{\prime} \mathrm{N}$, 94³7'W, 27-IV-1968, Perino \& Pierson 184 (OKL); Cherokee Co., Tahlequah, 35⒌' N, 94 $58^{\prime}$ 'W, 30-IV-1928, Little 29 (OKL); Delaware Co.. Dripping ' Springs, $36^{\circ} 10^{\prime} \mathrm{N}, 94^{\circ} 40^{\prime} \mathrm{W}, 6-\mathrm{V}-1958$, Wallis 6569 (OKL). PENNSYlvania: Crawford Co., Meadville Bousson Forest, $41^{\circ} 38^{\prime} \mathrm{N}, 80^{\circ} 9^{\prime} \mathrm{W}, 26-\mathrm{V}-1989$, Mellon 27 (MO): Delaware Co., along Crum Creek, $1 \mathrm{mi}$ N of Swarthmore. $39^{\circ} 54^{\prime} \mathrm{N}$, 75'21'W, 11-V-1933, Hymes s.n. (MO); Franklin Co., Fort Loudon, 39 $54^{\prime}$ N, $77^{\circ} 54^{\prime}$ W, 20-VI-1924, Pennell 12179 (MO); Lancaster, $40^{\circ} 2^{\prime} \mathrm{N}, 76^{\circ} 18^{\prime} \mathrm{W}, 5-\mathrm{VI}-1874$, Stevens s.n. (MO); Luzerne Co., Slocum, $41^{\circ} 7^{\prime} \mathrm{N}, 76^{\circ} 2^{\prime} \mathrm{W}, 13-\mathrm{VI}-$ 1935, Hughes 6620 (MO); near Philadelphia, 3957'N, $75^{\circ} 9^{\prime}$ W, - 1889, Greenman 858 (MO); Pike Co., Milford, $41^{\circ} 19^{\prime} \mathrm{N}, 74^{\circ} 84^{\prime} \mathrm{W}, 1$-VI-1908, [illegible] (MO). RHODE IsLAND: Greenwich, $41^{\circ} 40^{\prime} \mathrm{N}, 71^{\circ} 24^{\prime} \mathrm{W}, 27-\mathrm{V}-1877$, Congdon s.n. (P). SOUTH CAROLINA: Aiken Co., Hammond Hills,

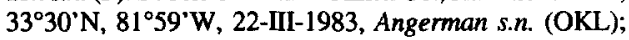
Anderson Co, Anderson, $34^{\circ} 30^{\prime} \mathrm{N}, 82^{\circ} 39^{\prime} \mathrm{W}, 19-\mathrm{IV}^{\prime}-1920$, Davis s.n. (MO); Oconee Co., Cleman College, $34^{\circ} 46^{\prime} \mathrm{N}$, 835' 'W, 5-IV-1906, House 1772 (MO). TENNESSEE: Blount Co., Kinzel Spring, 35 $41^{\prime} \mathrm{N}, 8^{\circ} 47^{\prime} \mathrm{W}, 25-\mathrm{IV}-1928$, Ander son s.n. (MO): Campbell Co., Core Lake, Caryville, $36^{\circ} 18^{\prime} \mathrm{N}, 84^{\circ} 12^{\prime} \mathrm{W}, 1-\mathrm{V}-1959$. Comte 1023 (MO); Fentress
Co., Clear Creek ravine by US 127, $1.2 \mathrm{mi}$ S Clarkrange, $36^{\circ} 11{ }^{\prime} \mathrm{N}, 8^{\circ} 1^{\prime} \mathrm{W}, \mathrm{l}-\mathrm{V}-1971$, Kral 42384 (MO); Franklin Co., Cumberland mt. at Coven, $35^{\circ} 23^{\prime} \mathrm{N}, 85^{\circ} 52^{\prime} \mathrm{W}, 5-\mathrm{V}$ 1898, Eggert s.n. (MO); Grainger Co., near Fowlers, $36^{\circ} 16^{\circ} \mathrm{N}, 83^{\circ} 29^{\circ} \mathrm{W}, 9-\mathrm{V}-1934$, Cook s.n. (MO); Hardin Co. by Ten $69.21 \mathrm{mi}$ SE Savannah, $35^{\circ} 13^{\prime} \mathrm{N}, 8^{\circ} 14^{\prime} \mathrm{W}, 2$-V 1970, Kral 38929 (MO); Hickman Co., Bucksnort, 35 $53^{\prime} \mathrm{N}, 87^{\circ} 41^{\prime} \mathrm{W}, 21-\mathrm{IV}-1972$, Kral 45630 (MO); Knox Co., Knoxville, $35^{\circ} 57^{\prime} \mathrm{N}, 8^{\circ} 55^{\prime} \mathrm{W},-\mathrm{V}$-, Ruth 342 (MO); Lewis Co.. $3.1 \mathrm{mi}$ WSW Hampshire, $35^{\circ} 34^{\prime} \mathrm{N}, 87^{\circ} 20^{\prime} \mathrm{W}$, 1V-1970, Kral 38855 (MO); Macon Co., S Lafayette, $36^{\circ} 31^{\prime} \mathrm{N}, 86^{\circ} \mathrm{I}$ 'W, 5-V-1975, Kral 55215 (MO); Overton Co., along West Fork of Obey River by Tenn. 52, $3.4 \mathrm{mi} \mathrm{E}$ Alpine, $36^{\circ} 23^{\circ} \mathrm{N}, 85^{\circ} 13^{\prime} \mathrm{W}, 31-\mathrm{IV}-1971$. Kral 42317 (MO); Pickett Co., $1.5 \mathrm{mi}$ N of Pickett Park, $36^{\circ} 33^{\prime} \mathrm{N}, 84^{\circ} 48^{\prime} \mathrm{W}, 5-$ V-1973, Kral 49862 (MO). VERMONT: Bennington Co., Pownal, $42^{\circ} 45^{\circ} \mathrm{N}, 73^{\circ} 14^{\prime} \mathrm{W}, 23-\mathrm{V}-1962$, Richardson s.n. (MO); Chittenden Co., Burlington, $44^{\circ} 28^{\prime} \mathrm{N}, 73^{\circ} 12^{\prime} \mathrm{W}, 15$ VII-1939, E.J.W. 1170 (VT); Rutland Co., Brandon, $43^{\circ} 47^{\prime} \mathrm{N}, 73^{\circ} 5^{\prime} \mathrm{W}, 23-\mathrm{VI}-1927$, Dutton s.n. (VT); Whitingham, 42 47'N, 72 53'W, 26-VII-1915, Dutton s.n. (MO).

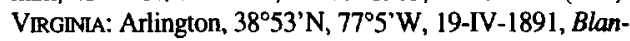
chard s.n. (MO); Bedford Co., Blue Ridge Pkwy MP 69, $37^{\circ} 18^{`} \mathrm{~N}, 79^{\circ} 34^{\prime} \mathrm{W}, 24-\mathrm{IV}-1984$, Guenther 47 (MO); Richmond, Westchester Park, $37^{\circ} 33^{\prime} \mathrm{N}, 77^{\circ} 27^{\prime} \mathrm{W}, 13-\mathrm{V}-1911$, Churchill s.n. (MO). West VIRGINIA: Mingo Co., near Varmy school, $37^{\circ} 42^{\prime}$ N, $82^{\circ} 9$ 'W 7 -VII-1930, Berkley 975 (MO); Raleigh Co., Rt. 412 mi W of Fayette Co., 37 $51^{\prime} \mathrm{N}$, 81 ${ }^{\circ}{ }^{\prime} \mathrm{W}, 14-\mathrm{IV}-1989$. Hill 20455 (MO); Upshur Co., near Buckhannon, 38 $59^{\prime} \mathrm{N}, 80^{\circ} 13^{\prime} \mathrm{W}, 10-\mathrm{V}-1895$, Pollock s.n.

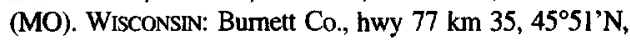
92 $23^{\prime}$ W, 20-VI-1970, Koch 6759 (NEB); Dane Co.. Utica, $42^{\circ} 57^{\prime}$ N, $89^{\circ} 7^{\prime}$ W, 12-V-1946, Fassett 26145 (OKL); Pierce Co., Beldenville, $44^{\circ} 46^{\circ}$ N, $92^{\circ} 30^{\prime} \mathrm{W}, 8-\mathrm{V}-1941$, Plett s.n. (MO); Racine Co., Caledonia, SW of Five Mile and Short Roads, $42^{\circ} 48^{\prime}$ N, $87^{\circ} 55^{\prime}$ W, 9-V-1976, Lorence 1689 (MO).

3. Geranium oreganum Howell, Fl. N. W. Amer. 1: 106 (1897)

G. canum Rydb. in Underw. \& Britton (eds.), N. Amer. Fl. 25(1): 14 (1907), nom. illeg.

Ind. loc.: "Edge of woods and open places throughout the Willamette valley"

Typus: USA. Oregon, Pacific Coast Plants, June 1881, Howell s.n. (lectotype, here designated, OSC!; isolectotype, LE!)

= G. albiflorum var. incisum Nutt. ex Torr. \& A. Gray, Fl. N. Amer. 1: 206 (1838)

G. incisum (Nutt. ex Torr. \& A. Gray) Howell, Fl. N. W. Amer. 1: 106 (1897), nom. illeg., non Andrews 1799

G. hookerianum var. incisum (Nutt. ex Torr. \& A. Gray) Walp., Repert. Bot. Syst. 1: 450 (1842)

Ind. loc.: "Vallies of the Rocky Mountains and Oregon, Nuttall!" 


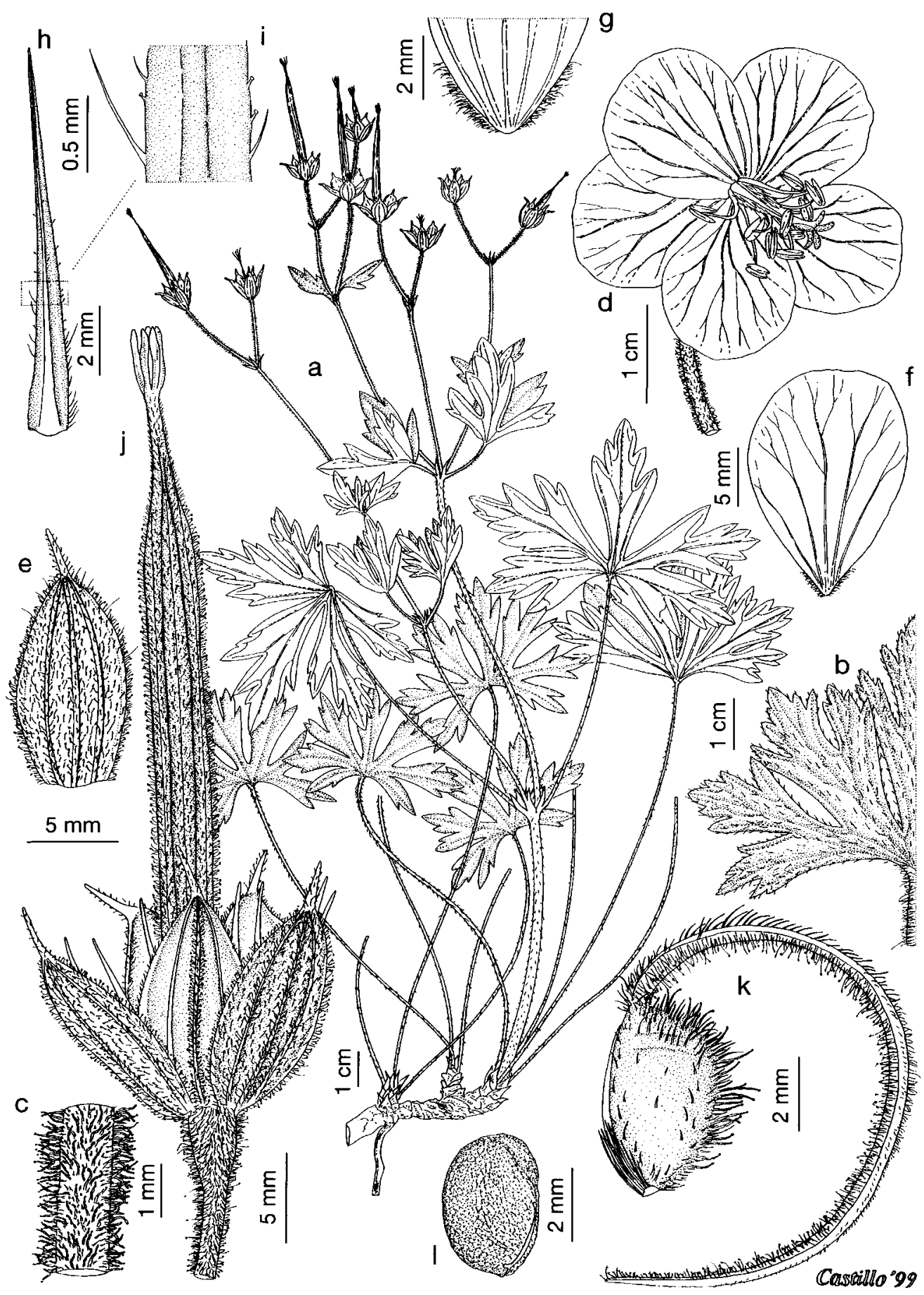

Fig. 7.-Geranium oreganum: a, Habit; b, Leaf; c, Peduncle; d, Flower; e, Sepal; f, Petal; g, Base of petal; h, i, Staminal filament; j, Fruit; k, Mericarp; 1, seed. [a-i based on Henderson 12973 (OSC), j-1 based on Leach 236 (OSC)]. 
Typus: USA. Rocky Mountains, Oregon, Nuttall s.n. (lectotype, here designated, BM!)

Herbs perennial, (15-)40-80 cm tall. Rootstock 5-6 mm diam., horizontal, not tuberculate, not turnip-shaped. Stem erect, solitary, with patent to retrorse, eglandular hairs 0.2-0.6 $\mathrm{mm}$ long. Basal leaves in a \pm deciduous rosette; lamina 6-12 $\times 7-14 \mathrm{~cm}$, polygonal in outline, cordate, palmatifid (divided for $0.78-0.9$ of its length), pilose, with appressed, eglandular hairs; segments 7, mombic, 5-7 $\mathrm{mm}$ at the base, 12-19-lobed in distal half (ratio main-sinus length of the middle segment / middle-segment length $=0.18-0.38$ ); cauline leaves opposite; petioles to $35 \mathrm{~cm}$ long, with patent to retrorse, eglandular hairs $0.2-0.6 \mathrm{~mm}$ long; stipules $10-$ $12 \times 2-3 \mathrm{~mm}$, lanceolate, with eglandular hairs on abaxial surface and on the margin, glabrous adaxially. Inflorescence a dichasial cyme; cymules 2-flowered, solitary or rarely in aggregates at the top of each branch; peduncles (0-) $4.5-7.7 \mathrm{~cm}$ long, with patent to retrorse, eglandular hairs $0.2-0.7 \mathrm{~mm}$ long, and patent, glandular hairs 0.3-1.1 mm long; bracteoles 4-7 $\times 1$ $\mathrm{mm}$, linear, with eglandular -and usually glandular hairs- on both sides and on the margin; pedicels $1-3.5 \mathrm{~cm}$ long, with patent to retrorse, eglandular hairs 0.2-0.7 mm long, and patent, glandular hairs 0.3-1.1 mm long; pedicel and peduncle together often overtopping the subtending leaf. Sepals 9-11 $\times$ 4-5 mm (ratio pedicel length / sepal length $=1-4$ ), smooth, not accrescent, 3-5-nerved, with mucro $1.5-2 \mathrm{~mm}$ long, with scarious margins $0.1-0.2 \mathrm{~mm}$ wide, with patent, eglandular hairs $0.2-0.6 \mathrm{~mm}$ long and patent, glandular hairs $0.3-0.7 \mathrm{~mm}$ long on the abaxial side, glabrous adaxially. Petals 13 $20 \times 12-16 \mathrm{~mm}$ (ratio petal length / petal width $=1.2-1.35$ ), erect-patent, entire or slightly notched (notch ca. $0.5 \mathrm{~mm}$ deep), without claw, glabrous on both sides, ciliate on the basal margin, purplish. Stamens 10 , both whorls bearing anthers; filaments $8-10 \mathrm{~mm}$ long, not exserted, lanceolate, pilose on the abaxial side, ciliate on the proximal half, with up to hairs $0.4 \mathrm{~mm}$ long; anthers $2.5-3 \times 1.5$ $\mathrm{mm}$. Nectaries with a tuft of hairs at the top, dorsally glabrous. Gynoecium 8-10 mm long.
Fruit 35-52 mm long, erect when immature; mericarps $4.5-5 \times 2.5-3 \mathrm{~mm}$, without a strand of fibres, smooth, without longitudinal rib, without basal beak, with a basal callus, with \pm patent, eglandular hairs $0.1-0.3 \mathrm{~mm}$ long, and glandular $0.3-0.8 \mathrm{~mm}$ long, brownish; rostrum $27-44 \mathrm{~mm}$ long, with a narrowed apex 4-6 $\mathrm{mm}$ long, with patent, glandular hairs $0.3-0.8 \mathrm{~mm}$ long and patent, eglandular hairs $0.1-0.4 \mathrm{~mm}$ long; stigmatic remains 3-6 mm long (ratio fruit length / stigmatic remains length $=6-11$ ), with 5 glabrous lobes. Seeds $2.8-3 \times 2-2.1 \mathrm{~mm}$, reticulate, brownish; hilum $1 / 4$ as long as the perimeter. Cotyledons entire.

Flowering March-July. Open woods, moist meadows or roadsides; $50-1500 \mathrm{~m}$. Northwestern U.S.A. Fig. 9.

\section{Illustrations. Fig. 7, 8b.}

Geranium oreganum is easily distinguished by the combination of the following three characters: long petals glabrous on the adaxial surface, pedicels with glandular hairs, and nectaries with a tuft of hairs at the top. Differences between $G$. oreganum and $G$. maculatum are addressed in the discussion of the latter species. In $G$. oreganum glandular hairs are always present on inflorescence, sepals and mericarps. They are similar to glandular hairs of $G$. richardsonii or G. viscosissimum: shorter than in G. maculatum and ending in a broad usually red cell.

According to Scoggan (1978: 1046) G. oreganum grows in British Columbia and Alberta. These records seem to be based on Dawson 890 (CAN), and Macoun 10078 (CAN) respectively, which are $G$. viscossisimum. Additionally, $G$. oreganum is not mentioned in the Flora of Alberta (PACKER, 1983), and no other specimen supporting its presence in Alberta has been found. According to DouGLAS \& al. (1989: 51) G. oreganum is known in British Columbia from a single collection at Victoria. I have studied this flowering collection [Anderson 200 (V)], which is G. ibericum Cav. This specimen, collected in 1896, seems to be an occasional garden-escape probably not established. 


\section{Representative specimens examined}

USA. CALIFORNIA: Humboldt Co., Hubbard's Station, $40^{\circ} 12^{\prime} \mathrm{N}, 123^{\circ} 44^{\prime} \mathrm{W}, 10-\mathrm{VI}-1899$, Burtt Davy 5393 (UC); Humboldt Co., Middle Yager Creek, $40^{\circ} 34^{\prime} \mathrm{N}$, 123⒌'W, 18-VI-1922, Tracy 6094 (UC); Humboldt Co., Trinity River, near Willow Creek, $40^{\circ} 56^{\prime} \mathrm{N}$, $123^{\circ} 37^{\prime}$ W, 4-VII-1911, Tracy 3287 (UC); Humboldt Co., Trinity River, South Fork, $40^{\circ} 20^{\prime} \mathrm{N}, 123^{\circ} 54^{\prime} \mathrm{W}, 27$ IV-1924, Tracy 6663 (UC); Siskiyou Co., Hupa Indian Reservation, $41^{\circ} 4^{\prime} \mathrm{N}, 123^{\circ} 48^{\prime} \mathrm{W}$, -VI-, Chandler 1272 (UC); Siskiyou Co., Mount Shasta, Sisson, $41^{\circ} 24$ 'N, $122^{\circ} 12^{\prime} \mathrm{W}, 13-\mathrm{VII}-1902$, Setchell s.n. (UC); Siskiyou mountains, $41^{\circ} 50^{\prime} \mathrm{N}, 123^{\circ} 40^{\prime} \mathrm{W}$, -VII-, Brandegee s.n. (UC). OREGON: Benton Co., $44^{\circ} 29^{\prime} \mathrm{N}, 123^{\circ} 25^{\prime} \mathrm{W}$, -VI-,
Standlee s.n. (OSC); Benton Co., Corvallis vicinity, MacDonald Forest, $44^{\circ} 33^{\prime} \mathrm{N}, 123^{\circ} 15^{\prime} \mathrm{W}, 3-\mathrm{VI}-1989$, Chambers 5455 (OSC); Benton Co., NE Corvallis, west of Canterbury Circle, area around Frazier Creek, $44^{\circ} 36^{\prime} \mathrm{N}$, $123^{\circ} 19^{\prime} \mathrm{W}, 18-\mathrm{V}-1980$, Halse 2178 (OSC); Benton Co., SW of Corvallis, $44^{\circ} 35^{\prime} \mathrm{N}, 123^{\circ} 16^{\prime} \mathrm{W}, 16-\mathrm{V}-1999$, Halse 5533 (MA); Clackamas Co., Estacada, $45^{\circ} 17^{\prime} \mathrm{N}$, $122^{\circ} 19^{\prime} \mathrm{W}, 2-\mathrm{VII}-1905$, Lyon 112 (F); Douglas Co., Canyonville, $42^{\circ} 55^{\prime} \mathrm{N}, 123^{\circ} 16^{\prime} \mathrm{W}, 7-\mathrm{V}-1940$, Gould 1089 (UC); Douglas Co., Thicket $3 \mathrm{mi}$ W Coos junction, $43^{\circ} 25^{\prime} \mathrm{N}, 124^{\circ} 13^{\prime} \mathrm{W}, 27-\mathrm{VI}-1939$, Peck 20277 (OSC); Jackson Co., $29 \mathrm{mi}$ S of Prospect, $42^{\circ} 45^{\prime} \mathrm{N}, 122^{\circ} 29^{\prime} \mathrm{W}, 3-$ VII-1936, Thompson 13087 (MO); Jackson Co., Cascade Mountains, Jenny Creek, $41^{\circ} 58^{\prime} \mathrm{N}, 122^{\circ} 24^{\prime} \mathrm{W}, 21-\mathrm{VI}-$
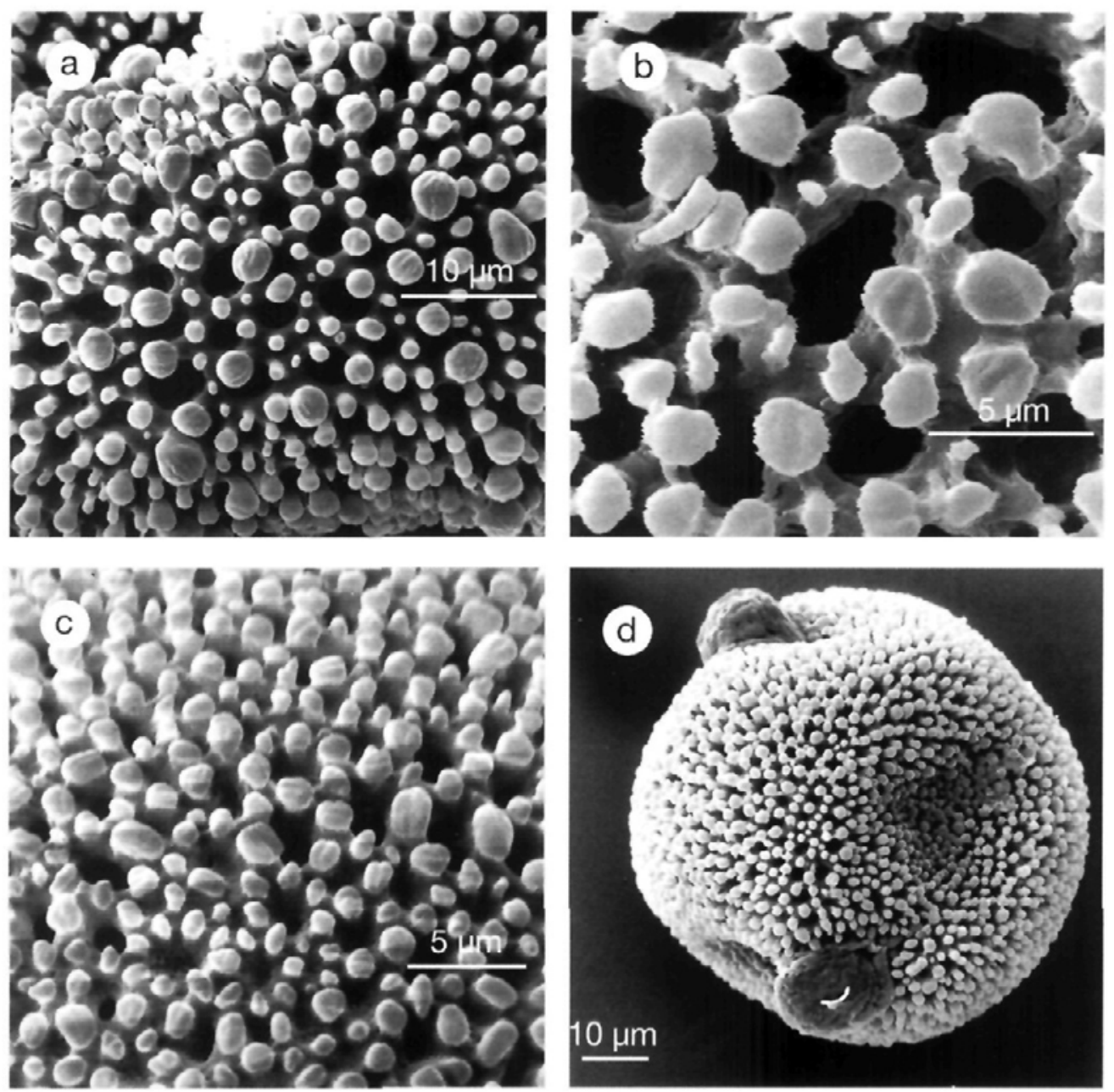

Fig. 8.-SEM photomicrographs showing pollen of some perennial Geranium native from North America. As in the almost entire genus it is characterised by reticulate exine ornamentation with distinctly baculate, clavate, or gemmate supratectal elements: a, G. erianthum [Dempster 1335 (UC)]; b, G. oreganum [Hammond 63 (UC)]; c, G. lentum [Lehto \& al. $11564(\mathrm{NY})] ; \mathrm{d}, \mathrm{G}$. wislizenii [Mayfield 179 (TEX)]. 


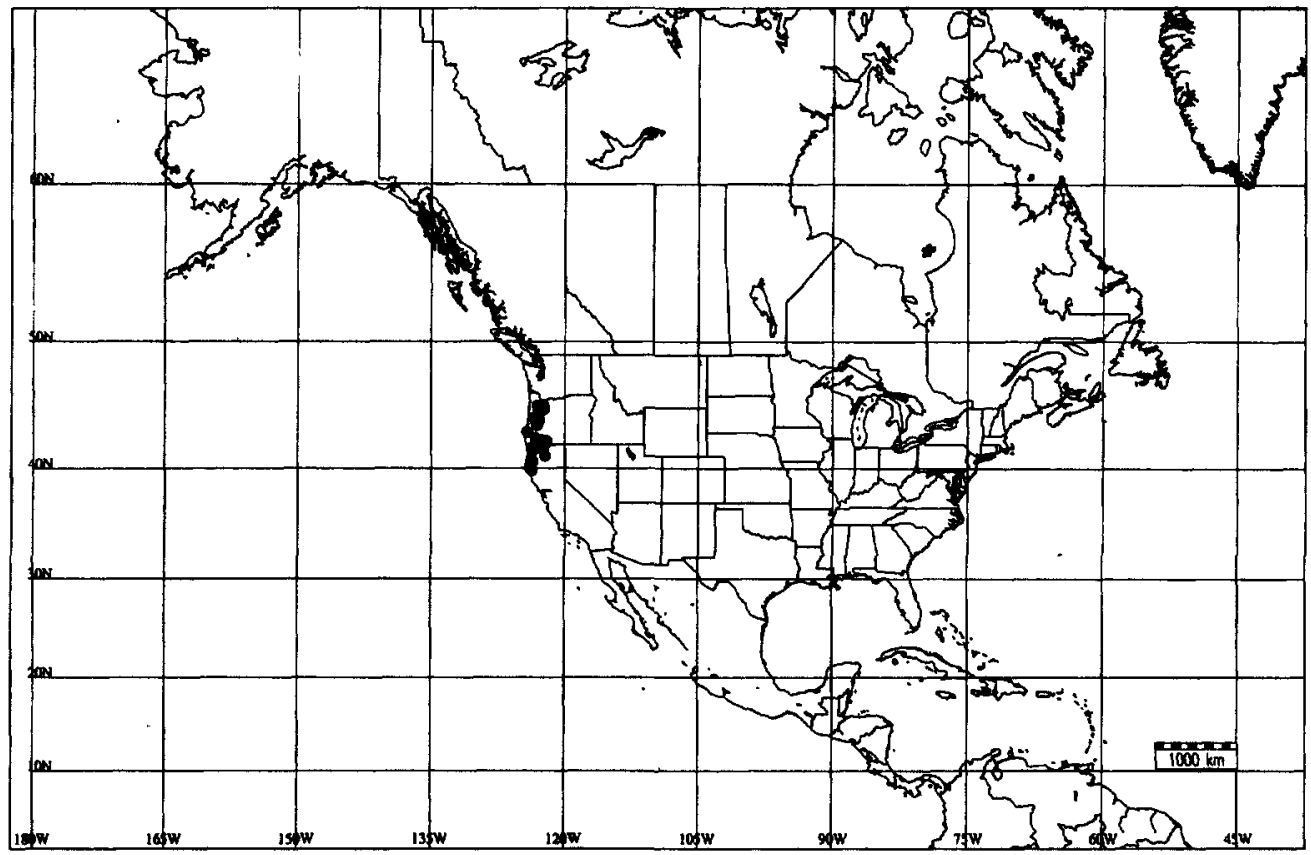

Fig. 9.-Distribution of Geranium oreganum.

1925, Applegate 4394 (OSC); Jackson Co., Elk Creek into Rogue River, $42^{\circ} 39^{\circ} \mathrm{N}, 122^{\circ} 45^{\prime} \mathrm{W}, 10-\mathrm{VI}-1930$, Henderson 12973 (OSC); Jackson Co., Grizzly Peak, $42^{\circ} 16^{\prime} \mathrm{N}, 122^{\circ} 37^{\prime} \mathrm{W}, 21$-VI-1925, Applegate 4394 (OSC); Jackson Co., Lily Glen Campground at Howard Prairie Lake on Dead Indian Rd., $42^{\circ} 13^{\prime} \mathrm{N}, 122^{\circ} 22^{\prime} \mathrm{W}$, 20-VII-1975, Crosby 316 (OSC); Jackson Co., N of Siskiyou Inn., $42^{\circ} 4^{\prime} \mathrm{N}, 122^{\circ} 36^{\prime} \mathrm{W}, 13-\mathrm{VI}-1930$, Henderson 12975 (OSC); Jackson Co., Trail, 42 ${ }^{\circ} 40^{\prime} \mathrm{N}$, $122^{\circ} 50^{\prime} \mathrm{W}, 3-\mathrm{VI}-1924$, Shevwood 806 (OSC); Jackson Co., Wimer, $42^{\circ} 32^{\prime} \mathrm{N}, 123^{\circ} 8^{\prime} \mathrm{W}, 23-\mathrm{V}-1893$, Hammond 63 (UC); Klamath Co., $8 \mathrm{mi}$ W of Lake of the Woods, $42^{\circ} 22^{\prime}$ N, $122^{\circ} 14^{\prime} \mathrm{W}, 5$-VII-1931, Peck 16672 (OSC); Klamath Co., Fort Klamath, $42^{\circ} 42^{\prime} \mathrm{N}, 121^{\circ} 59^{\prime} \mathrm{W}, 17-$ VII-1970, Peck 9560 (MO); Klamath Co., Jack Springs, $42^{\circ} 37^{\prime} \mathrm{N}, 122^{\circ} 4^{\prime} \mathrm{W}, 16-\mathrm{VI}-1931$, Evans 219 (OSC); Lane $\mathrm{Co}$., at junction of Lorane Highway and Cret Drive, $3 \mathrm{mi}$ SW of Eugene, $44^{\circ} 3^{\prime} \mathrm{N}, 123^{\circ} 5^{\prime} \mathrm{W}, 5-\mathrm{VII}-1934$, Constance 941 (MO); Lane Co., east of Eugene, $44^{\circ} 3^{\prime} \mathrm{N}$, $123^{\circ} 5^{\prime}$ W, 10-III-1934, Ontrank s.n. (OSC); Lane Co., Eugene, $44^{\circ} 3^{\prime} \mathrm{N}, 123^{\circ} 5^{\prime} \mathrm{W}, 27-\mathrm{VI}-1927$, Leach 236 (OSC); Lane Co., Spencers Butte, $43^{\circ} 58^{\prime} \mathrm{N}, 123^{\circ} 8^{\prime} \mathrm{W}$, 24-VI-1930, Henderson 1270 (OSC); Linn Co., $2 \mathrm{mi} \mathrm{NW}$ Peterson Butte, $44^{\circ} 30^{\prime} \mathrm{N}, 122^{\circ} 58^{\prime} \mathrm{W}, 23-\mathrm{V}-1966$, Hitchcock 24403 (UC); Linn Co., Crawfordsville, $44^{\circ} 21^{\prime} \mathrm{N}$, $122^{\circ} 51^{\prime}$ W, 14-VI-1931, Henderson 13642 (OSC); Marion Co., Salem, $44^{\circ} 55^{\prime} \mathrm{N}, 123^{\circ} 1^{\prime} \mathrm{W}$, Reynolds 8972 (OSC); Marion Co., Salem, $44^{\circ} 55^{\prime} \mathrm{N}, 123^{\circ} 1^{\prime} \mathrm{W}, 14-\mathrm{VI}-$ 1909, Peck 4770 (OSC); Marion Co., Silver Creek, $45^{\circ} 0^{\prime} \mathrm{N}, 122^{\circ} 50^{\prime} \mathrm{W},-1871$, Hall 73 (F); Multnomah Co., Eln Island near Portland, Lake Bene Mts, $45^{\circ} 30^{\prime} \mathrm{N}$, $122^{\circ} 35^{\prime} \mathrm{W}, 12-\mathrm{VI}-1886$, Henderson 158 (MO); Washington Co., Beaverton, $45^{\circ} 29^{\prime} \mathrm{N}, 122^{\circ} 48^{\prime} \mathrm{W}, 15-\mathrm{V}-1916$, Sisters of St Mary s.n. (UC); Washington Co., near Aloha, $45^{\circ} 29^{\prime} \mathrm{N}, 122^{\circ} 51^{\prime} \mathrm{W}, 26-\mathrm{V}-1928$, Thompson 4275 (MO); Washington Co., near Forest Grove, $45^{\circ} 31^{\prime} \mathrm{N}$, $123^{\circ} 6^{\prime} \mathrm{W}, 10-\mathrm{V}-1926$. Thompson 584 (MO); Washington Co., Sherwood, $45^{\circ} 21^{\prime}$ N, $122^{\circ} 50^{\prime} \mathrm{W}, 29-\mathrm{V}-1951$, Hitchcock 19283 (UC). Washington: Clark Co., Lacamas Creck, $45^{\circ} 35^{\prime} \mathrm{N}, 122^{\circ} 23^{\prime} \mathrm{W}, 1936$, Jones s.n. (ILL).

\section{Geranium sylvaticum L., Sp. Pl.: 681} (1753)

Ind. loc.: "Habitat in Europae borealis sylvis"

Typus.: "Habitat in Europae borealis sylvis" [lectotype, designated by Yeo in JARVIS \& AL. (1993: 49), LINN 858.63 colour slide!]

Herbs perennial, $15-70 \mathrm{~cm}$ tall. Rootstock 8-10 mm diam., \pm horizontal, not tuberculate, not turnip-shaped. Stem erect, solitary, with patent, eglandular and glandular hairs 0.7-1.2 $\mathrm{mm}$ long (glandular hairs mainly on the upper part). Basal leaves in a deciduous rosette; lamina 6-12 $\times 8-16 \mathrm{~cm}$, polygonal in outline, cordate, palmatifid (divided for $0.76-0.85$ of its 
length), pilose, with appressed, eglandular hairs; segments 5-7, rhombic, $6-12 \mathrm{~mm}$ at the base, 24-34-lobed in distal half (ratio main-sinus length of the middle segment $/$ middlesegment length $=0.16-0.22$ ); cauline leaves alternate (upper usually opposite); petioles to $20 \mathrm{~cm}$ long, with patent, eglandular hairs $0.7-1$ mm long; stipules 9-15 × 1-2 mm, lanceolate, with eglandular hairs on abaxial surface and on the margin, usually glabrous adaxially. Inflorescence a dichasial cyme; cymules 2 -flowered, solitary or in aggregates at the top of each branch: peduncles $2-7 \mathrm{~cm}$ long, with patent, glandular and eglandular hairs $0.5-1.1 \mathrm{~mm}$ long; bracteoles 4-6 $\times 1 \mathrm{~mm}$, lanceolate, with eglandular hairs on abaxial surface and on the margin, usually glabrous adaxially; pedicels $0.8-1.5 \mathrm{~cm}$ long, with patent, glandular and eglandular hairs $0.5-1.1 \mathrm{~mm}$ long; pedicel and peduncle together overtopping the subtending leaf. Sepals 8-9 $\times 3-4 \mathrm{~mm}$ (ratio pedicel length / sepal length $=0.9-2$ ), smooth, not accrescent, 3-5-nerved, with mucro 2-3.2 mm long, with scarious margins $0.5-1 \mathrm{~mm}$ wide, with \pm patent, eglandular and glandular hairs 0.5-0.9 $\mathrm{mm}$ long. Petals $18-21 \times 12-13 \mathrm{~mm}$ (ratio petal length / petal width $=1.4-2.1$, erectpatent, entire or slightly notched (notch ca. 1 $\mathrm{mm}$ deep), without claw, hairy on the base of adaxial side, glabrous on the abaxial side, ciliate on the basal margin, purplish. Stamens 10, both whorls bearing anthers; filaments 9-11 $\mathrm{mm}$ long, not exserted, lanceolate, pilose on the abaxial side, ciliate on the proximal half, with hairs $0.2-0.7 \mathrm{~mm}$ long; anthers $2-2.3 \times 1$ $1.1 \mathrm{~mm}$. Nectaries glabrous. Gynoecium 10$11 \mathrm{~mm}$ long. Fruit 28-35 mm long, erect when immature; mericarps 4.5-5.2 $\times 2-2.5 \mathrm{~mm}$, without a strand of fibres, smooth (with 2 transversal veins at the apex), without longitudinal rib, without basal beak, with a basal callus, with \pm appressed, eglandular hairs 0.2-0.6 $\mathrm{mm}$ long, and glandular $0.7-0.9 \mathrm{~mm}$ long, brownish; rostrum $22-27 \mathrm{~mm}$ long, with a narrowed apex 3-4 mm long, with \pm patent, eglandular hairs $0.2-0.5 \mathrm{~mm}$ long, and glandular $0.8-1 \mathrm{~mm}$ long; stigmatic remains $2.2-3 \mathrm{~mm}$ long (ratio fruit length / stigmatic remains

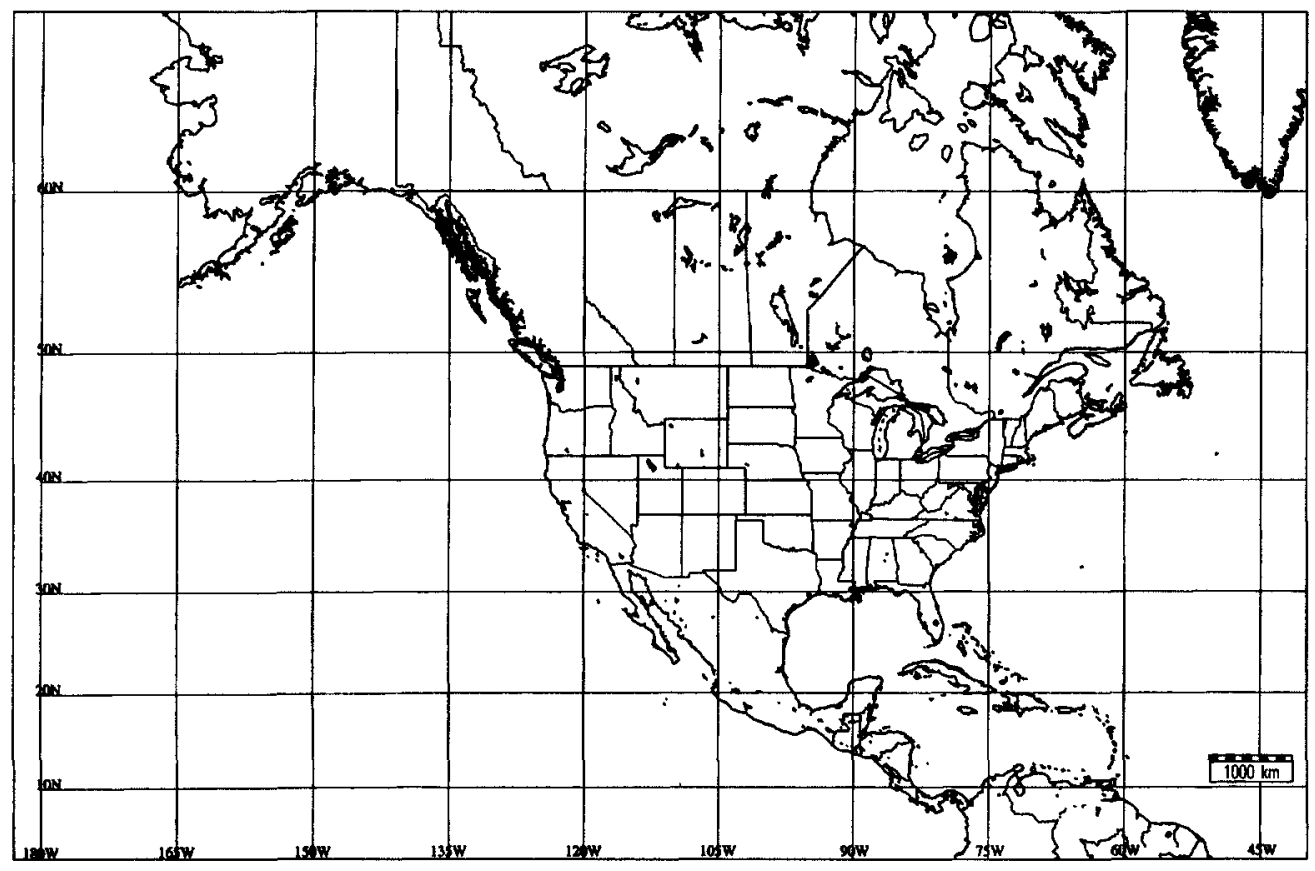

Fig. 10.-Distribution of Geranium sylvaticum in North America. 
length $=10.6-15.5$ ), with 5 glabrous lobes. Seeds $2.9-3.1 \times 1.8-2 \mathrm{~mm}$, finely reticulate, reddish; hilum $1 / 4$ as long as the perimeter. Cotyledons entire. $2 n=28, n=14$.

Flowering July-August. Habitat and altitude unknown in Greenland. Europe, Caucasus, Siberia, Western Asia \& Subartic America. Fig. 10.

Illustrations. Fig. 11. Ross-CraIG (1952, pl. 31); TOKARSKI (1972: 71, pl. 37); YEO (1992a: 72 fig. 9.9).

Geranium sylvaticum can be distinguished from other native North American species with larger and glabrous (abaxially) petals by its glabrous nectaries, pedicels densely glandular-hairy, and staminal filaments with short hairs. Geranium sylvaticum might at first be mistaken for Geranium pratense, but the former has immature erect fruit, glabrous nectaries and staminal filaments without an abruptly narrowed apex. It is a widespread species in Europe, occurring in North America only on Greenland coast.

According to RouSSEAU (1968: 103) in Quebec this species was cultivated, and occasionally garden-escape but probably not naturalished.

\section{Representative specimens examined}

GreENLAND. Tugtutôq, $60^{\circ} 48^{\prime} \mathrm{N}, 46^{\circ} 30^{\circ} \mathrm{W}, 29$-VII1963, Hansen \& Jakobsen 213 (BM); Kangikitsoq, Tunerqat, $60^{\circ} 16^{\prime} \mathrm{N}, 44^{\circ} 14^{\prime} \mathrm{W}, 15$-VIII-1966, Hansen 440 (BM): Kangerssuneq qingordleq. Ánivia, $60^{\circ} 19^{\circ} \mathrm{N}$, 44ㄱ' W, 8-VII-1966, Hansen 66-1089 (MO).

5. Geranium pratense L., Sp. Pl.: 681 (1753)

Ind. loc.: "Habitat in Europae borealis pratis"

Typus: "Habitat in Europae borealis pratis" (lectotype, here designated, LINN 858.66, slide colour!) [choice made by $B$. Jonsell, and C. Jarvis]

Herbs perennial, $40-100 \mathrm{~cm}$ tall. Rootstock 12-20 mm diam., \pm horizontal, not tuberculate, not turnip-shaped. Stem erect, usually few per caudex branch, with retrorse, \pm appressed, eglandular hairs 0.1-0.5 mm long, and patent, glandular hairs $0.2-0.9 \mathrm{~mm}$ long mainly on the upper part. Basal leaves in a deciduous rosette; lamina 6.5-9 × 8-16 cm, polygonal in outline, cordate, palmatifid (divided for $0.85-0.93$ of its length), pilose, with appressed, eglandular hairs; segments 5-7, rhombic, $3-5 \mathrm{~mm}$ at the base, 10-30-lobed in distal half (ratio main-sinus length of the middle segment / middle-segment length $=0.15-0.48$ ); cauline leaves opposite; petioles to $30 \mathrm{~cm}$ long, with retrorse, appressed, eglandular hairs ca. $0.4 \mathrm{~mm}$ long; stipules 11-16 × 3-4 mm, lanceolate, with eglandular hairs on abaxial surface and on the margin, usually glabrous adaxially. Inflorescence a dichasial cyme; cymules 2-flowered, solitary; peduncles $4.5-12 \mathrm{~cm}$ long, with patent, glandular hairs $0.6-0.8 \mathrm{~mm}$ long and retrorse, eglandular hairs 0.2-0.4 mm long; bracteoles 4$11 \times 1-3 \mathrm{~mm}$, lanceolate, with eglandular hairs on both sides and on the margin; pedicels 1.5 $2.5 \mathrm{~cm}$ long, with patent, glandular hairs $0.6-0.8$ $\mathrm{mm}$ long and \pm patent, eglandular hairs 0.2-0.4 $\mathrm{mm}$ long; pedicel and peduncle together overtopping the subtending leaf. Sepals 11-12 $\times 4$ $4.5 \mathrm{~mm}$ (ratio pedicel length $/$ sepal length $=1$ 3.1), smooth, not accrescent, 3-5-nerved, with mucro 2-3.5 mm long, with scarious margins 0.1-0.4 mm wide, with \pm patent eglandular and glandular hairs $0.5-0.9 \mathrm{~mm}$ long. Petals $18-20 \times$ $12-13 \mathrm{~mm}$ (ratio petal length $/$ petal width $=1.5$ 2.1), erect-patent, entire, without claw, glabrous on both sides, ciliate on the basal margin, bluish. Stamens 10, both whorls bearing anthers; filaments 7-9 mm long, not exserted, lanceolate with an abruptly narrowed apex, glabrous on both sides, ciliate on the proximal third, with hairs 0.2-0.6 mm long; anthers 2-2.5 $\times 0.8-1 \mathrm{~mm}$. Nectaries with a tuft of hairs at the top, dorsally glabrous. Gynoecium $9-12 \mathrm{~mm}$ long. Fruit 34-36 mm long, down-wardly inclined or reflexed when immature; mericarps 4 $6 \times 2.5-3 \mathrm{~mm}$, without a strand of fibres, smooth (sometimes with 1-2 transversal veins at the apex), without longitudinal rib, without basal beak, with a basal callus, with \pm appressed, eglandular and glandular hairs 0.2-0.5 $\mathrm{mm}$ long, brownish; rostrum 21-29 mm long, with a narrowed apex 7-8 mm long, with eglandular and glandular, \pm appressed hairs 0.2-0.6 $\mathrm{mm}$ long; stigmatic remains $2-3 \mathrm{~mm}$ long (ratio 


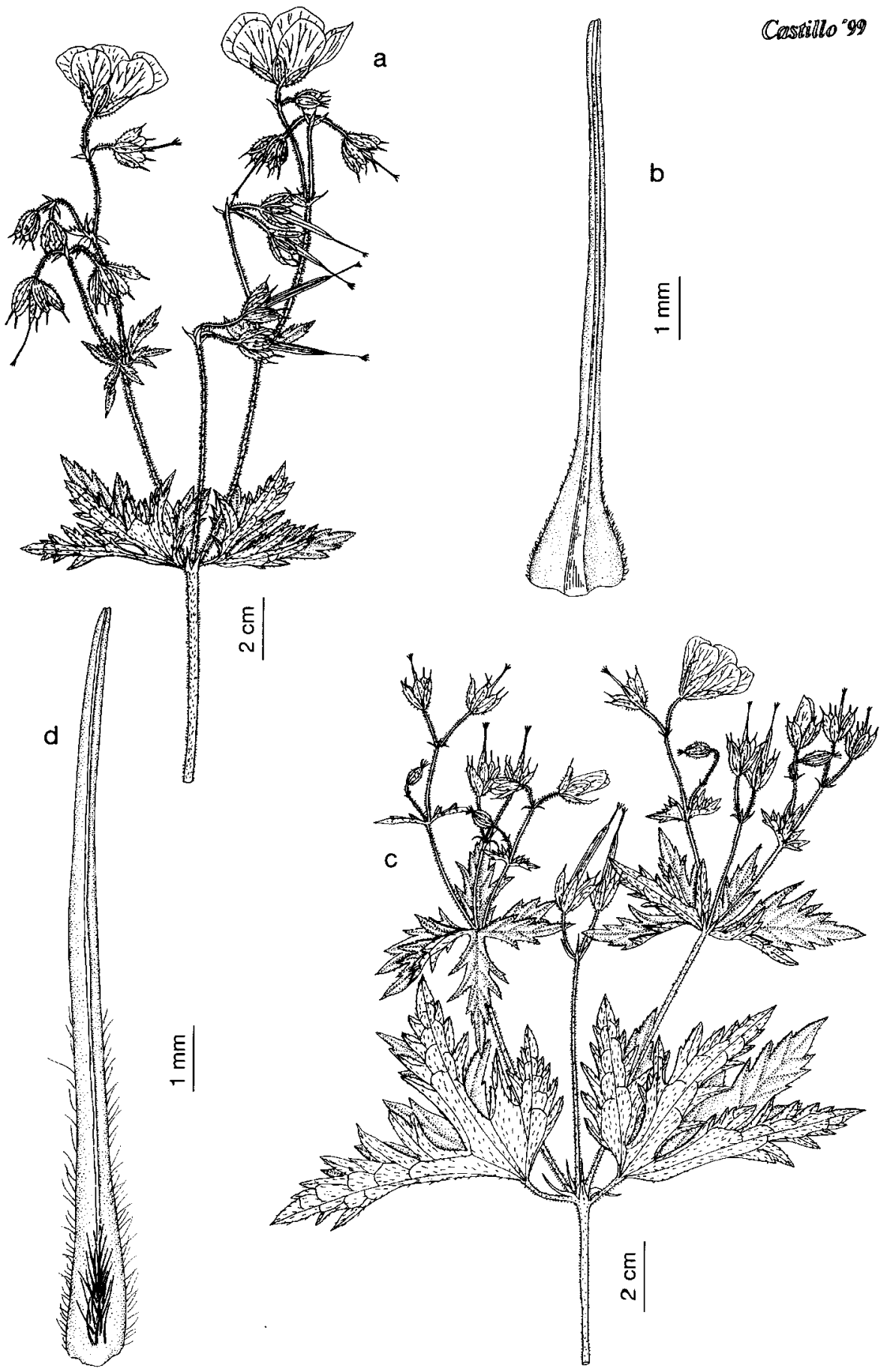

Fig. 11--Geranium pratense: a, Flowering and fruiting branch; b, Staminal filament [Aedo 2381 (MA)]. G. sylvaticum: c, Flowering and fruiting branch; d, Staminal filament [Aedo 2599 (MA)]. 
fruit length / stigmatic remains length $=11.7$ 16.6), with 5 glabrous lobes. Seeds 3-3.5 $\times 2$ $\mathrm{mm}$, reticulate, reddish; hilum $1 / 3$ as long as the perimeter. Cotyledons entire. $2 n=28,28+B$, $n=14$.

Flowering June-August. Meadows, waste grounds and roadsides; $0-1000 \mathrm{~m}$. Europe, Caucasus, Siberia, \& China; introduced from the Old World in Western Canada, Eastern Canada, and Northeastern U.S.A. Fig. 12.

Illustrations. Fig. 11. CAVANILles (1787, tab. 87 fig. 1); TOKARSKI (1972: 67, pl. 26); YEO (1992a: 81 fig. 9.17).

This introduced species also belongs to a group with large petals, glabrous on adaxial surface. However, G. pratense exhibits a unique feature among these species: downwardly inclined or reflexed fruit when immature. Additionally, G. pratense has staminal filaments with an abruptly narrowed apex, only occurring in $G$. erianthum.

According to RousSEAU (1968: 103) this species would have been naturalised in Cana- da since 1878. The specimens supporting the records of $G$. pratense from Manitoba (SCOGGAN 1978: 1044), and Pennsylvania (RHOADS \& KLEIN 1993: 258) have not been studied.

\section{Representative specimens examined}

CANADA. ALBERTA: along Atabasca River, Jasper Park, $52^{\circ} 53^{\prime} \mathrm{N}, 118^{\circ} 5^{\prime} \mathrm{W}, 25-\mathrm{VII}-1948$, Halpin 29 (DAO). LABRADOR: North West River, 5332' N, 60'9'W, 30-VII1950, Gillet \& Findlay 5528 (DAO GH, NY). New BRUNSWICK: Queens Co., Petersville, $45^{\circ} 30^{\prime} \mathrm{N}, 66^{\circ} 25^{\prime} \mathrm{W}$, 26-VI-1964, Squires s.n. (DAO); York Co., Maryland, 45 $56^{\prime}$ N $, 66^{\circ} 40^{\prime} \mathrm{W}, 2$-VII-1963, Roberts \& Drudy 63-377 (DAO); York Co.. Keswick Island, 45 $59^{\prime}$ N, $66^{\circ} 49^{\prime} \mathrm{W}$, 19-VI-1964. Squires s.n. (DAO). NEWFOUNDLAND I: Curl-

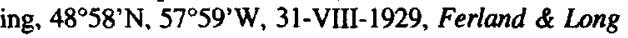
$1848(\mathrm{GH})$; Barred Island to Fogo Island, $50^{\circ} 43^{\prime} \mathrm{N}$, $56^{\circ} 6^{\prime} \mathrm{W}, 17-\mathrm{VIII}-1903$, Sornborger s.n. (GH); St. John's, $47^{\circ} 34^{\prime} \mathrm{N}, 52^{\circ} 34^{\prime} \mathrm{W}, 12-\mathrm{VII}-1937$, Brooks s.n. (MO); Avalon, Conception Bay, Salmon Cove, $47^{\circ} 30^{\prime} \mathrm{N}$, $53^{\circ} 30^{\prime} \mathrm{W}, 30-\mathrm{VII}-1982$, Crow \& al. 82-744 (DAO). Nova Scotia: Pictou Co., Springville, $45^{\circ} 27^{\prime} \mathrm{N}, 62^{\circ} 38^{\prime} \mathrm{W}, 7$ VI-1928, Saint John 1431 (NY); Yarmouth Co., Yarmouth, $43^{\circ} 50^{\prime} \mathrm{N}, 66^{\circ} 7^{\circ} \mathrm{W}$. 19-VIII-1920, Bissell \& Graves 21739 (PH GH); Washington Co., Grand Manan Island, $44^{\circ} 41^{\prime} \mathrm{N}, 66^{\circ} 49^{\prime} \mathrm{W}, 26-\mathrm{VII}-1939$, True $6958(\mathrm{PH})$. ONTARIO: Carleton Co., on Merivale road, $1 \mathrm{mi}$. from City View, $45^{\circ} 21^{\prime} \mathrm{N}, 7^{\circ} 45^{\prime} \mathrm{W}, 3-\mathrm{VII}-1948$, Anderson s.n.

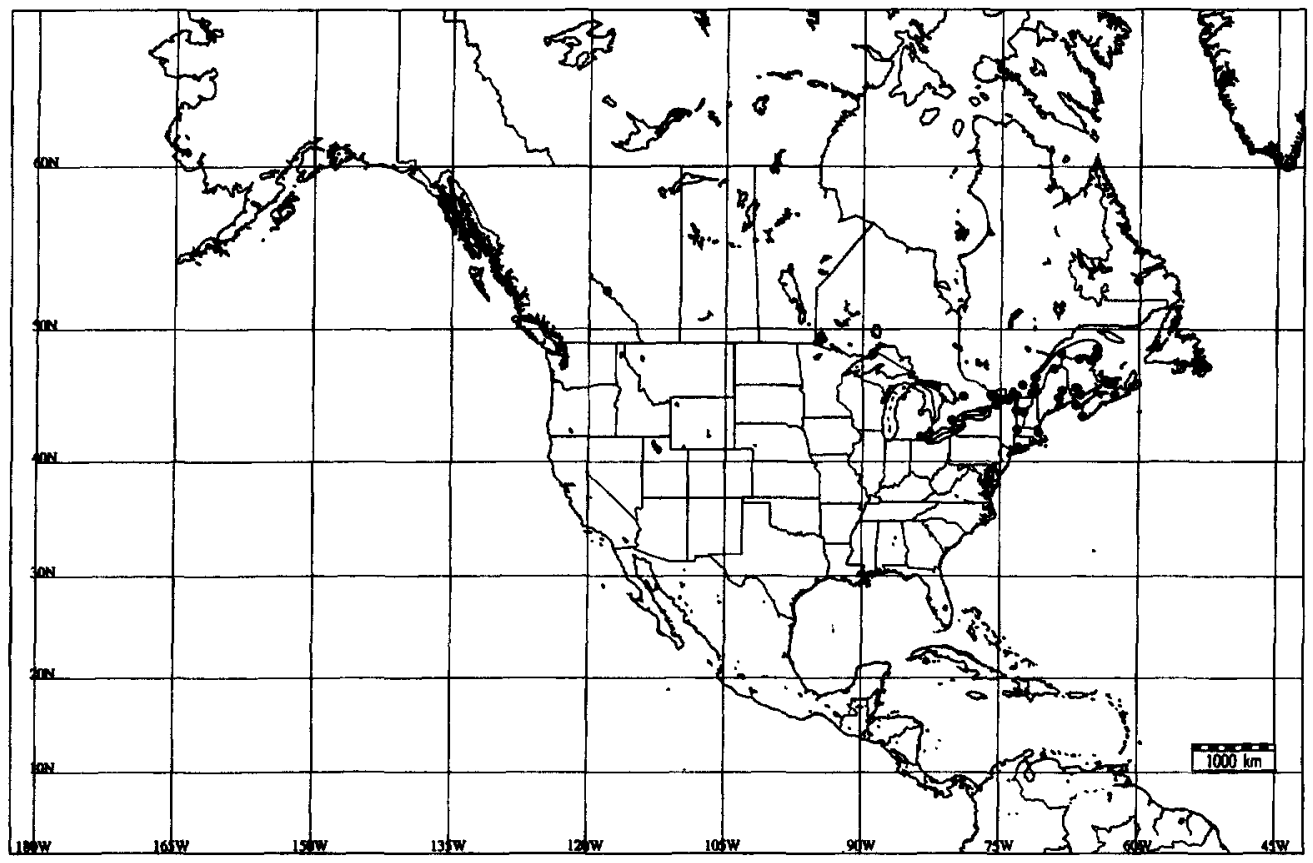

Fig. 12.-Distribution of Geranium pratense in North America. 
(DAO); Carleton Co., Carp airport, $45^{\circ} 21^{\prime} \mathrm{N}, 76^{\circ} 2^{\prime} \mathrm{W}, 13$ VII-1947, Calder \& Cody 1020 (DAO); Grenville Co., Prescott Highway, $44^{\circ} 43^{\prime} \mathrm{N}, 75^{\circ} 31^{\prime} \mathrm{W}, 11$-VII-1950, Bragg \& Basset 125 (DAO); Muskoka Co., 12 mi NE of Dwight at W gate of Algonquin Park, $45^{\circ} 20^{\prime} \mathrm{N}, 79^{\circ} 1^{\prime} \mathrm{W}$, 11-VII-1950, Bragg \& Basset 125 (DAO); Stormont Co., 2 mi south of Newington, Osanbruck Township, $45^{\circ} 7$ 'N, $75^{\circ} 1^{\prime} \mathrm{W}, 25-\mathrm{VII}-1955$, Jenkins 5751 (DAO); Wellington Co., Guelph Goldie's, 433' N, 80'15'W, 4-VIII-1914 (DAO). PrInce Edward I: Queens Co., Brackley Point, Blythe Hurst's, $46^{\circ} 26^{\prime} \mathrm{N}, 63^{\circ} 14^{\prime} \mathrm{W}, 22$-VIII-1953, Erskine \& Smith 2441 (DAO). QUÉBEC: Sillery, Anse-auxFoulons, 46 $46^{\prime} \mathrm{N}, 71^{\circ} 15^{\prime} \mathrm{W}, 16-\mathrm{VII}-1956$, Desmarcus 1719 (DAO); Ste. Adelaide, $48^{\circ} 50^{\prime} \mathrm{N}, 64^{\circ} 30^{\prime} \mathrm{W}, 12$-VIII1933, Théodule Proulx s.n. (MA); Sully, 47²7'N, 69'9'W, 10-VIII-1936, Ducharme 788 (DAO); Baie de Gaspé, rivière Dartmouth, $48^{\circ} 50^{\prime} \mathrm{N}, 64^{\circ} 29^{\prime} \mathrm{W}, 27-\mathrm{VII}-$ 1923. Marie-Victorin \& al. 17325 (DAO, MO); Gatineau Co., Eardley Parish, Breckenridge, $45^{\circ} 29^{\prime} \mathrm{N}, 75^{\circ} 57^{\prime} \mathrm{W}, 9$ VII-1948, Dore \& Boivin 8608 (DAO); Ile Ste-Helene, près de Montreal, 45 $30^{\prime} \mathrm{N}, 7^{\circ} 36^{\prime} \mathrm{W}, 25-\mathrm{VI}-1935$, Roy

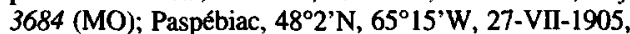
Churchill s.n. (MO); Chambly, $45^{\circ} 27^{\prime} N, 7^{\circ} 17^{\prime} \mathrm{W}, 18-$ VII-1938, Cléonique 10267 (DAO); Montreal, Ville Saint-Laurent, $45^{\circ} 30^{\prime} \mathrm{N}, 73^{\circ} 40^{\prime} \mathrm{W}, 26-\mathrm{VII}-1933$, Roy 2880 (DAO); Gaspé-Sud Co., Grande-Rivière, 48 $21^{\circ}$ N, 64.41'W, 14-VII-1979, Cayouette J79-157 (DAO); Huntingdon Co., Huntingdon, $45^{\circ} 5^{\prime} \mathrm{N}, 74^{\circ} 10^{\prime} \mathrm{W}, 17$ VIII-1952, Bassett \& Hamel 2550 (DAO); Mégantic, Thetford mines, $46^{\circ} 5^{\prime} \mathrm{N}, 71^{\circ} 18^{\prime} \mathrm{W}, 16-\mathrm{VIIl}-1965$, Blais \& al. 10743 (DAO); Wolfe, Dudswell, Bishopton, $45^{\circ} 35^{\prime} \mathrm{N}$, 71'35'W, 12-VIII-1968, Hamel \& Brisson 15182 (DAO); Québec, $46^{\circ} 49^{\circ} \mathrm{N}, 71^{\circ} 14^{\prime} \mathrm{W}, 17-\mathrm{VII}-196$, Caron s.n. (DAO); Bonaventure, Escuminac, 48 $7^{\prime} \mathrm{N}, 66^{\circ} 29^{\prime} \mathrm{W}, 11$ VII-1972, Cinq-Mars 72-207 (DAO); Montmorency, $46^{\circ} 52^{\prime} \mathrm{N}, 71^{\circ} 9^{\prime} \mathrm{W}, 17-\mathrm{VII}-1980$, Jomphe 210 (DAO); Gaspé-Ouest Co., Grande-Rivière, $48^{\circ} 23^{\prime} \mathrm{N}, 64^{\circ} 30^{\prime} \mathrm{W}$, 14-VII-1979, Cayouette J79.157 (DAO); Rimouski, Sainte-Luce-sur-mer, $48^{\circ} 33^{\prime} \mathrm{N}, 68^{\circ} 23^{\prime} \mathrm{W}, 29-\mathrm{VII}-1977$, Charest 901 (DAO); Nicolet, $46^{\circ} 13^{\prime} \mathrm{N}, 72^{\circ} 37^{\prime} \mathrm{W},-1927$, Saint John s.n. (DAO).

USA. CoNNECTICUT: New Haven Co., Naugatuck, $41^{\circ} 29^{\prime} \mathrm{N}, 73^{\circ} 3^{\prime} \mathrm{W}, 16-\mathrm{VII}-1905$, Bristol 4837 (GH). MaINE: Penobscot Co., 4515'N, 6840'W, 3-VII-1900, Churchill s.n. (MO); Aroostook Co., Silver Ridge, 454' N, 6819'W, 11-VII-1939, Perkins s.n. (VT). MassachusetTs: Essex Co., Ipswich River Wildlife Sanctuary, 42 ${ }^{\circ} 39^{\prime} \mathrm{N}, 7^{\circ} 53^{\prime} \mathrm{W}, 13-\mathrm{VI}-1955$, Harris \& Bean 9954 (GH). MICHIGAN: Ann Arbor, $42^{\circ} 16^{\prime} \mathrm{N}$, 83 $43^{\prime} \mathrm{W}, 21-\mathrm{VII}-1954$, Carothers s.n. (MICH). VERMONT: Bennington, $42^{\circ} 52^{\prime} \mathrm{N}, 73^{\circ} 11^{\prime} \mathrm{W}, 1-\mathrm{VIII}-1937$, Ridlon s.n. (VT); Chittenden Co., Charlotte, 44'18'N, 73¹5' W, 24-VI-1969, Carse s.n. (VT); Washington Co., Barre, $44^{\circ} 11^{\prime} \mathrm{N}, 7^{\circ} 30^{\prime} \mathrm{W}, 8$-VII-1976, Ladd 1764 (VT).

6. Geranium sanguineum L., Sp. Pl.: 683 (1753)

Ind. loc.: "Habitat in Europae pratis siccis umbrosis"

Typus: "Habitat in Europae pratis siccis umbrosis" (lectotype, here designated,
Herb. Clifford 343 Geranium 1 Sheet A, BM colour image!) [choice made by B. Jonsell, and C. Jarvis]

Herbs perennial, $15-60 \mathrm{~cm}$ tall. Rootstock 9$15 \mathrm{~mm}$ diam., \pm horizontal, not tuberculate, not turnip-shaped. Stem erect, usually few per caudex branch, with patent eglandular hairs 0.8 $3.5 \mathrm{~mm}$ long (and glandular, hairs $0.1 \mathrm{~mm}$ long on the upper part). Basal leaves in a deciduous rosette; lamina 2.2-6.1 $\times 2.6-7 \mathrm{~cm}$, polygonal in outline, cordate, palmatifid (divided for 0.82 0.92 of its length), pilose, with appressed, eglandular hairs; segments 5-7, rhombic, 3-5 mm at the base, 3(-6)-lobed in distal half (ratio main-sinus length of the middle segment / middle-segment length $=0.35-0.5$ ); cauline leaves opposite; petioles to $5 \mathrm{~cm}$ long, with patent, eglandular hairs 0.8-2.5 mm long (and glandular hairs $0.1 \mathrm{~mm}$ long on the upper part); stipules 4-6 $\times 3-$ $5 \mathrm{~mm}$, lanceolate, with eglandular hairs on abaxial surface and on the margin, glabrous adaxially. Inflorescence a monochasial cyme; cymules 1-flowered, solitary; peduncles $3.2-11 \mathrm{~cm}$ long, with patent, eglandular hairs $0.8-2.5 \mathrm{~mm}$ long; bracteoles 2-3.5 × 1-2 mm, lanceolate, with eglandular hairs on abaxial surface and on the margin, glabrous adaxially; pedicels $1.5-5.5 \mathrm{~cm}$ long, with patent, eglandular hairs $0.8-2.5 \mathrm{~mm}$ long; pedicel and peduncle together overtopping the subtending leaf. Sepals 7-10 $\times 3-4.6 \mathrm{~mm}$ (ratio pedicel length $/$ sepal length $=2.6-3.9$ ), smooth, not accrescent, 3-5-nerved, with mucro 2-3.1 mm long, with scarious margins $0.5-1 \mathrm{~mm}$ wide, with patent, eglandular hairs $1-2 \mathrm{~mm}$ long, and appressed, eglandular $0.1 \mathrm{~mm}$ long on the abaxial side, glabrous adaxially. Petals 15-18 $\times$ $10-11 \mathrm{~mm}$ (ratio petal length / petal width $=1.4$ 1.6), erect-patent, emarginate (notch $1.5-2 \mathrm{~mm}$ deep), without claw, hairy on $1 / 5$ of their adaxial surface, ciliate on the basal margin, purplish. Stamens 10 , both whorls bearing anthers; filaments 6-8 mm long, not exserted, lanceolate, glabrous on both sides, ciliate on the proximal third, with hairs $0.2-0.5 \mathrm{~mm}$ long; anthers 2.4$2.6 \times 1-1.1 \mathrm{~mm}$. Nectaries glabrous. Gynoecium 8-9 mm long. Fruit $35-39 \mathrm{~mm}$ long, erect when immature; mericarps 4-5.1 $\times 2.1-3.5 \mathrm{~mm}$, without a strand of fibres, with 2-3 transverse 
ribs at apex, without longitudinal rib, without basal beak, with a basal callus, with \pm patent, eglandular hairs $0.2-1 \mathrm{~mm}$ long (mainly at the apex), brownish; rostrum 30-32 mm long, with a narrowed apex $4 \mathrm{~mm}$ long, with \pm patent, eglandular hairs 0.5-1 mm long; stigmatic remains ca. $3 \mathrm{~mm}$ long (ratio fruit length / stigmatic remains length $=10.6-13$ ), with 5 hairy lobes. Seeds 3.4$3.6 \times 2.4-2.5 \mathrm{~mm}$, finely reticulate, reddish; hilum $1 / 4$ as long as the perimeter. Cotyledons entire. $2 n=84, n=42$.

Flowering June-August. Waste grounds and roadsides; $0-400 \mathrm{~m}$. Europe, Caucasus, Western Asia \& Siberia; introduced from the Old World in Northeastern U.S.A. Fig. 13.

Illustrations. Fig. 14d. CAVANILLES (1787, tab. 76 fig. 1, fig. 3) [sub G. prostratum]; ROSS-CRAIG (1952, pl. 28); TOKARSKI (1972: 71, pl. 35).

This introduced species is unmistakable due to its 1-flowered cymules, long petals, and dissected leaves.
According to S. Welsh (in litt.) at BRY there is only one sheet of $G$. sanguineum from Utah and it is a cultivated specimen. No other evidence for the presence of $G$. sanguineum in Utah has been found (but see KARTESZ, 1998). The specimens supporting the records of $G$. sanguineum from Maine, New Hampshire, and Ohio (KARTESZ 1998) have not been studied.

\section{Representative specimens examined}

USA. ILLINoIs: Chicago, $41^{\circ} 51^{\prime} \mathrm{N}, 87^{\circ} 39^{\prime} \mathrm{W}, 1882$, Ohlendorf s.n. (F). MASSACHusETTS: Essex Co., An-

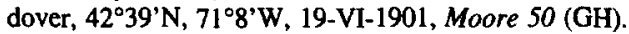
MICHIGAN: Ingham Co., Lansing, $42^{\circ} 43^{\prime} \mathrm{N}, 84^{\circ} 33^{\prime} \mathrm{W}$ (MSC); Benzie Co., River Road, $44^{\circ} 39^{\prime} \mathrm{N}, 86^{\circ} 1^{\prime} \mathrm{W}, 23-$ VIII-1982, Overlease 2802 (MICH); Schoolcraft Co., county road $437,42^{\circ} 6^{\prime} \mathrm{N}, 85^{\circ} 38^{\prime} \mathrm{W}, 28-\mathrm{VII}-1986$, Henson 2172 (MICH); Oakland Co., Bloomfield Village, $42^{\circ} 32^{\prime} \mathrm{N}, 83^{\circ} 13^{\prime} \mathrm{W}, 9-\mathrm{VI}-1931$, Farwell 8884 (MICH); Washtenaw Co., Dexter, North Lake, $42^{\circ} 20^{\prime} \mathrm{N}, 8^{\circ} 53^{\prime} \mathrm{W}$, 26-VII-1954, Whitmire s.n. (MICH). NEW JERSEY: Ocean Co., Manahawkin, $39^{\circ} 42^{\prime} \mathrm{N}, 74^{\circ} 16^{\prime} \mathrm{W}, 21-\mathrm{VII}-1923$, Long 27981 (GH, PH). New YoRK: Franklin Co., Loon

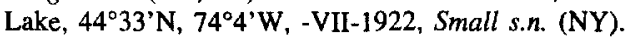
Pennsylvania: Delaware Co., Swarthmore, $39^{\circ} 54^{\prime} \mathrm{N}$, $75^{\circ} 21^{\prime}$ W, -VI-, Natkins s.n. (PH): Bucks Co.,

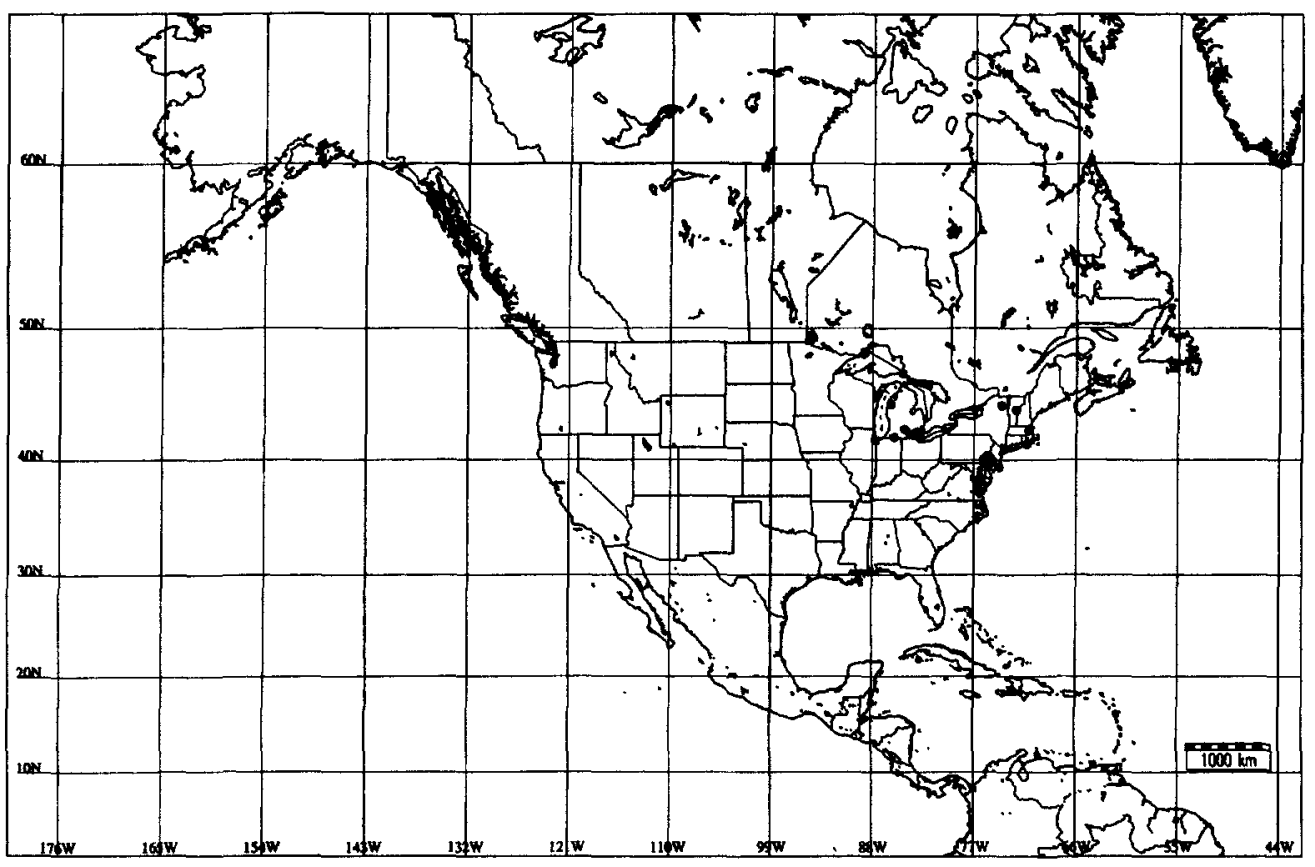

Fig. 13.-Distribution of Geranium sanguineum in North America. 

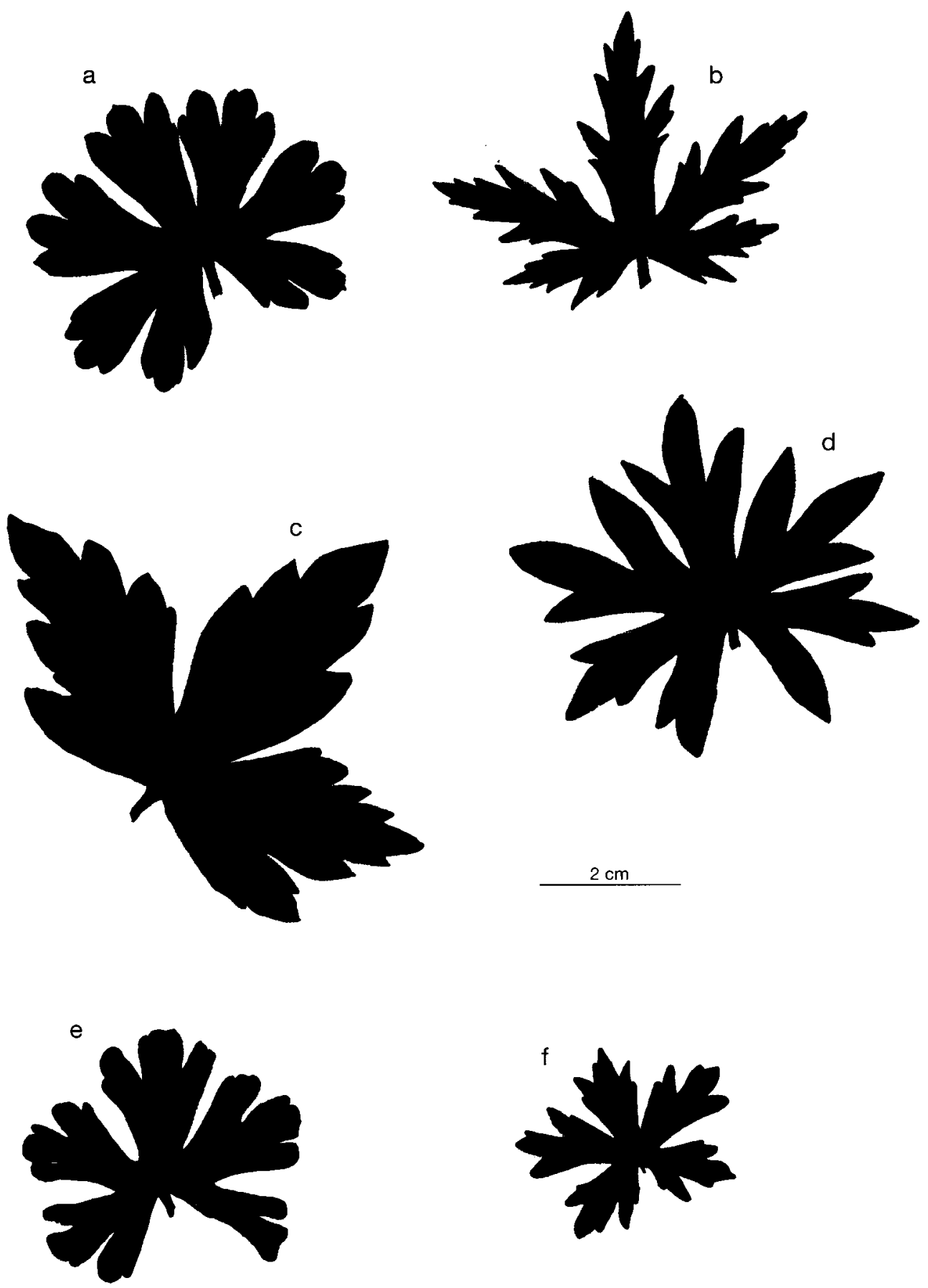

Fig. 14,-Silhouettes of laminas of: a, Geranium potentilloides [Aedo 4473 (MA)]; b, G. sibiricum [Castroviejo 14137 (MA)]; c, G. thunbergii [Aedo 3046 (MA)]; d, G. sanguineum [Ferrero \& al. s.n. (MA)]; e, G. retrorsum [Burtt Davy 1650 (UC)]; f, G. solanderi [Tracy 3238 (UC)]. 
Churchville, $40^{\circ} 11^{\prime} \mathrm{N}, 75^{\circ} 1^{\prime} \mathrm{W}, 29-\mathrm{VI}-1961$, Long s.n. (PH); Berks Co., Wyomissing, 40 $19^{\prime} \mathrm{N}, 75^{\circ} 57^{\prime} \mathrm{W}, 24-$ VI-1950, Wilkens 8146 (PH); Lehigh Co., Crackersport. 4036' N, 75'33'W, 7-VII-1956, Schaefer 50699 (PH). Rhode Island: Newport Co., Middletown, 41 $32^{\prime} \mathrm{N}$, $71^{\circ} 17^{\prime} \mathrm{W}$, 5-VII-1909, Williams s.n. (GH). VERMONT: Washington Co., Barre City, $44^{\circ} 12^{\prime} \mathrm{N}, 72^{\circ} 29^{\prime} \mathrm{W}, 14-\mathrm{VI}$ 1976, Ladd 1364 (VT).

\section{Geranium potentilloides L'Hér. ex DC.,}

Prodr. 1: 639 (1824)

Ind. loc.: "in Novâ-Hollandiâ. Banks. (v.s.)"

Typus: Novâ-Hollandiâ. Banks s.n. [lectotype, designated by CAROLIN (1965: 337),

$\mathrm{G}$; isolectotype, $\mathrm{BM}$ !]

Herbs perennial, $15-60 \mathrm{~cm}$ tall. Rootstock 3-5 mm diam., \pm vertical, not tuberculate, not turnip-shaped. Stem decumbent or ascending, usually few per caudex branch, with patent to retrorse, not appressed, eglandular hairs 0.3$1.2 \mathrm{~mm}$ long. Basal leaves in a deciduous rosette; lamina 2.8-4.3 $\times 3.5-5.2 \mathrm{~cm}$, polygonal in outline, cordate, palmatifid (divided for 0.71-0.75 of its length), pilose, with appressed, eglandular hairs; segments 5-7, obtri- angular, $4-5 \mathrm{~mm}$ at the base, 3-5-lobed at the apex (ratio main-sinus length of the middle segment $/$ middle-segment length $=0.15$ 0.18 ); cauline leaves opposite; petioles to 11 $\mathrm{cm}$ long, with patent to retrorse, eglandular hairs 0.2-1.1 mm long; stipules 5-6 $\times$ 0.5-1 $\mathrm{mm}$, lanceolate, with eglandular hairs on both surfaces, and on the margin, ending in 1-3 bristles $0.4-0.6 \mathrm{~mm}$ long. Inflorescence a monochasial cyme; cymules 1-flowered, solitary; peduncles $1.5-3.6 \mathrm{~cm}$ long, with patent to retrorse, not appressed, eglandular hairs 0.3$1.2 \mathrm{~mm}$ long; bracteoles $3.4 \times 0.4-0.6 \mathrm{~mm}$, linear-lanceolate, with eglandular hairs on abaxial surface and on the margin, glabrous adaxially; pedicels $1.2-2.2 \mathrm{~cm}$ long, with patent to retrorse, not appressed, eglandular hairs 0.3-1.1 mm long; pedicel and peduncle together overtopping the subtending leaf. Sepals 4.7-5.3 $\times 2-2.1 \mathrm{~mm}$ (ratio pedicel length $/$ sepal length $=2.5-4.1$ ), smooth, not accrescent, 3-nerved, with mucro $0.4-0.9 \mathrm{~mm}$ long, with scarious margins $0.1 \mathrm{~mm}$ wide, with antrorse, \pm appressed, eglandular hairs

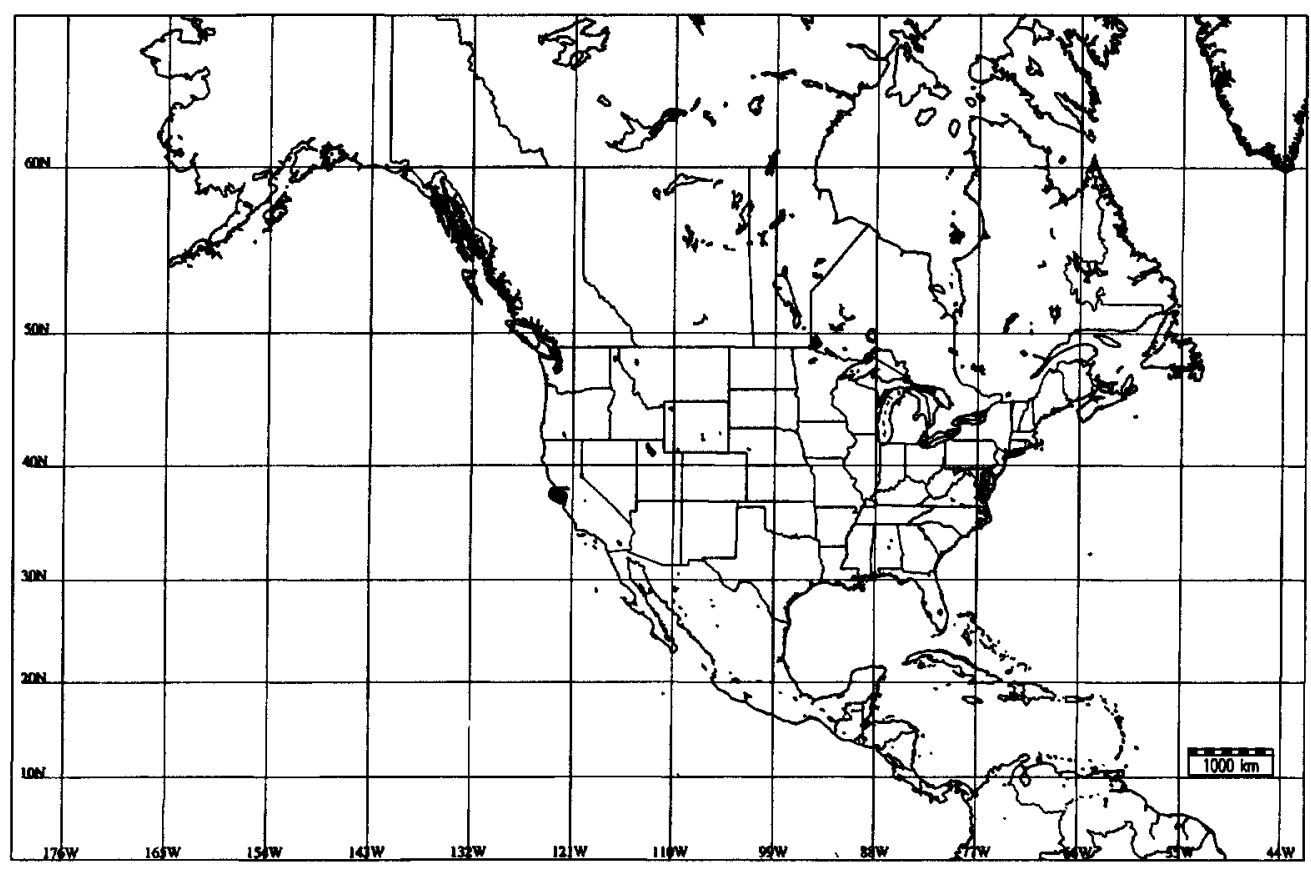

Fig. 15.-Distribution of Geranium potentilloides in North America. 
0.1-0.2 mm long on the abaxial side (and eglandular hairs $0.5-1 \mathrm{~mm}$ long on the margin), glabrous adaxially. Petals 6-9 $\times 3-5.5 \mathrm{~mm}$ (ratio petal length / petal width $=1.6-1.8$ ), erectpatent, entire or slightly notched, without claw, glabrous on both sides, ciliate on the basal margin, white to pinkish. Stamens 10 , both whorls bearing anthers; filaments $3.5 \mathrm{~mm}$ long, not exserted, lanceolate with an abruptly narrowed apex, glabrous on both sides, ciliate on the proximal half, with hairs $0.1-0.2 \mathrm{~mm}$ long; anthers 0.6-0.7 $\times 0.5 \mathrm{~mm}$. Nectaries glabrous. Gynoecium $4 \mathrm{~mm}$ long. Fruit 15-16 mm long, erect; mericarps $2-3 \times 1-1.5 \mathrm{~mm}$, without a strand of fibres, smooth, without longitudinal rib, without basal beak, with a basal callus, with \pm patent, eglandular hairs 0.1-0.5 mm long, and glandular hairs $0.1 \mathrm{~mm}$ long, brownish; rostrum 10-11 mm long, with a narrowed apex $0.5 \mathrm{~mm}$ long, with erectpatent, eglandular hairs 0.1-0.2 mm long; stigmatic remains $1.5-1.6 \mathrm{~mm}$ long (ratio fruit length / stigmatic remains length $=9.3-10.6$ ), with 5 glabrous lobes. Seeds 1.8-2 $\times 1-1.2$ $\mathrm{mm}$, finely reticulate, brownish; hilum 1/4 as long as the perimeter. Cotyledons entire.

Flowering May-August. Weedy areas, and edge of forests; $0-100 \mathrm{~m}$. Australia \& New Zealand; introduced in Southwestern U.S.A. Fig. 15.

Illustrations. Fig. 14a; HARDEN (1992: 24).

Geranium potentilloides is characterised by its small petals and $\mathbf{1 - f l o w e r e d ~ c y m u l e s . ~ I t ~}$ might at first be mistaken for $G$. sibiricum, but the former has smaller fruits and seeds, and leaves with middle segment obtriangular, 3(5)-lobed at the apex, while the latter has large fruits and seeds, and leaves with middle segment rhombic, 7-9-lobed in the distal half.

\section{Representative specimens examined}

USA. California: Alameda, $37^{\circ} 45^{\prime} \mathrm{N}, 122^{\circ} 14^{\prime} \mathrm{W}$, 11-V-1891, Greene s.n. (F); Marin Co., Bear Valley, 37 $59^{\prime} \mathrm{N}, 122^{\circ} 48^{\prime} \mathrm{W}, 29-\mathrm{V}-1898$, Eastwood s.n. (F); Marin Co., Bolema Club, between Bolinas and Olema, $37^{\circ} 59^{\prime} \mathrm{N}, 122^{\circ} 45^{\prime} \mathrm{W}, 3-\mathrm{V}-1965, \mathrm{McHoul}$ s.n. (UC); Marin Co., near Olema, $38^{\circ} 2^{\prime} \mathrm{N}, 122^{\circ} 47^{\prime} \mathrm{W}$, -VIII-, Burtt Davy 4342 (UC); Marin Co., Point Reyes National Seashore, $38^{\circ} 4^{\prime} \mathrm{N}, 122^{\circ} 53^{\prime} \mathrm{W}, 19-\mathrm{XI}-1967$, Porter \& Porter 1579 (MO).
8. Geranium sibiricum L., Sp. Pl.: 683 (1753)

Ind. loc.: "Habitat in Sibiria".

Typus: "Habitat in Sibiria" [lectotype, designated by YEO (1992b: 189), LINN-85887 colour slide!]

Herbs perennial, $15-90 \mathrm{~cm}$ tall. Rootstock 3-5 mm diam., \pm vertical, not tuberculate, not turnip-shaped. Stem ascending, usually few per caudex branch, with retrorse, appressed, eglandular hairs 0.3-0.6 mm long. Basal leaves in a deciduous rosette; lamina 2.9-5.8 $\times 3.4-7.2 \mathrm{~cm}$, polygonal in outline, cordate, palmatifid (divided for 0.8-0.89 of its length), pilose, with appressed, eglandular hairs; segments 5-7, rhombic, 3-5 mm at the base, 7-14lobed in distal half (ratio main-sinus length of the middle segment / middle-segment length $=0.09-0.22$ ); cauline leaves opposite; petioles to $12 \mathrm{~cm}$ long, with patent to retrorse, eglandular hairs 0.5-1.1 mm long; stipules 5-8 $\times 1$ $1.5 \mathrm{~mm}$, lanceolate, with eglandular hairs on abaxial surface and on the margin, glabrous adaxially. Inflorescence a monochasial cyme; cymules 1 -flowered, solitary; peduncles $(0-$ )2-4.5 cm long, with retrorse, \pm appressed, eglandular hairs $0.2-0.5 \mathrm{~mm}$ long; bracteoles 2.5-3.5 $\times 0.5-1 \mathrm{~mm}$, linear-lanceolate, with eglandular hairs on abaxial surface and on the margin, glabrous adaxially; pedicels 2.3-3.5 $\mathrm{cm}$ long, with retrorse, \pm appressed, eglandular hairs 0.2-0.5 mm long; pedicel and peduncle together overtopping the subtending leaf. Sepals 5-6.5 × 2-3.5 mm (ratio pedicel length / sepal length $=3.8-7$ ), smooth, not accrescent, 3-nerved, with mucro 0.9-1.5 mm long, with scarious margins $0.2-0.3 \mathrm{~mm}$ wide, with antrorse, eglandular \pm appressed hairs $0.2-1$ $\mathrm{mm}$ long on the abaxial side, glabrous adaxially. Petals 5-7 $\times 1.5-2 \mathrm{~mm}$ (ratio petal length / petal width $=2.5$ ), erect-patent, entire or slightly notched, without claw, glabrous on both sides, ciliate on the basal margin, white or pale pink with purplish veins. Stamens 10 , both whorls bearing anthers; filaments 1.5-2 $\mathrm{mm}$ long, not exserted, lanceolate with an abruptly narrowed apex, glabrous on both sides, ciliate on the proximal half, with hairs 
$0.1 \mathrm{~mm}$ long; anthers $0.4 \times 0.4 \mathrm{~mm}$. Nectaries glabrous. Gynoecium $3 \mathrm{~mm}$ long. Fruit 16-19 $\mathrm{mm}$ long, erect when immature; mericarps $3.5-4 \times 1.5-1.8 \mathrm{~mm}$, without a strand of fibres, smooth, without longitudinal rib, without basal beak, with a basal callus, with \pm patent, eglandular hairs 0.1-1 mm long, and glandular hairs $0.1 \mathrm{~mm}$ long, brownish; rostrum 12 $15 \mathrm{~mm}$ long, with a narrowed apex $0.5 \mathrm{~mm}$ long, with antrorse, eglandular hairs ca. 0.1$0.2 \mathrm{~mm}$ long; stigmatic remains c. $1 \mathrm{~mm}$ long (ratio fruit length $/$ stigmatic remains length $=$ 16-18), with 5 hairy lobes. Seeds 2.6-2.7 $\times$ 1.3-1.4 mm, finely reticulate, brownish; hilum 1/4 as long as the perimeter. Cotyledons entire. $2 n=28$.

Flowering May-August. Waste places and roadsides; 0-400 m. Europe, Caucasus, Western Asia, Siberia, \& China; introduced from Old World in North-Central U.S.A. \& Northeastern U.S.A. Fig. 16.

Illustrations. Fig. 14b. JACQUIN (1770-71, tab. 19).
According to PORTER (1972: 449) records of $G$. sibiricum for California should be considered as $G$. potentilloides. The specimens supporting records of $G$. sibiricum from Connecticut, and New Jersey (KARTESZ 1998) have not been studied.

\section{Representative specimens examined}

USA. ILLINOIS: Urbana, $40^{\circ} 6^{\prime} \mathrm{N}, 88^{\circ} 12^{\prime} \mathrm{W}$, Gleason 34 (GH); Winnebago Co., near Seward Bluffs, $42^{\circ} 14 ' \mathrm{~N}$, $89^{\circ} 21^{\prime}$ W, 12-IX-1947, Fell \& Fell f47-284 (F). MaSSACHUSETTS: Hampshire Co., Mount Holyoke College campus, South Hadley, $42^{\circ} 18^{\prime} \mathrm{N}, 72^{\circ} 35^{\prime} \mathrm{W}, 4$-VIII-1979. Ahles 87219 (MA VT). Minnesota: Goodhue Co.,

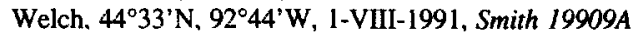

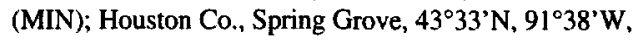
-X-. Ownbey s.n. (MIN). NEW YoRK: Dutchess Co., Wappingers Falls, $41^{\circ} 35^{\prime} \mathrm{N}, 73^{\circ} 54^{\prime} \mathrm{W}, 30$-VII-1977, Churchill 77715 (MSC); New York City, Browdway, $40^{\circ} 45^{\prime} \mathrm{N}, 73^{\circ} 48^{\circ} \mathrm{W}, 2-\mathrm{X}-1912$, Al? Jrill 8633 (VT); Rockland Co., Ramapo, $41^{\circ} 8^{\prime} \mathrm{N}, 74^{\circ} 10^{\prime} \mathrm{W}, 18$-VIII1954. Lehr 459 (NY). Pennsylvania: Centre Co., at east edge of State College, $40^{\circ} 47^{\prime} \mathrm{N}, 77^{\circ} 51^{\prime} \mathrm{W}, 11-\mathrm{IX}-1942$, Wahl 1333 (MO); Dauphin Co., $4 \mathrm{mi}$ W Dauphin, $40^{\circ} 22^{\prime} \mathrm{N}, 76^{\circ} 55^{\prime} \mathrm{W}, 20$-VIII-1952, Berkheimer 14737 (PH); Delaware Co., Krrklyn, 39 $58^{\prime}$ N. $75^{\circ} 17^{\prime} \mathrm{W}, 30-\mathrm{IX}-$ 1912, Keeney s.n. (PH); Lancaster Co., Millersville, $39^{\circ} 59^{\circ} \mathrm{N}, 76^{\circ} 21^{\prime} \mathrm{W}, 23-\mathrm{VIII}-1940$, Tanger 4000 (PH):

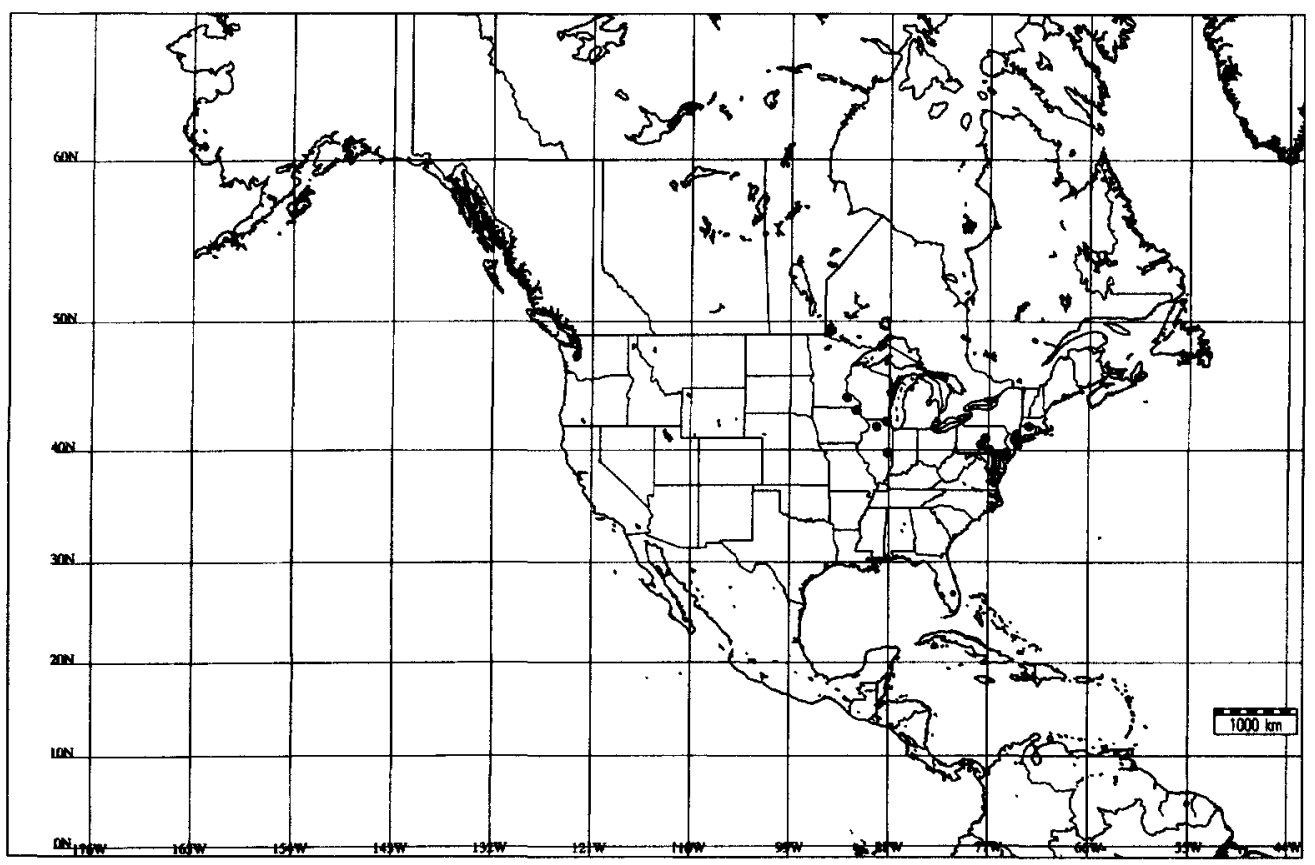

Fig. 16.-Distribution of Geranium sibiricum in North America. 
Lycoming Co., Waterville, $41^{\circ} 18^{\prime} \mathrm{N}, 77^{\circ} 21^{\prime} \mathrm{W}, 1$-VIII1956, Wahl 7657 (PH); Montgomery Co., Elkins Park, $40^{\circ} 4^{\prime} \mathrm{N}, 75^{\circ} 7^{\prime} \mathrm{W}, 6$-VIII-1923, Dreisbach 1715 (MICH); Montgomery Co., Haverford, $40^{\circ} 0^{\prime} \mathrm{N}, 75^{\circ} 17^{\prime} \mathrm{W}, 20-\mathrm{IX}-$ 1939, Schaeffer s.n. (PH); Philadelphia Co., Germantown, $40^{\circ} 2{ }^{\prime} \mathrm{N}, 75^{\circ} 10^{\prime} \mathrm{W}, 24-\mathrm{V}$ - 1942 , Long 58302 (MIN).

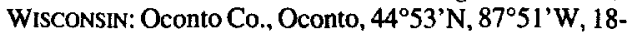
IX-1993, Harriman 19200 (F); Racine Co., W of Burlington, $42^{\circ} 40^{\circ} \mathrm{N}, 88^{\circ} 16^{\circ} \mathrm{W}, 10-\mathrm{VIII}-1968$, Rowlatt s.n. (MOR).

9. Geranium thunbergii Siebold ex Lindl. \& Paxton in Paxt. Fl. Gard. 1(12): 186 fig. 115 (1851)

Ind. loc.: "[Cult. Garden of the Horticultural Society of London] ('H.H.S.')"

Typus: Cult. Garden of the Horticultural Society of London (lectotype. designated by Yeo 1992b: 185, CGE-herb. Lindley)

Herbs perennial, $40-70 \mathrm{~cm}$ tall. Rootstock 6-10 mm diam., vertical, not tuberculate, not turnip-shaped. Stem decumbent or ascending, usually solitary, with patent or retrorse, appressed eglandular hairs $0.3-1.1 \mathrm{~mm}$ long. Basal leaves in a deciduous rosette; lamina 3.5-6.5 × 4-8.4 cm, polygonal in outline, cordate, palmatifid (divided for $0.72-0.8$ of its length), pilose, with appressed, eglandular hairs; segments $5(-7)$, rhombic, $7-12 \mathrm{~mm}$ at the base, 5-7-lobed in distal half (ratio mainsinus length of the middle segment / middlesegment length $=0.14-0.2$ ); cauline leaves opposite: petioles to $12 \mathrm{~cm}$ long. with patent to retrorse, eglandular hairs $0.3-1.1 \mathrm{~mm}$ long; stipules 5-7 $\times 2-2.5 \mathrm{~mm}$, lanceolate to subulate, with eglandular hairs on abaxial surface and on the margin, glabrous adaxially. Inflorescence a monochasial cyme; cymules 2flowered, solitary; peduncles $2.3-5.8 \mathrm{~cm}$ long, with patent, eglandular hairs $0.2-0.8 \mathrm{~mm}$ long, sometimes with glandular hairs 0.4-0.8 $\mathrm{mm}$ long; bracteoles $2-3 \times 0.5 \mathrm{~mm}$, linearlanceolate, with eglandular hairs on both sides and on the margin; pedicels $1.4-2.4 \mathrm{~cm}$ long, with patent, eglandular hairs $0.2-0.8 \mathrm{~mm}$ long, sometimes with glandular hairs 0.4-0.8 $\mathrm{mm}$ long; pedicel and peduncle together overtopping the subtending leaf. Sepals 6-7 $\times 2.5$ $3.5 \mathrm{~mm}$ (ratio pedicel length $/$ sepal length $=$ 2.3-4), smooth, not accrescent, 3-5-nerved, with mucro $0.5-1 \mathrm{~mm}$ long, with scarious margins $0.2-0.3 \mathrm{~mm}$ wide, with antrorse, appressed, eglandular hairs $0.1-0.3 \mathrm{~mm}$ long and patent, glandular hairs $0.6-1.1 \mathrm{~mm}$ long on the abaxial side, glabrous adaxially. Petals $7-10 \times$ $5-5.5 \mathrm{~mm}$ (ratio petal length $/$ petal width $=$ 1.4-1.8), erect-patent, retuse, without claw, hairy on the base of adaxial side, glabrous on the abaxial side, ciliate on the basal margin, white or purplish. Stamens 10, both whorls bearing anthers; filaments $4-6 \mathrm{~mm}$ long, not exserted. lanceolate with an abruptly narrowed apex, pilose on the abaxial side, ciliate on the proximal half, with hairs $0.1-0.3 \mathrm{~mm}$ long; anthers $0.8-0.9 \times 0.5 \mathrm{~mm}$. Nectaries glabrous. Gynoecium 3-3.5 mm long. Fruit 21-23 mm long, erect when immature; mericarps 3-3.5 $\times 2 \mathrm{~mm}$, without a strand of fibres, smooth, without longitudinal rib, without basal beak, with a basal callus, with \pm patent, eglandular hairs 0.2-0.6 mm long, and glandular 1.1-1.7 mm long, blackish; rostrum 16$17 \mathrm{~mm}$ long, without a narrowed apex. with patent, eglandular hairs ca. $0.1 \mathrm{~mm}$ long and patent, glandular hairs 0.4-0.6 mm long; stigmatic remains $2-3 \mathrm{~mm}$ long (ratio fruit length / stigmatic remains length $=7.3-11$ ), with 5 hairy lobes. Seeds $1.9-2 \times 1.2 \mathrm{~mm}$, finely reticulate, brownish; hilum 1/6 as long as the perimeter. Cotyledons unknown.

Flowering August-October. Open woods, waste grounds and weedy areas; $0-100 \mathrm{~m}$. North China, Taiwan, and Japan; introduced in Northeastern U.S.A. Fig. 17.

Illustrations. Fig. 14c. Yeo (1992a: 117 fig. 9.52).

Geranium thunbergii is a weedy species that varies considerably in density and distribution of glandular hairs. Glandular hairs are usually abundant on sepal abaxial surface. However, some specimens display scarce glandular hairs only at sepal base. Moreover, peduncles and pedicels have sometimes glandular hairs. It might be mistaken for $G$. nepalense Sweet, a related species which differs from $G$. thunbergii by the lack of any glandular hairs, and in being slender, smallerleaved and slightly smaller-flowered (YEO 1992a: 118). 


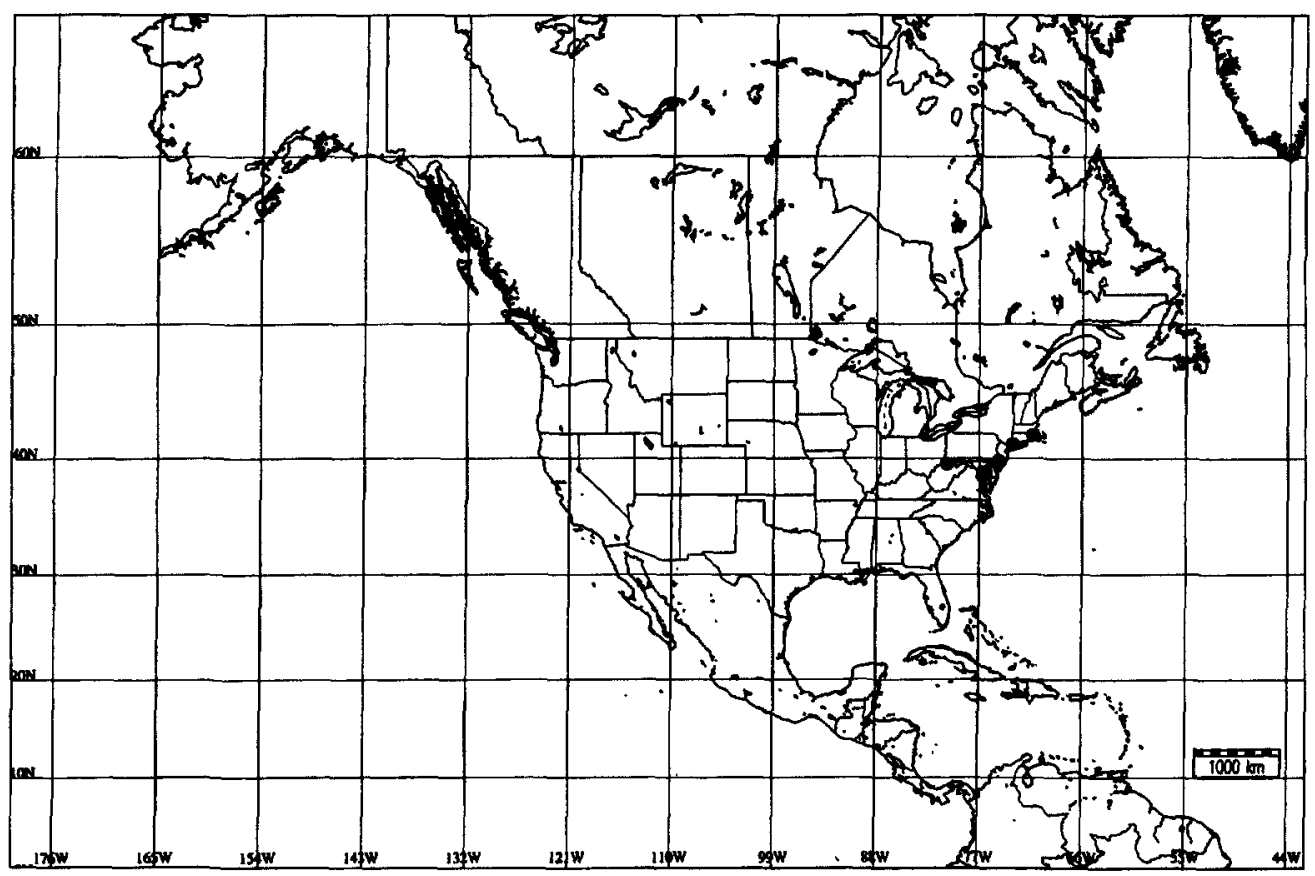

Fig. 17.-Distribution of Geranium thunbergii in North America.

Geranium versicolor L. [G. striatum L., nom. illeg.] was recorded from Pennsylvania by RHOADS \& KLEIN (1993: 259). No specimen from this state has been located in the studied herbaria. However, a sheet from Pennsylvania, labelled as $G$. striatum, has been identified as $G$. thunbergii, which is not in RHOADS \& KLEIN's (1993) catalogue of Pennsylvania. This would suggest a confusion between both species in this catalogue. All of the Pennsylvania occurrences probably were the result of someone disposing of garden trimmings along a stream or roadside.

\section{Representative specimens examined}

USA. CONNECTICUT: Litchfield Co., New Milford, $41^{\circ} 34^{\prime}$ N, $73^{\circ} 24^{\prime} \mathrm{W}, 11-\mathrm{VII}-1980$, Porges s.n. (GH). MARYLAND: Baltimore, $39^{\circ} 17^{\prime} \mathrm{N}, 7^{\circ} 36^{\prime} \mathrm{W}, 13-\mathrm{IX}-1952$, Fessenden s.n. (PH); Baltimore Co., slopes at Gunpowder Falls State Park $\mathrm{nr}$ end of Schroeder Avenue, 4-X1981, Hill \& Cress 10818 (MO). MASSACHUSETTS: Norfolk Co., Wellesley, $42^{\circ} 17^{\prime} \mathrm{N}, 71^{\circ} 17^{\prime} \mathrm{W},-\mathrm{IX}-$, Hunnewell 18089 (GH). Pennsylvania: Alleghny Co., Pittsburgh, $40^{\circ} 26^{\prime} \mathrm{N}, 79^{\circ} 59^{\prime} \mathrm{W}, 8-\mathrm{V}-1950$, Buker s.n. (CM); Bucks Co., George School, $40^{\circ} 12^{\prime}$ N, 7456'W, 29-VI-1932, Benner s.n. (GH); Lawrence Co., New Castle, Cascade

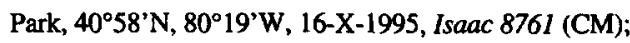
Washington Co., $3.7 \mathrm{~km}$ ESE of Majorsville, $39^{\circ} 57^{\prime} \mathrm{N}$, $80^{\circ} 28^{\prime} \mathrm{W}, 27-\mathrm{IX}-1984$, Thompson 2090 (PH).

10.Geranium retrorsum L'Hér. ex DC., Prodr. 1: 644 (1824)

G. dissectum var. retrorsum (L'Hér. ex DC.) Hook. f., Fl. Nov.-Zel. 1: 39 (1852) G. pilosum var. retrorsum (L'Hér. ex DC.) Jeps., Man. Fl. Pl. Calif.: 589 (1925)

Ind. loc.: "in Novâ-Zelandiâ. Banks. (v. s. cum semill. immat.)"

Typus: Novâ-Zelandiâ, Banks s.n. (lectotype, designated by CAROLIN 1965: 349, $\mathrm{G}$; isolectotype, $\mathrm{BM}$ !)

Herbs perennial, 30-60 cm tall. Rootstock 10-16 mm diam., vertical, not tuberculate, turnip-shaped. Stem decumbent or ascending, usually few per caudex branch, with retrorse, appressed, eglandular hairs 0.3-0.5 $\mathrm{mm}$ long. Basal leaves in a \pm deciduous rosette; lamina 1.6-4.2 $\times 2-4.4 \mathrm{~cm}$, polygonal to orbicular in outline, cordate, palmatifid (divided for $0.55-0.8$ of its length), pilose, 
with appressed, eglandular hairs; segments $5(-7)$, rhombic to obtriangular, $3-4 \mathrm{~mm}$ at the base, (3-)6-10-lobed in distal half (ratio main-sinus length of the middle segment / middle-segment length $=0.23-0.4$ ); cauline leaves opposite; petioles to $7 \mathrm{~cm}$ long, with retrorse, appressed, eglandular hairs $0.2-0.5$ $\mathrm{mm}$ long; stipules $4-7 \times 0.8-3 \mathrm{~mm}$, lanceolate, with eglandular hairs on abaxial surface and on the margin, glabrous adaxially. Inflorescence a monochasial cyme; cymules 2flowered, solitary; peduncles $0.7-2.7 \mathrm{~cm}$ long, with retrorse, appressed, eglandular hairs 0.2-0.5 mm long; bracteoles 2.5-6 $x$ 0.4-0.8 mm, linear-lanceolate, with eglandular hairs on abaxial surface and on the margin, glabrous adaxially; pedicels $0.7-2 \mathrm{~cm}$ long, with retrorse, appressed, eglandular hairs 0.2-0.5 mm long; pedicel and peduncle together overtopping the subtending leaf. Sepals 3.5-5 $\times 2-3.2 \mathrm{~mm}$ (ratio pedicel length / sepal length $=1.9-5$ ), smooth, accrescent, 3nerved, with mucro $0.7-1 \mathrm{~mm}$ long, with scarious margins $0.1-0.2 \mathrm{~mm}$ wide, with eg- landular hairs 0.1-0.4 mm long on the abaxial side (and eglandular hairs 0.5-1 mm long on the margin), glabrous adaxially. Petals $4.5-10 \times 2.3-4 \mathrm{~mm}$ (ratio petal length / petal width $=1.95-2.5$ ), erect-patent, entire or slightly notched, without claw, glabrous on both sides, ciliate on the basal margin, purplish. Stamens 10, both whorls bearing anthers; filaments $3 \mathrm{~mm}$ long, not exserted, lanceolate, glabrous on both sides, ciliate on the basal margin, with hairs up to $0.1-0.2 \mathrm{~mm}$ long; anthers $0.6 \times 0.5 \mathrm{~mm}$. Nectaries glabrous. Gynoecium 3-3.5 mm long. Fruit 14-19 mm long, erect; mericarps 2.7-3.2 $\times$ $1.2-1.5 \mathrm{~mm}$, without a strand of fibres, smooth, without longitudinal rib, without basal beak, with a basal callus, with erectpatent, eglandular hairs 0.1-0.4 mm long, brownish; rostrum 10-13 mm long, without a narrowed apex, with erect-patent, eglandular hairs 0.1-0.4 mm long; stigmatic remains 1$1.9 \mathrm{~mm}$ long (ratio fruit length / stigmatic remains length $=10-16$ ), with 5 hairy lobes. Seeds $2-2.5 \times 1.2-1.3 \mathrm{~mm}$, finely reticulate,

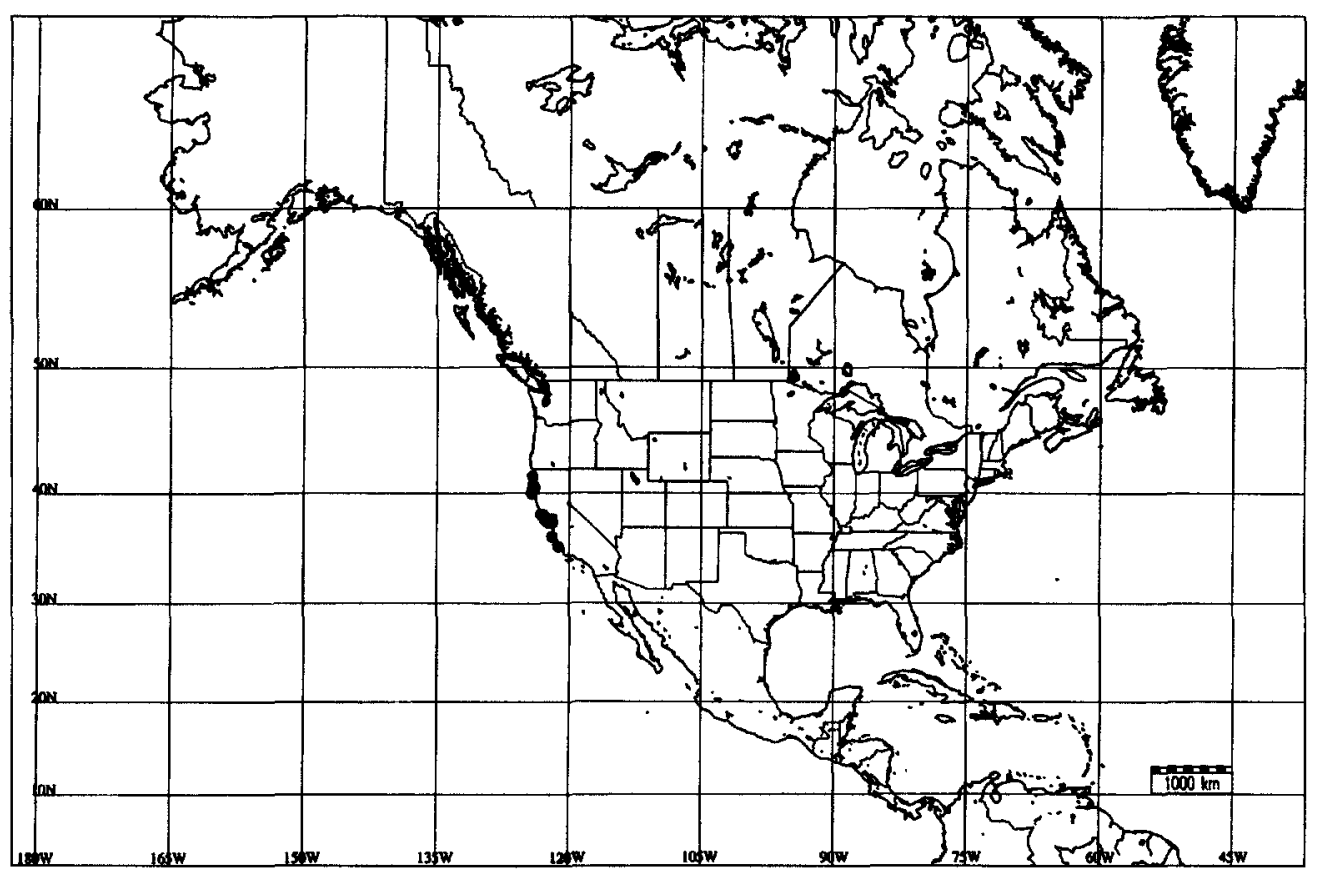

Fig. 18.-Distribution of Geranium retrorsum in North America. 
brownish; hilum 1/6 as long as the perimeter. Cotyledons entire.

Flowering March-October. Weedy areas and roadsides; $0-200 \mathrm{~m}$. Australia \& New Zealand; introduced in Southwestern U.S.A. Fig. 18.

Illustrations. Fig. 14e. HARDEN (1992: 25).

Geranium retrorsum and $G$. solanderi are closed species sharing a turnip-shaped rootstock. The former has pedicels with retrorse, appressed, eglandular hairs and a sepal mucro $0.7-1 \mathrm{~mm}$ long (without bristles), while the latter shows pedicels with patent or reflexed (never appressed) eglandular hairs and a short sepal mucro ending in 1-3 bristles. Additionally, $G$. retrorsum differs from $G$. solanderi by its large and more lobbed leaves, large mericarps and seeds.

HANKS \& SMALl (1907: 10) recorded $G$. glabratum (Hook. f.) Small [ $=$ G. homeanum Turcz.] from California. Major Californian floras did not incorporate G. glabratum in their Geranium accounts (JEPSON 1925; MUNZ 1959; TAYLOR 1993). ABRAMS (1951: 4) includes $G$. glabratum under $G$. retrorsum, but he did not clarify its status. Based on the fact that there is no specimen supporting this record, and on HANKS \& SMALL's (1907) description of "G. glabratum" the latter should be considered a mistake with $G$. retrorsum.

\section{Representative specimens examined}

USA. CalifornIA: Alameda Co., San Leandro Bay Shoreline, Oakland, $37^{\circ} 48^{\prime} \mathrm{N}, 122^{\circ} 16^{\prime} \mathrm{W}, 25-\mathrm{VII}-1991$, Ertter 10725 (UC); Contra Costa Co., Mount Diablo Regional Park, Pine Pond, 3751'N, 121 $1^{\circ} 55^{\prime} \mathrm{W}, 8$-IV-1987, Ertter 17410 (MA); Del Norte Co., Crescent City, $41^{\circ} 45^{\prime}$ N, $124^{\circ} 12^{\prime} \mathrm{W}, 2-\mathrm{VI}-1925$, Parks 8360 (UC); Humboldt Co., Blue Lake, $40^{\circ} 52^{\prime} \mathrm{N}, 123^{\circ} 58^{\prime} \mathrm{W}, 13-\mathrm{VI}-$ 1909, Tracy 2990 (UC); Humboldt Co., Eureka, $40^{\circ} 48^{\prime} \mathrm{N}, 124^{\circ} 9^{\prime} \mathrm{W}, 23-\mathrm{V}-1909$. Tracy 2977 (UC); Marin Co., Hwy 1/101 interchange along Richardson Bay, $37^{\circ} 52^{\prime} \mathrm{N}, 122^{\circ} 29^{\circ} \mathrm{W}, 8-\mathrm{IV}-1987$, Anderson 3344 (MA); Marin Co., road between Bolivar and Olema, $38^{\circ} 2^{\prime} \mathrm{N}$, $122^{\circ} 47^{\circ}$ W, 3-VII-1898, Eastwood s.n. (UC); Monterrey Co., Carmel Bay, $36^{\circ} 32^{\prime} \mathrm{N}, 121^{\circ} 57^{\prime} \mathrm{W},-$ IX-, Elmer 4046 (UC); San Francisco Co., Berkeley, Dwinelle Hall, $37^{\circ} 52^{\prime} \mathrm{N}, 122^{\circ} 15^{\prime} \mathrm{W}, 10-\mathrm{VII}-1981$, Dempster 4478 (JEPS); San Francisco Co., Presidio, $37^{\circ} 48^{\prime} \mathrm{N}$, $122^{\circ} 27^{\prime}$ W, -V-, Michener \& Bioletti 2004 (MO); San Luis Obispo Co., Piedras Blancas Point, $35^{\circ} 39^{\prime} \mathrm{N}$,
121 16'W, 18-IV-1947, Hoover 6960 (UC); Sonoma Co., Duncan's Mills, $38^{\circ} 27^{\prime} \mathrm{N}, 123^{\circ} 3^{\prime} \mathrm{W}, 13-\mathrm{III}-1896$, Burtt Davy 1650 (UC); Sonoma Co., Guerneville, $38^{\circ} 30^{\prime} \mathrm{N}, 122^{\circ} 59^{\prime} \mathrm{W}, 13-\mathrm{VIII}-1908$, Condit 130 (UC); Sonoma Co., Jenner, $38^{\circ} 30^{\circ} \mathrm{N}, 123^{\circ} 14^{\prime} \mathrm{W}, 31-\mathrm{V}-1937$, Reed $216(\mathrm{P})$.

11.Geranium solanderi Carolin in Proc. Linn. Soc. New South Wales ser. 2, 89: 350 (1965)

Geranium pilosum Sol. in G. Forst., Fl. Ins. Austr.: 91 (1786), nom. nud.

Geranium pilosum Sol. ex Willd., Sp. Pl. 3(1): 706 (1801) [syn. subst.], nom. illeg., non Cav. (1788)

Ind. loc.: "Geranium pilosum. Forst. prod. n. 531... Habitat in Nova Zeelandia. (v. s.)" Typus: Nova Zealandia, Forster 531 [lectotype, designated by CAROLIN (1965: 351), K!]

Herbs perennial, $30-50 \mathrm{~cm}$ tall. Rootstock 8-9 mm diam., vertical, not tuberculate, turnip-shaped. Stem decumbent or ascending, usually few per caudex branch, with patent to retrorse, not appressed, eglandular hairs 0.3-0.7 mm long. Basal leaves in a \pm deciduous rosette; lamina $1.6-2.5 \times 2-3 \mathrm{~cm}$, polygonal in outline, cordate, palmatifid (divided for $0.75-0.8$ of its length), pilose, with \pm erect, eglandular hairs; segments $5(-7)$, rhombic, $2-3 \mathrm{~mm}$ at the base, 3-4-lobed in distal half (ratio main-sinus length of the middle segment $/$ middle-segment length $=$ 0.14-0.2); cauline leaves opposite; petioles to $5 \mathrm{~cm}$ long, with patent, eglandular hairs 0.2-0.6 mm long; stipules 5-6 × 1-2 mm, lanceolate, with eglandular hairs on abaxial surface and on the margin, glabrous adaxially. Inflorescence a monochasial cyme; cymules 2-flowered, solitary; peduncles 1.1$3.3 \mathrm{~cm}$ long, with patent to retrorse, not appressed, eglandular hairs $0.2-0.5 \mathrm{~mm}$ long; bracteoles $2-2.5 \times 0.5-0.8 \mathrm{~mm}$, lanceolate, with eglandular hairs on abaxial surface and on the margin, glabrous adaxially; pedicels $0.5-1.5 \mathrm{~cm}$ long, with patent to retrorse, not appressed, eglandular hairs $0.2-0.5 \mathrm{~mm}$ long; pedicel and peduncle together overtopping the subtending leaf. Sepals $2.5-4 \times 2$ $2.3 \mathrm{~mm}$ (ratio pedicel length $/$ sepal length $=$ 
1.4-6), smooth, accrescent, 3-nerved, with mucro 0.4-0.5 mm long, with scarious margins $0.1 \mathrm{~mm}$ wide, with eglandular hairs 0.1 mm long on the abaxial side (and eglandular hairs 0.4-0.8 $\mathrm{mm}$ long on the margin), glabrous adaxially, ending in 1-3 bristles 0.3-0.4 mm long. Petals 5-12 $\times 2-5 \mathrm{~mm}$ (ratio petal length $/$ petal width $=2-2.4$ ), erectpatent, entire, without claw, glabrous on both sides, ciliate on the basal margin, purplish. Stamens 10, both whorls bearing anthers; filaments $2.2-2.7 \mathrm{~mm}$ long, not exserted, lanceolate, glabrous on both sides, ciliate on the basal margin, with hairs up to 0.1-0.3 $\mathrm{mm}$ long; anthers $0.4 \times 0.4 \mathrm{~mm}$. Nectaries glabrous. Gynoecium $4 \mathrm{~mm}$ long. Fruit 13$16 \mathrm{~mm}$ long, erect; mericarps $2.4-2.7 \times 1.1$ $1.2 \mathrm{~mm}$, without a strand of fibres, smooth, without longitudinal rib, without basal beak, with a basal callus, with erect-patent, eglandular hairs 0.1-0.6 mm long, usually blackish; rostrum 9-11 mm long, without a narrowed apex, with erect-patent, eglandular hairs 0.1-0.5 mm long; stigmatic remains
0.7-0.9 mm long (ratio fruit length / stigmatic remains length $=14-18$ ), with 5 hairy lobes. Seeds 1.5-1.6 $\times 0.9-1 \mathrm{~mm}$, reticulate, brownish; hilum 1/6 as long as the perimeter. Cotyledons entire.

Flowering May-June. Weedy areas and roadsides; 0-100 m. Australia \& New Zealand; introduced in Southwestern U.S.A. Fig. 19.

Illustrations. Fig. 14f. HARDEN (1992: 25).

TAYLOR (1993: 674) includes "Geranium pilosum Forster f." as a synonym of $G$. potentilloides and does not accept $G$. solanderi in California, suggesting a misunderstanding between both species. Geranium potentilloides differs from $G$. solanderi in its 1-flowered cymules, not turnip-shaped rootstock, obtriangular leaf segments, and longer sepals.

\section{Representative specimens examined}

USA. California: Humboldt Co., Trinidad, $41^{\circ} 3^{\prime} \mathrm{N}$, 124ㅇ' W, 23-V-1931, Tracy 9453 (UC), 11-VI-1911, Tracy 3238 (UC).

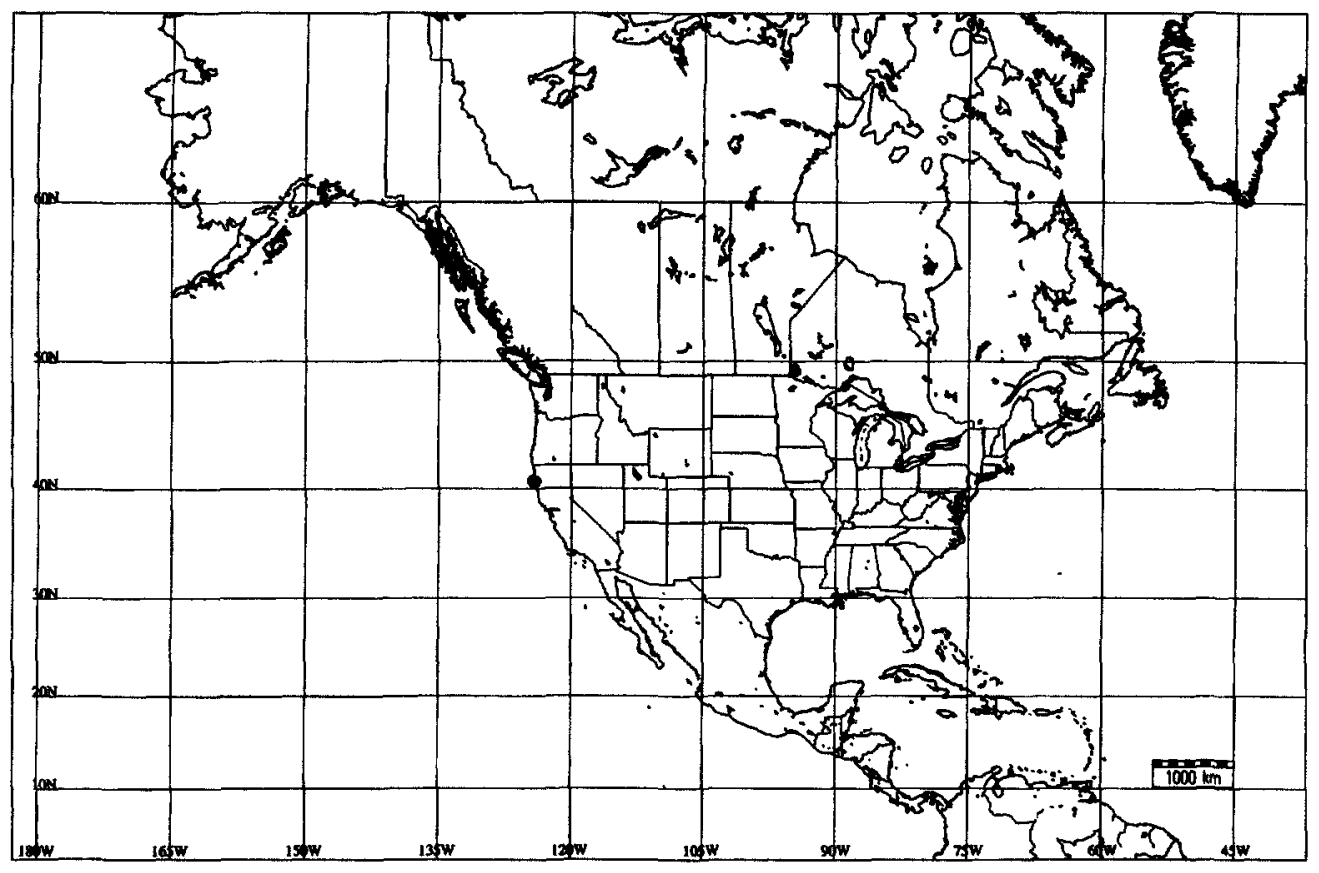

Fig. 19.-Distribution of Geranium solanderi in North America. 
12. Geranium caespitosum E. James, Account Exped. Pittsburgh ed. Amer. 2: 3 (1823)

G. intermedium E. James, Account Exped. Pittsburgh Eng. ed. 2: 344, 190 (1823), nom. illeg.

Ind. loc.: "About the sandstones ledges we collected a geranium..." ["the base of the Rocky Mountains"]

Typus: USA. New Mexico, San Miguel Co., Mora river, 18 Aug. 1847, Fendler 89 [neotype designated by JONES \& JONES (1943: 49), GH; isotypes, MO! UC F! P! W!]

= G. pentagynum Engelm. in Wisl., Mem. Tour. N. Mexico: 90 (1848)

Ind. loc.: "On Wolf creek, flowers in June"

Typus: USA. New Mexico, Wolf Creek, Wislizenus 508 (lectotype, here designated, MO!)

$=$ G. fremontii Torr. ex A. Gray, Pl. Fendler.: 26 (1849)

G. caespitosum var. fremontii (Torr. ex A. Gray) Dorn, Vasc. Pl. Wyoming: 180, 304 (1988), nom. inval., without basionym

Ind. loc.: "G. Fremontii (Torr.! in Pl. Frem. cum ic. ined.)... Bottom lands of the Mora River, among shrubs; August. (Also in the Raton Mountains, Lieut. Abert, and probably farther north and west by Col. Fremont.)"

Typus: USA. New Mexico, Raton Mountains, 7 Aug 1846, Abert s.n. [lectotype, designated by JONES \& JONES (1943: 45), NY!; isolectotype, $\mathrm{GH}$ !]

$=$ G. gracile Engelm. in A. Gray, Pl. Fendler.: 27 (1849), nom. illeg., non Ledeb. ex Nordm., 1837; publ. in Mem.

Acad. Arts Sci. ser. 21: 1-116

G. caespitosum var. gracile Engelm. ex A. Nelson \& J.F. Macbr. in Bot. Gaz. (Crawfordsville) 55: 376 (1913)

Ind. loc.: "Pine woods, on the mountains of Cosiquiriachi, State of Chihuahua; collected in October, by Dr. Wislizenus"

Typus: Mexico. Chihuahua, Cosiquiriachi, Wislizenus s.n. (no authentic material located )

$=$ G. fremontii var. parryi Engelm. in A. Gray in Amer. J. Sci. Arts ser. 2, 33: 405 (1862)
G. parryi (Engelm.) A. Heller, Cat. N. Amer. Pl. ed. 2: 7 (1900)

G. caespitosum var. parryi (Engelm.) W.A. Weber in Phytologia 33: 105 (1976)

Ind. loc.: [plants of Dr. Parry's Collection in the Rocky Mountains in 1861, n. 113] ["head-waters of Clear Creek, and the alpine ridges lying east of Middle Park, Colorado Territory" in MO label]

Typus: USA. Colorado, Clear Creek, Middle Park, 1861, Parry 113 [lectotype, designated by JONES \& JONES (1943: 43), NY; isolectotypes, $\mathrm{GH} \mathrm{K}$ ! MO! P!]

$=$ G. caespitosum f. albiflorum Cockerell in Sci.-Gossip. 25: 188 (1889)

Ind. loc.: "Looking over the herbarium of Mrs. M.E. Cusack, I find several things of interest... All the above-mentioned plants were collected in Western Custer Co., Colorado" Typus: USA. Colorado, Western Custer Co., M.E. Cusack s.n. (no authentic material located)

$=G$. richardsonii var. intermedium Kuntze, Revis. Gen. Pl. 1: 93 (1891)

Ind. loc.: "Colorado, Rocky Mountains"

Typus: USA. Colorado, Rocky Mountains, 20 Sept. 1874, O. Kuntze 3024 (lectotype, here designated, NY!)

$=$ G. atropurpureum A. Heller in Bull. Torrey Bot. Club 25: 195 (1898)

G. caespitosum subsp. atropurpureum (A. Heller) W.A. Weber in Phytologia 53: 187 (1983)

Ind. loc.: "The description is drawn from our no. 2723, collected along Santa Fé Creek, June to July, 1897. It is very plentyful along the stream, but always in dry ground. It has a range of about 2000 feet, as it was first collected four milles east of Santa Fé, at an elevation of a little over 7000 feet, but later was seen growing on a slope ten milles up the valley, and at an elevation of almost 9000 feet. It was also noticed along the road between Santa Fé and Cañoncito"

Typus: USA. New Mexico, Santa Fé Creek, 17 July 1897, Heller 3723 (lectotype, here designated, MO!; isolectotypes, BM! GH ILL LE! K! NY P!) [number collection referred in the protologue is 2723 , not 3723 , which is a probably mistake] 
$=$ G. pattersoni Rydb. in Bull. Torrey Bot. Club 29: 242 (1902)

Ind. loc.: "Colorado: Gray Peak, 1895, P.A. Rydberg (type in herb. N.Y. Bot. Gard.)" Typus: USA. Colorado, Gray Peak, 1895, Rydberg s.n. [lectotype designated by KNUTH (1912: 100), NY!]

$=$ G. cowenii $\mathrm{Rydb} .$, Fl. Colorado: 218 (1906) G. fremontii var. cowenii (Rydb.) H.D. Harr., Man. Pl. Colorado: 641 (1954) G. atropurpureum var. cowenii (Rydb.) Dorn, Vasc. Pl. Wyoming: 180, 304 (1988), nom. inval., without basionym

Ind. loc.: "In the mountains of Colo.-Alt. 6000-7000 ft.-Hills, Larimer Co.; Horsetooth Gulch; Rist Cañon; La Veta"

Typus: USA. Colorado, Larimer Co., Rist Cañon, W.F. Marshall 1157 (lectotype, here designated, NY!) [40 $38^{\circ} \mathrm{N}, 105^{\circ} 11^{\prime} \mathrm{W}$ ]

$=$ G. furcatum Hanks in Underw. \& Britton (eds.), N. Amer. Fl. 25(1): 16 (1907)

G. atropurpureum var. furcatum (Hanks) Kearney \& Peebles in J. Wash. Acad. Sci 29: 485 (1939)

Ind. loc.: "Type collected in the Grand Cañon of the Colorado, Arizona, August, 1897, T. F. Allen"

Typus: USA. Arizona, Grand Cañon of the Colorado, Aug. 1897, T. F. Allen s.n. [lectotype, designated by JONES \& JONES (1943: 46), NY!; isotype, UC]

$=$ G. marginale Rydb. ex Hanks \& Small in Underw. \& Britton (eds.), N. Amer. Fl. 25(1): 16 (1907)

Ind. loc.: "Type collected on the Aquarius Plateau at the head of Poison Creek, Utah, August 4, 1905, P. A. Rydberg \& E. C. Carlton 7401"

Typus: USA. Utah, Aquarius Plateau at the head of Poison Creek, 4 Aug. 1905, P. A. Rydberg \& E. C. Carlton 7401 (lectotype, here designated, NY!)

$=$ G. eremophilum Wooton \& Standl. in Contr. U.S. Natl. Herb. 16(4): 142 (1913)

G. caespitosum var. eremophilum (Wooton \& Standl.) W.C. Martin \& C.R. Hutchins, Fl. New Mexico 1: 1121 (1980)

Ind. loc.: "Type in the U.S. National Herbarium, no. 233003 , collected in the
San Luis Mountains, September 5, 1893, by Dr. E. A. Mearns (no. 2142)"

Typus: USA. New Mexico, San Luis Mountains, 5 Sep. 1893, Mearns 2142 (holotype, US-233003!)

= G. toquimense A.H. Holmgren \& N.H. Holmgren in Brittonia 26: 311, fig. 2 (1974)

Ind. loc.: "UNITED STATES: Nevada: Nye County, Toquima Range, head of the $\mathrm{N}$ branch of South Fork Pine Creek, T. 11 N., R. 45 E., Sec. 21 , elev. $3150 \mathrm{~m}(10,500$ ft), 3 Aug 1964, N. Holmgren \& Reveal $1518^{\prime \prime}$

Typus: USA. Nevada, Nye Co., Toquima Range, head of the $\mathrm{N}$ branch of South Fork Pine Creek, 3 Aug. 1964, N. Holmgren \& Reveal 1518 (holotype, NY!; isotypes, BRY DAO ID RSA UC UT UTC WTU)

Herbs perennial, $15-70 \mathrm{~cm}$ tall. Rootstock 5$14 \mathrm{~mm}$ diam., vertical, not tuberculate, not turnip-shaped. Stem erect to ascending, usually few per caudex branch, with patent to retrorse, eglandular hairs $0.1-0.8 \mathrm{~mm}$ long, and often patent, glandular hairs $0.2-1.1 \mathrm{~mm}$ long. Basal leaves in a persistent rosette; lamina 2.8$7.1 \times 3-7.5 \mathrm{~cm}$, polygonal in outline, cordate, palmatifid (divided for $0.72-0.88$ of its length), pilose, with appressed, eglandular (and sometimes glandular) hairs; segments 5(-7), obtriangular to rhombic, 4-10 $\mathrm{mm}$ at the base, 3-7lobed in distal half (ratio main-sinus length of the middle segment $/$ middle-segment length $=$ $0.1-0.25$ ); cauline leaves opposite; petioles to $30 \mathrm{~cm}$ long, with patent to retrorse, eglandular hairs 0.1-0.9 mm long, and often patent, glandular hairs 0.1-1.1 mm long; stipules 7-11 $\times$ 1.5-2 $\mathrm{mm}$, lanceolate, with eglandular (and sometimes glandular) hairs on abaxial surface and on the margin, glabrous adaxially. Inflorescence a dichasial cyme; cymules 2-flowered, solitary or rarely in aggregates at the top of each branch; peduncles (0-)1.8-12 cm long, pilose, with patent, glandular hairs $0.2-0.6 \mathrm{~mm}$ long and patent to retrorse, eglandular hairs 0.2-0.7 mm long, sometimes lacking glandular hairs; bracteoles 6-11 $\times 1-1.5 \mathrm{~mm}$, linear, with eglandular hairs on both sides and and on the 


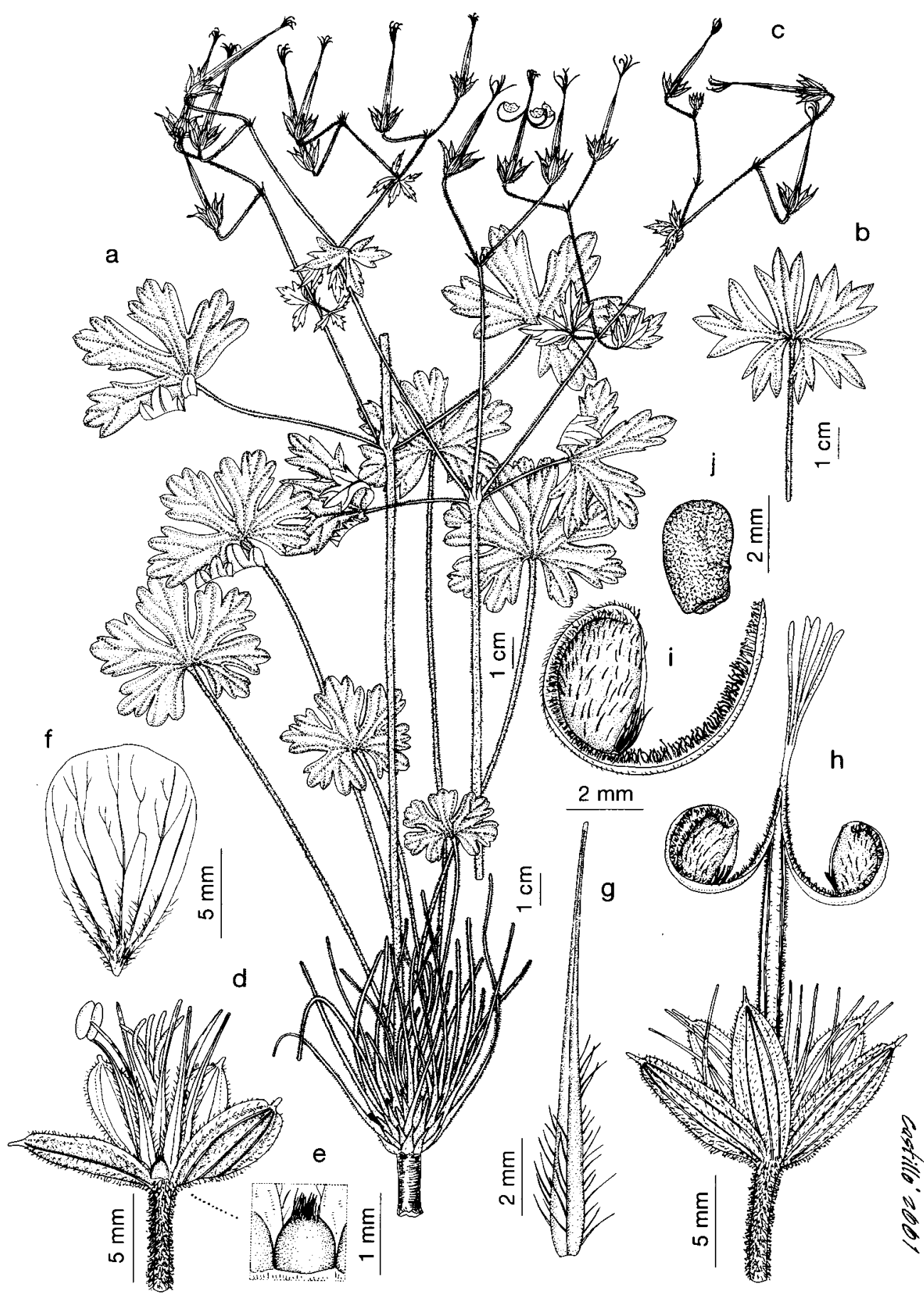

Fig. 20.-Geranium caespitosum: a, Habit; b, Leaf; c, Inflorescence; d, Flower without petals; e, Nectary; f, Petal; g, Staminal filament; h, Fruit; i, Mericarp; j, seed. [a, f-j based on Aedo 4424 (MA), b-e based on Hogan 2272 (MA)]. 
margin; pedicels $1.8-4 \mathrm{~cm}$ long, with glandular, patent hairs $0.2-0.7 \mathrm{~mm}$ long and eglandular, patent to retrorse hairs $0.2-0.7 \mathrm{~mm}$ long, sometimes lacking glandular hairs; pedicel and peduncle together often overtopping the subtending leaf. Sepals 8-11 $\times 3-4 \mathrm{~mm}$ (ratio pedicel length / sepal length $=2-5.1$ ), smooth, not accrescent, 3-5-nerved, with mucro (0.5-) 1-2 mm long, with scarious margins $0.2-0.3$ mm wide, with eglandular, antrorse to patent, hairs $0.2-0.9 \mathrm{~mm}$ long and glandular, patent hairs 0.1-0.6 mm long on the abaxial side, usually hairy on the base of the adaxial side. Petals $11-15 \times 6-9 \mathrm{~mm}$ (ratio petal length/ petal width $=1.6-1.8$ ), patent, entire, without claw, hairy on 1/3-1/2 of their adaxial surface and on the base of the abaxial one, ciliate on the basal margin, purplish, sometimes white. Stamens 10 , both whorls bearing anthers; filaments 8-9 mm long, not exserted, lanceolate, pilose on the abaxial side, ciliate on the proximal half, with hairs up to $1.5 \mathrm{~mm}$ long; anthers $2.5-3 \times 1$ $\mathrm{mm}$. Nectaries with a tuft of hairs at the top, dorsally glabrous. Gynoecium 10-13 $\mathrm{mm}$ long.
Fruit 22-31 (-33) mm long, erect when immature; mericarps $3-3.5 \times 2-2.5 \mathrm{~mm}$, without a strand of fibres, smooth, with longitudinal rib, without basal beak, with a basal callus, with eglandular, \pm patent hairs $0.2-0.8 \mathrm{~mm}$ long, and glandular 0.2-0.5 mm long, sometimes lacking glandular ones, brownish; rostrum 15-22 mm long, with a narrowed apex $2-4 \mathrm{~mm}$ long, with eglandular, \pm patent hairs 0.1-0.2 mm long, and glandular 0.5-0.6 mm long, sometimes lacking glandular ones; stigmatic remains 6-8 $\mathrm{mm}$ long (ratio fruit length $/$ stigmatic remains length $=$ $3.8-5.5$ ), with 5 glabrous lobes. Seeds $3.5 \times 2.5$ $\mathrm{mm}$, reticulate, brownish; hilum $1 / 4$ as long as the perimeter. Cotyledons entire. $n=26$.

Flowering May-October. Coniferous forests (also in aspen or oak woods), meadows, or disturbed roadsides; 1400-3300 m. Northwestern U.S.A., Southwestern U.S.A., South-Central U.S.A. \& North Mexico. Fig. 21.

Illustrations. Fig. 1f, 5c, 20.

This species shares with $G$. californicum, G. lentum, $G$. richardsonii, $G$. viscossisimum,

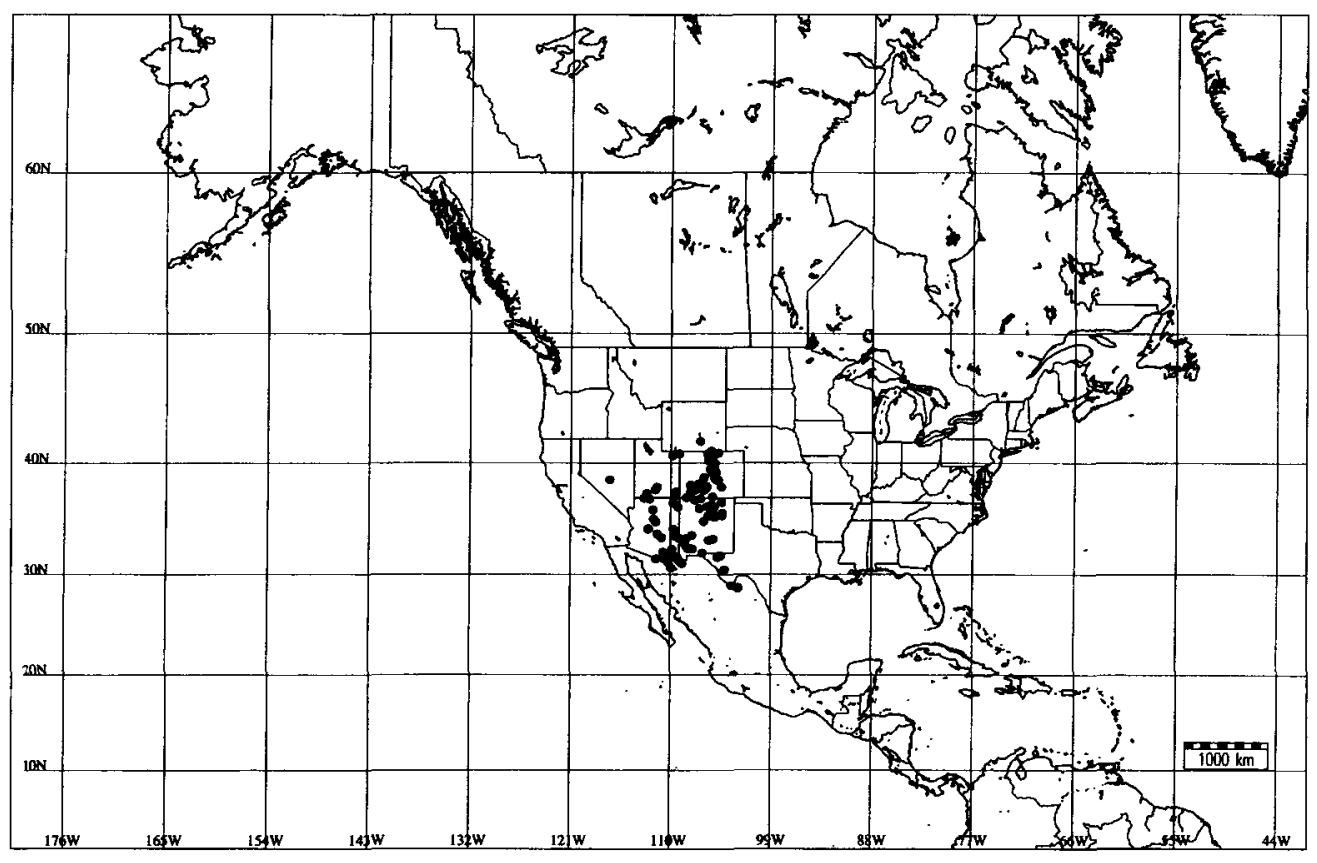

Fig. 21.-Distribution of Geranium caespitosum. 
and $G$. wislizenii petals with long hairs on adaxial surface. This feature is also found in other Mexican species not included in this study. KNUTH (1912: 45) described sect. Caespitosa, and typified it on G. caespitosum. An extension of Knuth's concept of sect. Caespitosa should be considered to include at least G. californicum, G. lentum, G. richardsonii, G. viscossisimum, and G. wislizenii, which probably constitute a natural group.

My treatment of $G$. caespitosum differs from those of KNUTH (1912) and JONES \& JONES (1943). I agree with MOORE (1943: 47) that $G$. caespitosum was unnecessarily divided into several species by those authors. Moreover, WELSH \& al. (1987:313) pointed out that separation of $G$. caespitosum in several taxa is possible only arbitrarily. Geranium caespitosum has a great variability in distribution and density of glandular indumentum, even in the same specimen. Although G. caespitosum has essentially eglandular indumentum, occasionally specimens occur with eglandular hairs on stem, petioles or inflorescence. Furthermore, leaf shape and proportion of petal surface bearing hairs varies considerably, but independently of glandular indumentum presence. Consequently these forms are here not accorded taxonomic recognition (Table 1).

In $G$. caespitosum glandular hairs are similar to those of $G$. oreganum, $G$. richardsonii or G. viscosissimum: shorter than in G. maculatum and ending in a broad usually yellow cell.

Geranium caespitosum could be confused with $G$. viscosissimum, which has greater leaves, stem, and fruits and shorter stigmatic remains.
I have considered as prioritary the American edition of Account of an expedition from Pittsburgh to the Rocky mountains... (JAMES 1823a) over the English edition (JAMES 1823b) in order to maintain nomenclatural stability. The converse option implies that $G$. caespitosum, unquestioned for more than 150 years, must be displaced by $G$. intermedium $E$. James.

\section{Representative specimens examined}

Mexico. Mexico Northeast: Coahuila, Gobbler Canyon, 28 $57^{\prime}$, 102 $^{\circ} 34^{\prime}$, 30-VIII-1997, Wood \& al. s.n. (UNM); Sierra del Jardín, Rancho El Caballo, 29 $3^{\circ}$, $102^{\circ} 37^{\prime}, 16-$-IX-1972, Chiang \& al. 9340 (MEXU); Sierra Maderas del Carmen, Campo El Tres, 29 $0^{\prime}, 102^{\circ} 36^{\prime}$, 9-X-1992, Wendt \& Adamcewicz 513 (MEXU). MEXICO Northwest: SonORA, Cerro de las Flores, $30^{\circ} 56^{\circ}$, $109^{\circ} 57^{\prime}, 9-\mathrm{X}-1992$, Fishbein \& al. 666 (MEXU).

USA. ARIzONA: Apache Co., Little Colorado River, 15 $\mathrm{mi}$ S of the junction of Arizona Highway 260, along Arizona Highway 273 around bridge over river, $3 \mathrm{mi} \mathrm{W}$ of Eager,

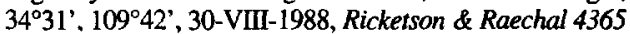
(MO); Cochise Co., Chiricahua Mountains, Barfoot Park, $31^{\circ} 54^{\prime}, 109^{\circ} 17^{\prime}, 13-1 X-1906$, Blumer 1370 (NMC); Cochise Co., Huachuca Mountains, Miller Canyon, $31^{\circ} 25^{\prime}$, 110 14', 9-VIII-1980, Carter s.n. (NMC); Coconino Co., Kendrick Peak, along Trail 22, 35 $24^{\prime}, 111^{\circ} 51^{\prime}, 5$-VIII1983, Ricketson 50I (MO); Gila Co., Barnhardt Pass, Matzatzal Mountains, $34^{\circ} 4^{\prime}, 111^{\circ} 28^{\prime}$, Collom 111 (MO); Graham Co., Shannon Camp, Pinaleno Mountains, 32 $39^{\prime}$, 10951', 3-IX-1944, Darrow \& al. 1060 (MO); Pima Co., Baboquivari Mts., Baboquivari Canyon, $31^{\circ} 47^{\prime}, 111^{\circ} 37^{\prime}, 4-$ IX-1931, Gilman 21 (MO); Santa Cruz Co., cañon in Santa Rita Mountains, $31^{\circ} 49^{\prime}, 110^{\circ} 46^{\circ}, 26-[X-1880$, Engelmann s.n. (MO); White Mountains, $10 \mathrm{mi}$ W of McNary, 33⒌', 109'35', 23-VI-1930, Goodman \& Hitchock 1328 (MO); Yavapai Co., Prescott. $34^{\circ} 32^{\prime}, 112^{\circ} 28^{\prime}, 15$-VII-1896, Luck s.n. (MO). Colorado: Archuleta Co., Sheep Cañon, $37^{\circ} 8^{\prime}$, 107 24', 11-VII-1901, Clements \& Clements 90 (MO). COLORADO: Boulder Co., Boulder Falls, 40 0', 105 $24^{\prime}$, -VIII-, Graves 2219 (MO); Clear Creek Co., top of Douglas' Mountain, Empire, $39^{\circ} 45^{\prime}, 105^{\circ} 40^{\prime}$, -VIII-, Engelmann s.n. (MO); Costilla Co., $8 \mathrm{mi}$ NE Fort Garland, on Hwy 160,

\section{TABLE 1}

DISTRIBUTION OF INDUMENTUM ON GERANIUM CAESPITOSUM AND RELATED SPECIES ACCORDING TO JONES \& JONES (1943)

\begin{tabular}{|l|c|c|c|c|}
\hline & $\begin{array}{c}\text { Petal surface } \\
\text { bearing hairs }\end{array}$ & $\begin{array}{c}\text { Glandular hairs } \\
\text { on pedicels }\end{array}$ & $\begin{array}{c}\text { Glandular hairs } \\
\text { on petioles }\end{array}$ & $\begin{array}{c}\text { Glandular hairs } \\
\text { on stem base }\end{array}$ \\
\hline G. caespitosum & $1 / 3$ & - & - & - \\
\hline G. eremophilum & $1 / 2$ & + & - & - \\
\hline G. fremontii & $1 / 4$ & + & - & - \\
\hline G. parryi & $1 / 4$ & + & + & + \\
\hline G. marginale & $1 / 4$ & - & - & - \\
\hline G. cowenii & $1 / 4-1 / 3$ & - & - & - \\
\hline
\end{tabular}


37²5', 105²6', 23-VI-1966. Croat 2041 (MO); Custer Co., near St. Charles River, Santa Isabel City, $38^{\circ} 15^{\prime}$, 104²7', 23-VI-1936, Rollins 1222 (MO); Douglas Co., Waterton, 39 $29^{\prime}, 105^{\circ} 4^{\prime}, 2$-VIII-1921, Osterhout \& Clokey 4196 (MO); El Paso Co., near Manitou, 38 52', 10459', 23 VI-1926, Palmer 31248 (MO); Gilpin Co., Rollinsville, $39^{\circ} 55^{\circ}, 105^{\circ} 30^{\prime}, 8$-VII-1913, Overholte s.n. (MO); Hinsdale Co., near Henson Creek, $1 \mathrm{mi}$ SW of Lake City, $38^{\circ} \mathrm{l}^{\prime}$. 107'18', 14-VII-1951, Rollins 51105 (MO); Jefferson Co., US 285, $8.1 \mathrm{~km}$ E of Conifer. 39 $31^{\prime}, 105^{\circ} 18^{\prime}, 4$-VII-1977. Semple \& Brammall 2713 (MO); Lake Co., Twin Lakes, 39 4', 106 22', 16-VII-1919, Clokey 3584 (MO); Larimer Co., Sand Creek Pass, $40^{\circ} 53^{\prime}, 105^{\circ} 49^{\prime}, 3$-VIII-1897, Baker s.n. (MO): Montezuma Co., Mancos, $37^{\circ} 20^{\circ}, 108^{\circ} 17^{\prime}, 8-$ VII-1898, Baker \& al. 407 (MO NMC); Montrose Co., near Horsefly Creek, $38^{\circ} 25^{\prime}, 107^{\circ} 51^{\prime}, 6-$ VII-1924, Payson \& Payson 3908 (MO); Park Co., 2 mi S of US 285 at Pine Junction to Wandcrest Park. $39^{\circ} 26^{\prime}, 105^{\circ} 23^{\prime}, 27-$ VI-1971, Shea \& Shea 57 (MO); Saguache Co., Ute Pass, $38^{\circ} 10^{\prime}, 106^{\circ} 7^{\prime}$, 3-VII-1886 (MO); San Miguel Co., Ophir, 37 $51^{\prime}, 1^{\circ} 7^{\circ} 9^{\prime}$, 11-IX-1947, Weber 3590 (NMC); Teller Co., Highway 24, $1 \mathrm{mi}$ W of Divide, 38 $56^{\prime} .105^{\circ} 9^{\prime}$. 25-VII-1977, Dörr 553 (MO). Nevada: Nye Co., Toquima Range, Pine Creek, 38 $52^{\prime}, 116^{\circ} 45^{\prime}, 3$-VIII-1964, Holmgren \& Reveal 1518 (NY). NEw MExico: Bemalillo Co., Sandia Mountains, Balsam Park, 3512'. 106 24', 15-VIII-1914, Ellis 109 (MO); Catron Co., Mogollon Mts., $33^{\circ} 13^{\prime}, 108^{\circ} 31^{\prime}$, - VII-, Rusby 58 (MO): Colfax Co., Eagle Nest Lake, $36^{\circ} 32^{\prime}, 105^{\circ} 14^{\prime}, 6-$ VII-1937, Schwarz \& Talley s.n. (MO); Dona Ana Co., Organ Mountains, Aguirre Springs Recreation Area, $32^{\circ} 19^{\prime}$, 106 33', 11-IX-1947, Weber 3590 (NMC); Eddy Co., Guadalupe Mountains, Devils Den Canyon, $32^{\circ} 2^{\prime}, 104^{\circ} 49^{\prime}$, 6-IX-1986, Brunt 16 (NMC); Grant Co., Burro Mountains, $32^{\circ} 36^{\prime}, 10^{\circ} 26^{\prime}, 20$-VI-1903, Metcalfe 194 (MO); Harding Co., Whitman Camp, 35 $57^{\prime}, 104^{\circ} 20^{\prime}$, 9-IX-1895. Mulford 1243 (MO); Hidalgo Co., Peloncillo Mountains, Skeleton Canyon, 31 $37^{\circ}, 109^{\circ} 5^{\prime}, 6$ - IX-1981, Spellenberg \& Spellenberg 6324 (NMC); Lincoln Co., White Mountains, 3329', $105^{\circ} 50^{\circ}$. 27-VII-1897, Wooton 218(MO); Mora Co., Rte. 3, 6.3 mi E of Taos Co. line, $36^{\circ} 2^{\prime}, 15^{\circ} 22^{\prime}$. 12-VIII-1984, Hill 15103 (MO); Rio Arriba Co., Wolf Creek, Castel Mountains, $36^{\circ} 22^{\prime}, 106^{\circ} 51^{\circ}, 24-V_{1}-1846$, Wislizenus 508 (MO); San Miguel Co., Las Vegas, $35^{\circ} 35^{\prime}, 105^{\circ} 13^{\prime}, 12$-LX-1881, Engelmann s.n. (MO): Sierra Co., Taylor Creek. 32 $42^{\circ}$, 107³8', 14-VIII-1982, Spellenberg \& al. 6589 (NMC); Socorro Co., San mateo Mountains, Sargent Canyon, 33 ${ }^{\circ} 56^{\circ}$, $107^{\circ} 41^{\prime}, 23-V I I I-1978$, Moir \& Fitzhugh 670 (NMC); Taos Co., Cabresto Creek, $36^{\circ} 41^{\prime}$, $105^{\circ} 35^{\circ}$, 21-VII-1973, Moir 99 (NMC). Texas: Brewster Co., Chisos Mts., 29${ }^{\circ} 15^{\prime}$, 10318', 27-VII-1931, Mueller 8050 (F, TEX); Culberson Co., Guadalupe Mts., Pine Canyon, 31 $54^{\prime}, 104^{\circ} 52^{\prime}, 5$-IX1916, Young s.n. (MO); Jeff Davis Co., Davis Mountains. 30 $45^{\prime}, 104^{\circ} 5^{\prime}$, 6-X-1926, Palmer 32011 (MO); Jeff Davis Co., Davis Mts., Livermore Peak, 30 37', 104 10', 9-VII1921, Ferris \& Duncan 2550 (MO). Utah: Daggett Co., vicinity of Flaming Gorge, Sheep Creek Canyon, 40 $0^{\circ} 4^{\prime}$, $109^{\circ} 49^{\circ}, 6$-VI-1932, Williams 550 (MO); Garfield Co., $6 \mathrm{mi}$ W of Panguitch Lake, $37^{\circ} 42^{\prime}, 112^{\circ} 38^{\prime}, 17$-VII-1930, Goodman \& Hitchcock I587 (MO); Kane Co., along Skutumpah Creek, $37^{\circ} 13^{\prime}, 112^{\circ} 21^{\prime}$, 4-VIII-1976, Welsh \& al. 14233 (MO); N slope of Abajo Mountains, 37 $50^{\prime}, 19^{\circ} 27^{\prime}, 1$-VII1930, Goodman \& Hitchcock 1450 (MO); S de Grover, Dix- ie National Forest, $38^{\circ} 15^{\prime}, 111^{\circ} 30^{\circ}$, 19-VIII-1997, Aedo 4419 (MA); San Juan Co., Bulldog Canyon. 37 $40^{\circ}$, 109 27', 1-VII-1932, Maguire \& Redd 1953 (MO); Washington Co., Zion National Park, Temple of Sinawava, $37^{\circ} 17^{\prime}, 112^{\circ} 56^{\circ}, 22$-VIII-1997, Aedo 4428 (MA). Wyoming: Albany Co.. Chimney Rock, Sand Creek. $41^{\circ} 12^{\prime}, 105^{\circ} 43^{\prime}, 29$-VII-1929, Greenman \& Greenman 6045 (MO); Albany Co., Jelm, 41 ${ }^{\circ} 3^{\prime}, 106^{\circ} 0^{\circ}, 11$ - VIII-1900, Nelson 8061 (MO); Horseshoe Creek, NW of Laramie, $4^{\circ} 5^{\circ}, 106^{\circ} 47^{\prime}$. 7-VIII-1858, Engelmann 186 (MO); Laramie Co., Cheyenne, $41^{\circ} 8^{\prime}, 104^{\circ} 49^{\prime}, 6$, 6III-1901, Nelson 8591 (MO); Laramie Co., Granite Cañon, $41^{\circ} 5^{\prime}, 105^{\circ} 3$ ', 6-VIII-1880, Earle s.n. (MO); Sweetwater Co., Telephone Canyon, 41 4', 1095'. 15-VI-1894, Nelson 233 (MO).

13.Geranium californicum G.N. Jones \& F.L. Jones in Rhodora 45: 38 (1943)

G. leucanthum Small in Underw. \& Britton (eds.), N. Amer. Fl. 25(1): 18 (1907) [syn. subst.], nom. illeg., non Griseb. (1874) Ind. loc.: "Type collected at Pineridge, Fresno County, California, June 15-25, 1900, H. M. Hall \& H. P. Chandler 224"

Typus: USA. California, Fresno Co., Pineridge, 15-25 June 1900, Hall \& Chandler 224 [lectotype, designated by JONES \& JONES (1943: 39), NY; isolectotype, MO! UC!]

$=$ G. concinnum G.N. Jones \& F.L. Jones in Rhodora 45: 36 (1943)

Ind. loc.: "California: Kern River, Culbertson 4454"

Typus: USA. California, Tulare Co., Kern River, 3 Aug. 1904, Culbertson 4454 (holotype, GH; isotypes, MO! NY UC!) $=G$. viscicum, nom. nud., in sched. (MO!)

Herbs perennial, $20-70 \mathrm{~cm}$ tall. Rootstock 5$15 \mathrm{~mm}$ diam., vertical, not tuberculate, not numip-shaped. Stem erect to ascending, usually few per caudex branch, with patent to retrorse, eglandular hairs $0.3-0.9 \mathrm{~mm}$ long, and often patent, glandular hairs $0.3-0.5 \mathrm{~mm}$ long. Basal leaves in a persistent rosette; lamina 3.2-4.8 $\mathrm{x}$ $4.1-6 \mathrm{~cm}$, polygonal in outline, cordate, palmatifid (divided for 0.7-0.81 of its length), pilose, with appressed, eglandular (and sometimes glandular) hairs; segments 5(-7), rhombic, 3-7 $\mathrm{mm}$ at the base, 7-9-lobed in distal half (ratio main-sinus length of the middle segment / middle-segment length $=0.18-0.35$ ); cauline leaves opposite; petioles to $15 \mathrm{~cm}$ long, with patent to retrorse. eglandular hairs $0.3-0.9 \mathrm{~mm}$ long, and 
sometimes patent, glandular hairs 0.3-0.5 mm long; stipules 7-9 $\times 1.5-2 \mathrm{~mm}$, lanceolate, with eglandular hairs on abaxial surface and on the margin, glabrous adaxially. Inflorescence a dichasial cyme; cymules 2-flowered, solitary or rarely in aggregates at the top of each branch; peduncles $2.2-24 \mathrm{~cm}$ long, pilose, with patent, glandular hairs $0.2-0.6 \mathrm{~mm}$ long and patent to retrorse, eglandular hairs 0.3-1 mm long; bracteoles 5-11 $\times$ 1-1.5 mm, linear, with eglandular hairs on both sides and and on the margin; pedicels $2.8-8 \mathrm{~cm}$ long, with glandular, patent hairs $0.2-0.6 \mathrm{~mm}$ long and eglandular, patent to retrorse hairs $0.3-1 \mathrm{~mm}$ long; pedicel and peduncle together often overtopping the subtending leaf. Sepals 8-11 $\times 3-4.5 \mathrm{~mm}$ (ratio pedicel length / sepal length $=3.1-8.8$ ), smooth, not accrescent, 3-5-nerved, with mucro 1-2 mm long, with scarious margins $0.2-0.3 \mathrm{~mm}$ wide, with eglandular, antrorse to patent, hairs $0.2-1 \mathrm{~mm}$ long and glandular, patent hairs $0.2-0.6 \mathrm{~mm}$ long on the abaxial side (sometimes lacking glandular ones), usually hairy on the base of the adaxial side. Petals 11-15 $\times 6-11 \mathrm{~mm}$ (ratio petal length / petal width $=1.3-1.8$ ), erect-patent, entire, without claw, hairy on 1/2-3/4 of their adaxial surface and on the base of the abaxial one, ciliate on the basal margin, white, sometines pinkish. Stamens 10, both whorls bearing anthers; filaments 7-9 mm long, not exserted, lanceolate, pilose on the abaxial side, ciliate on the proximal half, with hairs up to $1.1 \mathrm{~mm}$ long; anthers 2-2.5 $\times 1 \mathrm{~mm}$. Nectaries with a tuft of hairs at the top, dorsally glabrous. Gynoecium 11-14 mm long. Fruit 32-42 mm long, erect when immature; mericarps 4.5-5 $\times 2.5-3 \mathrm{~mm}$, without a strand of fibres, smooth (with 2 transversal veins at the apex), with longitudinal rib, without basal beak, with a basal callus, with eglandular, \pm patent hairs 0.2-1.1 mm long, and glandular 0.2-0.6 $\mathrm{mm}$ long, brownish; rostrum 21-23 mm long, with a narrowed apex 5-6 mm long, with eglandular, \pm patent hairs 0.1-0.2 mm long, and glandular 0.3-0.6 mm long; stigmatic remains 6-8 $\mathrm{mm}$ long (ratio fruit length / stigmatic remains length $=4.5-5.6$ ), with 5 usually hairy lobes. Seeds $2.8 \times 1.7 \mathrm{~mm}$, reticulate, brownish; hilum $1 / 4$ as long as the perimeter. Cotyledons entire.
Flowering April-August. Coniferous or oak forests, meadows, or damp places; 1100 $3000 \mathrm{~m}$. Southwestern U.S.A. Fig. 23.

\section{Illustrations. Fig. 22.}

Geranium californicum is quite similar to $G$. caespitosum. However, it differs in the longer fruits, longer narrowed apex of the rostrum, and usually white petals. Additionally, in most $G$. californicum specimens there are some longer pedicels towards the inflorescence base. It can be distinguished from $G$. viscossisimum by its narrow leaves and longer stigmatics remains. G. californicum always bears glandular hairs on the inflorescence, although there are specimens without these hairs on the stem base.

Geranium concinnum falls within the range of variation encompassed by my concept of $G$. californicum, which is in agreement with TAYLOR (1993: 673). JONES \& JONES (1943: 38) distinguished $G$. concinnum from $G$. californicum on the basis of the pubescence of pedicels and stem, and its shorter stigmatic remains (4.5-5.5 $\mathrm{mm}$ long). However, it is interesting to note that the type of $G$. concinnum has some stigmatic remains 6-7 mm long. Additionally, $G$. californicum shows a pubescence variation that includes that of $G$. concinnum.

A specimen from Clifton (Los Angeles Co.) [Davison 610, JEPS] has quite different (not deeply divided) leaves. In the rest of the characters, it does not differ from G. californicum.

\section{Representative specimens examined}

USA. California: Fresno Co., Pine Ridge, 36 $47^{\circ} \mathrm{N}$, $119^{\circ} 7^{\prime} \mathrm{W}, 15-\mathrm{VI}-1900$, Hall \& Chandler 224 (MO); Fresno Co., Shaver Lake, 37 $6^{\prime} \mathrm{N}, 119^{\circ} 19^{\prime} \mathrm{W}, 13-\mathrm{VHI}-1962$, Howe 3423 (SD); Kern Co.. Greenhorn Range, $35^{\circ} 44^{\prime} \mathrm{N}$, 118 33'W, 10-VI-1904, Hall \& Babcock 5048 (UC); Kem Co., Piute Mountains, French Meadow, 35 $27^{\prime} \mathrm{N}$, $18^{\circ} 20^{\prime} \mathrm{W}, 19-\mathrm{VII}-1962$. Twisselmann 7479 (MO); Los Angeles Co, Clifton, 3349' N, $11^{\circ} 22^{\prime} \mathrm{W}, 1-\mathrm{X}-1900$, Davison 610 (JEPS); Madera Co., North Fork, $37^{\circ} 13^{\prime} \mathrm{N}, 119^{\circ} 30^{\prime} \mathrm{W}$, 26-IV-1934, Bacigalupi 2273 (UC); Mariposa Co., Big Meadow, $37^{\circ} 42^{\prime} \mathrm{N}, 119^{\circ} 45^{\prime} \mathrm{W}, 6$-VII-1896, Jepson 13571 (JEPS); Mariposa Co., Gin Flat, $37^{\circ} 45^{\prime} \mathrm{N}, 19^{\circ} 46^{\prime} \mathrm{W}, 30-\mathrm{V}$ 1924, Jepson 10508 (JEPS); Mariposa Co., Ililouette, $37^{\circ} 42^{\prime} \mathrm{N}, 119^{\circ} 34^{\prime} \mathrm{W}, 24$ VII-1934, Plair 7(UC); Mariposa Co.. Stoneman, $37^{\circ} 44^{\prime}$ N, $119^{\circ} 34^{\prime} \mathrm{W}, 9-\mathrm{VI}-1911$, Abrams 4386 (UC); Mariposa Co., Yosemite National Park, Glacier Point, 3743'N, $119^{\circ} 34^{\prime} \mathrm{W}, 31-\mathrm{VII}-1941$, Wylie s.n. (SD); Mariposa Co., Yosemite Valley, 37 $44^{\prime} \mathrm{N}, 119^{\circ} 35^{\prime} \mathrm{W}, 17$ VII-1872, Redfield 42 (MO); San Bernardino Co., Holcomb Creek, $34^{\circ} 17^{\prime} \mathrm{N}, 117^{\circ} 7^{\prime} \mathrm{W}, 25-\mathrm{VII}-1986$, Sanders 


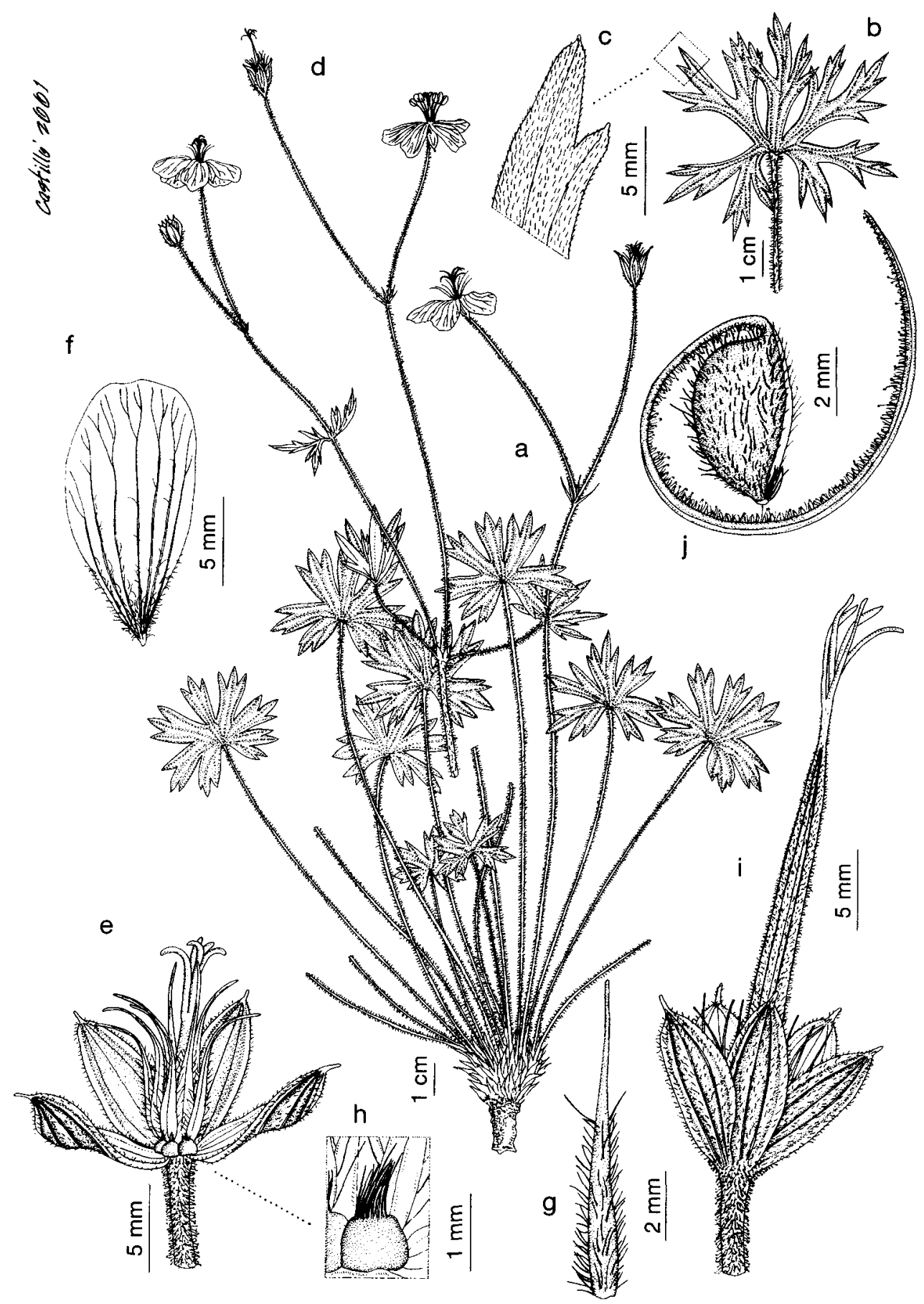

Fig. 22.-Geranium californicum: a, Habit; b, Leaf; c, Detail of leaf adaxial surface; d, Inflorescence; e, Flower without petals; f, Petal; g, Staminal filament; h, Nectary; i, Fruit; j, Mericarp. [a, d-h based on Hall \& Chandler 224 (UC), b-c based on Hall 9234 (UC), i-j based on Ertter 6665 (UC)]. 


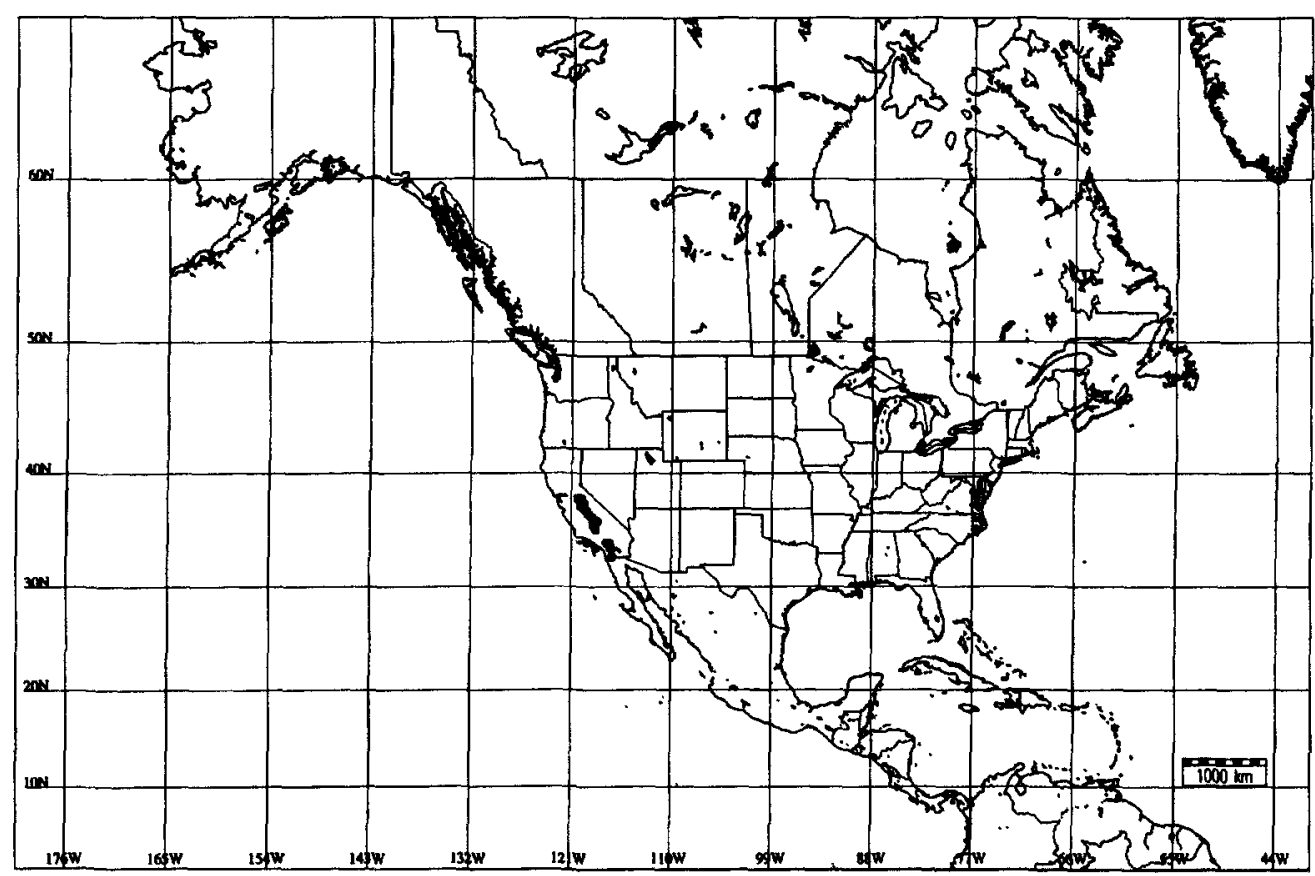

Fig. 23.-Distribution of Geranium californicum.

6783 (SD); San Bernardino Co., San Bernardino Mountains, Bluff Lake, eastern base, 34 $13^{\prime} \mathrm{N}, 116^{\circ} 58^{\prime} \mathrm{W}, 22$-VII-1894, Parish 3175 (MO); San Bernardino Mountains, Bear Valley, $34^{\circ} 15^{\prime} \mathrm{N}, 116^{\circ} 50^{\prime} \mathrm{W}, 19-\mathrm{VII}-1900$, Jones 6218 (MO); San Diego Co., Laguna mts., $33^{\circ} 0^{\prime} \mathrm{N}, 116^{\circ} 30^{\prime} \mathrm{W}$, -VII-, Orcutt 1507 (MO); San Diego Co., San Jacinto Mts., Tauquiz Meadow, $33^{\circ} 45^{\prime} \mathrm{N}, 116^{\circ} 40^{\prime} \mathrm{W}, 2-\mathrm{VII}-1892$, Hasse s.n. (MO); San Jacinto Mountains, vicinity of Deer Springs, $34^{\circ} 1^{\prime} \mathrm{N}, 116^{\circ} 47^{\prime} \mathrm{W},-\mathrm{VII}$, Hall 2336 (MO); Tulare Co., Kern River, 36 $18^{\prime} \mathrm{N}, 118^{\circ} 24^{\prime} \mathrm{W}, 3-\mathrm{VIII}-1904$. Culbertson 4454 (MO); Tulare Co., NW of Olancha Pass, Inyo NF ca $1.5 \mathrm{mi} \mathrm{SW}$ of Olancha, $36^{\circ} 12^{\prime} \mathrm{N}, 118^{\circ} 6^{\prime} \mathrm{W}, 8-\mathrm{VIII}-1986$, Ertter \& Shevock 6665 (MO UC); Tulare Co., Salmon Creek, 35 $53^{\circ}$ N, $118^{\circ} 28^{\prime}$ W, 17-VI-1904, Hall \& Babcock 5147 (UC); Tulare Co., Sequoia National Park, Camp Nelson, 368'N, $118^{\circ} 36^{\prime} \mathrm{W}, 26-\mathrm{VII}-1941$, Bacigalupi \& al. 2611 (UC); Tulare Co., Sequoia National Park, Cliff Creek, $36^{\circ} 32^{\prime} \mathrm{N}, 118^{\circ} 39^{\prime} \mathrm{W}, 31-\mathrm{VII}-1943$, Ferris \& Lorraine 10940 (UC); Tulare Co., Tule, $36^{\circ} 2^{\prime} \mathrm{N}, 118^{\circ} 19^{\prime} \mathrm{W}, 1897$. Purpus 5133 (MO); Tuolumne Co., Grohl Meadow, $38^{\circ} 12^{\prime} \mathrm{N}, 120^{\circ} 12^{\prime} \mathrm{W}, 3-\mathrm{VII}-1887$, Drew s.n. (UC); Tuolumne Co., Mather, 3752'N, $119^{\circ} 51^{\prime} \mathrm{W}, 2-\mathrm{VII}-1923$, Mason 2139 (UC); Tuolumne Co., Miguel Meadow, $37^{\circ} 57^{\prime} \mathrm{N}, 19^{\circ} 50^{\prime} \mathrm{W}, 8-\mathrm{VII}-1938$, Mason 11884 (UC); Tuolumne Co., Pine Crest Recreation Area. $38^{\circ} 11^{\prime} \mathrm{N}$. 119 59'W, 8-VI-1934, Wiggins 6810 (UC); Tuolumne Co. Reed Creek, 3757'N, $120^{\circ} 2^{\prime} \mathrm{W}, 20$-VIII-1948, Hinkley \& al. 1844 (UC); Tuolumne Co., Rosasco, $38^{\circ} 8^{\circ} \mathrm{N}, 119^{\circ} 49^{\prime} \mathrm{W}$, 25-VI-1889, Dunham s.n. (F); Tuolumne Co., Strawberry, $38^{\circ} 11^{\prime} \mathrm{N}, 120^{\circ} 0^{\prime} \mathrm{W}, 2$-VIII-1942, Rose 42215 (MO); Tuolumne Co., Twain Harte Post Office, $38^{\circ} 2^{\prime} \mathrm{N}$, $120^{\circ} 13^{\prime} \mathrm{W}, 30-\mathrm{V}-1944$, Alexander \& Kellogg 3589 (UC); Ventura Co., Grant Park, 34 17'N, $119^{\circ} 17^{\prime} \mathrm{W}, 13-\mathrm{VI}-1921$, Newlon 220 (JEPS).

14.Geranium lentum Wooton \& Standl. in Contr. U.S. Natl. Herb. 16(4): 142 (1913) Ind. loc.: "Type in the U.S. National Herbarium, no. 561079, collected on the West Fork of the Gila, August 7, 1900, by E. O. Wooton"

Typus: USA. New Mexico, Catron Co., West Fork of Gila, 7 Aug. 1900, Wooton s.n. [lectotype, designated by JONES \& JONES (1943: 51), US-561079!; isolectotype, UNM!] [33 $\left.10^{\prime} \mathrm{N}, 108^{\circ} 12^{\prime} \mathrm{W}\right]$

Herbs perennial, $35-50 \mathrm{~cm}$ tall. Rootstock ca. $10 \mathrm{~mm}$ diam., \pm horizontal, not tuberculate, not turnip-shaped. Stem erect, usually few per caudex branch, with patent, eglandular hairs $0.2-1 \mathrm{~mm}$ long, and often patent, glandular hairs 0.2-0.6 mm long. Basal leaves in a persistent rosette; lamina $2.8-5(-7) \times 3.4-$ $5.3(-7.5 \mathrm{~cm}$, polygonal in outline, cordate, palmatifid (divided for $0.65-0.8$ of its length), 


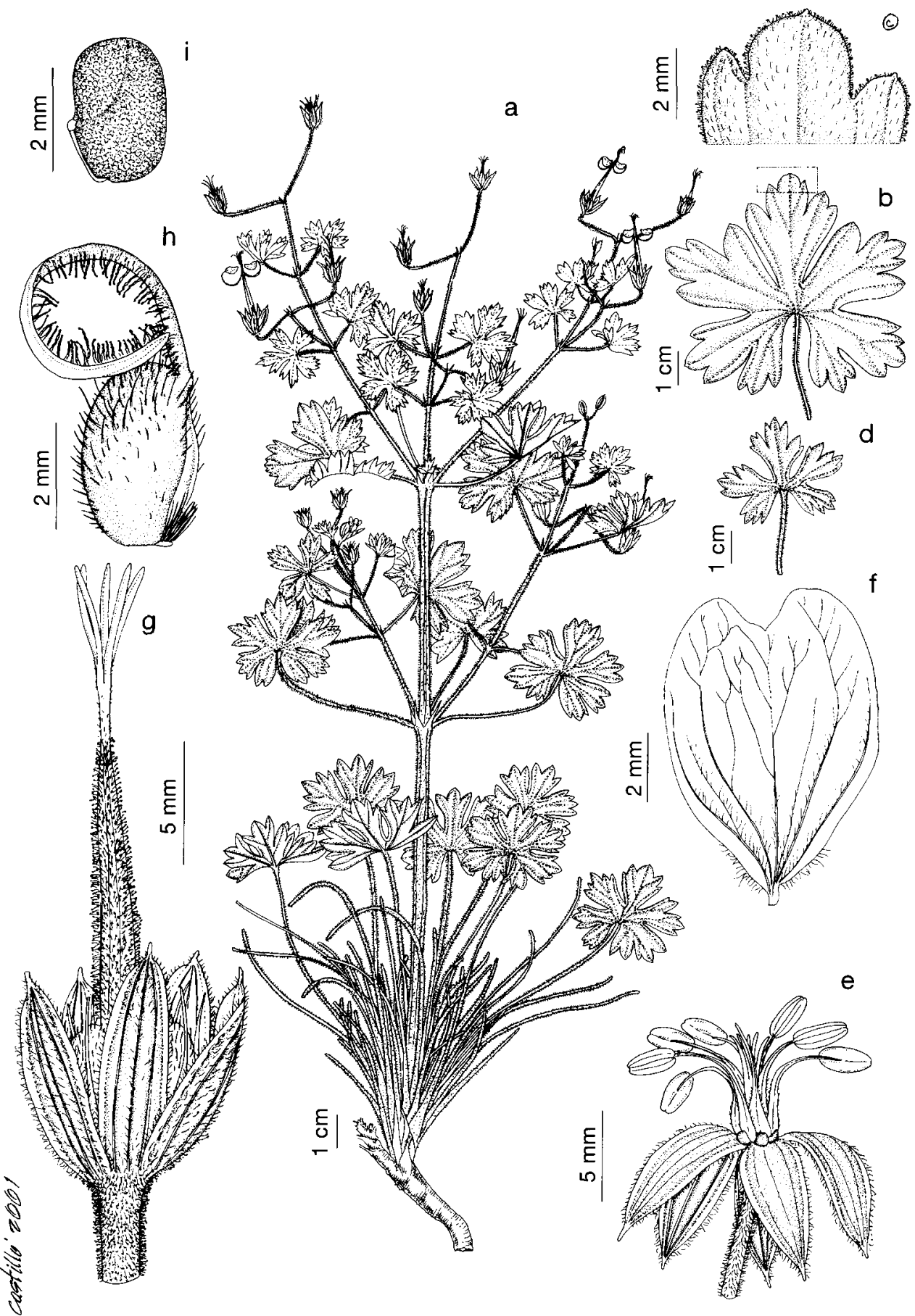

Fig. 24.-Geranium lentum: a, Habit; b, d, Leaves; c, Detail of leaf adaxial surface; e, Flower without petals; $f$, Petal. g, Fruit; h. Mericarp; i, Seed. [a, e-i based on Hevron 1377 (ARI), b-c based on Halse 677 (MO), d based on Wooton s.n. (US)]. 
pilose, with appressed, eglandular (and sometimes glandular) hairs; segments 5(-7), obtriangular (sometimes rhombic), 4-9 $\mathrm{mm}$ at the base, 5-7-lobed in distal half (ratio main-sinus length of the middle segment / middle-segment length $=0.13-0.19$ ); cauline leaves opposite; petioles to $19 \mathrm{~cm}$ long, with patent, eglandular hairs $0.2-1 \mathrm{~mm}$ long, and often patent, glandular hairs $0.2-0.6 \mathrm{~mm}$ long; stipules 3.5-9 × 1-2 mm, lanceolate, with eglandular (and sometimes glandular) hairs on abaxial surface and on the margin, glabrous adaxially. Inflorescence a dichasial cyme; cymules 2-flowered, solitary; peduncles 1.6-5 $\mathrm{cm}$ long, with patent, glandular hairs $0.2-0.6$ $\mathrm{mm}$ long and patent, eglandular hairs 0.1-0.5 mm long; bracteoles 3.1-4.5 × 0.7-1 mm, linear, with eglandular and glandular hairs on both sides and and on the margin; pedicels 0.9-4.1 cm long, with patent, glandular hairs $0.2-0.6 \mathrm{~mm}$ long and patent, eglandular hairs 0.1-0.5 mm long; pedicel and peduncle to- gether often overtopping the subtending leaf. Sepals 6.7-8 $\times 2.5-4 \mathrm{~mm}$ (ratio pedicel length / sepal length $=1.3-6.1$ ), smooth, not accrescent, 3-5-nerved, with mucro 0.5-1 mm long, with scarious margins $0.2-0.3 \mathrm{~mm}$ wide, with \pm patent, eglandular hairs $0.2-1(-1.4) \mathrm{mm}$ long and patent, glandular hairs $0.2-1 \mathrm{~mm}$ long on the abaxial side, glabrous adaxially. Petals 5-9(-10) $\times 1.5-5 \mathrm{~mm}$ (ratio petal length / petal width $=2-3.3$ ), patent, entire (sometimes a notch 0.5-1 mm deep), without claw, hairy on 1/2-3/4 of their adaxial surface and on the base of the abaxial one, ciliate on the basal margin, white, sometines pinkish. Stamens 10 , both whorls bearing anthers; filaments 6-8 mm long, not exserted, lanceolate, pilose on the abaxial side, ciliate on the proximal half, with hairs up tol mm long; anthers $1.2-1.8 \times 0.8-1.1 \mathrm{~mm}$. Nectaries glabrous. Gynoecium 7-8 mm long. Fruit 22-27 mm long, erect when immature; mericarps 3.8-4.5 $\times 2-2.5 \mathrm{~mm}$, without a strand of fibres,
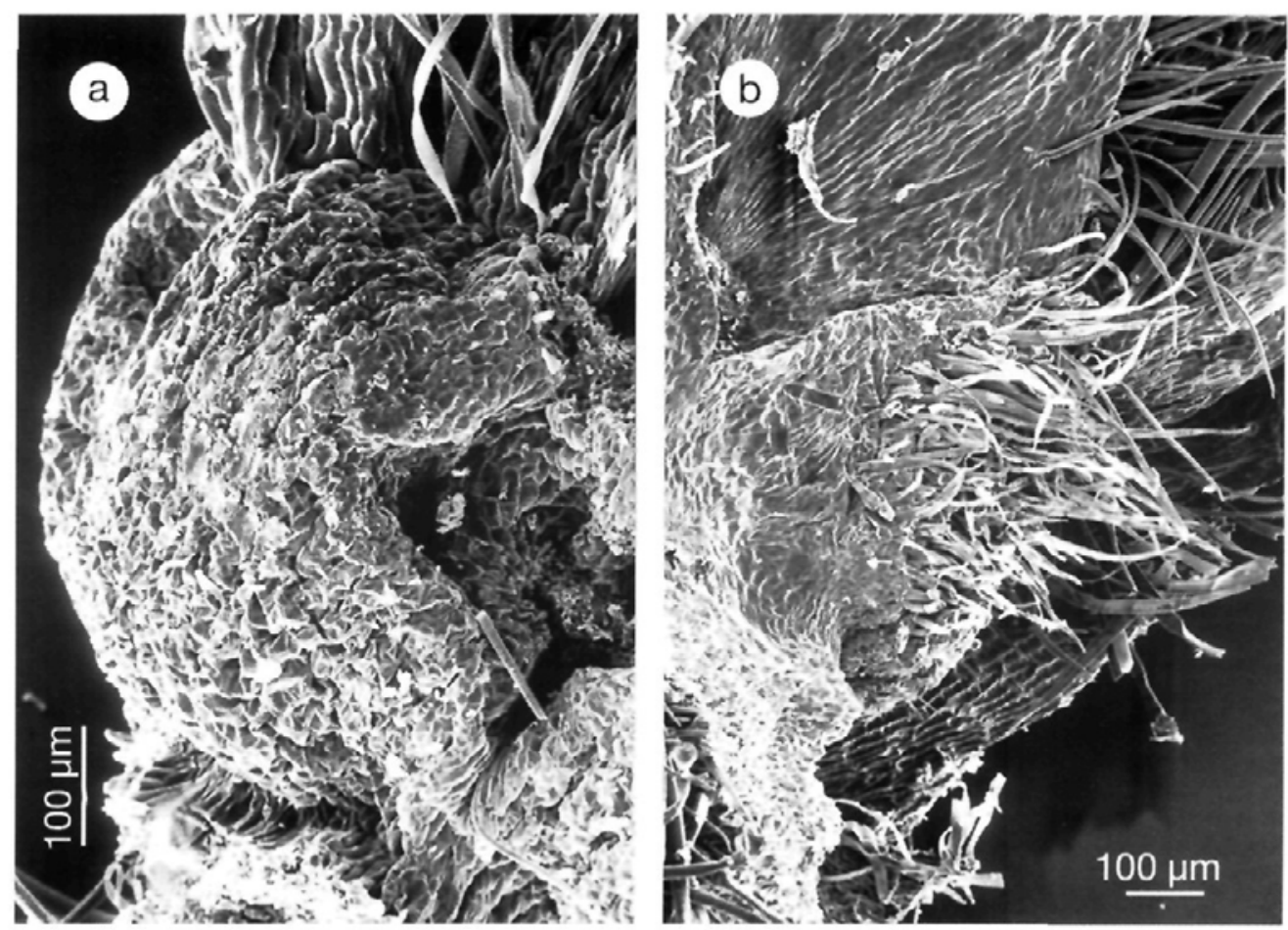

Fig. 25.-Nectaries SEM photomicrographs of: a, Geranium lentum [Lehto \& al. 20577 (NY)], and b. G. richardsonii [Kojima s.n. (DAO)]. 
smooth, with longitudinal rib, without basal beak, with a basal callus, with \pm patent, eglandular hairs $0.2-1 \mathrm{~mm}$ long, and glandular 0.2$0.7 \mathrm{~mm}$ long, brownish; rostrum $11-17 \mathrm{~mm}$ long, with a narrowed apex 1-2(-3.5) $\mathrm{mm}$ long, with \pm patent, eglandular hairs $0.1-0.2$ $\mathrm{mm}$ long, and glandular $0.3-0.6 \mathrm{~mm}$ long; stigmatic remains $4-5.2 \mathrm{~mm}$ long (ratio fruit length / stigmatic remains length $=5.2-6.5$ ), with 5 glabrous lobes. Seeds $2.5-2.9 \times 1.7$ $\mathrm{mm}$, reticulate, brownish; hilum $1 / 5$ as long as the perimeter. Cotyledons entire.

Flowering June-September. Coniferous forests (also in oak or aspen woods), roadsides, or lava beds; $2100-2600 \mathrm{~m}$. Southwestern U.S.A. \& South-Central U.S.A. Fig. 26.

Illustrations. Fig. 8c, 24, 25a.

Geranium lentum is characterised by the glabrous nectaries, and the stigmatic remains 4-5 mm long. Geranium wislizenii is morphologically alike to $G$. lentum, with which it shares short petals and sepals. However, the former has nectaries dorsally hairy, and stig- matic remains $1.5-3 \mathrm{~mm}$ long. I have not found fully eglandular specimen of $G$. lentum, although some lack glandular hairs on the stem.

JONES \& JONES (1943: 51) recorded $G$. lentum from Texas [Chisos Mts, Mueller 8050 (F, TEX)], more than $500 \mathrm{~km}$ southeast of the nearest locality of this species. However, I regard this collection as G. caespitosum. Other mentions seem to be based on the same specimens (CORREL \& JOHNSTON 1979: 891; http://www.csdl.tamu.edu/FLORA/res/plants .html). Geranium lentum would also grow in Mexico, although I have seen no specimens of it.

\section{Representative specimens examined}

USA. ARIZona: Apache Co., $20 \mathrm{mi}$ S of Saint Johns, $34^{\circ} 30^{\prime} \mathrm{N}, 109^{\circ} 21^{\prime} \mathrm{W}, 26-\mathrm{VIII}-1973$, Lehto \& al. 11564 (NY ARIZ); Apache Co.. Chusca Mountains, Sonsela Buttes. $36^{\circ} 6^{\prime} \mathrm{N}, 109^{\circ} 6$ 'W, 20 VIII-1991, Hevron 1377 (ARIZ); Apache Co., Upper Canyon del Muerto, Canyon de Chelly National Monument, $36^{\circ} 8^{\prime} \mathrm{N}, 109^{\circ} 29^{\prime} \mathrm{W}, 12$-VII1971, Halse 483 (ARIZ MO); Apache Co.,

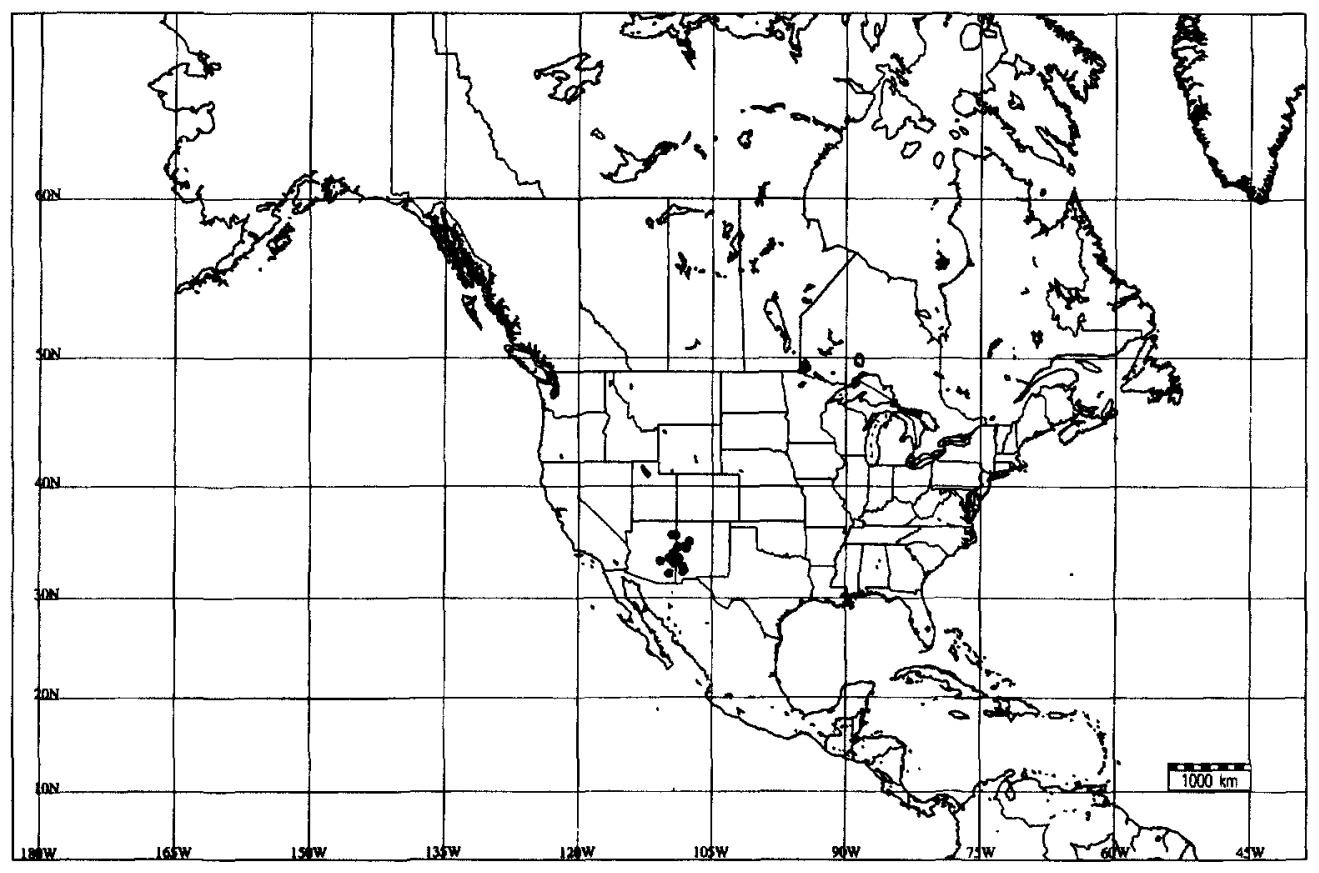

Fig. 26.--Distribution of Geranium lentum. 
Williams Creek Youth Camp, 34 $3^{\circ} \mathrm{N}, 109^{\circ} 49^{\prime} \mathrm{W}$, 13-VIII-1970, Grafelt 70-120 (ARIZ); Graham Co., Coronado National Forest, Pinaleno Mountains, at Wet Canyon Campground, $32^{\circ} 39^{\prime} \mathrm{N}$, $109^{\circ} 48^{\prime} \mathrm{W}, 11-\mathrm{IX}-1976, \mathrm{Mc}$ Gill \& Lehto 20577 (NY); Greenlee Co., $10 \mathrm{mi} S$ of Alpine on US route $666,33^{\circ} 35^{\prime} \mathrm{N}, 109^{\circ} 15^{\prime} \mathrm{W}, 28-\mathrm{VI}-1992$, Miller \& al. 7668 (MO); Greenlee Co., Beaver Creek, between Hannagan Meadows and Buffalo Crosing, $33^{\circ} 43^{\prime} \mathrm{N}, 109^{\circ} 21^{\prime} \mathrm{W}, 23-\mathrm{VIII}-1969$, White s.n. (ARIZ MO, UC); Greenlee Co., Beaver Creek, near Sprucedale, White Mountains, $33^{\circ} 43^{\prime} \mathrm{N}, 109^{\circ} 21^{\prime} \mathrm{W}, 30$-VIII-1951, Parker \& McClintock 7644 (NY ARIZ. MO, UC). NEW MEXICo: Catron Co., Sapillo Creek, 35 $34^{\prime N}$, $107^{\circ} 31^{\prime}$ W, 31-VII-1981, Knight 1681 (UNM); Catron Co., West Fork of Gila, 33 $46^{\prime} \mathrm{N}$, $110^{\circ} 49^{\prime} \mathrm{W}, 7-\mathrm{VIII}-1900$, Wooton s.n. (US); Cibola Co., Zuñi Mountains, Malpais Spring. 35 $5^{\prime} \mathrm{N}$, $108^{\circ} 1^{\prime} \mathrm{W}, 11-\mathrm{VII}-1985$, Fletcher 8327 (UNM); Grant Co., Pinos Altos Mountains, $32^{\circ} 51^{\prime} \mathrm{N}$, $108^{\circ} 14^{\prime} \mathrm{W},-\mathrm{IX}-1880$, Greene s.n. (F); Grant Co., Redstone, $32^{\circ} 56^{\prime} \mathrm{N}, 108^{\circ} 11^{\prime} \mathrm{W}, 13-\mathrm{VIII}-1893$, Mulford 834 (MO); Johnson Basin, $34^{\circ} 4^{\circ} \mathrm{N}$, $108^{\circ} 46^{\prime} \mathrm{W}, 10-\mathrm{VII}-1978$, E.O.W. 163 (NMC); Mckinley Co., Rio Zuñi, 35 $4^{\prime}$ N, $108^{\circ} 50^{\prime} \mathrm{W}, 27$ VII-1892, Wooton 353 (NMC); Mogollon Mountains, Indian Creek, $33^{\circ} 18^{\prime} \mathrm{N}, 108^{\circ} 19^{\prime} \mathrm{W}, 10$-VII1978, Moir \& Fitzhugh 586 (NMC); Valencia Co., Bonita Canyon, $34^{\circ} 57^{\prime} \mathrm{N}, 107^{\circ} 56^{\prime} \mathrm{W}, 14-\mathrm{VI}-1969$. Riffle 1113 (UNM).

15. Geranium wislizenii $S$. Watson in Proc. Amer. Acad. Arts 21: 421 (1886) ["Wislizeni"]

Ind. loc.: "Norogachi, in clefts of rocks (428) [collected by E. Palmer]; also collected by Dr. Wislizenus on the Llanos mountains, Chihuahua, and referred by Dr. Engelmann (Pl. Fendler, 27) to G. Hernandezii, and by myself (Proc. Amer. Acad. 17. 334) to G. Mexicanum"

Typus: Mexico. Chihuahua, $150 \mathrm{mi} \mathrm{N}$ of Batopilas, Nov. 1885, Palmer 428 [lectotype designated by Moore (1943: 33), $\mathrm{GH}$ !; isolectotypes, LE! $\mathrm{K}$ ! US] [26 $26^{\prime} \mathrm{N}$, $\left.106^{\circ} 48^{\prime} \mathrm{W}\right]$

$=G$. geoides Small in Underw. \& Britton (eds.), N. Amer. Fl. 25(1): 19 (1907)

Ind. loc.: "Type collected in the Sierra Madre, Chihuahua, Mexico, September 17, 1887, C. G. Pringle 1203"
Typus: Mexico. Chihuahua, Sierra Madre, 17 Sep. 1887, Pringle 1203 (holotype, NY; isotypes, F! GH MEXU! US)

$=G$. calvescens Briq. in Annuaire Conserv. Jard. Bot. Genève 11-12: 186 (1908)

Ind. loc.: "Mexicum: in civitate Chihuahua, in faucibus Sierra Madre, 14 oct. f. fr. 1888 (Pringle n. 1578)"

Typus: Mexico. Chihuahua, in faucibus Sierra Madre, 14 Oct. 1888, Pringle 1578 [lectotype, designated by KNUTH (1912: 201), G; isolectotypes, BM! MO P! UC! W!]

$=$ G. durangensis Rose, nom. nud., in sched.

Herbs perennial, 20-60 cm tall. Rootstock 3-9 mm diam., vertical, not tuberculate, not turnip-shaped. Stem erect, usually few per caudex branch, with patent to retrorse, appressed, eglandular hairs 0.3-1.2 mm long. Basal leaves in a persistent rosette; lamina $3.2-5.9 \times 3.6-7.1 \mathrm{~cm}$, polygonal in outline, cordate, palmatifid (divided for 0.59-0.75 of its length), pilose, with appressed, eglandular (and sometimes glandular) hairs; segments 5, rhombic, 6-12 $\mathrm{mm}$ at the base, 5-13-lobed in distal half (ratio main-sinus length of the middle segment $/$ middle-segment length $=0.13$ 0.20 ); cauline leaves opposite; petioles to 20 $\mathrm{cm}$ long, with patent to retrorse, eglandular hairs 0.3-1.5 mm long; stipules 5-18 x 1-2 $\mathrm{mm}$, lanceolate, with eglandular hairs on both sides and on the margin. Inflorescence a dichasial cyme; cymules 2-flowered, solitary; peduncles $2-7.2 \mathrm{~cm}$ long, with patent, glandular hairs $0.2-0.7 \mathrm{~mm}$ long and patent to retrorse, eglandular hairs 0.2-1.2 mm long, sometimes lacking glandular hairs; bracteoles 3-5 $\times$ 0.5-0.7 mm, linear, with eglandular hairs on abaxial surface and on the margin, glabrous adaxially; pedicels $1.8-2.3 \mathrm{~cm}$ long, with patent, glandular hairs $0.2-0.7 \mathrm{~mm}$ long and patent to retrorse, eglandular hairs $0.2-1.2$ $\mathrm{mm}$ long, sometimes lacking glandular hairs; pedicel and peduncle together often overtopping the subtending leaf. Sepals 4.5-6(-7) $\times$ 1.9-2.4 mm (ratio pedicel length / sepal length = 3.1-4.5), smooth, not accrescent, 3-5nerved, with mucro $0.7-1.2 \mathrm{~mm}$ long, with scarious margins $0.1-0.2 \mathrm{~mm}$ wide, with 


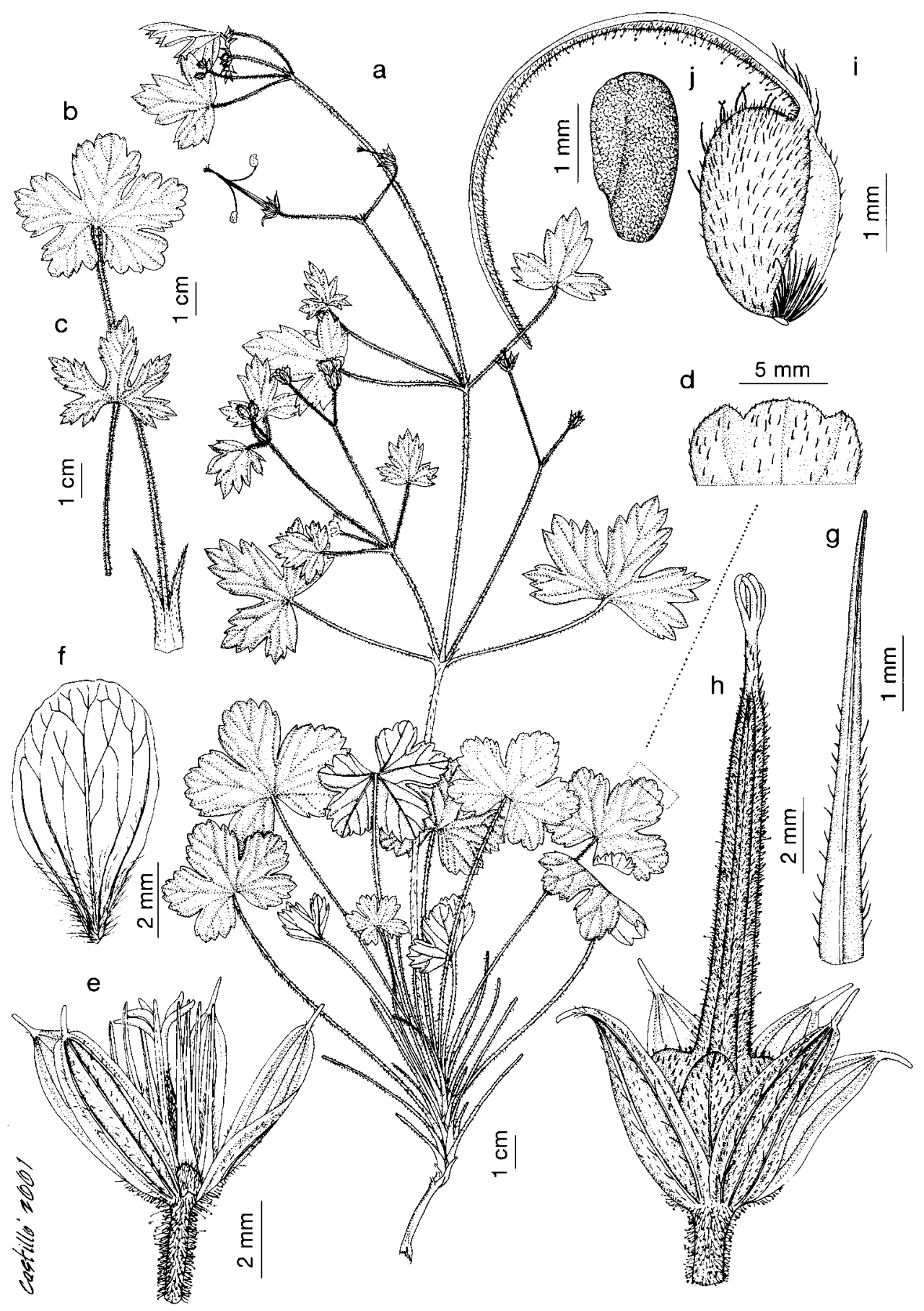

Fig. 27.-Geranium wislizenii: a, Habit; b, Basal leaf; $c$, Caulinar leaf; d, Detail of leaf adaxial surface; e, Flower without petals; f, Petal; g, Staminal filament; h, Fruit; i, Mericarp; j, Seed. [a, d-j based on Sueur 733 (TEX), b based on Shreve 5029 (UC), e based on Spellenberg \& al. 12230 (NMC)]. 
antrorse to patent, eglandular hairs $0.3-1.1$ $\mathrm{mm}$ long, and occasionally glandular, patent hairs $0.2-0.6 \mathrm{~mm}$ long on the abaxial side, glabrous adaxially. Petals 6-9(-10.5) × 3.5-6 $\mathrm{mm}$ (ratio petal length / petal width $=1.5-2.3$ ), erect-patent, entire, without claw, hairy on 1/3-1/2 of their adaxial surface and on the base of the abaxial one, ciliate on the basal margin, white. Stamens 10, both whorls bearing anthers; filaments 4-6 mm long, not exserted, lanceolate, pilose on the abaxial side, ciliate on the proximal half, with hairs up to $0.5 \mathrm{~mm}$ long; anthers $1-1.1 \times 0.5-0.9 \mathrm{~mm}$. Nectaries dorsally hairy. Gynoecium $4-5 \mathrm{~mm}$ long. Fruit 17-21 mm long, erect when immature; mericarps $2.4-3.5 \times 1.5-1.9 \mathrm{~mm}$, without a strand of fibres, smooth, with longitudinal rib, without basal beak, with a basal callus, with \pm patent, eglandular hairs $0.1-0.6 \mathrm{~mm}$ long, and, sometimes, glandular hairs 0.2-0.4 mm long, brownish; rostrum 11.3-15.2 mm long, with a narrowed apex 1.6-2.5 mm long, with \pm patent, eglandular hairs $0.1-0.3 \mathrm{~mm}$ long, and glandular hairs 0.4-0.6 mm long, sometimes lacking glandular ones; stigmatic remains $1.5-3 \mathrm{~mm}$ long (ratio fruit length / stigmatic remains length $=5.6-9.5$ ), with 5 glabrous lobes. Seeds $2.3 \times 1.3 \mathrm{~mm}$, reticulate, brownish; hilum $1 / 4$ as long as the perimeter. Cotyledons entire.

Flowering August-September. Coniferous forests (also in oak woods) or rocky slopes; 200-3300 m. Southwestern U.S.A., SouthCentral U.S.A. \& North Mexico. Fig. 28.

Illustrations. Fig. 5d, 8d, 27.

This species varies in distribution of glandular indumentum in a similar way to $G$. caespitosum. Thus, it cannot be recognised on the basis of the lack of glandular hairs as proposed by JONES \& JONES (1943). Differences and similarities between $G$. wislizenii and $G$. lentum are addressed in the discussion of the latter species. Geranium wislizenii would also be related to G. richardsonii, since they share nectaries dorsally hairy and white petals. However, $G$. richardsonii has longer petals and sepals.

JONES \& JONES (1943: 52) recorded G. wislizenii from Texas [Mt. Livermore, Hinckley
404 (F)]. Unfortunately this specimen is not well enough developed to be unequivocally identified. No other specimens of $G$. wislizenii from Texas have been located.

\section{Representative specimens examined}

Mexico. Mexico Northeast: Chihuahua, $150 \mathrm{mi} \mathrm{N}$ of Batopilas, -XI-1885, Palmer 428(GH); $4.7 \mathrm{mi}$ E Mesa del Huracan on road to Las Varas, $29^{\circ} 39^{\prime} \mathrm{N}, 108^{\circ} 14^{\prime} \mathrm{W}$, 21-VIII-1980, Warnock \& McCormick 2120 (TEX); 46 mi W of Parral on road to Vergel, 6-X-1959, Correll \& Gentry 22866 (TEX); Arroyo El Cebollín between El Oso and Sierra Oscura, 28 $10^{\prime}$ N, 108 ${ }^{\circ} 9^{\prime} \mathrm{W}, 9-X I-1986$, Howell s.n. (ARIZ); at Tonichic on Arroyo Chorro de Agua, $28^{\circ} 13^{\prime} \mathrm{N}, 108^{\circ} 6^{\prime} \mathrm{W}, 30$-IX-1986, Martin \& $O^{\prime}$ Rourke 129 (TEX); Basaseachic, 28 ${ }^{\circ} 14^{\prime} \mathrm{N}, 108^{\circ} 12^{\prime} \mathrm{W}$, 15-X-1985, Eastoe s.n. (NMC); Bermudez, $28^{\circ} 15^{\prime} \mathrm{N}$, $109^{\circ} 2^{\prime} \mathrm{W}, 18$-VIII-1985, Turner 85-89 (ARIZ); Bocoyna, $27^{\circ} 50^{\prime} \mathrm{N}, 107^{\circ} 35^{\prime} \mathrm{W}, 31$-VIII-1978, Bye 9084 (MEXU, UC); cascada de Basaseachic, $28^{\circ} 10^{\prime} \mathrm{N}$, $108^{\circ} 13^{\prime} \mathrm{W}, 23-\mathrm{VII}-1986$, Martin \& al. s.n. (NMC); Chuhuichupa, 29'38'N, $108^{\circ} 22^{\prime}$ W, -VIII-1936, LeSueur 733 (F TEX); Cusarare, NE cascada de Cusarare, $27^{\circ} 37^{\prime} \mathrm{N}, 107^{\circ} 32^{\prime} \mathrm{W}, 20$-VIII-1978, Bye 8719 (MEXU, UC); El Rayo, $27^{\circ} 15^{\prime} \mathrm{N}, 108^{\circ} 37^{\prime} \mathrm{W}, 14-\mathrm{X}-1988$, Jenkins \& al. 88-130 (ARIZ); Gómez Farias, laguna de Babícora, $29^{\circ} 15^{\prime} \mathrm{N}, 107^{\circ} 45^{\prime} \mathrm{W}, 18-\mathrm{VIII}-1994$, Quintana \& $E$. Estrada 3099 (NMC, TEX); La Mesa Colorado. $25^{\circ} 52^{\prime} \mathrm{N}, 106^{\circ} 39^{\prime} \mathrm{W}, 14-\mathrm{X}-1933$, Gentry 544 (ARIZ); Madera, camino Nicolas Bravo-Las Varas, $29^{\circ} 39^{\prime} \mathrm{N}$, 107²2'W, 18-VIII-1994, Quintana \& Estrada 3006 (NMC); Majalca, 28 ${ }^{\circ} 13^{\prime} \mathrm{N}, 1^{\circ} 6^{\circ} 34^{\prime} \mathrm{W}, 26-\mathrm{IX}-1997$, Yen \& Estrada 8731 (NMC); Nabogame, $12 \mathrm{~km} \mathrm{NW}$ of Yepachic, $28^{\circ} 30^{\prime} \mathrm{N}, 108^{\circ} 30^{\prime} \mathrm{W}, 24-\mathrm{X}-1986$, Laferriere 328 (ARIZ, MEXU, SD); Ocampo, P.N. Cascada de Basaseachi, Rancho de San Lorenzo, $28^{\circ} 10^{\prime} \mathrm{N}$, $108^{\circ} 13^{\prime} \mathrm{W}, 25-\mathrm{IX}-1994$, Spellenberg \& al. 12230 (MEXU, NMC); Pichachic, $28^{\circ} 8^{\prime} \mathrm{N}, 107^{\circ} 28^{\prime} \mathrm{W}, 25-\mathrm{VI}-$ 1937, Shreve 8017 (ARIZ); Río Fuertes, Sierra Charuco, 11-IX-1935, Gentry 1756 (ARIZ, F); Río Mayo, Cerro Quicorichi, 6-X-1935, Gentry 1937 (ARIZ); Río Mayo, Los Cascarones, 19-IX-1936, Gentry 2804 (ARIZ, F); road to Basaseachic through Tomochic, $28^{\circ} 20^{\prime} \mathrm{N}$, $107^{\circ} 51^{\prime} \mathrm{W}, 26-$ VIII-1979, Lane 2750 (TEX); Sierra Charuco, rancho Byerly, -1946, Langille 220 (ARIZ); Sierra Madre near Colonia García, $29^{\circ} 59^{\prime} \mathrm{N}, 108^{\circ} 20^{\prime} \mathrm{W}$, 5-VIII-1899, Rose 222 (F, NMC, P, UC); Sierra Mohinora, $26^{\circ} 6^{\prime} \mathrm{N}, 107^{\circ} 4^{\prime} \mathrm{W}, 16-\mathrm{X}-1959$, Correll \& Gentry 23159 (TEX); SW of Buenaventura, Rancho de la Tinaja, $29^{\circ} 42^{\prime} \mathrm{N}, 107^{\circ} 35^{\prime} \mathrm{W}, 30$-VIII-1989, Mayfield 179 (TEX); Temosachic, SE of Madera, Cañon Huahuatán, $29^{\circ} 4^{\prime} \mathrm{N}, 107^{\circ} 51^{\prime} \mathrm{W}, 24-\mathrm{IX}-1939$, Muller 3457 (UC); Vieja Casas Grandes, 'N, 'W, 26-VIII-1952, Tucker 2534 (ARIZ, UC); DURANGo, canyon of the Río Chico, $18 \mathrm{mi}$ W of Durango, $24^{\circ} 2^{\prime} \mathrm{N}, 104^{\circ} 50^{\prime} \mathrm{W}, 24-\mathrm{VII}-1958$, Correl \& I.M. Johnston 20090 (P, TEX, UC); Sandia Station, $26^{\circ} 36^{\prime} \mathrm{N}, 106^{\circ} 4^{\prime} \mathrm{W}, 19-\mathrm{X}-1905$, Pringle 10022 (F, MEXU, P, UC): SONORA, Cerro de Capulín, NW of Aribabi, $29^{\circ} 18^{\prime} \mathrm{N}, 109^{\circ} 18^{\prime} \mathrm{W}, 4-\mathrm{IX}-1939$, White 2719 (ARIZ); El Rancho de la Nacha, $30^{\circ} 28^{\prime} \mathrm{N}, 109^{\circ} 40^{\prime} \mathrm{W}, 14$ - 


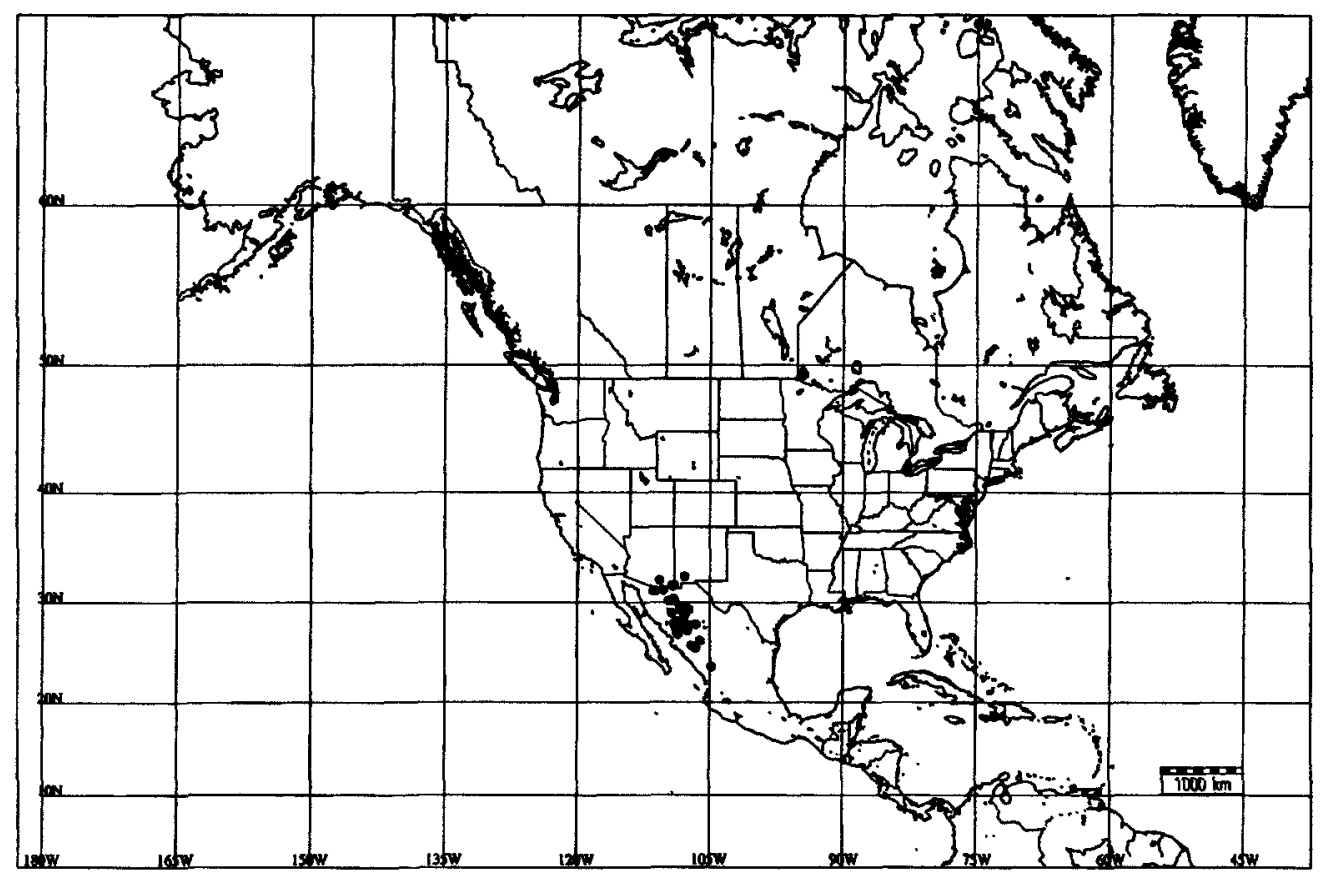

Fig. 28.-Distribution of Geranium wislizenii.

VIII-1941, White 3978 (TEX); El rancho del Roble, NE of El Tigre, $30^{\circ} 40^{\prime} \mathrm{N}, 109^{\circ} 5^{\prime} \mathrm{W}, 2-\mathrm{IX}-1941$, White 4198 (MEXU, TEX); El Tigre Canyon, East of Esqueda and lago Angostura, $30^{\circ} 34^{\prime} \mathrm{N}, 109^{\circ} 11^{\prime} \mathrm{W}, 9-\mathrm{IX}-1961$, Turner \& al. 2106 (ARIZ); Mesa El Otro Lado, $1 \mathrm{~km} \mathrm{NE}$ of Yecora, $28^{\circ} 23^{\prime} \mathrm{N}, 108^{\circ} 54^{\prime} \mathrm{W}, 7-1 \mathrm{X}-1995$, Devender 95-826 (ARIZ); puerto de los Aserraderos, $30^{\circ} 28^{\prime} \mathrm{N}, 109^{\circ} 1$ 'W, 9-VIII-1940, White 3147 (ARIZ); Río Maycoba, $28^{\circ} 23^{\prime} \mathrm{N}, 108^{\circ} 46^{\prime} \mathrm{W}, 20-\mathrm{X}-1991$, Joyal \& Enriques 1858 (TEX); Río Mayo, Saguaribo, $27^{\circ} 21^{\prime} \mathrm{N}, 108^{\circ} 40^{\prime} \mathrm{W}, 2-$ XI-1935, Gentry 2115 (F, UC).

USA. ARIzona: Cochise Co., Chiricahua Mountains, Cave Creek, 31 ${ }^{\circ} 58^{\prime} \mathrm{N}, 109^{\circ} 3^{\prime} \mathrm{W}, 20$-VIII-1981, Darrow \& al. 1301 (ARIZ); Cochise Co., Chiricahua Mountains, Cave Creek Canyon, $31^{\circ} 58^{\prime} \mathrm{N}, 109^{\circ} 3^{\prime}$ 'W. 20-VIII-1981, Fishbein \& Carey 2113 (ARIZ); Cochise Co., Chiricahua Mountains, S of Portal, Southwest Field Station, $31^{\circ} 54^{\prime} \mathrm{N}, 109^{\circ} 8^{\prime} \mathrm{W}, 21-\mathrm{VIII}-1959$, Norland \& al. 130 (SD); Cochise Co., Chiricahua Mountains, South Fork of Cave Creek, 31 ${ }^{\circ} 58^{\prime} \mathrm{N}, 109^{\circ} 3^{\prime} \mathrm{W}, 24-\mathrm{IX}-1961$, Bequaert s.n. (ARIZ); Cochise Co., Huachuca Mountains, Garden Canyon, $31^{\circ} 29^{\prime} \mathrm{N}, 110^{\circ} 19^{\prime} \mathrm{W}, 5-\mathrm{IX}-1959$, Goodding 233-59 (ARIZ); Cochise Co., Huachuca Mountains, Ramsey Canyon, $31^{\circ} 27^{\prime} \mathrm{N}, 10^{\circ} 17^{\prime} \mathrm{W}, 22-\mathrm{VIII}-1910$, Goodding 729 (ARIZ NY); Cochise Co., South Fork 5 $\mathrm{mi}$ SW of Portal, $31^{\circ} 52^{\prime} \mathrm{N}, 109^{\circ} 11^{\prime} \mathrm{W}, 20$-VIII-1981, Buchanan \& Hodgson 1508 (SD); Pima Co., Santa Catalina Mountains, $32^{\circ} 25^{\prime} \mathrm{N}, 110^{\circ} 42^{\prime} \mathrm{W}, 5-\mathrm{IX}-1904$, Griffiths 7073 (MO); Santa Cruz Co., Sycamore Canyon, ca. $1 / 4 \mathrm{mi}$ below Yank Spring, $31^{\circ} 25^{\prime} \mathrm{N}$, $111^{\circ} 11^{\prime} \mathrm{W}, 17-\mathrm{IX}-1951$, Kaiser 498 (ARIZ); Santa Cruz Co., Sycamore Canyon, near Ruby, $31^{\circ} 25^{\prime} \mathrm{N}$, $111^{\circ} 23^{\prime} \mathrm{W}, 30-\mathrm{IX}-1944$, Darrow \& Haskell 2040 (ARIZ, F, NY). NEw MexICo: Grant Co., Black Range, Gallinas Creek, $32^{\circ} 44^{\prime} \mathrm{N}, 107^{\circ} 51^{\prime} \mathrm{W}, 24-\mathrm{VIII}-1969$, Todsen s.n. (NMC).

16. Geranium richardsonii Fisch. \& Trautv. in Fisch., C.A. Mey. \& Trautv., Index Sem. Hort. Petrop. 4: 37 (1838)

G. albiflorum Hook., Fl. Bor.-Amer. 1: 116 , tab. 40 (1831) [syn. subst.], nom. illeg., non Ledeb. (1829)

G. hookerianum Walp., Repert. Bot. Syst. 1: 450 (1842), nom. illeg.

Ind. loc.: "Hab. Vallies in the Rocky Mountains. Drummond"

Typus: Canada. Rocky Mountains, Drummond s.n. (lectotype, here designated, $\mathbf{K}$ !)

$=$ G. gracilentum Greene in Rydb. in Agric. Exp. Sta. Agric. Coll. Colorado Bull. 100: 218 (1906)

Ind. loc.: "In mountain valleys from Colo. to N.M. and Ariz.-Alt. 6000-10,000 ft.- 
Headwaters of Clear Creek; Pike's Peak; Piedra; Mancos; 4 milles west of Cameron Pass; near Veta Pass; near La Plata Post Office; La Plata Cañon; Columbine, Middle Park; Box Cañon, west of Ouray; Bosworth's; Beaver Creek; bank of Elk River, Routt Co.; Trail Creek bottom; Rico; Silverton; northwest of Dolore"

Typus: USA. Colorado, Archuleta Co., Piedra, Jul. 1899, Baker 449 (lectotype, here designated, $\mathrm{MO}$ !; isolectotypes, $\mathrm{BM}$ ! F! GH! NY P! W! COI!) [see also JONES \& JONES (1943: 35)]

$=$ G. loloense H. St. John, Fl. Southeast Wash. \& Idaho: 242 (1937)

Ind. loc.: "Idaho: Musselshell, Lolo Trail, Bitterroot Mts., (T. 35 N., R. 6 E., Clearwater Co.), July 14, 1902, C.V. Piper 4027 (type in Herb. State College of Washington)"

Typus: USA: Idaho, Clearwater Co., Musselshell, Lolo Trail, Bitterroot Mts., 14 July 1902, Piper 4027 (no authentic material located)

Herbs perennial, $15-90 \mathrm{~cm}$ tall. Rootstock 7-12 mm diam., horizontal, not tuberculate, not turnip-shaped. Stem erect, solitary, with patent to retrorse, eglandular hairs $0.3-0.6$ $\mathrm{mm}$ long, and sometimes patent, glandular hairs 0.3-0.9 mm long. Basal leaves in a persistent rosette; lamina (3.9-)4.5-9.1 × (4.4-)6$11.3 \mathrm{~cm}$, polygonal in outline, cordate, palmatifid (divided for 0.75-0.89 of its length), pilose, with appressed, eglandular hairs; segments 5-7, rhombic, 5-12 $\mathrm{mm}$ at the base, 6-12-lobed in distal half (ratio main-sinus length of the middle segment / middlesegment length $=0.17-0.25$ ); cauline leaves opposite; petioles to $36 \mathrm{~cm}$ long, with patent to retrorse, eglandular hairs $0.5-0.9 \mathrm{~mm}$ long, and sometimes patent, glandular hairs 0.3-0.9 mm long; stipules 9-11 $\times 3-4 \mathrm{~mm}$. lanceolate, with eglandular hairs on abaxial surface and on the margin, usually glabrous adaxially. Inflorescence a dichasial cyme; cymules 2flowered, solitary; peduncles $2.5-8.7 \mathrm{~cm}$ long, with patent, glandular hairs $0.2-0.9 \mathrm{~mm}$ long and patent to retrorse, eglandular hairs 0.1-0.4 mm long, rarely lacking glandular hairs; bracteoles 4-10 × 1-1.5 mm, linear-lanceo- late, with eglandular hairs on abaxial surface and on the margin, glabrous adaxially; pedicels $1.5-3 \mathrm{~cm}$ long, with patent, glandular hairs $0.2-0.9 \mathrm{~mm}$ long and patent to retrorse, eglandular hairs 0.1-0.4 mm long, rarely lacking glandular hairs; pedicel and peduncle together often overtopping the subtending leaf. Sepals 7-8 $\times 3-4 \mathrm{~mm}$ (ratio pedicel length / sepal length $=2.4-4$ ), smooth, not accrescent, 3-5-nerved, with mucro 1-3 mm long, with scarious margins $0.1-0.2 \mathrm{~mm}$ wide, with antrorse, appressed, eglandular hairs 0.2-0.4 $\mathrm{mm}$ long and patent, glandular hairs 0.3-0.9 $\mathrm{mm}$ long on the abaxial side, glabrous adaxially. Petals $14-20 \times 8-15 \mathrm{~mm}$ (ratio petal length $/$ petal width $=1.3-1.7$ ), erect-patent, entire, without claw, hairy on 1/3-1/2 of their adaxial surface, ciliate on the basal margin, white. Stamens 10, both whorls bearing anthers; filaments $8-9 \mathrm{~mm}$ long, not exserted, lanceolate, pilose on the abaxial side, ciliate on the proximal half, with hairs up tol $\mathrm{mm}$ long; anthers $1.7 \times 0.8 \mathrm{~mm}$. Nectaries dorsally lanate. Gynoecium 8-9 mm long. Fruit 19 $26 \mathrm{~mm}$ long, erect when immature; mericarps $3.5-4 \times 2-2.5 \mathrm{~mm}$, without a strand of fibres, smooth, with longitudinal rib, without basal beak, with a basal callus, with \pm patent, eglandular hairs 0.1-0.3 mm long, and glandular 0.5-0.9 mm long, brownish; rostrum 13-17 $\mathrm{mm}$ long, with a narrowed apex 1-2 mm long, with \pm patent, eglandular hairs $0.1-0.3 \mathrm{~mm}$ long, and glandular $0.4-0.8 \mathrm{~mm}$ long; stigmatic remains 3-4 mm long (ratio fruit length / stigmatic remains length $=5.5-8.3$ ), with 5 glabrous lobes. Seeds $3-3.2 \times 1.5-1.7 \mathrm{~mm}$, reticulate, brownish; hilum 1/4 as long as the perimeter. Cotyledons entire. $n=26,28$.

Flowering May-September. Coniferous forests (also in aspen or willow woods), forested roadsides or moist meadows; 6003800 m. Subartic America, Western Canada, Northwestern U.S.A., Southwestern U.S.A., North-Central U.S.A. \& South-Central U.S.A. Fig. 29.

Illustrations. Fig. 25b, 29, $30 \mathrm{~b}$.

Geranium richardsonii is readily recognised by its white long petals, hairy on the 


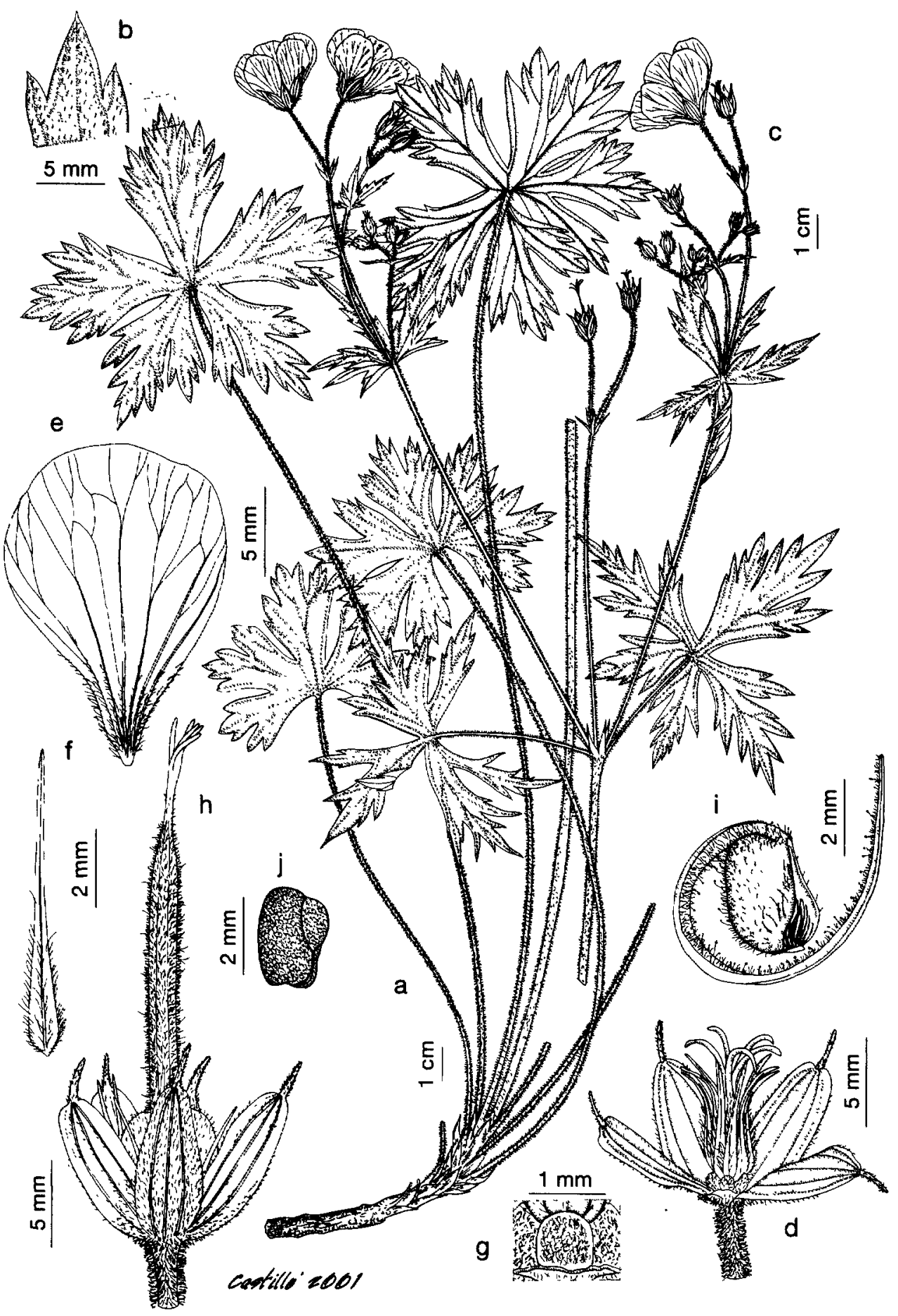

Fig. 29.-Geranium richardsonii: a, Habit; b, Detail of leaf adaxial surface; c, Inflorescence; d, Flower without petals. e, Petal; f, Staminal filament; g, Nectary; h, Fruit; i, Mericarp; j, Seed. [a-g based on Siplivinsky 1755 (MA), h-j based on Aedo 4435 (MA)]. 
adaxial surface, and its hairy nectaries. In some specimens from Utah, Colorado and California nectaries indumentum is scarce and difficult to see. The presence of glandular hairs varies considerably (as in $G$. caespitosum). There are specimens with abundant glandular hairs on the stem base, petioles and inflorescence, while others only have scarce glandular hairs on pedicels. Surprisingly, this variability has not originated the description of a high number of taxa, as in $G$. caespitosum.

This species could be related to $G$. albidum Rydb. ex Hanks \& Small, a Mexican species also with hairy nectaries. However, G. albidum differs by its smaller petals, and eglandular sepals.

The specimens supporting the records of G. richardsonii from Washington (KARTESZ 1998) have not been studied.

\section{Representative specimens examined}

Canada. Alberta: $10 \mathrm{mi}$ E of Ponoka, 52 $42^{\prime} \mathrm{N}$, $113^{\circ} 35^{\prime} \mathrm{W}, 12-\mathrm{VII}-1949$, Frankton 914 (DAO); $3 \mathrm{mi} \mathrm{SW}$ of Pincher Creek, $49^{\circ} 29^{\prime} \mathrm{N}, 113^{\circ} 57^{\prime} \mathrm{W}, 9$-VIII-1950, Dore \& Breitung I2444 (DAO); Bow valley, pr. Calgary, $51^{\circ} 3^{\prime} \mathrm{N}, 1_{11}^{\circ} 5^{\prime} \mathrm{W}, 26-\mathrm{VII}-1913$, Moodie 45 (F); Bowden, $51^{\circ} 55^{\prime} \mathrm{N}, 114^{\circ} 2^{\prime} \mathrm{W}$, Willing 334 (DAO); Buck Lake. $52^{\circ} 33^{\prime} \mathrm{N}, 117^{\circ} 39^{\prime} \mathrm{W}, 28-\mathrm{VI}-1968$, Dumais \& Anderson 2789 (DAO); Elkton, 51 $39^{\circ} \mathrm{N}, 114^{\circ} 32^{\prime} \mathrm{W}$, -VIII-, Dore s.n. (DAO); For Saskatchewan, $53^{\circ} 43^{\prime} \mathrm{N}, 113^{\circ} 13^{\prime} \mathrm{W}, 2-$ VIIII-1940, Turner 2145 (DAO); Handhills, $51^{\circ} 30^{\prime} \mathrm{N}$, $112^{\circ} 17^{\circ} \mathrm{W}, 11-\mathrm{VII}-1943$, Brinkman 5462 (DAO); Kananaskis Prov. Park, mt. Elpoca, $51^{\circ} 4^{\prime} \mathrm{N}, 115^{\circ} 8^{\prime} \mathrm{W}$, 28-VI-1977, Brauton 1386 (DAO): Lacombe, $52^{\circ} 28^{\prime} \mathrm{N}$, $113^{\circ} 44^{\prime}$ W. 18-VII-1926, Dixon 684 (DAO); Lesser Slave Lake, $55^{\circ} 27^{\prime} \mathrm{N}, 115^{\circ} 27^{\prime} \mathrm{W}, 14$-VII-1929, Brinkman 4390 (DAO): Mt. Robson Provincial Park, trail to Kinney Lake. $53^{\circ} 10^{\prime} \mathrm{N}, 119^{\circ} 8^{\prime} \mathrm{W}, 29-\mathrm{VII}-1950, \mathrm{Ha}$ gen s.n. (WTU); N of Coleman. $49^{\circ} 39^{\prime} \mathrm{N}, 114^{\circ} 30^{\prime} \mathrm{W}, 15-$ VIII-1955, Kruk \& McElrov 111 (DAO): Starkey's Ranch. Kananaskis Forest Expt., $50^{\circ} 50^{\circ} \mathrm{N}, 115^{\circ} 15^{\circ} \mathrm{W}$. 14-VII-1950. Whitney 50/222 (DAO): Waterton National Park, 49 ${ }^{\circ}$ 'N, $116^{\circ} 52^{\prime} \mathrm{W}, 27$-VIII-1980, Parmelee 5397 (DAO). British Columbia: Babine Lake, $54^{\circ} 48^{\prime} \mathrm{N}$, 126 57'W, 8-VII-1974, Krajina \& al. s.n. (DAO); Cariboo district. end of Lac la Hache, 51 ${ }^{\circ} 50^{\prime} \mathrm{N}, 121^{\circ} 31^{\prime} \mathrm{W}, 2-$ VII-1961, Hitchick \& Muhlick 22084 (WTU); Chilcotin, $51^{\circ} 47^{\circ} \mathrm{N}, 122^{\circ} 38^{\prime} \mathrm{W}, 1-\mathrm{VIII}-1967$, Beil s.n. (DAO); E side of Sheep mountain near the Fire Warden's Cabin in the Elk River Valley $34 \mathrm{mi} \mathrm{N}$ of Natal. $49^{\circ} 10^{\prime} \mathrm{N}$, 115'13'W, 7-VII-1941, Weber 2342 (MO): Germansen Landing, Omineca River, $56^{\circ} 7^{\prime}$ N, $124^{\circ} 29^{\prime}$ W, 6-VII1940, McCabe 7720 (WTU): Kootenay, 50³9 N, $115^{\circ} 48^{\prime}$ W, 14-VII-1947, Eastham 15831 (DAO); Limestone Island off Louise Island, $52^{\circ} 55^{\prime} \mathrm{N}, 131^{\circ} 36^{\prime} \mathrm{W}, 9$.
VI-1964, Calder \& al. 34813 (DAO); Montney, $56^{\circ} 27^{\prime} \mathrm{N}, 120^{\circ} 51^{\prime} \mathrm{W}$, 5-IX-1934, Groh s.n. (DAO);

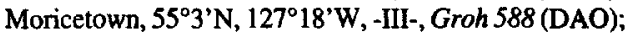
Queen Charlotte Islands, Limestone Island off Louise Island, 52 $59^{\prime} \mathrm{N}, 131^{\circ} 47^{\prime} \mathrm{W}, 9-\mathrm{VI}-1964$, Calder \& al. 34813 (MO WTU); Small Creek, $10 \mathrm{mi}$. N of Tete Jaune Cache, 52 ${ }^{\circ} 58^{\prime} \mathrm{N}, 119^{\circ} 25^{\prime} \mathrm{W}, 20$-VII-1971, Maze 1123 (WTU); Smithers, $54^{\circ} 46^{\prime} \mathrm{N}, 127^{\circ} 10^{\prime} \mathrm{W}, 12$-VIII-1939, Groh 542 (DAO); Summit lake, N of Prince George, $54^{\circ} 15^{\prime} \mathrm{N}, 122^{\circ} 38^{\prime} \mathrm{W}, 15-\mathrm{VI}-1963$, Auclair 352 (DAO); Topley, $54^{\circ} 30^{\prime} \mathrm{N}, 126^{\circ} 15^{\prime} \mathrm{W}, 21-\mathrm{VI}-1949$, Pillsbury 101 (DAO). Northwest Territories: Canada Tungsten Mine, Flat River, $61^{\circ} 58^{\prime} \mathrm{N}, 128^{\circ} 15^{\prime} \mathrm{W}, 20$-VII- 1967 , Cody \& Spicer 16350 (DAO); Hole-in-the-Wall Lake, $61^{\circ} 46^{\prime} \mathrm{N}, 127^{\circ} 16^{\prime} \mathrm{W}, 13-\mathrm{VII}-1977$, Scotter 24226 (DAO); Nahanni National Park, 61 ${ }^{\circ} 46^{\prime} \mathrm{N}, 127^{\circ} 11^{\prime} \mathrm{W}, 7$. VII-1976, Talbot 6011 (DAO). SASKATCHEWAN: Cypress Hills, Eastend, $49^{\circ} 31^{\prime} \mathrm{N}, 108^{\circ} 49^{\prime} \mathrm{W}, 8$-VIII- 1947 , Breitung 5475 (MO); Maple Creek, $49^{\circ} 54^{\prime} \mathrm{N}, 109^{\circ} 24^{\prime} \mathrm{W}, 14$ VI-1958, Boivin \& Perron 12095 (DAO). YUKON: Canol Rd., Rose-Lapie R. Pass, mile 105, 61 ${ }^{\circ} 36^{\prime} \mathrm{N}, 133^{\circ} 5^{\prime} \mathrm{W}$, 21-VII-1944, Porsild \& Breitung 10920 (WTU); Larsen Creek, $60^{\circ} 12^{\prime} \mathrm{N}, 125^{\circ} 32^{\prime} \mathrm{W}, 22$-VII-1977, Scobter 24747 (DAO); McArthur/Ethel Lake area, $63^{\circ} 4^{\prime} \mathrm{N}, 135^{\circ} 43^{\prime} \mathrm{W}$, 7-VIII-1987, Gallegher 659 (DAO); Pointed Mountain, $60^{\circ} 28^{\prime} \mathrm{N}, 123^{\circ} 50^{\circ} \mathrm{W}, 22-\mathrm{VII}-1974$, Lamont 607 (DAO).

USA. ARIZona: Apache Co., Springerville-Ford Apache road, $34^{\circ} 8^{\prime} \mathrm{N}, 109^{\circ} 17^{\prime} \mathrm{W}, 29$-VIII-1919, Eggleston 1576 (F); Coconino Co., Kendrick Peak, along Trail 22, $35^{\circ} 24^{\prime} \mathrm{N}, 111^{\circ} 51^{\prime} \mathrm{W}, 5$-VIII-1983, Ricketson 523 (MO); San Francisco Mountain, $35^{\circ} 20^{\prime} \mathrm{N}, 111^{\circ} 40^{\prime} \mathrm{W}$. 18-VIII1889, Knowlton 33 (MO); Shulz Pass near Flagstaff, $35^{\circ} 11^{\prime} \mathrm{N}, 111^{\circ} 39^{\prime} \mathrm{W}, 28$-VII-1922, Hanson 194 (MO). CALIFORNIA: El Dorado Co., The Firs, on the lincoln Highway near Echo Lake, $38^{\circ} 50^{\prime} \mathrm{N}, 120^{\circ} 2^{\prime} \mathrm{W}, 30$-VII-1919, Heller 13348 (MO); Fresno Co., Huntington Lake. $37^{\circ} 13^{\prime}$ N, $119^{\circ} 14^{\prime}$ W. 26-VII-1917. Grout 1145 (MO); Insulare Co., Hockelt's meadow, ${ }^{\circ} \mathrm{N},{ }^{\circ} \mathrm{W}, 19-\mathrm{VII}-1904$. Culberton 4357 (MO); Mariposa Co., Yosemite National Park, trail to Eagle Peak, $37^{\circ} 41^{\prime} \mathrm{N}, 19^{\circ} 47^{\prime}$ W. 12-VII1921, Thomas s.n. (F); Nevada Co, Donner Lake, $39^{\circ} 19^{\prime} \mathrm{N}$. $120^{\circ} 15^{\prime} \mathrm{W}, 6-\mathrm{VI}-1886$. Sonne 43 (MO); San Bemardino Co., Bear valley, San Berandino mountains. $34^{\circ} 15^{\prime} \mathrm{N}$, $116^{\circ} 50^{\prime} \mathrm{W}, 30$-VII-1902. Abrams 2831 (MO); Tulare Co., Sequoia National Park. Crescent Meadow, $36^{\circ} 33^{\prime} \mathrm{N}$. $118^{\circ} 44^{\prime} \mathrm{W}$, -VII-, Parks 5198 (UC). Colorado: Archuleta Co., Piedra, $37^{\circ} 13^{\prime} \mathrm{N}, 107^{\circ} 20^{\prime} \mathrm{W}$. 14-VII-1899, Baker 449

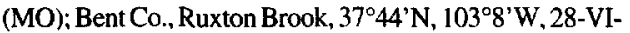
1901. Clements \& Clements 323 (MO); Boudler Co., Gregory Cañon, $40^{\circ} 0^{\prime} \mathrm{N}, 105^{\circ} 17^{\prime} \mathrm{W}, 16-\mathrm{VII}-1906$, Daniels 447 (MO); Canyon above Steads, Estes Park, $40^{\circ} 23^{\prime} \mathrm{N}$, 105'29'W, 3-VII-1912. Churchill s.n. (MO); Chaffee Co., near Chalk Creek, 5 mi above St. Elmo, $38^{\circ} 44^{\prime} \mathrm{N}, 106^{\circ} 3^{\prime} \mathrm{W}$, 13-VII-1936, Rollins I380 (MO); Clear Creek Co., Brookvale, Yankee Creek, $39^{\circ} 37^{\prime} \mathrm{N}, 105^{\circ} 25^{\prime} \mathrm{W}, 22$-VI-1918, Churchill s.n. (MO); Delta Co., Grand Mesa National Forest, $39^{\circ} 3^{\prime} \mathrm{N}, 107^{\circ} 48^{\prime} \mathrm{W}, 26-\mathrm{VII}-1981$, Siplivinsky 1755 (MA); Garfield Co., Trappers' Lake, $39^{\circ} 59^{\prime} \mathrm{N}, 107^{\circ} 13^{\prime} \mathrm{W}$, 29-VII-1933, Hermann 5393 (MO); Grand Co., Rocky Mountain National Park, Timber Creek, $40^{\circ} 16^{\prime} \mathrm{N}$, 106 ${ }^{\circ}$ W, 16-VIII-1997, Aedo 4416 (MA); Gunnison Co., Mineral point, $38^{\circ} 58^{\prime} \mathrm{N}, 107^{\circ} 6^{\circ} \mathrm{W}, 10-\mathrm{VII}-1887$, Trelease 
s.n. (MO); Huerfano Co., 3 mi below Blue Lakes Camp, Cucharas Creek W of La Veta, $37^{\circ} 53^{\prime} \mathrm{N}, 105^{\circ} 2^{\prime} \mathrm{W}, 29$-VII1936, Rollins 1285 (MO); Jackson Co., Rabbit Ears Pass, $40^{\circ} 23^{\prime} \mathrm{N}, 106^{\circ} 36^{\prime} \mathrm{W}, 20$-VII-1896, Baker 3100 (MO); Jefferson Co., Lookout Mountain, Golden, $39^{\circ} 43^{\prime} \mathrm{N}$, $105^{\circ} 14^{\prime} \mathrm{W}, 26-\mathrm{VII}-1917$, Johnston s.n. (MO); Lake Co., Leadville, $39^{\circ} 15^{\prime} \mathrm{N}, 106^{\circ} 17^{\prime} \mathrm{W}, 9-\mathrm{VII}-1886$, Trelease s.n. (MO); Larimer Co., Gray Mountain, $40^{\circ} 18^{\prime} \mathrm{N}, 105^{\circ} 17 \mathrm{~W}$, 23-VII-1885, Letterman s.n. (MO). IDAHO: Bannock Co., Mill Creek, Caribou National Forest, $42^{\circ} 57^{\prime} \mathrm{N}, 112^{\circ} 14^{\prime} \mathrm{W}$, 15-VII-1952, Baker 9315 (WTU); Bear Lake Co., Sharon, $42^{\circ} 21^{\prime} \mathrm{N}, 111^{\circ} 28^{\prime} \mathrm{W}, 15$-VII-1952, Baker 9315 (WTU); Bingham Co., Blackfoot Mountains, Wolverine Creek, $43^{\circ} 15^{\prime} \mathrm{N}, 112^{\circ} 1$ 'W, 14-VII-1971, Holmgren \& Marttala 5406 (WTU); Bonneville Co., Caribou Mountain, $43^{\circ} 5^{\prime} \mathrm{N}$, $111^{\circ} 18^{\prime} \mathrm{W}, 17-\mathrm{VII}-1923$, Payson \& Armstrong 3523 (MO); Clark Co., Beaver Creek $3 \mathrm{mi}$ above Spencer, $44^{\circ} 24^{\prime} \mathrm{N}$, $112^{\circ} 11^{\prime} \mathrm{W}, 24-\mathrm{VI}-1939$, Cronquist 1307 (MO); Clearwater Co., Headquarters, $46^{\circ} 37^{\prime} \mathrm{N}, 115^{\circ} 48^{\prime} \mathrm{W}, 17-\mathrm{VII}-1959$, Baker 15669 (WTU); Fremont Co., Henry Lake, 44 $35^{\prime} \mathrm{N}$, $111^{\circ} 21^{\prime} \mathrm{W}, 10-\mathrm{VII}-1920$, Payson \& Payson 1948 (MO); Idaho Co., Pete King Creek on Lochsa River Canadian, Nez Perce National Forest, $46^{\circ} 9^{\prime} \mathrm{N}, 115^{\circ} 35^{\prime} \mathrm{W}, 31-\mathrm{V}-1936$, Constance \& Rollins 1677 (MO); Nez Perce Co., near Beaver Canon, $46^{\circ} 23^{\prime} \mathrm{N}, 116^{\circ} 48^{\prime} \mathrm{W}, 11-\mathrm{VIII}-1892$, Mulford s.n. (MO); Twin Falls Co., South Hills, Fourth Fork Rock Creek, Magic Mountain Ski Area, $42^{\circ} 11^{\prime} \mathrm{N}$, $114^{\circ} 17^{\prime} \mathrm{W}, 11-\mathrm{VII}-1972$, Holmgren 6181 (WTU); Valley
Co., South Fork of the Salmon River at South Fork Camp, $44^{\circ} 30^{\prime} \mathrm{N}, 116^{\circ} 2^{\prime} \mathrm{W}, 19-\mathrm{VII}-1954$, Baker 11925 (WTU). MontANA: Beaverhead Co., along Price-Peet Road, ca 0.5 mi W of Pete Creek Divide, $44^{\circ} 32^{\prime} \mathrm{N}, 112^{\circ} 4^{\prime} \mathrm{W}, 12$-VII1979, Lowry 2490 (MO); Carbon Co., Red Lodge, on Beartooth plateau, $45^{\circ} 11^{\prime} \mathrm{N}, 109^{\circ} 14^{\prime} \mathrm{W}, 5$-VII-1954, Booth 54480 (WTU); Cascade Co., $20.9 \mathrm{mi} \mathrm{N}$ of Neihart along Highway $89,46^{\circ} 59^{\prime} \mathrm{N}, 110^{\circ} 44^{\prime} \mathrm{W}, 3$-VII-1963, Gillett \& Mosquin 11840 (MO); Deer Lodge Co., near Low Pass, $46^{\circ} 12^{\prime} \mathrm{N}, 113^{\circ} 11^{\prime} \mathrm{W}, 24-\mathrm{VI}-1860$, Hayden s.n. (MO); Fergus Co., 4 mi up Half Moon Canyon, Big Snowy mts., $46^{\circ} 49^{\prime} \mathrm{N}, 109^{\circ} 16^{\prime} \mathrm{W}, 5$-VII-1945, Hitchcock \& Muhlick 11969 (MO); Gallatin Co., Bozeman, Bear Canon, $45^{\circ} 37^{\prime} \mathrm{N}, 110^{\circ} 5^{\prime} \mathrm{W}, 7-\mathrm{VII}-1905$, Blankinship 103 (MO); Granite Co., Rattler Gulch Road, $46^{\circ} 41^{\prime} \mathrm{N}, 113^{\circ} 13^{\prime} \mathrm{W}, 20-$ VII-1976, Lackschewi s.n. (WTU); Lewis \& Clark Co., W side of McDonald Pass, $47^{\circ} 0$ 'N, $112^{\circ} 31^{\prime} \mathrm{W}, 29-\mathrm{VI}-1945$, Hitchcock \& Muhlick 11739 (MO); Madison Co., on Bear Trap Canyon Trail, $45^{\circ} 32^{\prime} \mathrm{N}, 111^{\circ} 35^{\prime} \mathrm{W}, 18-\mathrm{VII}-1979$, Lowry 2542 (MO); Meagher Co., Lewis and Clark national Forest, Jumping Creek Campground, $46^{\circ} 46^{\circ} \mathrm{N}, 110^{\circ} 46^{\prime} \mathrm{W}$, 3-VIII-1994, Merello \& Galloway 1044 (MO); Park Co., $14 \mathrm{mi}$ SE of Cooke City, $45^{\circ} 1^{\prime} \mathrm{N}, 109^{\circ} 56^{\prime} \mathrm{W}, 19-\mathrm{VII}-1969$, Zaczakowski 1058 (MO); Pondera Co., SW Glacier Park, Marias Pass, $48^{\circ} 19^{\prime} \mathrm{N}, 113^{\circ} 21^{\prime} \mathrm{W}, 7-\mathrm{VII}-1948$, Hitchcock 18127 (WTU); Powell Co., $10 \mathrm{mi}$ W of Lincoln, $46^{\circ} 56^{\circ} \mathrm{N}$, $112^{\circ} 55^{\prime} \mathrm{W}, 1-\mathrm{VII}-1948$, Hitchcock 17849 (WTU); Silver Bow Co., near Butte, $45^{\circ} 59^{\prime} \mathrm{N}, 112^{\circ} 32^{\prime} \mathrm{W}, 1893$, Moore
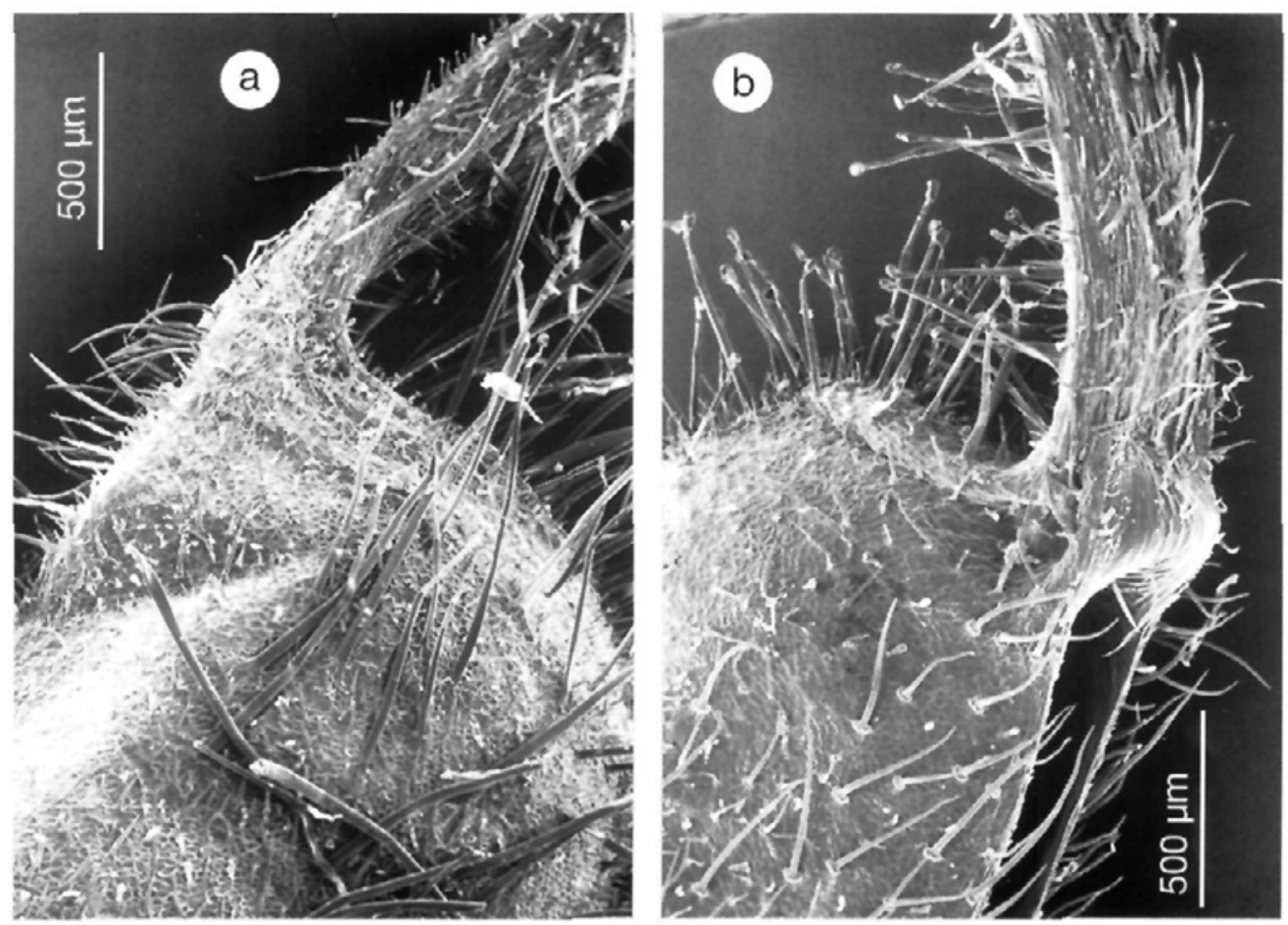

Fig. 30.-Mericarps SEM photomicrographs of: a, Geranium erianthum [Calder 14736 (DAO)], and b, G. richardsonii [Turner 2145 (DAO)]. 


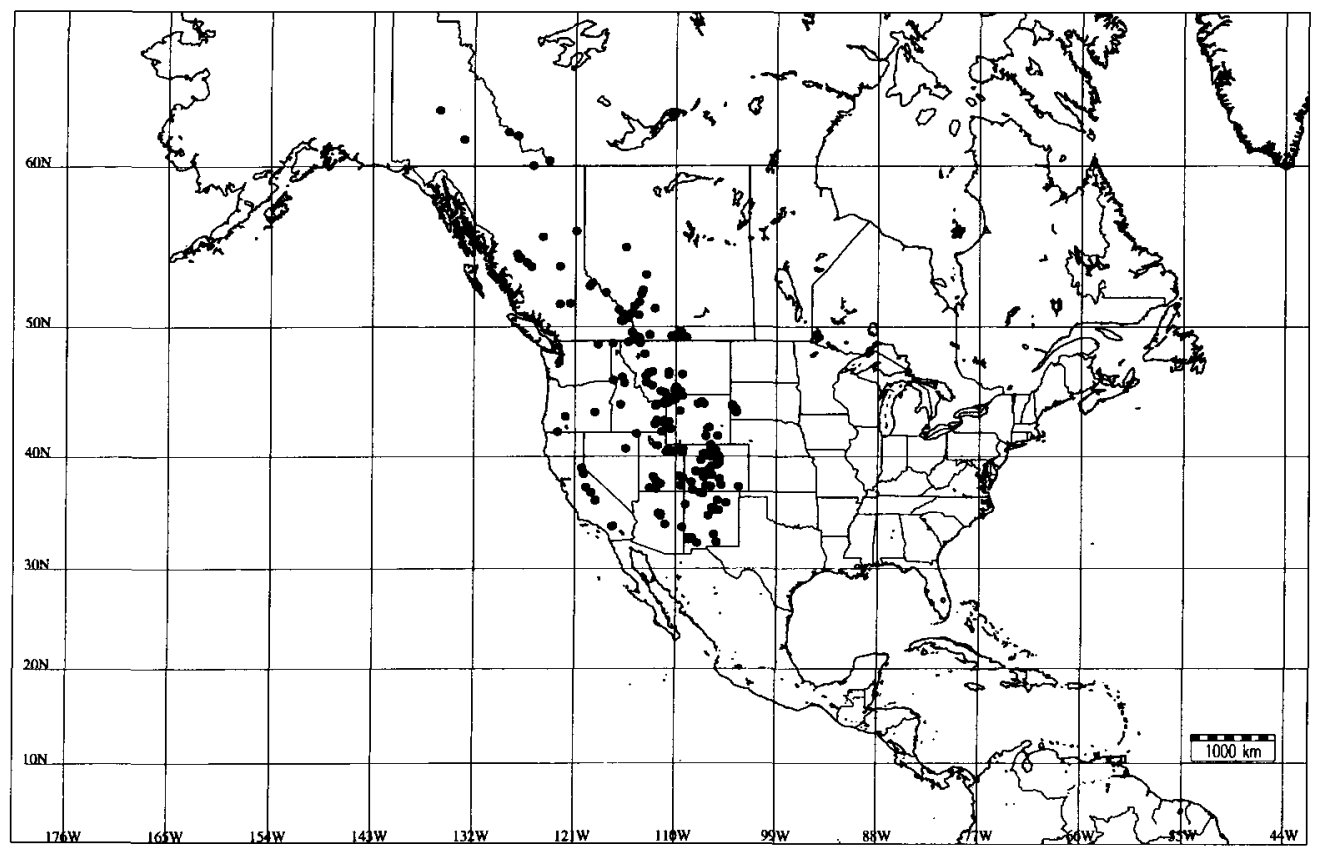

Fig. 31.-Distribution of Geranium richardsonii.

s.n. (MO); Stillwater Co., Independence, head of Boulder Creek, Absaroka Nat. Forest, $45^{\circ} 31^{\prime}$ N, $109^{\circ} 27^{\prime}$ W, 7-VIII1945, Hitchcock \& Muhlick 13335 (MO). NeVADA: Elko Co., Camp Halleck, $40^{\circ} 57^{\prime} \mathrm{N}, 115^{\circ} 27^{\prime} \mathrm{W}$, -VIII-, Palmer 56 (F). New Mexico: Bernalillo Co., Sandia Mountains, Bal-

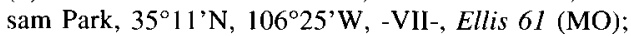
Catron Co., Mogollon mts., 33'13'N, $108^{\circ} 31^{\prime} \mathrm{W}$, -VIII-, Rusby 59 (MO); Colfax Co., Cimarron River, $5 \mathrm{mi}$ E of Eagle Nest, 36 ${ }^{\circ} 19^{\prime}$ N, 104 $29^{\prime} \mathrm{W}, 15-\mathrm{IX}-1944$, Lucas 227 (F); Grant Co., Pinos Altos Mountains, Little Creek, 33 $12^{\prime} \mathrm{N}$, 108'12'W, 23-IX-1987, Shelton 196 (NY); Lincoln Co., White Mountains, $33^{\circ} 29^{\prime} \mathrm{N}, 105^{\circ} 50^{\prime} \mathrm{W}, 9$-VIII-1897, Wooton 302 (MO); McKinley Co., Chuska mts., $36^{\circ} 10^{\prime} \mathrm{N}$, $108^{\circ} 55^{\prime} \mathrm{W}$, -VII-, Walco 159 (MO); Otero Co., Sacramento, 32 $47^{\circ} \mathrm{N}, 105^{\circ} 33^{\prime} \mathrm{W}, 6-\mathrm{VIII}-1969$, Conley s.n. (NMC);

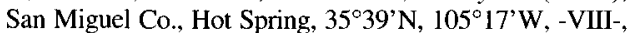
Snow s.n. (MO); Santa Fe Co., vicinity of Santa Fe, Lake Peak, $35^{\circ} 47^{\prime} \mathrm{N}, 105^{\circ} 46^{\prime} \mathrm{W}, 15-\mathrm{VII}-1926$, Arsène \& Benedict 16055 (MO); Sierra Co., Taylor Creek, 32 $42^{\prime} \mathrm{N}$, 107³8'W, 14-VIII-1982, Spellenberg \& al. 6609 (NMC); Socorro Co., Mogollon mts, on Mogollon Creek, $33^{\circ} 2^{\prime} \mathrm{N}$, $108^{\circ} 31^{\prime}$ W, 23-VII-1903, Metcalfe 301 (MO); Taos Co., Sangre de Cristo Mountains, Lake Fork Canyon, $36^{\circ} 33^{\prime} \mathrm{N}$, 105 $26^{\prime}$ 'W, 22-VIIl-1973, Holmgren \& Holmgren 7360 (WTU). OREGON: Harney Co., $1 \mathrm{mi} \mathrm{S}$ of Calamity Guard Station, $43^{\circ} 55^{\prime} \mathrm{N}, 118^{\circ} 49^{\prime} \mathrm{W}, 26-\mathrm{VI}-1953$, Mc Cutcheon 620 (WVA); Jackson Co., High Cascades east of Medford, $42^{\circ} 19^{\prime} \mathrm{N}, 122^{\circ} 52^{\prime} \mathrm{W},-\mathrm{VI}-$, Heckner s.n. (WTU); Klamath Co., Crater National Forest, Cascade Mountains, Cherry Creek, $43^{\circ} 34^{\prime} \mathrm{N}, 122^{\circ} 2^{\prime} \mathrm{W}$, - VIII-, Rose 1148 (MO); Sheep Mountain, near Fire Warden's Cabin, 34 mi N of Natal, $49^{\circ} 51^{\prime} \mathrm{N}, 114^{\circ} 48^{\prime} \mathrm{W}, 7-\mathrm{VII}-1941$, Weber 2342 (WTU). SOUTH DAKOTA: Estes, $44^{\circ} 10^{\prime} \mathrm{N}, 103^{\circ} 29^{\prime} \mathrm{W}, 23$-VII-1917, Visher 1544 (F); Lawrence Co., Deadwood, 442'N, $103^{\circ} 43^{\prime}$ W, 23-VII-1913, Rydberg 168 (MO); Pennington Co., Black Hills National Forest, $10 \mathrm{mi} \mathrm{S}$ of Keystone, $43^{\circ} 53^{\prime} \mathrm{N}, 103^{\circ} 25^{\prime} \mathrm{W}, 2-\mathrm{VII}-1966$, Croat 2435 (MO). Utah: Daggett Co., Carter Creek Canyon, $40^{\circ} 53$ 'N, 109'35'W, 11-VII-1933, Hermann 4796 (MO); Duchesne Co., Chain Lakes Trail, 6 mi SE mt Emmons, 4042'N, $110^{\circ} 15^{\prime} \mathrm{W}, 23-\mathrm{VII}-1933$, Hermann 5241 (MO); Garficld Co., Bryce Canyon National Park, Sheep Creek, 37 $33^{\prime} \mathrm{N}$, $112^{\circ} 7^{\prime}$ W, 2-VII-1993, Foster 10870 (MO); Grand Co., La Sal Mts., 38³8'N, $109^{\circ} 35^{\prime}$ W, 31-VII-1924, Payson \& Payson 4093 (MO); Iron Co., Cedar Breaks, above the Breaks, 37³8'N, 112 50'W, 19-VII-1929, Mathias 697 (MO); Piute Co., 2 mi NW junction T29S, R3W, 38 $15^{\prime}$ N, $112^{\circ} 7^{\prime}$ W, 28-VII-1976, Welsh \& al. 14110 (MO); San Juan Co., Blue Lake, La Sal Mountains, 38 28'N, $109^{\circ} 12^{\prime} \mathrm{W}$, 30-VI-1967, Pederson 101 (MO); Sevier Co., Fish Creek, $38^{\circ} 35^{\prime}$ N, $112^{\circ} 24^{\prime} \mathrm{W}$, - VII-, Garret 7550 (MO); Summit Co., Fire Lake, $40^{\circ} 40^{\prime} \mathrm{N}, 111^{\circ} 1^{\prime} \mathrm{W}, 19-\mathrm{VII}-1991$, Windham 9I-200 (MO); Uintah Co., Diamond Mountains, 40 $36^{\prime} \mathrm{N}$, $109^{\circ} 14^{\prime} \mathrm{W}, 5$-VIII-1912, [illegible] (MO); Weber Co., Ogden, $41^{\circ} 13^{\prime} \mathrm{N}, 111^{\circ} 58^{\prime} \mathrm{W}, 2-\mathrm{VIII}-1887$, Tracy 612 (MO). Wroming: Albany Co., Pole Creek, $42^{\circ} 0^{\prime} \mathrm{N}, 105^{\circ} 25^{\prime} \mathrm{W}, 1$ VII-1895, Nelson 1403 (MO); Big Horn Co., $0.7 \mathrm{mi} \mathrm{S}$ of Granite Pass, Big Horn mts., 44 $34^{\prime} \mathrm{N}, 107^{\circ} 32^{\prime} \mathrm{W}, 17-\mathrm{VIII}-$ 1968, Lewis 7072 (MO); Carbon Co., Medicine Bow Range, Arlington, $41^{\circ} 59^{\prime} \mathrm{N}, 106^{\circ} 40^{\prime} \mathrm{W}, 15$-VII-1988, $\mathrm{Ni}$ eto Feliner \& Lansac 2090GN (MA); Lincoln Co., Grand Canyon of Snake River, Bailey Lake, $43^{\circ} 10^{\prime} \mathrm{N}, 110^{\circ} 44^{\prime} \mathrm{W}$, 
9-VII-1932, Williams 824 (MO); Natrona Co., Graden Brook, N slope of Casper Mt, $42^{\circ} 43^{\prime} \mathrm{N}, 106^{\circ} 19^{\prime} \mathrm{W}, 8$-VII1933, Hermann 4625 (MO); Park Co., Carzi Woman Creek, Shoshone National Forest, $44^{\circ} 0^{\circ} \mathrm{N}, 109^{\circ} 30^{\prime} \mathrm{W}, 11-$ VII-1937. Williams \& Williams 3512 (MO); Sheridan Co., Rapid Creek Park, $44^{\circ} 43^{\prime}$ N, $107^{\circ} 8^{\prime}$ W, -VII-, Pammel \& Stanton s.n. (MO); Sublette Co., $25 \mathrm{mi}$ W Big Piney, N side of Middle Piney Lake, $42^{\circ} 35^{\circ}$ N, $110^{\circ} 34^{\prime}$ W, 31-VIII-1947, Meyer \& Meyer 2404 (MO); Teton Co., Firehole River, 3

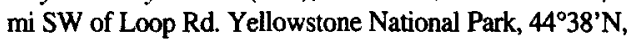
$110^{\circ} 51^{\prime} \mathrm{W}, 27-\mathrm{VI}-1960$, Venrick 396 (MO).

17.Geranium viscosissimum Fisch. \& C.A. Mey. in C.A. Mey., Index Sem. Hort. Petrop. 11, Suppl.: 18 (1846) [Dic. 1846, probably 1847$]$

Ind. loc.: "Hab. in America septentrionali occidentali (Lindl)"

Typus: North America [cultivated at LE from seeds collected by Lindley] (lectotype, here designated, LE!)

$=$ G. nervosum Rydb. in Bull. Torrey Bot. Club 28: 34 (1901)

G. viscosissimum var. nervosum (Rydb.) C.L. Hitchc. in C.L. Hitchc. \& al., Vasc. Pl. Pacific Northw. 3: 383 (1961)

G. viscosissimum subsp. nervosum (Rydb.) W.A. Weber in Phytologia 51: 374 (1982)

Ind. loc.: "Wyoming: Fish Creek, Teton Forest Reserve, 1897, F. Tweedy, 494 (type)"

Typus: USA. Wyoming, Fish Creek, Teton Forest Reserve, 1897, Tweedy 494 [lectotype, designated by JONES \& JONES (1943: 24), NY!]

= G. strigosum Rydb. in Bull. Torrey Bot. Club 29: 243 (1902), nom. illeg., non Burm. f. 1768

G. strigosior H. St. John, Fl. Southeast Wash. \& Idaho: 243 (1937), nom. nov.

Ind. loc.: "Wyoming: Copperton, 1901, F. Tweedy, 4591 (type in herb. N.Y. Bot. Gard."

Typus: USA. Wyoming, Copperton, June 1901, Tweedy 4591 (holotype, NY!)

$=G$. ornatum A. Nelson ex R. Knuth in Engl., Pflanzenr. IV.129 (Heft 53): 117 (1912), nom. nud., pro syn.

= G. viscosissimum var. album Suksd. in Werdenda 1: 24 (1923)
G. viscosissimum f. album (Suksd.) H.St. John in Proc. Biol. Soc. Wash. 41: 195 (1928)

Ind. loc.: "Bei Spangle, nördlich davon, in Spokane County, 29. Juni 1916, Nr. 8710" Typus: USA. Washington, Spokane Co., Spangle, 29 Jun. 1916, Suksdorf 8710 (lectotype, here designated, MO!, isolectotypes, BM! GH ILL K! NY UC)

$=$ G. attenuilobum G.N. Jones \& F.L. Jones in Rhodora 45: 40 (1943)

Ind. loc.: "California: Jess Valley, Warner Mountains, Modoc Co., July 24, 1925, F.H. Frost 113"

Typus: USA. California, Modoc Co., Jess Valley, Warner Mountains, 24 July 1925, Frost 113 (holotype, GH!; isotype, UC)

Herbs perennial, (30-)50-90 cm tall. Rootstock 12-22 mm diam., vertical, not tuberculate, not turnip-shaped. Stem erect, usually solitary, with patent to retrorse, eglandular hairs $0.3-1.7 \mathrm{~mm}$ long, and usually patent, glandular hairs $0.1-0.8 \mathrm{~mm}$ long. Basal leaves in a persistent rosette; lamina $6.8-15 \times 7.1-16$ $\mathrm{cm}$, polygonal in outline, cordate, palmatifid (divided for 0.75-0.87 of its length), pilose, with appressed, eglandular (and sometimes glandular) hairs; segments 5-7, rhombic, 7-15 $\mathrm{mm}$ at the base, 9-13-lobed in distal half (ratio main-sinus length of the middle segment / middle-segment length $=0.17-0.28(-0.4)$ ); cauline leaves opposite; petioles to $35 \mathrm{~cm}$ long, with patent to retrorse, eglandular hairs $0.3-1.1 \mathrm{~mm}$ long, and sometimes, patent glandular hairs 0.3-0.9 mm long; stipules $11-15 \times 2-3 \mathrm{~mm}$, lanceolate, with eglandular (and sometimes glandular) hairs on both sides and on the margin. Inflorescence a dichasial cyme; cymules 2-flowered, solitary or in aggregates at the top of each branch; peduncles $(0-) 1-13 \mathrm{~cm}$ long, with patent, glandular hairs $0.3-0.9 \mathrm{~mm}$ long and patent to retrorse, eglandular hairs 0.2-1.9 $\mathrm{mm}$ long, sometimes lacking glandular hairs; bracteoles 5-9 × 1-1.5 mm, linear, with eglandular hairs on both sides and on the margin; pedicels $0.6-3.4 \mathrm{~cm}$ long, with patent, glandular hairs $0.2-0.9 \mathrm{~mm}$ long and patent to retrorse, eglandular hairs $0.1-1.9 \mathrm{~mm}$ long, rarely lacking glandular hairs; pedicel and peduncle to- 


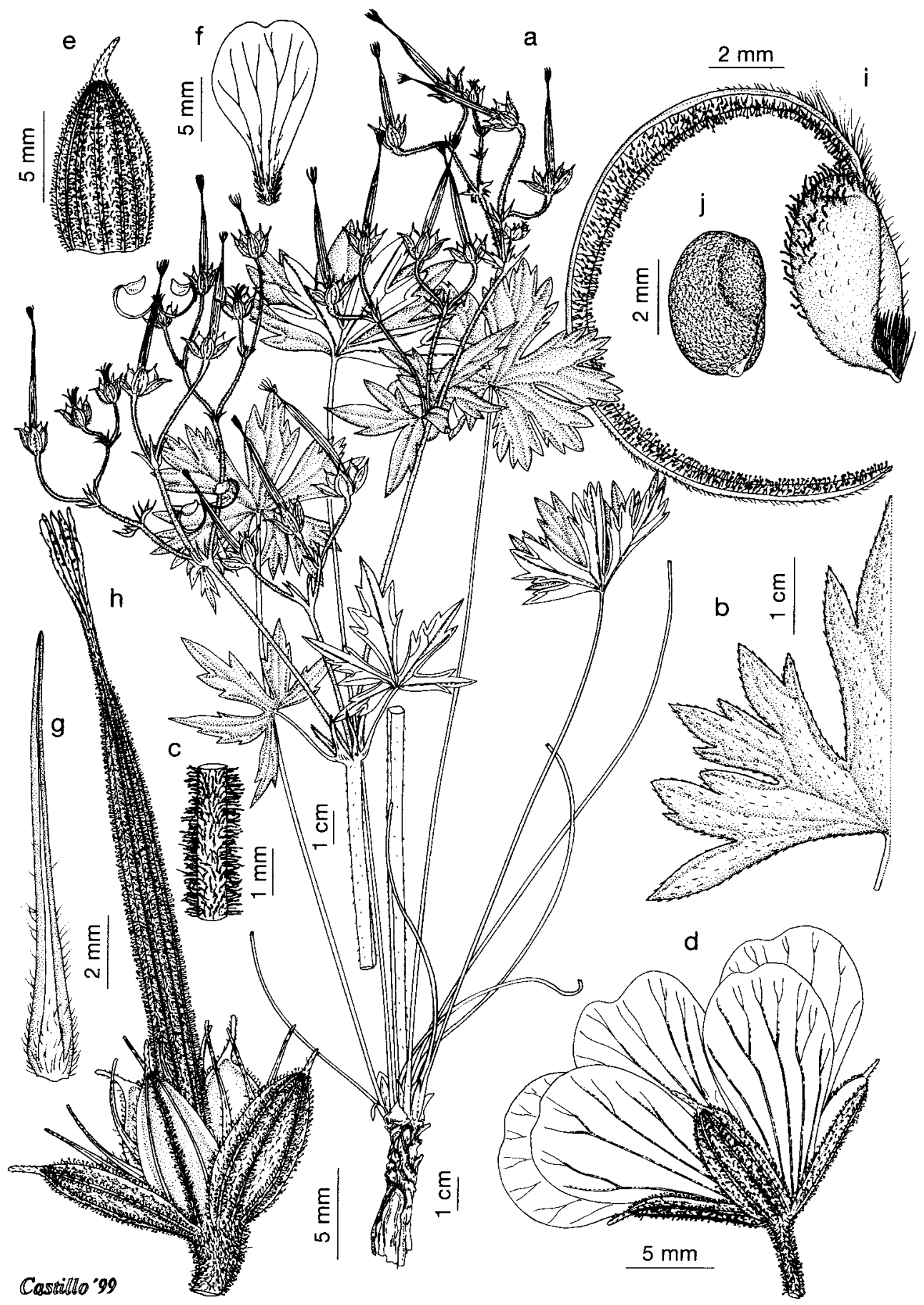

Fig. 32.-Geranium viscosissimum: a, Habit; b, Leaf; c, Peduncle; d, Flower; e, Sepal; f, Petal; g, Staminal filament; h, Fruit; i, Mericarp; j, seed. [a, e, g-j based on Andrews 662 (OSC), b-d, f based on Wagner 1447 (OSC)]. 
gether often overtopping the subtending leaf. Sepals 8-11 $\times 3-4.5 \mathrm{~mm}$ (ratio pedicel length / sepal length $=1.1-3.7$ ), smooth, not accrescent, 3-5-nerved, with mucro 1-2 mm long, with scarious margins $0.2-0.3 \mathrm{~mm}$ wide, with antrorse, appressed, eglandular hairs 0.2-0.6 $\mathrm{mm}$ long and patent, glandular hairs 0.3-0.6 $\mathrm{mm}$ long on the abaxial side, usually hairy on the base of the adaxial side. Petals $17-20 \times 9$ $15 \mathrm{~mm}$ (ratio petal length / petal width $=1.3$ 1.8), erect-patent, entire, without claw, hairy on 1/3-1/2 of their adaxial surface and on the base of the abaxial one, ciliate on the basal margin, purplish. Stamens 10, both whorls bearing anthers; filaments $8-9 \mathrm{~mm}$ long, not exserted, lanceolate, pilose on the abaxial side, ciliate on the proximal half, with hairs up to 1.8 $\mathrm{mm}$ long; anthers $2-2.5 \times 1 \mathrm{~mm}$. Nectaries with a tuft of hairs at the top, dorsally glabrous. Gynoecium 11-13 mm long. Fruit 33-41 mm long, erect when immature; mericarps 4.5-5.5 $\times 2.5$ $3 \mathrm{~mm}$, without a strand of fibres, smooth (with 2 transversal veins at the apex), with longitudinal rib, without basal beak, with a basal callus, with \pm patent, eglandular hairs $0.2-0.4 \mathrm{~mm}$ long, and glandular hairs $0.3-0.8 \mathrm{~mm}$ long, brownish; rostrum $25-29 \mathrm{~mm}$ long, with a narrowed apex 3-4 mm long, with \pm patent, eglandular hairs 0.1-0.2 mm long, and glandular hairs $0.5-0.9 \mathrm{~mm}$ long; stigmatic remains 3-6 $\mathrm{mm}$ long (ratio fruit length / stigmatic remains length $=6-8.2$ ), with 5 glabrous lobes. Seeds $3.5-4 \times 2.5 \mathrm{~mm}$, reticulate, brownish; hilum $1 / 4$ as long as the perimeter. Cotyledons entire.

Flowering April-September. Coniferous forests (also in aspen or maple woods), wet meadows, grassy slopes or sandy banks; 300$3200 \mathrm{~m}$. Western Canada, Northwestern U.S.A., Southwestern U.S.A. \& North-Central U.S.A. Fig. 33.

\section{Illustrations. Fig. 1b, 32.}

The presence of glandular hairs also varies in this species. Forms with eglandular stems, and more rarely with eglandular peduncles and pedicels, appear frequently towards the southem part of its area (Arizona, New Mexico). However, there are all possible transitions to glandular organs, suggesting that such eglandular speci- mens do not deserve taxonomic recognition. Geranium strigosum was described to included these eglandular specimens. Furthermore, leaf shape and proportion of petal surface bearing hairs varies considerably but independently of glandular indumentum presence. Geranium attenuilobum was described to include forms with petals bearing hairs on more than one-third.

The specimens supporting the records of $G$. viscosissimum from New Mexico, (MARTIN \& HUTCHINS 1980: 1123; KARTESZ 1998) have not been studied.

\section{Representative specimens examined}

Canada. Alberta: Cardston, Milk River Ridge, $49^{\circ} 15^{\prime} \mathrm{N}, 112^{\circ} 30^{\prime} \mathrm{W}, 7$-VIII-1950, Dore \& Breitung 12383 (DAO); Carway, $49^{\circ} 1{ }^{\prime} \mathrm{N}, 113^{\circ} 22^{\prime} \mathrm{W}, 2$-VIII-1950, Dore \& Breitung 12266 (DAO); Coleman, 52 ${ }^{\circ} 7^{\prime} \mathrm{N}, 116^{\circ} 55^{\prime} \mathrm{W}, 3-$ VIII-1959, Russell s.n. (DAO); Cypress Hills, Grayburn Butte, $49^{\circ} 34^{\prime} \mathrm{N}, 110^{\circ} 8^{\prime} \mathrm{W}, 26-\mathrm{VII}-1947$, Breitung 5078 (MO); Highwood River, Forestry Trunk Road, $50^{\circ} 49^{\prime} \mathrm{N}$, 113ํㄱ' W, 20-VII-1977, Kojima s.n. (DAO); KananaskisColeman Rd., 51 ${ }^{\circ} 4^{\prime} \mathrm{N}, 115^{\circ} 8^{\prime} \mathrm{W}, 15-\mathrm{VII}-1953$ (DAO); Milk River, $49^{\circ} 7^{\prime}$ N, $112^{\circ} 7^{\prime}$ 'W, 26-VI-1952, Boivin \& Alex s.n. (DAO); Pincher Creek Bridge, $49^{\circ} 29^{\prime} \mathrm{N}, 113^{\circ} 57^{\prime} \mathrm{W}, 6-$ VI-1963, Taylor \& Sherk 4825 (DAO); SW side of Sarcee Reservation, SW side of Calgary, $51^{\circ} 1^{\prime} \mathrm{N}, 114^{\circ} 32^{\prime} \mathrm{W}, 3-$ IX-1969, D'Arcy 3726 (MO); Waterton lake National Park, $4^{\circ} 5^{\prime} \mathrm{N}, 113^{\circ} 52^{\prime} \mathrm{W}, 24-\mathrm{VII}-1968$, Bailey 68 (DAO); Waterton Lakes, $43^{\circ} 29^{\prime} \mathrm{N}, 107^{\circ} 14^{\prime} \mathrm{W}, 23-\mathrm{VII}-1940$, Hart 8 (DAO). British Columbia: $30 \mathrm{mi} \mathrm{NW}$ of Clinton, $51^{\circ} 20^{\prime} \mathrm{N}, 122^{\circ} 2^{\prime} \mathrm{W}, 23-\mathrm{VI}-1956$, Calder \& al. 17828

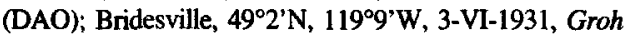
s.n. (DAO); Chilcotin, $51^{\circ} 48^{\prime} \mathrm{N}, 122^{\circ} 39^{\prime} \mathrm{W}$, -II-, Beil $68^{-}$ 07-12 (DAO); Crowsnest Pass, $49^{\circ} 38^{\prime} \mathrm{N}, 114^{\circ} 41^{\prime} \mathrm{W}, 4-$ VII-1947, Eastham 15731 (DAO); Falkland, 50 $30^{\prime} \mathrm{N}$, $119^{\circ} 33^{\prime} \mathrm{W}, 10-\mathrm{VI}-1955$, Lindsay \& Woodbury 1027 (DAO); Kamloops, $49^{\circ} 56^{\prime} \mathrm{N}, 121^{\circ} 36^{\prime} \mathrm{W}, 1$-VI-1953, Calder \& Savile 8490 (DAO); Lumbdon Lake East, $50^{\circ} 8^{\prime} \mathrm{N}, 120^{\circ} 36^{\prime} \mathrm{W}, 28-\mathrm{V}-1995$, Dickson \& Fairbarns 6815 (DAO); Merritt, 507’ N, $120^{\circ} 46^{\circ} \mathrm{W}, 29-\mathrm{V}-1956$, Calder \& Parmelee 1682I (DAO); Nigger Lake. $49^{\circ} 26^{\prime} \mathrm{N}$, $115^{\circ} 56^{\prime} \mathrm{W}, 14-\mathrm{VII}-1958$, Taylor \& Ferguson 2766 (DAO); Riske Creek to Hanceville, 51 ${ }^{\circ} 58^{\prime} \mathrm{N}, 122^{\circ} 31^{\prime} \mathrm{W}, 22$-VII1956, Calder \& Parmelee 19272 (DAO); Rock Creek, $49^{\circ} 3^{\prime} \mathrm{N}, 119^{\circ} 0^{\prime} \mathrm{W}, 30-\mathrm{VI}-1975$, Hainault 7342 (DAO); Sage Creek Flathead, $51^{\circ} 43^{\prime} \mathrm{N}, 11^{\circ} 22^{\prime} \mathrm{W}, 27-\mathrm{VII}-1957$, Eastham 618 (DAO); Williams Lake region, Big Lake, $52^{\circ} 23^{\prime} \mathrm{N}, 121^{\circ} 50^{\prime} \mathrm{W}, 10-\mathrm{VI}-1934$, Murie 1193 (MO). SASKATCHEWAN: Cypress Hills, Battle Creek Ranger Station, $49^{\circ} 34^{\prime} \mathrm{N}, 109^{\circ} 53^{\prime} \mathrm{W}, 13-\mathrm{VII}-1947$, Breitung 4759 (DAO); Kandahar, $51^{\circ} 46^{\prime} \mathrm{N}, 104^{\circ} 21^{\prime} \mathrm{W}$, Breitung 1637 (DAO); Killdeer, $49^{\circ} 7^{\prime} \mathrm{N}, 106^{\circ} 21^{\prime} \mathrm{W}, 26-\mathrm{VII}-1966$, Hudson 2335 (DAO); Rivière Bataille, $52^{\circ} 33^{\prime} \mathrm{N}, 106^{\circ} 10^{\prime} \mathrm{W}, 3-$ VIII-1858, Bourgeau s.m. (P).

USA. California: Modoc Co., Jess Valley, Wamer Mountains, $41^{\circ} 14^{\prime} \mathrm{N}, 120^{\circ} 20^{\circ} \mathrm{W}, 24-\mathrm{VII}-1925$, Frost 113 (GH); Siskiyou Co., Quartz Valley, $41^{\circ} 36$ 'N, $122^{\circ} 58^{\prime} \mathrm{W}$, 
6-VI-1910, Butler 1458 (MO). COLORADo: Garfield Co., on Grizzly Creek, $39^{\circ} 33^{\prime} \mathrm{N}, 107^{\circ} 15^{\prime} \mathrm{W}, 19-\mathrm{VII}-1896, B a k$ er s.n. (MO); Gunnison Co., Gunnison Watershed, $38^{\circ} 32^{\prime}$ N, $106^{\circ} 55^{\prime} \mathrm{W}, 26-\mathrm{VII}-1901$, Baker 622 (MO). IDAHO: Ada Co., Boise, $43^{\circ} 36^{\prime} \mathrm{N}, 116^{\circ} 12^{\prime} \mathrm{W}, 18-\mathrm{VI}-1910$,

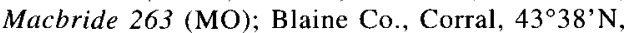
$14^{\circ} 2^{\prime} \mathrm{W}, 28-\mathrm{VI}-1916$, Macbride \& Payson 2931 (MO); Boise Co., Clear Creek, 444'N, $115^{\circ} 36^{\prime} \mathrm{W}, 4$-VII-1911, Clark 78 (MO); Bonner Co., S end of Lake Pend Oreille, $48^{\circ} 9^{\prime} \mathrm{N}, 116^{\circ} 20^{\prime} \mathrm{W},-\mathrm{VI}-$, Leiberg s.n. (MO); Clark Co., Spencer, $44^{\circ} 21^{\prime} \mathrm{N}, 112^{\circ} 11^{\prime} \mathrm{W}, 23-\mathrm{VI}-1939$, Cronquist 1255 (MO); Elmore Co., Trinity, $43^{\circ} 37^{\prime} \mathrm{N}, 115^{\circ} 22^{\prime} \mathrm{W}, 9-$ VIII-1910, Macbride 558 (MO); Idaho Co, along Clearwater River opposite Kamiah, $46^{\circ} 1$ 'N, $115^{\circ} 53^{\prime} \mathrm{W}, 6$-VI1937, Meyer 924 (MO); Kootenai Co., Lake Coeur d'Alene, 47 $42^{\prime} \mathrm{N}, 116^{\circ} 57^{\prime} \mathrm{W}, 3-\mathrm{VIII}-1926$, Epling \& Hauch 10038 (MO); Latah Co., Moscow, 46 $43^{\prime} \mathrm{N}$, $116^{\circ} 59^{\prime} \mathrm{W}, 13-\mathrm{VII}-1943$, Murley 1745 (MO); Nez Perce Co., Potlatch Creek, $46^{\circ} 28^{\prime} \mathrm{N}, 11^{\circ} 45^{\prime} \mathrm{W}, 29-\mathrm{V}-1899$, Trelease 4366 (MO); Owyhee Co., Silver City, 431'N, $116^{\circ} 43^{\prime} \mathrm{W}, 22-\mathrm{VI}-1911$, Macbride 959 (MO); Twin Falls Co., House Creek, $42^{\circ} 11^{\prime}$ N, $114^{\circ} 54^{\prime} \mathrm{W}, 30-\mathrm{VI}-1912$, Nelson \& Macbride 1819 (MO); Valley Co., Sagebrush plain $10 \mathrm{mi} \mathrm{S}$ of McCall, 45'16'N, 114²6'W, 26-VI-1946, Hitchcock \& Muhlick 14001 (MO); Washington Co., Tamarack, 4436'N, 116 ${ }^{\circ} 50^{\prime} \mathrm{W}, 8-\mathrm{VIII}-1911$, Clark 204 (MO). MonTANA: Beaverhead Co., E of summit of Sheep Mtn T14S, R1W, S33, 45 $28^{\prime} \mathrm{N}, 113^{\circ} 45^{\prime} \mathrm{W}, 24-\mathrm{VIII}-1979$, Lowry 3032 (MO); Big Horn Co., Busby, 45 $32^{\prime} \mathrm{N}$, $106^{\circ} 57^{\prime} \mathrm{W},-\mathrm{VI}-$, Grinnell s.n. (MO); Fergus Co., $5 \mathrm{mi}$ from mouth of Half Moon Canyon, Big Snowy Mts, $46^{\circ} 49^{\prime} \mathrm{N}, 109^{\circ} 16^{\prime} \mathrm{W}, 5-\mathrm{VII}-1945$, Hitchcock \& Muhlick 11961 (MO); Flathead Co., $1 \mathrm{mi}$ S of Columbia Falls, $48^{\circ} 22^{\prime} \mathrm{N}, 114^{\circ} 10^{\prime} \mathrm{W}, 2-\mathrm{VII}-1942$, Rogers \& Rogers 1029 (MO); Gallatin Co., Bozeman, $45^{\circ} 41^{\prime} \mathrm{N}, 111^{\circ} 1^{\prime} \mathrm{W}, 20$ VII-1905, Blankinship 102 (MO); Granite Co., between Philipsburg and Rock Creek, 46 $19^{\circ}$ 'N, $113^{\circ} 18^{\prime} \mathrm{W}, 19-\mathrm{VII}-$ 1946, Hitchcock \& Muhlick 14725 (MO); Lake Co., N side of Mission Reservoir, Mission Range, $47^{\circ} 18^{\prime} \mathrm{N}, 114^{\circ} 1^{\prime} \mathrm{W}$, 29-VI-1962, Cottam 17154 (MO); Lewis \& Clark Co., McDonald Pass, W of Helena, 47 $0^{\prime} \mathrm{N}, 112^{\circ} 31^{\prime} \mathrm{W}, 22-$ VIII-1976, Kral $58796 b$ (MO); Lewis and Clark Co., Helena, $46^{\circ} 36^{\prime} \mathrm{N}, 112^{\circ} 0^{\prime} \mathrm{W}, 12-\mathrm{IX}-1884$, Seymour s.n. (MO); Madison Co., $4 \mathrm{mi} \mathrm{W}$ of Pony, 45 $39^{\prime} \mathrm{N}, 11^{\circ} 53^{\prime} \mathrm{W}, 15-$ VII-1968, Cox \& al. 746 (MO); Missoula Co., Sherman Gulch, 46 $54^{\prime} \mathrm{N}, 114^{\circ} 11^{\prime} \mathrm{W}, 19-\mathrm{VI}-1940$, Chapman 19 (MO); Silver Bow Co., near Butte, $45^{\circ} 59^{\prime} \mathrm{N}, 112^{\circ} 32^{\prime} \mathrm{W}$, -1893, Moore s.n. (MO); Stillwater Co., at Independence, head of Boulder Creek, Absaroka National Forest, $45^{\circ} 31^{\prime} \mathrm{N}, 109^{\circ} 27^{\prime} \mathrm{W}, 7-\mathrm{VIII}-1945$, Hitchcock \& Muhlick 13351 (MO). Nebraska: Sioux Co., West Hat Creek Canyon, $42^{\circ} 45^{\prime} \mathrm{N}, 103^{\circ} 46^{\prime} \mathrm{W}, 25-\mathrm{VII}-1940$, Tolstead s. $n$. (NEB). Nevada: Camp Kalleck, ${ }^{\circ}{ }^{\prime} \mathrm{N},{ }^{\circ}$ 'W, 26-VIII-1876, Palmer 9973 (MO); Elko Co., $10 \mathrm{mi} \mathrm{W}$ of Mountain City on Chicken Creek, 41 ${ }^{\circ}$ 'N, $116^{\circ} 6^{\prime} \mathrm{W}, 15$-VII-1937, Nichols \& Lund 402 (MO); Elko Co., Gold Creek, $41^{\circ} 42^{\prime} \mathrm{N}, 115^{\circ} 42^{\prime} \mathrm{W}, 27-\mathrm{VII}-1912$, Nelson \& Macbride 2129 (MO); Elko Co., Lamoille Canyon, Ruby mts, $41^{\circ} 4 \mathrm{l}^{\prime} \mathrm{N}, 115^{\circ} 28^{\prime} \mathrm{W}, 28$-VII-1940, Munz 16185 (UC). OREGon: Baker Co., Wallowa Mts., Pine Creek, $44^{\circ} 28^{\prime} \mathrm{N}$, $117^{\circ} 37^{\prime}$ W, 30-VI-1935, Jones 7197 (MO); Crook Co., Ochoco National Forest, Ochoco Mountains, along Hwy

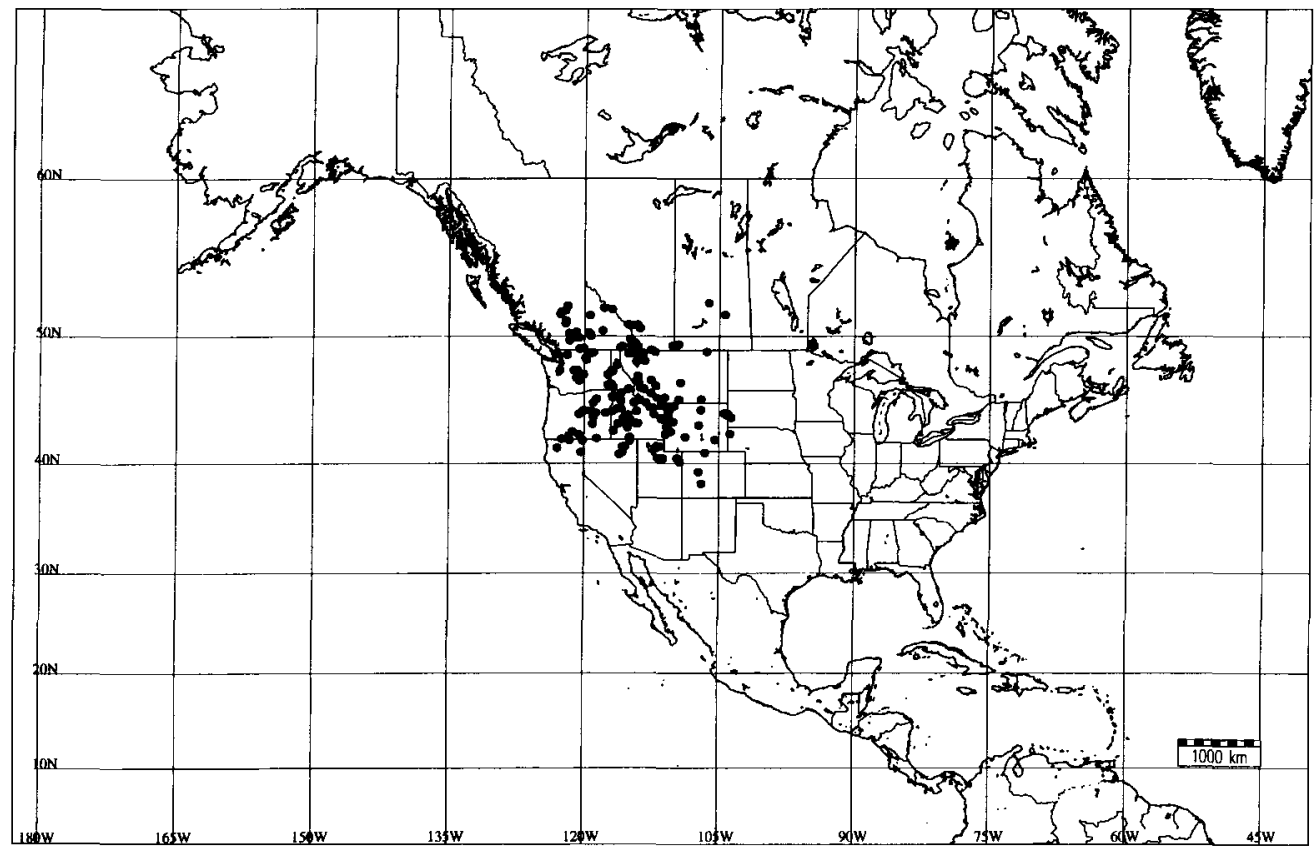

Fig. 33.-Distribution of Geranium viscosissimum. 
26, $4 \mathrm{mi}$ NE of intersection with Forest Service Road 22, $44^{\circ} 22^{\prime} \mathrm{N}, 120^{\circ} 32^{\prime} \mathrm{W}, 18-\mathrm{VII}-1993$, Merello \& al. 800 (MO); Grant Co., Fox Valley, 4436' N, $119^{\circ} 12^{\prime} \mathrm{W}, 15-\mathrm{VI}-$ 1935, Sipe s.n. (OSC); Harney Co., Spring Creek nrth of Burns, $43^{\circ} 35^{\prime} \mathrm{N}, 119^{\circ} 3^{\prime} \mathrm{W}, 16$-VI-1927, Leach 975 (OSC); Harney Co., Steins mts., Wildhorse Creek, $42^{\circ} 24^{\prime} \mathrm{N}, 118^{\circ} 36^{\prime} \mathrm{W}, 9-\mathrm{VI}-1927$, Henderson 6683 (OSC); Jackson Co., Dead Indian Road, 42 $20^{\prime} \mathrm{N}, 122^{\circ} 27^{\prime} \mathrm{W}, 7-$ VII-1931, Peck 16713 (OSC); Klamath Co., Swan Lake Valley, $42^{\circ} 15^{\prime} \mathrm{N}, 121^{\circ} 36^{\prime} \mathrm{W}, 20$-VI-1897, Applegate 967 (OSC); Lake Co., S edge Fremont National Forest, $42^{\circ} 56^{\prime} \mathrm{N}, 121^{\circ} 18^{\prime} \mathrm{W}, 23-\mathrm{VI}-1928$, Constance 9581 (OSC); Umatilla Co., Battle mt., $45^{\circ} 16^{\prime} \mathrm{N}, 118^{\circ} 58^{\prime} \mathrm{W}, 28$-VI1951, Peck 26347a (OSC); Wallowa Co., Buckhorn Spr., $45^{\circ} 45^{\prime} \mathrm{N}, 116^{\circ} 50^{\prime} \mathrm{W}, 29-\mathrm{VI}-1934$, Peck 18320 (OSC);

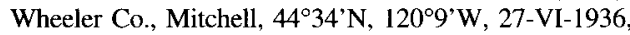
Andrews 662 (OSC). SouTH DAKOTA: Lawrence Co., Iron Creek Lake, Black Hills, $44^{\circ} 22^{\prime} \mathrm{N}, 103^{\circ} 58^{\prime} \mathrm{W}, 26-\mathrm{VI}-$ 1961, Comte 4048 (MO); Pennington Co., Castle Creek, near Deerfield, $44^{\circ} 5^{\prime} \mathrm{N}, 103^{\circ} 37^{\prime} \mathrm{W}, 22-\mathrm{VI}-1929$, Palmer 37480 (MO). UTAH: Cache Co., near Wellsville, $41^{\circ} 38^{\prime} \mathrm{N}$, $111^{\circ} 55^{\prime} \mathrm{W}, 3$-VII-1932, Moore 1042 (MO); Cache Valley, $41^{\circ} 45^{\prime} \mathrm{N}, 111^{\circ} 49^{\prime} \mathrm{W}, 17-\mathrm{VI}-1898$, Mulford 137 (MO); Ephraim Plateau, 404' N, $11^{\circ} 40^{\prime} \mathrm{W}, 3$-VIII-1927, Harris 27706 (MO); Salt Lake Co., Wasatch Range, Red Butte Canyon, $40^{\circ} 45^{\prime} \mathrm{N}, 111^{\circ} 49^{\prime} \mathrm{W}, 4$-VIII-1968, Arnow 2351 (MO); Summit Co., Bear River, 40 $43^{\prime} \mathrm{N}, 111^{\circ} 12^{\prime} \mathrm{W}, 30$ VI-1926, Payson \& Payson 4829 (MO); Uintah Mts., Brush Creek Canon, 40²4'N, 109'20'W, 7-VII-1902, Goodding I29I (MO). WASHINGTON: Chelan Co., Leavenworth, $47^{\circ} 45^{\prime} \mathrm{N}, 120^{\circ} 45^{\prime} \mathrm{W}, 18-\mathrm{V}-1928$, St. John \& al. 9495 (MO); Douglas Co., on Badger Mt., 47 $26^{\prime} \mathrm{N}$, $120^{\circ} 4^{\prime} \mathrm{W}, 1-\mathrm{VI}-1940$, Thompson 14631 (MO); King Co., Tieton Ranger Station, Snoqualmie National Forest, $48^{\circ} 53^{\prime} \mathrm{N}, 121^{\circ} 53$ 'W, 25-V-1934, Murie 1163 (MO); Kittitas Co., near Virden, $47^{\circ} 12 ’ \mathrm{~N}, 120^{\circ} 42^{\prime} \mathrm{W}, 8$-VI-1935, Thompson 11607 (MO); Okanogan Co., $1.5 \mathrm{mi}$ E of Havilla, $48^{\circ} 33^{\prime} \mathrm{N}, 19^{\circ} 44^{\prime} \mathrm{W}, 25-\mathrm{VII}-1937$, Dillon 881 (MO); Spokane Co., near Spangle, $47^{\circ} 25^{\prime} \mathrm{N}, 17^{\circ} 22^{\prime} \mathrm{W}, 29-\mathrm{VI}-$ 1916, Suksdorf 8710 (MO); Stevens Co., along Columbia River $3 \mathrm{mi}$ above mouth of Spokane River, $47^{\circ} 40^{\prime} \mathrm{N}$, $116^{\circ} 48^{\prime} \mathrm{W}, 6-\mathrm{V}-1940$, Rogers 396 (MO); Whitman Co., Pullman, $46^{\circ} 43^{\prime} \mathrm{N}, 117^{\circ} 10^{\prime} \mathrm{W}, 26-\mathrm{VI}-1893$, Piper s.n. (MO). Wyoming: Albany Co., Laramie Peak, $42^{\circ} 16^{\prime} \mathrm{N}$, 105²6'W, 13-VII-1900, Nelson 7591 (MO); Big Horn Co., head Middle Fork Powder River, $43^{\circ} 29^{\prime} \mathrm{N}$, 107 14'W, 18-VII-1901, Goodding 286 (MO); Carbon Co., Copperton, 20-VI-1901, Tweedy 4591 (NY); Crook Co., Wagon Canyon, 44 $18^{\prime} \mathrm{N}, 104^{\circ} 7^{\prime} \mathrm{W}, 23-\mathrm{VII}-1996$, Rolfsmeier 12665 (NEB); Fish Creek, Teton Forest Reserve, -VII-1897, Tweedy 494 (NY); Fremont Co., near Atlantic City, $42^{\circ} 29^{\prime}$ N, $108^{\circ} 43^{\prime}$ W, 7-VII-1949, Porter 4991 (MO); Lincoln Co., Afton, $42^{\circ} 43^{\prime} \mathrm{N}, 110^{\circ} 55^{\prime} \mathrm{W}, 5-\mathrm{VII}-$ 1923, Payson \& Armstrong 3372 (MO); Mount Washburn,

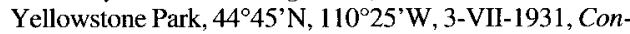
don 5744 (MO); Sheridan Co., above Big Horn, $44^{\circ} 41^{\prime} \mathrm{N}$, 106 $59^{\prime} \mathrm{W}, 25-\mathrm{VI}-1897$, Pammel \& Stanton s.n. (MO); Sublette Co., Horse Creek, $7 \mathrm{mi}$ W of Merna, $42^{\circ} 56^{\prime} \mathrm{N}$, $110^{\circ} 20^{\prime} \mathrm{W}, 17-\mathrm{VII}-1922$, Payson \& Payson 2733 (MO); Sundance, $44^{\circ} 26^{\prime} \mathrm{N}, 104^{\circ} 24^{\prime} \mathrm{W}, 3-\mathrm{VII}-1896$, Nelson 2143 (MO); Teton Co., $3.8 \mathrm{mi} \mathrm{S}$ of Jackson on route 187 , $43^{\circ} 28^{\prime} \mathrm{N}, 110^{\circ} 45^{\prime} \mathrm{W}, 7-\mathrm{VIII}-1979$, Davis 932 (MO).

\section{Geranium subg. Robertium}

Fruit of "carpel-projection-type" with seeds actively discharged by the explosive recoiling of the awn. The whole mericarp, containing the seed, is dispersed, whereas the awn remains with the columella; mericarps without basal callus or prong.

18.Geranium pyrenaicum Burm. f. subsp. pyrenaicum, Spec. Bot. Geran.: 27 (1759) Ind. loc.: "Habitat in Pyrenaeis"

Typus: authentic specimens in $\mathrm{G}$, lectotype not designed (see AEDO \& al. 1998: 615)

Herbs perennial, 15-70(-110) $\mathrm{cm}$ tall. Rootstock 3.5-11.2 mm diam., vertical, not tuberculate, turnip-shaped. Stem erect, usually few per caudex branch, with long eglandular hairs 1-1.4 mm long and short glandular and eglandular hairs $<0.5 \mathrm{~mm}$ long. Basal leaves in a persistent rosette; lamina 2.8-6.2 $\times$ 2.5-7.5 cm, orbicular to reniform in outline, cordate, palmatifid (divided for 0.5-0.6 of its length), pilose, with appressed, eglandular hairs; segments 5-7, obdeltate, 4-8 $\mathrm{mm}$ at the base, (3-)7-10(-12)-lobed at apex (ratio mainsinus length of the middle segment / middlesegment length $=0.15-0.18$ ); cauline leaves opposite; petioles to $25 \mathrm{~cm}$ long, with patent, long eglandular hairs $1-1.5 \mathrm{~mm}$ long, and short glandular and eglandular hairs $<0.5 \mathrm{~mm}$ long; stipules 3-9 $\times 1-3.5 \mathrm{~mm}$, lanceolate, sometimes lobed, with eglandular hairs on

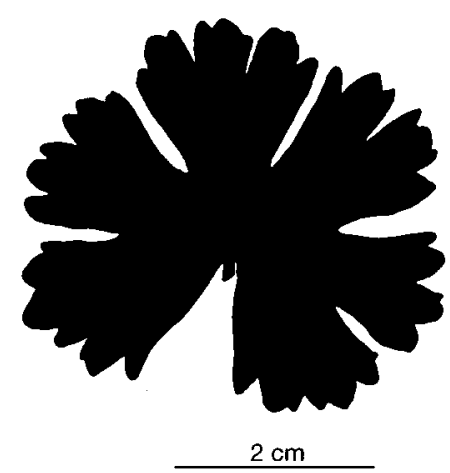

Fig. 34.-Silhouette of lamina of Geranium pyrenaicum [Aedo 3856 (MA)]. 
abaxial surface, glabrous adaxially. Inflorescence a dichasial cyme; cymules 2-flowered, solitary; peduncles $1-3.8 \mathrm{~cm}$ long, with patent, eglandular hairs 1-1.3 mm long and short $(<0.5 \mathrm{~mm})$ glandular and eglandular hairs; bracteoles 2.5-5 × 0.5-0.8 mm, lanceolate, sometimes lobed, with eglandular hairs on abaxial surface and on the margin, glabrous adaxially; pedicels $1-3 \mathrm{~cm}$ long, with short $(<0.5 \mathrm{~mm})$ glandular and eglandular hairs and sometimes with patent, eglandular hairs $0.7-1.3 \mathrm{~mm}$ long; pedicel and peduncle together overtopping the subtending leaf. Sepals $3.5-5 \times 1.6-2.5 \mathrm{~mm}$ (ratio pedicel length $/$ sepal length $=2.2-4.5$ ), smooth, not accrescent, 3-5-nerved, with mucro 0.2-0.3 $\mathrm{mm}$ long, with scarious margins ca. $0.1 \mathrm{~mm}$ wide, with short $(<0.5 \mathrm{~mm}$ ) eglandular and glandular hairs on the abaxial side, glabrous adaxially. Petals 7-11 $\times 5-6.5 \mathrm{~mm}$ (ratio petal length / petal width $=1.4-1.6$ ), erect-patent, emarginate (notch 2-3 mm deep), with short claw, glabrous on both sides, ciliate on the basal margin, bright purple. Stamens 10 , both whorls bearing anthers; filaments $4-5 \mathrm{~mm}$ long, not exserted, lanceolate, pilose on the abaxial side, ciliate on the proximal half, with hairs 0.1-0.2 mm long; anthers 1-1.2 $\times 0.5-0.7$ $\mathrm{mm}$. Nectaries glabrous. Gynoecium 4-4.5 $\mathrm{mm}$ long. Fruit 18-20 mm long, erect; mericarps 2.4-3.1 $\times 1.1-1.4 \mathrm{~mm}$, without a strand of fibres, smooth, with longitudinal rib, without basal beak, without a basal callus, with appressed, eglandular hairs ca. $0.1 \mathrm{~mm}$ long or glabrous, not ciliate at the base, brownish; rostrum $10-15 \mathrm{~mm}$ long, without a narrowed apex, with erect-patent, eglandular and glandular hairs ca. $0.1 \mathrm{~mm}$ long; stigmatic remains 1.5-1.8 $\mathrm{mm}$ long (ratio fruit length / stigmatic remains length $=8.5-10.1$ ), with 5 hairy lobes. Seeds $2.2-2.7 \times 1.2-1.4 \mathrm{~mm}$, finely reticulate, brownish; hilum 1/5-1/6 as long as the perimeter. Cotyledons entire. $2 n=26, n=13$.

Flowering June-July. Roadsides and waste grounds; 0-100 m. Europe, Caucasus, Western Asia \& Northern Africa; introduced from Old World in Southwestern U.S.A., Eastern Canada \& Northeastern U.S.A. Fig. 35.

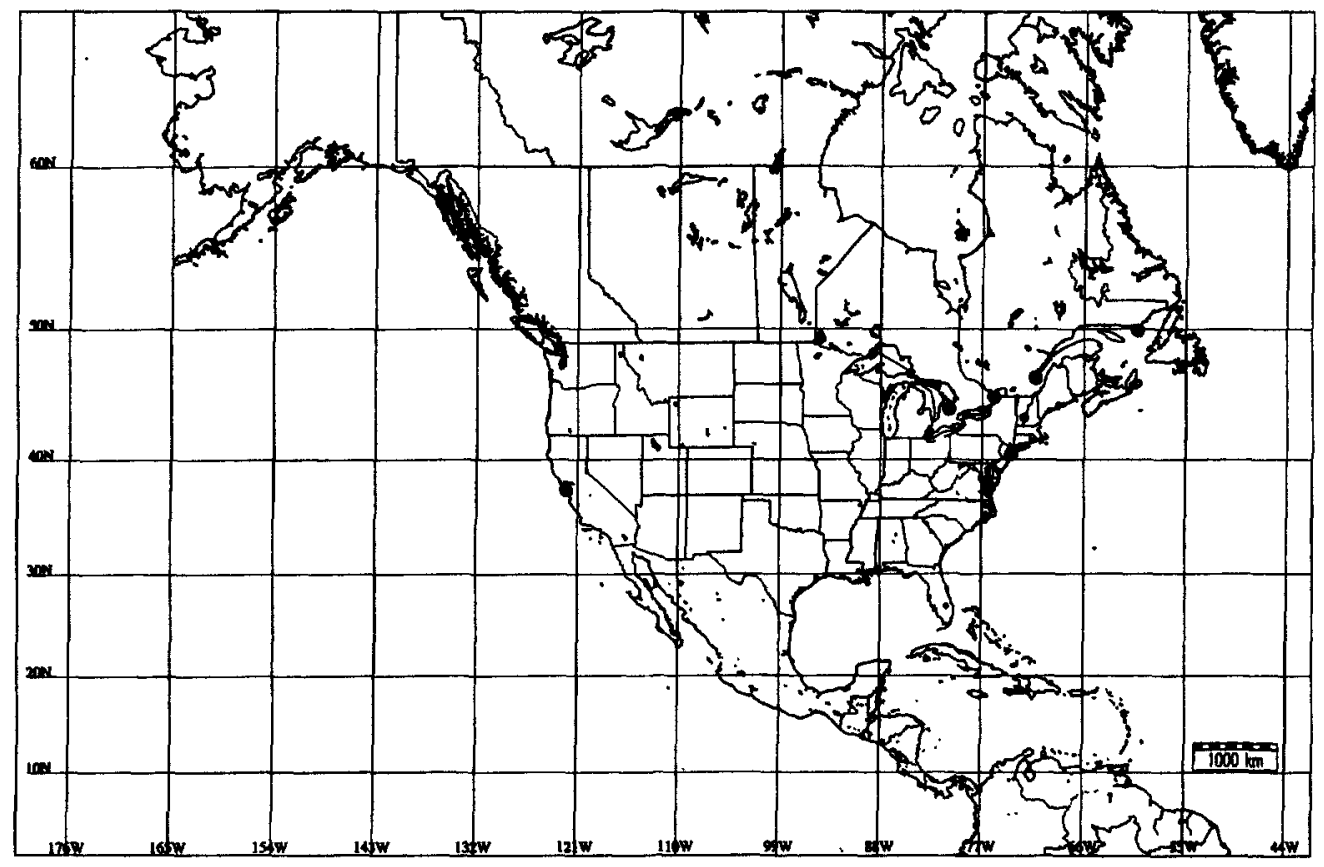

Fig. 35.-Distribution of Geranium pyrenaicum in North America. 
Illustrations. Fig. 34. AEDO \& AL. (1998: 617 fig. 11).

This species is represented in North America by subsp. pyrenaicum, which is also widespread in the Old World. Geranium pyrenaicum subsp. lusitanicum (Samp.) S. Ortiz, restricted to Iberian Peninsula, is easily distinguished from subsp. pyrenaicum by its glabrous mericarps. A discussion on G. pyrenaicum variability can be found in AEDO \& al. (1998: 614).

According to RousseaU (1968: 103) this species is naturalised in Canada around 1930. The specimens supporting the records of $G$. pyrenaicum from Michigan (Voss 1985: 503; KARTESZ 1998) have not been studied.

\section{Representative specimens examined}

Canada. Ontario: Grey Co., W of Beaverdale, $44^{\circ} 24^{\prime} \mathrm{N}, 80^{\circ} 38^{\prime} \mathrm{W}, 20$-VI-1973, Whiting 1666 (CAN). QuÉBEC: Duckpond, Dow's Lake, Central Exp. Farm, Ottawa, $45^{\circ} 23^{\prime} \mathrm{N}, 75^{\circ} 42^{\prime} \mathrm{W}, 8$-VI-1935, Minshall s.n. (DAO); Duck Island, $50^{\circ} 14^{\prime} \mathrm{N}, 60^{\circ} 13^{\prime} \mathrm{W}, 27-\mathrm{VI}-1933$, Linshall s.n. (DAO); Anse-au-Foulon, 28-VI-1945, Cayouette \& Cinq-Mars s.n. (DAO); Sillery, chemin de Foulons, $46^{\circ} 46^{\prime} \mathrm{N}, 71^{\circ} 15^{\prime} \mathrm{W}, 11-\mathrm{VI}-1985$, Ayotte $85-20$ (CAN); Québec, $46^{\circ} 50^{\circ} \mathrm{N}, 71^{\circ} 15^{\prime} \mathrm{W}, 12-\mathrm{VIII}-1934$, Louis-Marie s.n. (MA).

USA. Calurornia: Alameda Co., Berkeley campus, east side of Botany building, $37^{\circ} 52^{\prime} \mathrm{N}, 122^{\circ} 16^{\prime} \mathrm{W}, 15-$ VII-1914, Jepson s.n. (JEPS). NEW YORK: Bronx Co., New York Botanical Garden, Bronx Park, $40^{\circ} 51^{\prime} \mathrm{N}$, 73'52'W, 20-IX-1940, Gilly 426 (NY). VerMONT: Windsor Co., Woodstock, $43^{\circ} 38^{\prime} \mathrm{N}, 72^{\circ} 30^{\prime} \mathrm{W}, 15-\mathrm{VII}-1962$, Sawyer 205 (VT).

19.Geranium palmatum Cav., Diss. $4: 216$, tab. 84 fig. 2 (1787)

Ind. loc.: "Habitat... Habui floridum mensibus iunio, iulio et augusto. Colitur in R.H.P."

Typus: Colitur in R.H.P., Cavanilles s.n. (lectotype, here designated, MA-475746!)

Herbs perennial, 40-60(-120) $\mathrm{cm}$ tall. Rootstock 6-11 mm diam., vertical, not tuberculate, not turnip-shaped. Stem erect, few per caudex branch, glabrous. Basal leaves in a persistent rosette; lamina 9.3$15(-30) \times 11-16(-35) \mathrm{cm}$, polygonal in outline, cordate, palmatisect, pilose, with \pm ap- pressed, glandular hairs on both sides, and eglandular hairs on adaxial side; segments 5 , rhombic (middle segment with a petiolule $0.2-0.5$ as long as its lamina), $1 \mathrm{~mm}$ at the base, 50-160-lobed in distal half (ratio main-sinus length of the middle segment / middle-segment length $=0.40-0.46$ ); cauline leaves opposite; petioles to $38 \mathrm{~cm}$ long, with scattered patent, glandular hairs 0.4-1.1 mm long; stipules 40-72 $\times 25-55$ $\mathrm{mm}$, broadly lanceolate, with glandular and eglandular hairs on both surface and on the margin. Inflorescence a dichasial cyme; cymules 2-flowered, solitary; peduncles 3.5$10 \mathrm{~cm}$ long, with patent, glandular hairs $0.4-$ $2 \mathrm{~mm}$ long and retrorse, not appressed, eglandular hairs 0.2-0.4 mm long; bracteoles $1.5-3 \times 0.8 \mathrm{~mm}$, lanceolate, with glandular hairs on abaxial surface and on the margin, glabrous adaxially; pedicels $1-3.3 \mathrm{~cm}$ long, with patent, glandular hairs 0.4-2 $\mathrm{mm}$ long and retrorse, not appressed, eglandular hairs 0.2-0.4 mm long; pedicel and peduncle together overtopping the subtending leaf. Sepals 6.5-9 $\times 2.8-4.5 \mathrm{~mm}$ (ratio pedicel length $/$ sepal length $=1.1-5$ ), smooth, not accrescent, 3-nerved, with mucro $2.2-3 \mathrm{~mm}$ long, with scarious margins $0.2-0.5 \mathrm{~mm}$ wide, with \pm patent glandular hairs 0.4-2.5 $\mathrm{mm}$ long on the abaxial side, glabrous adaxially. Petals $19-21 \times 7-11 \mathrm{~mm}$ (ratio petal length / petal width $=1.9-2.8)$, \pm patent, entire, with claw 3-5.5 mm long (bicarinated), glabrous, purplish. Stamens 10, both whorls bearing anthers; filaments $13-15 \mathrm{~mm}$ long, exserted, lanceolate, glabrous; anthers 1.8-2 $\times 0.6 \mathrm{~mm}$. Nectaries glabrous. Gynoecium 10-12 mm long. Fruit 27-33 mm long, erect when immature; mericarps 3.6-4 × 1.4-1.5 $\mathrm{mm}$, without a strand of fibres, reticulate (the ridges closer together distally and forming 1-2 annular keels at the apex), without longitudinal rib, without basal beak, without a basal callus, glabrous, brownish; rostrum 21-24 mm long, with a narrowed apex 7-15 $\mathrm{mm}$ long, glabrous in the proximal half, with patent, glandular and eglandular hairs 0.1-0.3 mm long on the narrowed apex; stigmatic remains $2.5-3 \mathrm{~mm}$ long (ratio fruit 


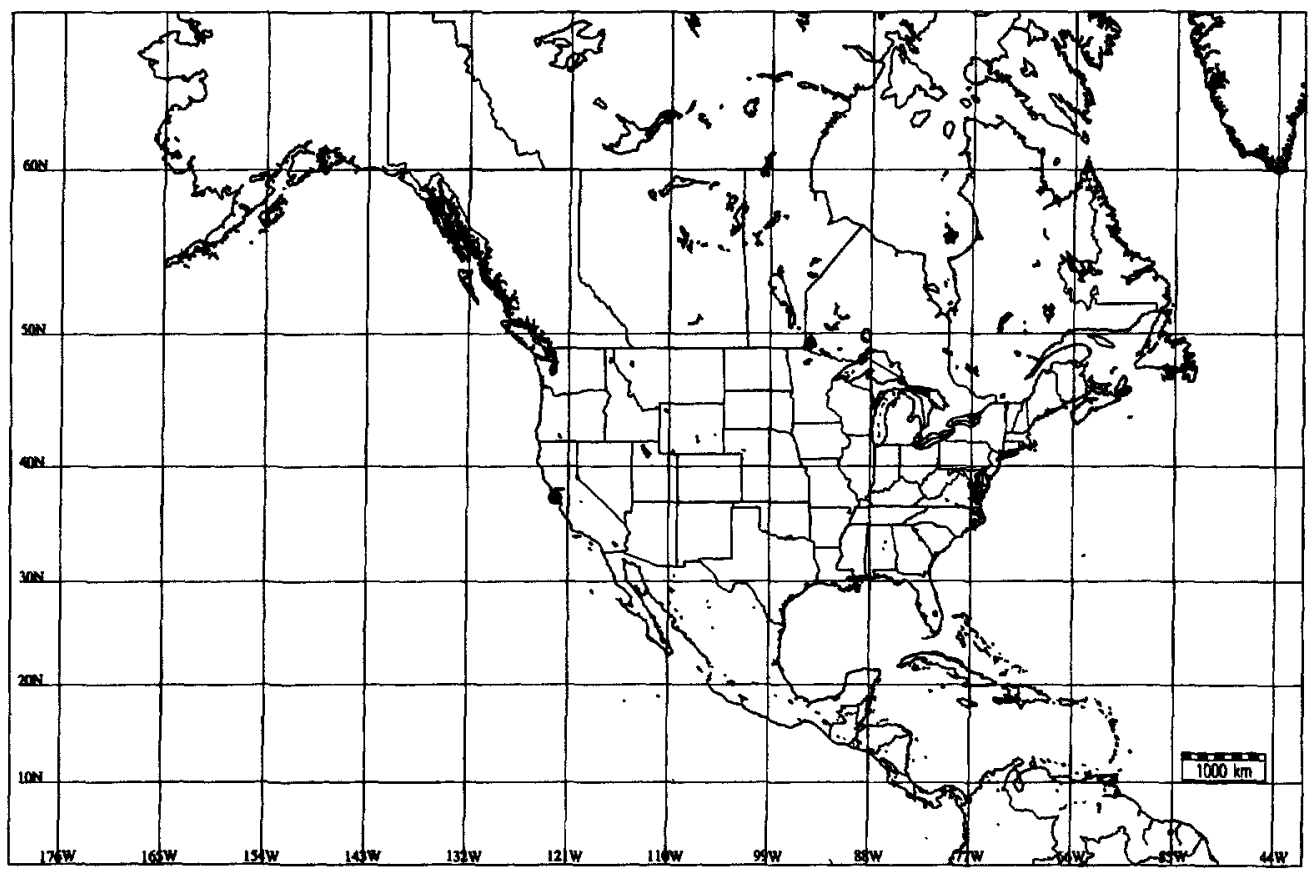

Fig. 36.-Distribution of Geranium palmatum in North America.

length / stigmatic remains length $=9-13.2$ ), with 5 glabrous lobes. Seeds 2.4-2.5 × 1.1$1.3 \mathrm{~mm}$, finely reticulate, brownish; hilum $1 / 6$ as long as the perimeter. Cotyledons entire. $2 n=68, n=34$.

Flowering June. Roadside; 0-100 m. Macaronesia; introduced from Old World in Southwestern U.S.A.. Fig. 36.

Illustrations. YEO (1973: 326 fig. 11; 1992a: 162 fig. 9.88).

Nomenclature and variability of this species is exhaustively analysed by YEO (1973: 326). Geranium palmatum is endemic to Madeira. It can be distinguished from other Macaronesian Geranium by its larger stamens (up to $17 \mathrm{~mm}$ ) exerted from the throat of the flower more than half of their length.

\section{Representative specimens examined}

USA. California: San Francisco Co., Golden Gate Park, road above de Laveaga Dell, $37^{\circ} 46^{\prime} \mathrm{N}, 122^{\circ} 28^{\prime} \mathrm{W}$, 14-VI-1990. Mc Clintock s.n. (JEPS).

\section{HYBRYDS}

Geranium ibericum $\times$ G. platypetalum Geranium $\times$ magnificum Hyl.

KARTESZ (1998) indicates the presence in Connecticut, Maryland, Massachusetts, New Jersey, New York, Colorado, of G. ibericum Cav. as "confirmed". ScoGGAN (1978: 1045) recorded this species in Newfoundland, as an occasional garden-escape, probably not established. I have examined three flowering specimens identified as " $G$. ibericum" from North America: Newfoundland, Beach Cove, 1928, Ayre 605 (GH); Maryland, Bar Harbor, 2-VI-1910, unknown collector (GH); and Colorado, Boulder Creek, 16-VI-1993, EPOB 4520 (COLO). According to YEO's (1992a) key and description, they seem to be Geranium $\times$ magnificum, which should be confirmed when fruiting material is available. This hybrid is best distinguished by its failure to ripen seeds (YEO, 1992a: 139). The three mentioned specimens have glandular hairs on the 
cymules and sepals, while G. ibericum has only eglandular hairs.

\section{ACKNOWLEDGEMENTS}

The author thanks S. Cafferty (BM) for his help with Linnaean typifycations [the choices of $G$. pratense and $G$. sanguineum types have been made by B. Jonsell (SBT) and C. Jarvis (BM)]; G. Baillargeon for his help on Canadian localities; N. Lederer and T. Ranker (COLO) who tried to get fruits of G. ibericum; F. Ganders (UBC), D. Fabijan (ALTA), and J. Kuijt (UVIC) for their help with the distribution of $G$. oreganum; A. Rhoads and T. Block (MOAR) for their help with the distribution of G. versicolor; S. Welsh (BRY) for his help with the distribution of $G$. sanguineum; $C$. Anderson (MICH), R. Gauthier (QFA), R. K. Rabeler (MICH), and C. Ulloa (MO) for their bibliographic assistance; R. Noya for correcting the first English version of the manuscript; $M$. Velayos and an anonymous reviewer for their critical review of the manuscript; and S. Castroviejo for his uncompromised support. I am also grateful to the curators of the cited herbaria (see material and methods) for their kind assistance during my visits and for specimen loans. This work was partly financed by the Spanish Dirección General de Investigación Científica y Técnica (DGICYT) through the research projects PB96-0849, AMB1999-1290-E, and REN2000-2093-E/GLO.

\section{REFERENCES}

AEDo, C., J.J. Aldasoro \& C. Navarro (1998). Taxonomic revision of Geranium L., sections Divaricata Rouy and Batrachioidea W. D. J. Koch (Geraniaceae). Ann. Missouri Bot. Gard. 85: 594-630.

AEDO, C. (2000). The genus Geranium L. (Geraniaceae) in North America. Anales Jard. Bot. Madrid 58(1): 39-82.

BRUGGen, Th. van (1985). The Vascular Plants of South Dakota ed. 2. Ames, Iowa State Univ. Press.

CAROLIN, R.C. (1965). The genus Geranium L. in the South Western Pacific area. Proc. Linn. Soc. New S. Wales 89: 326-361; plates VI-VII.

CAVANLles, A.J. (1787). Monadelphiae classis dissertationes decem. Quarta dissertatio botanica. Paris.

Charkevicz, S.S. (1988). Plantae Vasculares Orientis Extremi Sovietici. Leningrad. [in Russian]

CORREL, D.S. \& M.C. JohNSTON (1979). Manual of the Vascular Plants of Texas. Univ. Texas, Dallas.

Douglas, G.W., G.B. StRaley \& D. MEIDINGeR (1989). The Vascular Plants of British Columbia. BC Ministry of Forest.
HaNKS, L.T. \& J.K. SMall (1907). Geranium L. In: L.T. Britton \& J.K. Small, North American Flora 25: 3-21. New York.

HARDEN, G.J. (1992). Flora of New South Wales, vol. 3. New South Wales Univ. Press, Kensington.

JaCQUIN, N.J. (1770-71). Hortus Botanicus Vindoboniensis... vol. 1. Vindobonae.

JAMES, E. (1823a). Account of an expedition from Pittsburgh to the Rocky mountains... American ed. Philadelphia.

JAMES, E. (1823b). Account of an expedition from Pittsburgh to the Rocky mountains... English ed. London.

JARVIS, C.E., F.R. BarRiE, D.M. All.an \& J.L. ReVEaL (1993). A List of Linnaean Generic Names and their Types. Regnum Veg. 127: 1-100.

JEPSON, W.L. (1925). A manual of the flowerings plants of California. Berkeley.

JONES, G.N. \& F.F. JONES (1943). A revision of the perennial species of Geranium of the United States and Canada. Rhodora 45: 5-26; 32-53.

KaRTESZ, J.T. (ed.) (1998). A Synonymized Checklist of the Vascular Flora of the United States, Puerto Rico, and the Virgin Islands. [http://www.csdl.tamu.edu/ flora/b98/check98.htm].

KNUTH, R. (1912). Geranium L. In: A. Engler (ed.), Das Pflanzenreich. IV.129 (Heft 53): 43-221, 575-583. Leizpig.

MaRTin, W.C. \& Hutchins, C.R. (1980). A Flora of New Mexico. J. Cramer, Vaduz.

MoORE, H.E. (1943). A revision of the genus Geranium in Mexico and Central America. Contr. Gray Herb. 146: 1-108; 5 "Plate".

Munz, P.A. (1959). A California flora. Berkeley, Los Angeles \& London.

PACKER. J.G. (1983). Flora of Alberta. Toronto. University of Toronto Press.

PORTER, D.M. (1972). Geranium potentilloides in Califomia. Madroño 21: 449.

RHOADS. A. F. \& KLEIN, W. MC. (1993). The vascularflora of Pennsylvania and Atlas. American Philosophical Society, Philadelphia.

Ross-CraIG, S. (1952). Drawings of British Plants, Part VI. G. Bell \& Sons. London.

RousSEAU. C. (1968). Histoire, habitat et distribution de 220 plantes introduites au Québec. Naturaliste Canad. 95: 49-169.

SCOGGAN, H.J. (1978). The Flora of Canada. Ottawa.

SWEET, R. (1826-28). Geraniaceae. The natural order of Gerania... London.

TAYLOR. M.S. (1993). Geranium. In: J.C. Hickman (ed.). The Jepson Manual. Univ. California Press, Berkeley, Los Angeles \& London.

ToKARSKI, M. (1972). Morphological and taxonomical analysis of fruits and seeds of the European and Caucasian species of the genus Geranium L. Monogr. Bot. 36: 5-115.

Voss. G.E. (1985). Michigan Flora. Cranbrook Institute of Science \& Univ. Michigan Herbarium.

WeLSH, S.L., N.D. ATWOOD, S. GoODRICH \& L.C. HIGoINS (1993). A Utah Flora, ed. 2. Brigham Young Univ., Provo. Utah. 
YEO, P. (1992a). Hardy Geraniums. London, B. T. Batsford.

Yeo, P.F. (1992b). A revision of Geranium L. in SouthWest China. Edinburgh J. Bot. 49: 123-211.

YEO, P.F. (1973). The biology and systematics of Geranium, sections Anemonifolia Knuth and Ruberta Dum. Bot. J. Linn. Soc. 67: 285-346.

\section{INDEX TO SCIENTIFIC NAMES}

(Accepted names are in roman type; the main entry for each is in boldface. Synonyms are in italics)

\section{Geranium L.}

subg. Geranium, 6

subg. Robertium, 59

sect. Caespitosa, 38

albidum Rydb. ex Hanks \& Small, 52

albiflorum Hook., 49

var. incisum Nutt. ex Torr. \& A. Gray, 14

attenuilobum G.N. Jones \& F.L. Jones, 55, 57

atropurpureum A. Heller, 34

var. cowenii (Rydb.) Dorn, 35

var. furcatum (Hanks) Kearney \& Peebles, 35

caespitosum E. James, 34, 38, 40, 45, 48, 52 subsp. atropurpureum (A. Heller) W.A. Weber, 34

var. eremophilum (Wooton \& Standl.) W.C. Martin \& C.R. Hutchins, 35

var. fremontii (Torr. ex A. Gray) Dorn, 34

var. gracile Engelm. ex A. Nelson \& J.F. Macbr., 34

var. parryi (Engelm.) W.A. Weber, 34

f. albiflorum Cockerell, 34

californicum G.N. Jones \& F.L. Jones, 37, $38,39,40$

calvescens Briq., 46

canum Rydb., 14

concinnum G.N. Jones \& F.L. Jones, 39, 40 cowenii Rydb., 35

dissectum

var. retrorsum (L'Hér. ex DC.) Hook. f., 30

durangensis Rose, 46

elatum (Maxim.) R. Knuth, 6

eremophilum Wooton \& Standl., 35

erianthum DC., 6, 11, 22

var. angustifolium Miyabe \& Tatew., 7

var. elatum Maxim., 6

f. albiflora Kom., 7

f. alpestris Kom., 7

f. communis Kom., 7

f. leucanthum Takeda, 7

eriostemum Fisch. ex DC., 8

var, orientale Maxim., 6

fremontii Torr. ex A. Gray, 34 var. cowenii (Rydb.) H.D. Harr., 35

var. parryi Engelm., 34

furcatum Hanks, 35

geoides Small, 46

glabratum (Hook. f.) Small, 32

gorbizense Aedo \& Muñoz Garm., 6

gracile Engelm., 34

gracilentum Greene, 49

homeanum Turcz., 32

hookerianum Walp., 49

var. incisum (Nutt. ex Torr. \& A. Gray)

Walp., 14

ibericum Cav., 16, 62, 63

incisum (Nutt. ex Torr. \& A. Gray) Howell, 14

intermedium $\mathrm{E}$. James, 34, 38

lentum Wooton \& Standl., 37, 38, 42, 45, 48

leucanthum Small, 39

loloense $\mathrm{H}$. St. John, 50

maculatum L., 10, 11, 16, 38

var. albiflorum Raf., 10

var. album H.M. Myers, 10

var. diphyllum Raf., 10

var. humile Raf., 10

var. macrophyllum Raf., 10

var. parviftora N. Coleman, 10

var. plenum Lauman, 10

var. viride Raf., 10

f. albiflorum Raf. ex House, 10

magnificum Hyl., 62

marginale Rydb. ex Hanks \& Small, 35

nepalense Sweet, 29

nervosum Rydb., 55

oreganum Howell, 11, 14, 16, 38

orientale (Maxim.) Freyn, 6

ornatum A. Nelson ex R. Knuth, 55

palmatum Cav., 61, 62

parryi (Engelm.) A. Heller, 34

pattersoni Rydb., 35

pentagynum Engelm., 34

pilosum Sol., 32

pilosum Sol. ex Willd., 32

var. retrorsum (L'Hér. ex DC.) Jeps., 30

platyanthum Duthie, 8

potentilloides L'Hér. ex DC., 26, 27, 28, 33

pratense L., 20, 22

var. erianthum (DC.) B. Boivin, 6

prostratum Cav., 20

pyrenaicum Burm. f.

subsp. pyrenaicum, 59, 61

subsp. lusitanicum (Samp.) S. Ortiz, 61

retrorsum L'Hér. ex DC., 30, 32

richardsonii Fisch. \& Trautv., 16, 37, 38, 48,

$49,50,52$

var. intermedium Kuntze, 34

sanguineum $L$., 23, 24

sibiricum L., 27, 28 
solanderi Carolin, 32, 33

striatum L., 30

strigosior H. St. John, 55

strigosum Rydb., 55, 57

sylvaticum L., 11, 18, 20

thunbergii Siebold ex Lindl. \& Paxton, 29, 30

toquimense A.H. Holmgren \& N.H. Holmgren, 35

versicolor L., 30

viscicum, 39 viscosissimum Fisch. \& C.A. Mey., 16, 37, $38,40,55,57$

subsp. nervosum (Rydb.) W.A. Weber, 55

var. album Suksd., 55

var. nervosum (Rydb.) C.L. Hitchc., 55

f. album (Suksd.) H.St. John, 55

wislizenii $S$. Watson, 38, 45, 46, 48

Editado por Carlos Lado Aceptado para publicación: 26-VI-200I 